ENVIRONMENTAL RESTORATION PROGRAM

\section{SWSA 6 Interim Corrective Measures Environmental Monitoring: FY 1990 Results}

T. L. Ashwood

B. P. Spalding 
This report has been reproduced directly from the best available copy.

Available to DOE and DOE contractors from the Office of Scientific and Technical Information, P.O. Box 62, Oak Ridge, TN 37831; prices available from 615-576-8401, FTS 626-8401.

Available to the public from the National Technical Information Service, U.S. Department of Commerce, 5285 Port Royal Rd., Springtield, VA 22161. 
Environmental Restoration Division ORNL Environmental Restoration Program

\title{
SWSA 6 Interim Corrective Measures Environmental Monitoring: FY 1990 Results
}

\author{
T. L. Ashwood \\ B. P. Spalding
}

Manuscript Completed: November 1990

Date Issued-July 1991

Prepared by

Environmental Sciences Division

Oak Ridge National Laboratory

ESD Publication 3722

Prepared for

U.S. Department of Energy

Office of Environmental Restoration and Waste Management

under budget and reporting code EW 20

OAK RIDGE NATIONAL LABORATORY

Oak Ridge, Tennessee 37831-6285

managed by

MARTIN MARIETTA ENERGY SYSTEMS, INC.

for the

U.S. DEPARTMENT OF ENERGY

under contract DE-AC05-84OR21400 


\section{Author Affiliations}

T. L. Ashwood and B. P. Spalding are members of the Environmental Sciences Division, Oak Ridge National Laboratory, Martin Marietta Energy Systems, Inc. 


\section{CONTENTS}

Page

FIGURES

TABLES

vii

ACRONYMS

ix

EXECUTIVE SUMMARY

$\mathrm{xi}$

1. INTRODUCTION

1

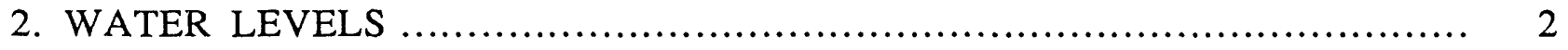

2.1 WELLS OUTSIDE SWSA 6 BURIAL TRENCHES ......................... 3

2.2 WELLS INSIDE SWSA 6 BURIAL TRENCHES .......................... 4

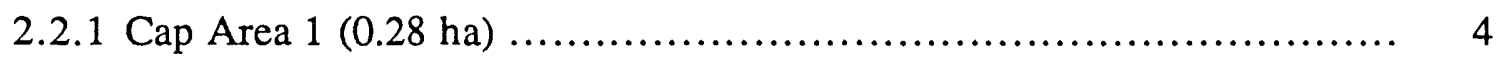

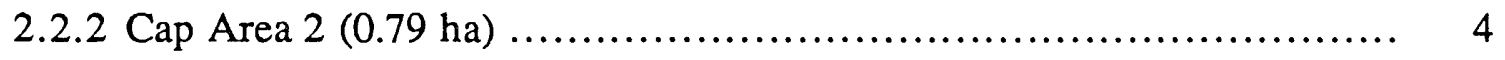

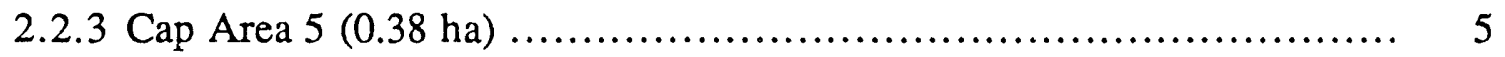

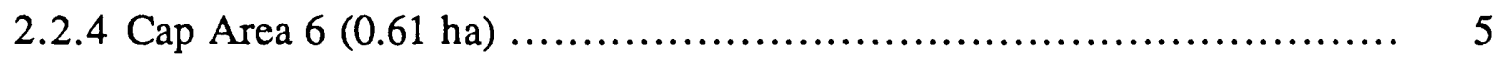

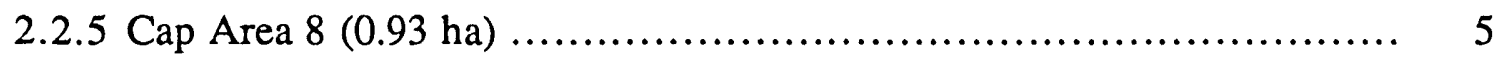

3. PENETRATION RESISTANCE OF SWSA 6 BURIAL TRENCHES ............... 5

4. SAMPLES FROM MONITORING WELLS IN SWSA 6 BURIAL TRENCHES ..... 7

5. CONCLUUSIONS ........................................................ 16

6. ACKNOWLEDGMENTS .................................................. 17

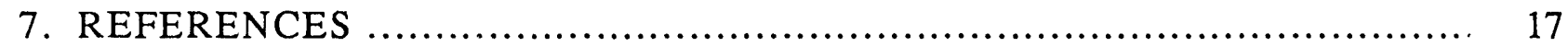

APPENDIX A: Water-level Data From Wells in and Around ICM Caps, Rainfall Data from SWSA 6, and Peak Monthly Elevations of White Oak Lake

APPENDIX B: Procedures for Soil Penetration Testing and Monitoring Well Installation in Radioactive Waste Trenches

APPENDIX C: Penetration Resistance of SWSA 6 Burial Trenches and Control Sites Used for Leachate Monitoring Wells

C-1

APPENDIX D: Chemical Analyses of SWSA 6 Burial Trench Leachates

D-1 


\section{FIGURES}

Figure

1 Solid Waste Storage Area 6 showing interim corrective measures capped areas,

French drain, monitoring wells outside burial trenches, and major low-level waste disposal areas

Hydrograph and associated hyetograph for ICM monitoring well 276

Hydrograph and associated hyetograph for ICM monitoring well 636

Hydrograph and associated hyetograph for ICM monitoring well 642

Hydrograph and associated hyetograph for ICM monitoring well 654

Hydrograph and associated hyetograph for ICM monitoring well 656

Hydrograph and associated hyetograph for ICM monitoring well 646

Hydrograph and associated hyetograph for ICM monitoring well 318

Hydrograph and associated hyetograph for ICM monitoring well 345

Hydrograph and associated hyetograph for ICM monitoring well 356

Hydrograph and associated hyetograph for ICM monitoring well 645

Hydrograph and associated hyetograph for ICM monitoring well 648

Hydrograph and associated hyetograph for ICM monitoring well 655

Hydrograph and associated hyetograph for ICM monitoring well 640

Hydrograph and associated hyetograph for ICM monitoring well 647

Hydrograph and associated hyetograph for ICM monitoring well 368

Hydrograph and associated hyetograph for ICM monitoring well 347

Burial trench and well locations within cap area 1

Hydrograph and associated hyetograph for cap area 1 well $\mathrm{T} 82$..................... 37

Hydrograph and associated hyetograph for cap area 1 well T444

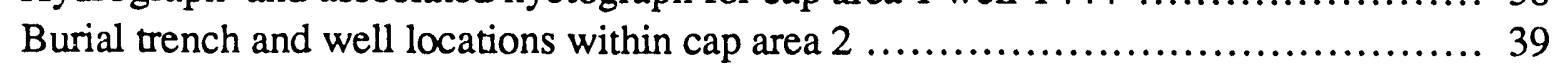

Hydrograph and associated hyetograph for cap area 2 well T69

Hydrograph and associated hyetograph for cap area 2 well T363

Burial trench and well locations within cap area 5

Hydrograph and associated hyetograph for cap area 5 well T85 .................... 44

Hydrograph and associated hyetograph for cap area 5 well T92-1

Hydrograph and associated hyetograph for cap area 5 well T92-2 ................ 46

Hydrograph and associated hyetograph for cap area 5 well T105

Hydrograph and associated hyetograph for cap area 5 well T110

Hydrograph and associated hyetograph for cap area 5 well T112

Hydrograph and associated hyetograph for cap area 5 well T308

Hydrograph and associated hyetograph for cap area 5 well T318

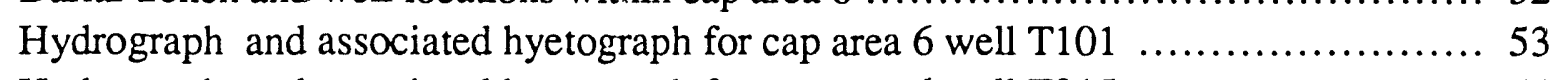




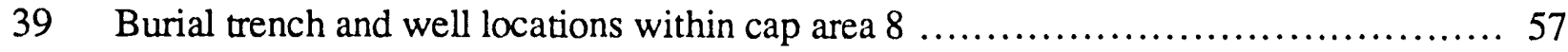

40 Hydrograph and associated hyetograph for cap area 8 well T.44 .................... 58

41 Hydrograph and associated hyetograph for cap area 8 well $\mathrm{T} 60$.....................5 59

42 Hydrograph and associated hyetograph for cap area 8 well T63 ....................6 60

43 Hydrograph and associated hyetograph for cap area 8 well T180 .................6 61

44 Hydrograph and associated hyetograph for cap area 8 well T225 .................6.62

45 Hydrograph and associated hyetograph for cap area 8 well T237 ..................6 63

46 Hydrograph and associated hyetograph for cap area 8 well T352 ...................6 64

47 Hydrograph and associated hyetograph for cap area 8 well T367 ..................6 65

48 Hydrograph and associated hyetograph for cap area 8 well T453 ..................66 66

49 Average penetration resistance of SWSA 6 burial trenches compared with the surrounding soil formation ..................................................... 67

50 Cumulative penetration resistance of SWSA 6 burial trenches compared with the

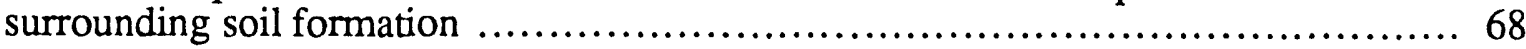

51 Ratio of penetration resistance of SWSA 6 burial trenches to surrounding soil formation as a function of depth 


\section{TABLES}

Table $\quad \underline{\text { Page }}$

1 Summary of chemical analyses of SWSA 6 burial trench leachate samples $\ldots \ldots \ldots \ldots \ldots$

2 Water solubilities of organic compounds analyzed in SWSA 6 burial trench leachate samples

A.1 Depth to water and water-level elevations for ICM monitoring wells outside of burial trenches in SWSA 6

A.2 Locations, elevations, and depth-to-water measurements for intratrench wells in SWSA 6 ICM capped areas

A.3 Daily rainfall in SWSA 6 from October 1988 through September 1990

A.4 Peak monthly elevations of White Oak Lake from October 1988 through September 1990

C. 1 Penetration resistance ( 1 to $3 \mathrm{ft}$ ) of SWSA 6 burial trenches and control sites used for leachate monitoring wells

C.2 Penetration resistance ( 4 to $9 \mathrm{ft}$ ) of SWSA 6 burial trenches and control sites used for leachate monitoring wells

C.3 Penetration resistance ( 10 to $15 \mathrm{ft}$ ) of SWSA 6 burial trenches and control sites used for leachate monitoring wells

D. 1 Chemical analyses of SWSA 6 burial trench leachates ....

D.2 Chemical analyses of SWSA 6 burial trench leachates D-6

D.3

Chemical analyses of SWSA 6 burial trench leachates

D.4 Chemical analyses of SWSA 6 burial trench leachates

D.5 Chemical analyses of SWSA 6 burial trench leachates

D.6 Chemical analyses of SWSA 6 burial trench leachates

Chemical analyses of SWSA 6 burial trench leachates

Chemical analyses of SWSA 6 burial trench leachates

D.9 Chemical analyses of SWSA 6 burial trench leachates

D.10 Chemical analyses of SWSA 6 burial trench leachates D-38 


\section{ACRONYMS}

$\begin{array}{ll}\text { EPA } & \text { Environmental Protection Agency } \\ \text { ESD } & \text { Environmental Sciences Division } \\ \text { HDPE } & \text { High-density polyethylene } \\ \text { ICM } & \text { Interim corrective measure } \\ \text { ICP } & \text { Inductively coupled plasma (spectrometry) } \\ \text { LLW } & \text { Low-level waste } \\ \text { ORNL } & \text { Oak Ridge National Laboratory } \\ \text { PVC } & \text { Polyvinylchloride } \\ \text { RCRA } & \text { Resource Conservation and Recovery Act } \\ \text { SWSA } & \text { Solid Waste Storage Act } \\ \text { TARA } & \text { Test Area for Remedial Actions } \\ \text { TOC } & \text { Total organic carbon }\end{array}$




\section{EXECUTIVE SUMMARY}

This report presents the results and conclusions from a multifaceted monitoring effort associated with the high-density polyethylene caps installed in Solid Waste Storage Area (SWSA) 6 as an interim corrective measure (ICM). The caps were installed between November 1988 and June 1989 to meet Resource Conservation and Recovery Act (RCRA) requirements for closure of those areas of SWSA 6 that had received RCRA-regulated wastes after November 1980. Three separate activities were undertaken to evaluate the performance of the caps: (1) wells were installed in trenches to be covered by the caps, and water levels in these intratrench wells were monitored periodically; (2) samples were taken of the leachate in the intratrench wells and were analyzed for a broad range of radiological and chemical contaminants; and (3) water levels in wells outside the trenches were monitored periodically. With the exception of the trench leachate sampling, each of these activities spanned the preconstruction, construction, and postconstruction periods. Findings of this study have important implications for the ongoing remedial investigation in SWSA 6 and for the design of other ICMs.

The intratrench wells were installed by driving wellpoints into the backfill material in the trench with a 140-1b hammer raised 30 in. This technique provides a standard measure of penetration resistance that can be related to stability of the backfill material. Our results indicate that the stability of the trenches is only about $35 \%$ that of the surrounding soil formation. Thus, significant differential land surface subsidence can be expected for unstabilized burial trenches in SWSA 6 . The capacity of such unstabilized trenches to support infiltration barriers (e.g., composite caps) over the long term must be carefully evaluated.

Water-level measurements from intratrench wells indicate that the ICM caps have been effective in eliminating perched water from the trenches in only two areas (caps 2 and 8 ). Water in burial trenches primarily occurs as a perched water table when shallow storm flow enters the trench faster than it can flow out. Thus, caps are only successful in reducing the perched water table where the caps cover a significant portion of the recharge zone for such shallow storm flow. In other areas (caps 1,5, and 6), the covered burial trenches apparently continue to receive storm flow from upslope recharge areas. No wells were available in trenches beneath caps 3,4 , and 7 .

An important observation from the intratrench wells under cap 8 is the apparent hydrological connection between some trenches and White Oak Lake. Water levels in two trenches whose bottoms were well below the 100-year flood elevation followed the fluctuations in White Oak Lake elevations directly-even though cap 8 appears to be effective in eliminating storm flow from the trenches.

Water levels in the shallow aquifer beneath SWSA 6 were largely unaffected by installation of the caps. This lack of response is probably because the caps do not cover the primary recharge zone for the aquifer near the upper end of SWSA 6 and because the temporary perched water table that occurs in the trenches after a storm slowly percolates from the trenches and recharges the aquifer.

Previously, only limited data have been available on the chemical quality of trench leachates in SWSA 6. Although these leachates represent a direct measure of the source term for contamination from the burial trenches, sampling of the leachates was not part of the remedial investigation in SWSA 6. Our sample results indicate significant concentrations of several volatile organic compounds (approaching their solubility limits) in more than $70 \%$ of the samples. Tritium and elevated gross beta activity were observed in more than $90 \%$ of the samples. Many contaminants were found at concentrations that were much greater than previously reported. 
Our results suggest that use of impermeable caps as interim corrective measures are effective only where they act to eliminate all recharge, including shallow storm flow, in the area of effect. Our results also suggest that final closure of SWSA 6 must address stabilization of the burial trenches and isolation of the trenches from the influence of White Oak Lake. Finally, the trench leachate chemistry suggests that volatile contaminants (organics and tritium) are widespread in the capped burial trenches and that other RCRA-regulated contaminants are present in much greater concentrations than previously recognized. 


\section{INTRODUCTION}

Solid Waste Storage Area (SWSA) 6 is the only operating low-level waste (LLW) disposal site at Oak Ridge National Laboratory (ORNL). Located in Melton Valley southwest of the main plant area, SWSA 6 was opened in 1969 but was not used extensively until burial operations in SWSA 5 were terminated in 1973 (Boegly 1984). The fenced area within SWSA 6 covers 26.7 ha (66 acres), about a third of which is suitable for shallow land burial of LLW (Boegly et al. 1985).

SWSA 6 was closed in May 1986 when it was found that wastes regulated by the Resource Conservation and Recovery Act (RCRA) were being disposed of at the site. Following numerous changes in operations (including cessation of RCRA-regulated waste disposal) the site was reopened in July 1986. Those areas that received hazardous or mixed wastes after November 8, 1980 , were designated as RCRA-regulated units, and closure of those units under 40 CFR 265 Subpart G was required to be initiated before November 1988.

Because closure of the entire SWSA was not feasible, high-density polyethylene (HDPE) covers were installed as an interim corrective measure (ICM) in eight areas believed to have been used for disposal of RCRA-regulated wastes (Fig. 1). The eight caps cover an area of $\sim 4.2$ ha (10.4 acres), or roughly $15 \%$ of the fenced area of SWSA 6. An ICM monitoring program was developed to determine the impact of the ICMs on groundwater, surface water, and sediment transport (Miller and Craig 1988).

Moore $(1988,1989)$ has suggested that less than $10 \%$ of the precipitation infiltrating past the ground surface during a rain event is available to recharge the aquifer. Most infiltrating precipitation is removed by evapotranspiration or flows rapidly toward the surface streams through a shallow ( $<2-\mathrm{m}$ deep) highly permeable storm-flow zone. Trenches, whose backfill is usually much more permeable than the surrounding soil formation, may intercept the storm flow and develop a perched water table because water enters the trench faster than it can percolate out. Impermeable caps act to reduce infiltration and increase runoff. If the caps are successful in reducing infiltration, they would be expected to reduce shallow storm flow and thus lower the perched water table in trenches within their area of effect.

Two separate monitoring programs were instituted in late 1988 to test whether the caps had any effect on the aquifer or the trench water levels. As part of the ICM environmental monitoring program (Miller and Craig 1988), water levels in 25 existing wells outside of the trenches were measured periodically. Results from the first year of this monitoring program suggested that the caps had no measurable impact on water-level elevations in the aquifer under SWSA 6 (Miller et al. 1989). In FY 1990, responsibility for these monitoring activities was assumed by staff of the Environmental Sciences Division (ESD) as part of the Active Sites Environmental Monitoring Program (Ashwood et al. 1990). Results of the ICM environmental monitoring program are presented in Sect. 2.1.

In addition to the ICM environmental monitoring program, staff members of ESD installed drivepoint wells in trenches within the capped areas prior to cap construction (see Sect. 3.0). Water levels in these intratrench wells were monitored manually before, during, and after cap construction, and results are presented in Sect. 2.2. One round of samples was taken from the intratrench wells to assess the potential risk to personnel involved in monitoring water levels and to supplement the limited existing data on quality of trench leachate in SWSA 6 (see Sect. 4.0).

As part of the ICM monitoring program, Miller et al. (1989) collected 41 water samples and 64 sediment samples from surface drainages in SWSA 6. Elevated concentrations of ${ }^{3} \mathrm{H}$ (above the proposed Safe Drinking Water Act Primary Standard of $740 \mathrm{~Bq} / \mathrm{L}$ ) were found in water samples from all areas of the surface streams. Radioactive strontium concentrations in water ranged from below detection to $12 \mathrm{~Bq} / \mathrm{L}$. No elevated levels (above detection limits) of other fission-product 
radionuclides were measured in any water samples, and low gross alpha values $(<1 \mathrm{~Bq} / \mathrm{L})$ suggest that transuranics were not present in the water above detection limits. Sediments contained from 20 to $390 \mathrm{~Bq} / \mathrm{kg}$ of unspecified gross alpha activity, up to $5300 \mathrm{~Bq} / \mathrm{kg}$ of ${ }^{90} \mathrm{Sr}$, up to $940 \mathrm{~Bq} / \mathrm{kg}$ of ${ }^{60} \mathrm{Co}$, and up to $21,000 \mathrm{~Bq} / \mathrm{kg}$ of ${ }^{137} \mathrm{Cs}$. Miller et al. (1989) looked for changes in these variables over time but concliuded that cap construction did not significantly affect concentrations of radionuclides in either the surface water or sediments. Although their grain-size analysis of sediments suggested that fine material was being tran'sported out of SWSA 6 during and immediately following cap construction, the lack of water flow data precluded any quantitative analysis of mass transport of either sediment or radionuclides (Miller et al. 1989).

Without information on water flow and sediment transport from SWSA 6, routine measurements of contaminant concentrations in sediment and water are of little value. For that reason, the limited water and sediment analyses conducted during FY 1990 are not included in this report.

\section{WATER LEVELS}

Sixteen wells outside the burial trenches (Fig. 1) and 25 wells within the trenches constitute the present SWSA 6 ICM groundwater monitoring system. Water levels were measured using an electronic tape in accordance with Energy Systems ESP 302-1 (Kimbrough et al. 1990). Depth-towater measurements were recorded in a field notebook and later transferred to one of two computer programs. For wells outside trenches, depth-to-water data were entered into a data base that also included well construction data, from which water-level elevations were calculated. Data from intratrench wells were entered into a spreadsheet that calculated water-level elevations from information on top-of-casing elevations. During cap construction, casings were occasionally disturbed, and water-level elevations during this period are calculated by measuring the total depth of the well and the distance of water level from the bottom. Both the spreadsheet and the data base are summarized in Appendix A.

Throughout Sects. 2.1 and 2.2, water-level data are presented in well hydrographs that plot water-level elevation against time. For all but the 600-series wells, the abscissa of these hydrographs covers the period from August 1988 through October 1990, though not all wells have data for that entire period. The abscissa of the 600-series wells spans the period from January 1988 through October 1990. Large differences in well elevations precluded the use of a common ordinate scale, except for intratrench wells within the same cap area. In all hydrographs, the ordinate has been chosen to represent the depth of the well. Accordingly, all hydrographs depict the ground surface at the specific well. For the intratrench wells, the hydrographs also depict the bottom of the trench. Each hydrograph also contains a vertical line showing the approximate date (June 1, 1989) when construction was complete on all caps. For various operational reasons, water levels were measured less frequently during FY 1990 than in the preceding period.

To underscore the response, or lack of response, to precipitation in each well, each hydrograph is overlain with a hyetograph that presents the daily rainfall throughout the period of Octoher 1988 through September 1990 (Table A.4, Appendix A). No units are shown in the hyetograph because only the pattern of rainfall is important and inclusion of the units would have made the charts extremely difficult to read. 


\subsection{WELLS OUTSIDE SWSA 6 BURIAL TRENCHES}

Miller et al. (1989) analyzed historical water-level data in 6 wells outside the burial trenches, but within capped areas (wells $347,368,640,647,649$, and 650B), and concluded that no evidence existed to indicate that the ICM caps had significantly reduced groundwater levels in the surrounding area of SWSA 6. Manual water-level monitoring in these wells and other ICM monitoring wells (Table A.1, Appendix A) has continued throughout FY 1990.

Data from 1988 and 1989 are included with the 1990 data in well hydrographs (Figs. 2 through 17) to provide an historical perspective. Well $650 \mathrm{~A}$, the shallow well in a cluster, was always dry throughout the monitoring period, and wells 649 and $650 \mathrm{~B}$ were damaged or blocked during all or part of FY 1990. Hence, their hydrographs are not included in this report. Miller et al. (1989) include hydrographs of wells 649 and 650B for the period preceding FY 1990.

Miller et al. (1989) largely ignored data from the wells outside of capped areas in their analysis. This may have been prompted by the paucity of postcap data on such wells or by the expectation that these wells would be less affected by cap construction than those wells within the cap boundaries. We chose to include data from the outside wells to determine whether the caps were exerting any appreciable control on groundwater hydrology in SWSA 6.

Wells 276, 636, 642, and 654 are relatively distant from any capped areas and are on the opposite side of a surface stream from the closest caps (Fig. 1). Any increased runoff from the caps would be intercepted by the surface drainage and would not be expected to affect the water levels in these wells. Similarly, the surface stream probably serves as a divide between the shallow groundwater on either side. Thus, any reduction in recharge caused by the ICM caps would not be expected to affect water levels in wells $276,636,642$, and 654 . The hydrographs (Figs. 2 through 5) support these predictions, although it must be noted that the monitoring frequency was not great enough to observe short-term changes.

Well 656 is located at the topographic high point in SWSA 6, above the caps and other wells. Well 656 is also one of the deepest $(-37 \mathrm{~m})$ of the ICM monitoring wells. Moore (1988) suggests that less than $10 \%$ of the rainfall in SWSA 6 is available for recharge to the aquifer. Thus, it is not surprising that the hydrograph for well 656 does not show a response to individual rain events (Fig. 6).

Well 646 is above (topographically) cap 3 and is unlikely to be affected by cap 1 because of the surface divide between them. Fostcap water-level elevations are similar to those throughout most of 1988 (Fig. 7). The high water levels during cap construction are also reflected to varying degrees in the hydrographs of each of the wells previously discussed.

Wells $318,345,356,645,648$, and 655 are each topographically (and probably hydrologically) lower than one or more capped areas (Fig. 1). With the exception of well 318 , the hydrographs for these wells show no discernible difference between precap and postcap water-level elevations (Figs. 8 through 13 ).

Well 318 appears to have largely dried out sometime after cap construction (Fig. 8). Those elevations that appear to be at the bottom of the well actually represent times at which there was no water in the well (Table A.1, Appendix A). Although there were dry measurements prior to cap construction, these were interspersed with measurements showing some water in the well. Further monitoring of well 318 coupled with review of the pre-1988 water-level history is needed in order to confirm that the apparent drying of this well is the result of cap construction not just fluctuations within the aquifer under SWSA 6. 
Wells $640,647,368$, and 347 lie within cap areas $1,2,3$, and 5, respectively (Fig. 1). With the exception of well 347 , each of these wells is deeper than $10 \mathrm{~m}$, and they monitor the shallow groundwater aquifer below the burial trenches. Well 347 also monitors the shallow aquifer, which is closer to the surface in the area below cap 5.

Wells 640,647 , and 368 show relatively small fluctuations $(\leq 2 \mathrm{~m})$ in water levels over the entire period of the hydrographs (Figs. 14 through 16). There is no apparent difference in the water-level elevations after the caps were constructed for any of these wells (Figs. 14 through 16). Well 347 , the shallowest of the under-cap wells shows a somewhat larger fluctuation $(>3 \mathrm{~m})$ and occasional artesian conditions prior to completion of cap construction (Fig. 17). Note that this period corresponds to the high-water-level period during cap construction that was evident in the wells outside the capped areas. Although the water level appears to fluctuate less $(\sim 1 \mathrm{~m})$ after cap construction (Fig. 17), this may be an artifact of the reduced frequency of monitoring. The lower water-level elevations subsequent to cap construction are consistent with early 1988 elevations included in Miller et al. (1989), and they probably do not represent an effect of the cap.

\subsection{WELLS INSIDE SWSA 6 BURIAL TRENCHES}

Water levels in intratrench wells (Table A.2, Appendix A) have been monitored for almost 2 years. Results are presented graphically and discussed in the following subsections.

\subsubsection{Cap Area 1 (0.28 ha)}

Only two wells (T82 and T444) within cap area 1 (Fig. 18) contained measurable water during the monitoring period. Contamination of the water-level tape caused us to cease measuring water levels in cap area 1 after the first year.

Well T82 was sampled in April 1989 (Sect. 5). The drawdown associated with this sampling is reflected in the hydrograph (Fig. 19). The water level began to return toward its previous level after the sampling (Fig. 19); however, monitoring was terminated too early to determine whether the water level returned to its precap elevation. The apparent lack of water-level response to rainfall events suggests that trench 444 contains a more-or-less permanent pool of standing water-at least in the vicinity of the well.

Water levels in well T44 showed no significant change after cap construction (Fig. 20).

\subsubsection{Cap Area 2 (0.79 ha)}

In the early 1980s, an L-shaped French drain was installed along the northern and eastern sides of the 49-Trench Area in SWSA 6 (Fig. 1). This drain was effective in reducing water levels in 49-Trench-Area trenches within its zone of effect (Davis et al. 1985) and may have had some effect on the trenches that are now covered by cap area 2 .

Wells T69, T363, and T397 were the only wells in cap area 2 (Fig. 21) that were not dry throughout the monitoring period. All three wells show evidence that cap construction reduced water levels to below the elevation of the trench bottom (Figs. 22 through 24) although occasional rain events still result in water within trench 363 (Fig. 23).

Topographically, cap 2 straddles a small ridge with suriace drainages to the east and west (Fig. 1). One interpretation of the data from the trench wells is that the area covered by cap 2 is a recharge area and is not significantly affected by shallow storm flow from other areas. It is also possible that the French drain and cap act together to lower the shallow aquifer and shutoff storm flow-at least to the trenches (e.g., T69 and T397) within its zone of effect. 


\subsubsection{Cap Area $5(0.38$ ha)}

All of the intratrench wells in cap area 5 (Fig. 25) had measurable water levels during at least some part of the FY 1990 monitoring period. With the exception of well T110, the hydrographs show no significant effect of the cap on either the fluctuation ranges or the elevations of water levels in these trenches (Figs. 26 through 33). Trench 110 appears to have dried out following construction of the cap (Fig. 30).

During the precap period, all trenches were subject to shallow storm flow infiltration from precipitation that falls on or upslope of the cap area. Topographically higher trenches may act to reduce the amount of upslope storm flow that reaches lower trenches. After installation of the cap, the only storm flow that can reach any covered trenches is that originating upgradient of the cap. In cap area 5, it appears that trench 92 may act to reduce upslope storm flow sufficiently to dry out trench 110. However, this effect is not seen on other trenches because sufficient storm flow reaches them from upgridient sources not affected by trench 110 . If trench 92 acts to intercept upslope flow, this phenomenon may also account for trench 92 's apparently greater response to individual precipitation events (Figs. 27 and 28) compared with other trenches both before and after cap construction.

\subsubsection{Cap Area $6(0.61$ ha)}

Four wells (T101, T315, T329, and T395) in cap area 6 (Fig. 34) had measurable water levels during FY 1990. Except for well T329, the water levels were generally at or below the bottom of the trenches except in response to significant precipitation events (Figs. 35 through 38). Well 329 generally had water above the bottom of the trench and responded only slowly to precipitation events (Fig. 37). None of the wells shows a discernible difference in water-level pattern after construction of the cap.

\subsubsection{Cap Area 8 (0.93 ha)}

Nine wells (T44, T60, T63, T180, T225, T237, T352, T367, and T453) in cap area 8 (Fig. 39) had measurable water levels prior to construction of the cap. With the exception of trenches 44 and 63 , the construction of caps 7 and 8 appears to have completely dried out the trenches (Figs. 40-48). The combination of caps 7 and 8 probably covers the recharge area for the cap 8 trenches and eliminates shallow storm flow from this area.

The two trenches that have not completely dried out illustrate an important point. Peak monthly elevations of White Oak Lake (D. M. Borders, ORNL, personal communication, to T. L. Ashwood, ORNL, November 1990) have been plotted on the hydrographs for the cap area 8 wells. Those trenches where the trench bottom is above the highest lake level remain dry throughout the post-cap period. Water levels in trenches 44 and 63 , however, fluctuate directly with the lake level (Figs. 40 and 42). Although other trenches are closer to White Oak Lake than trenches 44 and 63 (Fig. 39), the closer trenches are also shallower (Table A.2, Appendix A), and their bottoms are higher than the lake level.

\section{PENETRATION RESISTANCE OF SWSA 6 BURIAL}

\section{TRENCHES}

As part of the technique for installing monitoring and sampling wells for the capped areas, information on the geotechnical stability of the burial trenches and their contents was obtained. A hole was prepared for insertion of well screen and casing by driving a pointed drill rod with a 
140-lb safety hammer using a cathead hoist for lifting to a standard height of $30 \mathrm{in}$. The technique for driving the pointed drill rod is identical to the standard method for obtaining penetration resistance of soil with a split-spoon sampler (ASTM 1984). Details of operating procedures for this technique, as applied to ORNL SWSA 6 burial trenches, are given in Appendix B. By recording the number of blows of the hammer required to drive each foot of penetration, a measure of the penetration resistance (blows/ft) was obtained. Such penetration resistance measurements have been extensively used in other areas of SWSA 6 for evalu'ting effects of dynamic compaction and in situ grouting on burial trench stability (Spalding et al. 1989).

After withdrawing the drill rod from a burial trench test point, well screen and casing was inserted per the procedures outlined in Appendix B. Schedule-80, polyvinylchloride (PVC), flushjoint, threaded well screen and solid casing (Timco Manufacturing Co., Inc.) was used for construction of the capped area burial trench monitoring wells. Each well consisted of a 5-ft section of slotted $(0.01$ in.) screen fitted with a solid PVC well point. This standard screened section was coupled to sufficient solid casing, in 2- or 5-ft lengths, which extended to above the ground surface. Routinely, 1.5-in. (38-mm) inside diameter casing was used. Use of this diameter casing resulted in a snug fit in the residual test hole such that gentle tapping with the safety hammer was required to achieve insertion. Although the goal was to achieve a fully penetrating well to the bottom of each burial trench, many trenches required more than one penetration test site to attain this depth. Hard objects were regularly encountered during penetrations, which resulted in drill rod refusal. Refusal was defined as less than $1 \mathrm{ft}$ of penetration following 100 blows. The blow counts for refusal (i.e., the bottom fraction of a foot) were not included in the tabulated results or calculations.

Between October 10 and 20,1988, a total of 64 penetration tests were completed in 47 burial trenches in areas that were subsequently capped. A threaded joint in each well casing was placed at or near the ground surface to facilitate placing the surface cover over each capped area. Aboveground extensions of each casing were then reattached following cap placement and cutting of an opening. Sealed boots were later fabricated for all well-casing penetrations so that monitoring and sampling could be carried out after cap installation. However, several wells were lost during construction or damaged beyond repair and, therefore, abandoned.

Penetration tests were also conducted at an additional 25 locations in 9 burial trenches in the group of biological waste disposal trenches west of the tumulus pad. These penetration tests and resulting well locations are in chronically inundated burial trenches that are being used for demonstrations of dynamic compaction, in situ grouting, and trench leachate collection technologies. They have been included with the above penetration tests with capped area trenches because of their similar behavior and because they have provided much information on trench leachate chemical quality that is similar to the trench monitoring wells in capped areas. Most of these penetration tests and well installations were completed in February 1990, although three installations were completed on August 8, 1989. To provide a population of control sites for comparing burial trench penetration tests with stability of the host soil formation, a group of 26 penetration tests, completed in February 1990, have been included (Appendix B). These siies were in the soil formation surrounding trenches 13,279, and 288 in the biological trench area west of the tumulus and trenches 151 and 170 in the 19-trench northeast section, the Test Area for Remedial Actions (TARA). These undisturbed soil formation test results have not been reported previously but are quite similar to those in the undisturbed soil formation in other locations at TARA (Spalding et al. 1989).

The average penetration resistance of test sites in burial trenches, compared with those in the undisturbed soil formation, is depicted in Fig. 49. After the first $2 \mathrm{ft}$ of penetration, where the soil material in the trench covers behaved similar to that in the undisturbed formation, a dramatic decrease in the stability of the burial trenches can be seen. This instability of burial trenches is the primary cause of trench surface subsidence at SWSA 6 and poses a significant compromise to the 
integrity of any cover that might be placed over a group of burial trenches. Although instability had been demonstrated for a group of 19 trenches at the TARA site in SWSA 6 previously (Spalding et al. 1989), such instability has now been clearly established in all burial trenches in SWSA 6. The apparent rise in penetration resistance of builal trenches below $9 \mathrm{ft}$ probably resulted from penetration into the soil formation below many trenches; many of the trenches in wetter areas of SWSA 6 (i.e., caps 5, 6, 8 and the area of biological trenches west of the tumulus) were constructed shallower than the nominal $15 \mathrm{ft}$ of trenches on higher ground. However, because there was no method to verify or determine trench depths, results caused by penetration into the soil formation below the trench floor could not be excluded from the observations. Nonetheless, results still show significantly decreased average penetration resistance below $9 \mathrm{ft}$ regardless of any such effects. Results from the dynamic compaction demonstration at TARA indicate that such differences in penetration resistance would correlate with $\sim 1.3 \mathrm{ft}$ of average surface subsidence over burial trenches compared with that of the surrounding soil formation (Spalding et al. 1989).

To quantify the difference in stability between burial trenches and their surrounding soil formation, cumulative penetration resistance vs depth depicts the difference more clearly (Fig. 50). Over a total depth of $15 \mathrm{ft}$, these burial trenches exhibited about $35 \%$ of the stability of the soil formation. By plotting the ratio of cumulative penetration resistance of the soil formation to that of burial trenches (Fig. 51), it can be seen that stability reaches a minimum about 8 to $9 \mathrm{ft}$ beneath the ground surface, where the ratio is about 0.25 . Not surprisingly, the stability ratio in the top $2 \mathrm{ft}$ of the trench cover is relatively constant. The rising ratio below about $9 \mathrm{ft}$ probably results from an increasing number of tests penetrating into soil below the bottom of shallower trenches. Thus, it is clear that burial trenches in all areas of SWSA 6 are inherently unstable. Stabilization of these trenches prior to capping for infiltration protection will be required to prevent cap compromise.

\section{SAMPLES FROM MONITORING WELLS IN SWSA 6}

\section{BURIAL TRENCHES}

The primary objective of well installation in burial trenches in capped areas was to monitor the response of both seasonally and chronically inundated trenches to the infiltration protection provided by the interim caps. To assess the potential chemical exposures of personnel performing the water-level monitoring in these areas and to supplement the existing information on the quality of leachate in SWSA 6 burial trenches (Solomon et al. 1988), sampling and analyses of trench leachates were initiated. Notably, the entire site characterization of SWSA 6 has avoided actual trench leachate sampling because of concerns over potential hazards to field workers (BNI 1990). However, the value of such leachate chemical quality information in defining source terms and locations for environmental releases is demonstrated by the frequent reference to the previous work of Solomon et al. (1988) in the site characterization report (BNI 1990). In addition to the sampling of wells with standing water in the capped areas, sampling of wells at TARA in burial trenches with transient perched water has been carried out intermittently since 1987. Sampling has also been carried out in nine chronically inundated trenches in the area west of the tumulus in 1989 and 1990. All of the leachate quality data have been assembled here to provide a unified source of this information as a supplement to the site characterization effort.

Sampling of wells in burial trenches in capped areas (trenches 44, 57, 60, 63, 69, 82, 85, 92, $101,105,110,112,180,225,237,318,329,363,367,395,414$, and 453) was carried out between April 5 and 10,1989 after completion of the interim caps. Samples were withdrawn from the well with teflon tubing using a vacuum to pull the sample into a precleaned 1-gal glass jug.

Less than $1 \mathrm{gal}$ of sample was obtained from several wells; as a result not all analyses could be completed on all samples. The vacuum was supplied by an electric vacuum pump fitted with a trap between the sample bottle and the pump. No bailing of standing water in the well casings was 
attempted prior to sampling because of the facile hydraulic connection between standing water and wells in burial trenches (Spalding et al. 1989). Samples were stored at $<4^{\circ} \mathrm{C}$ prior to filtration and submittal for analyses to ORNL Analytical Chemistry Services. Approximately $400 \mathrm{~mL}$ of each sample was transferred to a precleaned polypropylene bottle for analyses of routine gross chemical parameters - $\mathrm{pH}$, electrical conductivity, hardness, alkalinity, dissolved solids, total solids, and gross alpha and beta activities (APHA 1989). After prefiltration through glass wool to remove the frequently occurring suspended solids, the bulk sample was filtered in several batches through $0.22-\mu \mathrm{m}$ Millipore filters. A 1-L subsample of the filtered leachate was placed in a precleaned polypropylene bottle and adjusted to $\mathrm{pH}<2$ with Ultrex nitric acid; this aliquot was submitted for analysis of inorganic elements by inductively coupled plasma (ICP) emission spectroscopy (EPA Method 200.7), and supplemental atomic adsorption spectroscopy was used for analysis of $\mathrm{Hg}, \mathrm{K}$, and Na (EPA Methods SW846-7479M, SW846-7610, and SW846-7770, respectively). A second 1-L aliquot, also stabilized with Ultrex nitric acid, was submitted for radiochemical analyses including gross alpha and beta, tritium, ${ }^{14} \mathrm{C},{ }^{90} \mathrm{Sr}$, and gamma spectroscopy for quantitation of ${ }^{137} \mathrm{Cs}$ and ${ }^{60} \mathrm{Co}$ (EPA Methods 900.0,906.0, 905.0, and 901.1, respectively). A 1-L sample of unfiltered leachate was submitted for analyses of volatile and semivolatile urganic contaminants (EPA Methods 8240 and 8250 , respectively). A $100-\mathrm{mL}$ aliquot of filtered but unpreserved leachate was analyzed for inorganic anions (chloride, fluoride, bromide, phosphate, sulfate, and nitrate) via ion chromatography (EPA Method 300.0), and for total organic carbon (TOC) (EPA Methou SW846-9060). A portion of the unfiltered leachate was analyzed for acrylamide (EPA Method 8015).

Sampling of TARA transient burial trench leachates was achieved by a similar technique but employed a hand vacuum pump to collect sample into eithe: a 250 - or $125-\mathrm{mL}$ precleaned glass vial. Sampling was carried out at various times between 1987 and 1989 (Appendix D). Only one sample from trench 5 on February 26, 1988, exhibited a large gross alpha activity and, therefore, was submitted for alpha spectroscopy to identify its radionuclide composition. Sampling of burial trenches in the area west of the tumulus (trenches 11, 13, 16, 252, 275, 279, 284, 285, and 288) was carried out in 1989 and 1990 using a similar sampling technique.

Results of all the chemical analyses are tabulated in Appendix D, and a summary is presented in Table 1. The summary results have been grouped into five categories: organic analyses, ICP elements, inorganic anions, radionuclides, and gross chemical characteristics. Within each category, species have been ranked by the highest maximum value observed. The frequency of positive findings for a particular species is also listed based on the number of positive findings divided by the number of analyses attempted.

Among the organics, the positively identified compounds were qualitatively the same as those previously identified in trench leachates (Solomon et al. 1988) and in groundwater (BNI 1990). However, the concentrations of ethylbenzene, toluene, and xylene are several orders of magnitude greater than those previously reported. The maximum concentration of ethylbenzene, which was observed in the current population of samples, was greater than its reported water solubility at room temperature (Table 2). The maximal concentrations of xylene and toluene were also within an order of magnitude of their reported water solubilities. All other identified organic compounds were found at concentrations far below their water solubilities. Thus, it appears that concentrations of ethylbenzene, xylene, and toluene may be maintained by the presence of excess solvent in several burial trenches in SWSA 6, although no two-phase samples were observed in the current study. The presence of such aromatic compounds as xylene, toluene, and ethylbenzene (as well as benzene, naphthalene, and microbial breakdown intermediates such as phenol, 4-methylphenol, 2,4- dimethylphenol, and benzoic acid) probably originates from solvents in liquid scintillation cocktail mixtures, which were disposed of in SWSA 6 along with the radioactive solid waste. Similar compounds are also present in petroleum fuels; however, the absence of significant amounts of aliphatic compounds would make a fuel-derived origin unlikely. TOC in the trench 
leachates was also quite elevated over what would be expected for groundwater in SWSA 6. In general, the sum of identified organic species does not account for a substantial fraction of the TOC, except in the high-ethylbenzene, high-toluene, and high-xylene samples. Much of this TOC is probably derived from humified organic matter from the decomposition of the paper and wood products included in the SWSA 6 solid waste.

The presence of acrylamide was also found in 4 of 22 samples examined. It should be pointed out that none of these samples was taken from areas near burial trenches receiving polyacrylamide grout that were used as demonstrations of in situ grouting technology (Spalding et al. 1989). The possibility of any acrylamide contamination resulting from these demonstrations is remote because the 22 samples of leachate were taken from trenches in capped areas prior to the initiation of in situ grouting with polyacrylamide in August 1989. Thus, either the direct injection gas chromatographic method of analysis (EPA Method 8015) produces false positives, or acrylamide is actually present in several trenches in SWSA 6. The latter possibility is plausible because of the potential disposal of polyacrylamide gels, used for electrophoresis anaiyses in biochemical research, or incidental amounts of chemicals (acrylamide) used in their preparation. At the time of these analyses, the more sensitive and less ambiguous method for acrylamide (EPA Method 8032) was not available.

Whereas, the previous sampling of SWSA 6 leachates produced 16 samples from 8 burial trenches, the present group of 42 samples from 26 burial trenches provides a large enough population to allow use of frequency of occurrence of particular compounds to assess the source term of SWSA 6 iontamination. Compounds that occur in greater than $70 \%$ of the samples can be regarded as frequently occurring (Table 1). Organic compounds, including toluene, xylene, acetone, and methylene chloride fall in this frequently occurring category and, thus, merit special attention for potential groundwater remediation or burial trench leachate collection in SWSA 6.

Among the inorganic elements, low concentrations of heavy metals, particularly mercury, were frequently found. Although one anomalously high concentration of phosphorus and one of potassium were encountered, the bulk chemical properties of the trench leachates were generally similar to groundwater in SWSA 6. The average chemical composition of burial trench leachate was similar to that expected for groundwater in contact with limestone (i.e., a calcium and magnesium bicarbonate solution). Occasionally, dissolved iron and manganese were significant contributors to total dissolved cations, presumably resulting from reducing conditions in several of the inundated trenches. Interestingly, lead was always below its detection limit (30 ppb) in all samples analyzed even though lead is generally regarded as a major component of the hazardous substances that have been disposed of in SWSA 6. The solubility of lead, even under the reducing conditions evident in several trenches, must be extremely low.

The radioactivity in burial trench leachates demonstrates the most unique characteristics of SWSA 6 leachates. Tritium and gross beta activity were almost ubiquitously distributed in SWSA 6 burial trench leachates. Occasionally significant gross alpha activity was also observed. In one trench, this gross alpha activity was quite high, $4500 \mathrm{~Bq} / \mathrm{L}$, mostly contributed by $233 \mathrm{U}$ (Table 1).

Among the gross chemical properties, no significant perturbations in $\mathrm{pH}$, electrical conductivity, dissolved solids, hardness, or alkalinity were observed. Thus, significant quantities of watersoluble inorganic chemicals (e.g., acids, bases, and salts) are not present in SWSA 6 burial trench leachates. Suspended solids varied significantly, but because of the presence of loose soil backfill in the trenches, this was not surprising. However, the presence of significant amounts of suspended solids must be recognized as a problem for any leachate collection and treatment technique planned for SWSA 6 closure. 


\begin{tabular}{|c|c|c|c|c|c|c|c|}
\hline Compound & Units & $\begin{array}{l}\text { Number of } \\
\text { positive } \\
\text { findings }\end{array}$ & $\begin{array}{c}\text { Number } \\
\text { of } \\
\text { samples }\end{array}$ & $\begin{array}{l}\text { Average } \\
\text { value }\end{array}$ & 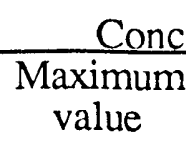 & $\begin{array}{l}\text { Minimution } \\
\text { value }\end{array}$ & $\begin{array}{l}\text { Frequency } \\
\text { of positives }\end{array}$ \\
\hline Ethylbenzene & $\mathrm{ppb}$ & 15 & $\overline{421}$ & $13,559.7$ & 170,000 & 41 & 0.36 \\
\hline Toluene & $\mathrm{ppb}$ & 35 & 421 & $10,881.5$ & 150,000 & 2 & 0.83 \\
\hline Xylene (Total) & ppb & 32 & 421 & 10.071 .5 & 77,000 & 1 & 0.76 \\
\hline Acetone & $\mathrm{ppb}$ & 37 & 42 & $3,750.2$ & 50,000 & 1 & 0.88 \\
\hline Naphthalene & $\mathrm{ppb}$ & 20 & 33 & $1,719.9$ & 8,800 & 18 & 0.61 \\
\hline 4-Methylphenol & $\mathrm{ppb}$ & 14 & 33 & $1,173.6$ & 4,600 & 16 & 0.42 \\
\hline Acrylamide & $\mathrm{ppb}$ & 4 & 22 & 1,745 & 3,800 & 90 & 0.18 \\
\hline Methylene chloride & $\mathrm{ppb}$ & 30 & 42 & 320.8 & 2,900 & 3 & 0.71 \\
\hline Benzoic acid & $\mathrm{ppb}$ & 10 & 33 & 460.1 & 2,400 & 4 & 0.3 \\
\hline Phenol & $\mathrm{ppb}$ & 11 & 33 & 323.5 & 1,800 & 2 & 0.33 \\
\hline Carbon disulfide & $\mathrm{ppb}$ & 3 & 42 & 297.1 & 890 & 0.6 & 0.07 \\
\hline Chloroform & $\mathrm{ppb}$ & 11 & 42 & 58.9 & 560 & 0.6 & 0.26 \\
\hline 2-Methylnaphthalene & $\mathrm{ppb}$ & 2 & 33 & 2.22 & 420 & 24 & 0.06 \\
\hline Nitrobenzene & $\mathrm{ppb}$ & 1 & 33 & 410 & 410 & 410 & 0.03 \\
\hline 2,4-Dimethylphenol & $\mathrm{ppb}$ & 8 & 33 & 136.8 & 380 & 17 & 0.24 \\
\hline Benzene & $\mathrm{ppb}$ & 12 & 42 & 38 & 240 & 0.6 & 0.29 \\
\hline 2-Methylphenol & ppb & 7 & 33 & 55.7 & 200 & 15 & 0.21 \\
\hline 2-Butanone & $\mathrm{ppb}$ & 5 & 42 & 30.5 & 95 & 0.5 & 0.12 \\
\hline Trichloroethene & $\mathrm{ppb}$ & 5 & 42 & 13 & 36 & 4 & 0.12 \\
\hline Styrene & $\mathrm{ppb}$ & 1 & 42 & 23 & 23 & 23 & 0.02 \\
\hline Diethylphthalate & $\mathrm{ppb}$ & 1 & 33 & 12 & 12 & 12 & 0.03 \\
\hline Di-n-butylphthalate & ppb & 5 & 33 & 2.4 & 5 & 1 & 0.15 \\
\hline 4-Methyl-2-pentanone & $\mathrm{ppb}$ & 1 & 42 & 3 & 3 & 3 & 0.02 \\
\hline 1,2-Dichloroethane & $\mathrm{ppb}$ & 1 & 42 & 3 & 3 & 3 & 0.02 \\
\hline Hexachlorobutadiene & $\mathrm{ppb}$ & 0 & 33 & $<10$ & & & 0 \\
\hline 2,4,5-Trichlorophenol & $\mathrm{ppb}$ & 0 & 33 & $<50$ & & & 0 \\
\hline 2-Chloronaphthalene & $\mathrm{ppb}$ & 0 & 33 & $<10$ & & & 0 \\
\hline trans-1,3-Dichloropropene & $\mathrm{ppb}$ & 0 & 42 & $<5$ & & & 0 \\
\hline 2-Nitroaniline & $\mathrm{ppb}$ & 0 & 33 & $<50$ & & & 0 \\
\hline Tetrachloroethene & $\mathrm{ppb}$ & 0 & 42 & $<5$ & & & 0 \\
\hline Dimethylphthalate & $\mathrm{ppb}$ & 0 & 33 & $<10$ & & & 0 \\
\hline 1,1,1-Trichloroethane & $\mathrm{ppb}$ & 0 & 42 & $<5$ & & & 0 \\
\hline Acenaphthylene & $\mathrm{ppb}$ & 0 & 33 & $<10$ & & & 0 \\
\hline $1,1,2$-Trichloroethane & $\mathrm{ppb}$ & 0 & 42 & $<5$ & & & 0 \\
\hline 2,6-Dinitrotoluene & $\mathrm{ppb}$ & 0 & 33 & $<10$ & & & 0 \\
\hline cis-1,3-Dichloropropene & $\mathrm{ppb}$ & 0 & 42 & $<5$ & & & 0 \\
\hline 3-Nitroaniline & $\mathrm{ppb}$ & 0 & 33 & $<50$ & & & 0 \\
\hline bis(2-Chloroethyl)ether & $\mathrm{ppb}$ & 0 & 33 & $<10$ & & & 0 \\
\hline Acenaphthene & $\mathrm{ppb}$ & 0 & 33 & $<10$ & & & 0 \\
\hline 1,3-Dichlorobenzene & $\mathrm{ppb}$ & 0 & 33 & $<10$ & & & 0 \\
\hline 2,4-Dinitrophenol & $\mathrm{ppb}$ & 0 & 33 & $<50$ & & & 0 \\
\hline Benzyl alcohol & $\mathrm{ppb}$ & 0 & 33 & $<10$ & & & 0 \\
\hline 4-Nitrophenol & $\mathrm{ppb}$ & 0 & 33 & $<50$ & & & 0 \\
\hline 1,2-Dichloropropane & $\mathrm{ppb}$ & 0 & 42 & $<5$ & & & 0 \\
\hline Dibenzofuran & $\mathrm{ppb}$ & 0 & 33 & $<10$ & & & 0 \\
\hline 1,1-Dichloroethane & $\mathrm{ppb}$ & 0 & 42 & $<5$ & & & 0 \\
\hline
\end{tabular}


Table 1 (continued) Summary of chemical analyses of SWSA 6 burial trench leachate samples

\begin{tabular}{|c|c|c|c|c|c|c|}
\hline Compound & Units & $\begin{array}{l}\text { Number of } \\
\text { positive } \\
\text { findings }\end{array}$ & $\begin{array}{l}\text { Number } \\
\text { of } \\
\text { samples }\end{array}$ & $\begin{array}{l}\text { A.verage } \\
\text { value }\end{array}$ & $\begin{array}{c}\text { Concentration } \\
\text { Maximum Minimum } \\
\text { value value }\end{array}$ & $\begin{array}{l}\text { Frequency } \\
\text { of positives }\end{array}$ \\
\hline 2,4-Dinitrotoluene & $\mathrm{ppb}$ & 0 & 33 & $<10$ & & 0 \\
\hline Hexachloroethane & $\mathrm{ppb}$ & 0 & 33 & $<10$ & & 0 \\
\hline Bromodichloromethane & $\mathrm{ppb}$ & 0 & 42 & $<5$ & & 0 \\
\hline Isophorone & $\mathrm{ppb}$ & 0 & 33 & $<10$ & & 0 \\
\hline 4-Chlorophenyl-phenylether & rppb & 0 & 33 & $<10$ & & 0 \\
\hline Chloromethane & $\mathrm{ppb}$ & 0 & 42 & $<10$ & & 0 \\
\hline Fluorene & ppb & 0 & 33 & $<10$ & & 0 \\
\hline bis(2-chloroethoxy)methane & ppb & 0 & 33 & $<10$ & & 0 \\
\hline 4-Nitroaniline & $\mathrm{ppb}$ & 0 & 33 & $<50$ & & 0 \\
\hline 1,2,4-Trichlorobenzene & $\mathrm{ppb}$ & 0 & 33 & $<10$ & & 0 \\
\hline 4,6-Dinitro-2-methylphenol & $\mathrm{ppb}$ & 0 & 33 & $<50$ & & 0 \\
\hline 4-chloroaniline & $\mathrm{ppb}$ & $\hat{0}$ & 33 & $<10$ & & 0 \\
\hline $\mathrm{N}$-nitrosodiphenylamine & $\mathrm{ppb}$ & 0 & 33 & $<10$ & & 0 \\
\hline 4-Chloro-3-methylphenol & $\mathrm{ppb}$ & 0 & 33 & $<10$ & & 0 \\
\hline 4-bromophenyl-phenylether & $\mathrm{ppb}$ & 0 & 33 & $<10$ & & 0 \\
\hline Hexachlorocyclopentadiene & $\mathrm{ppb}$ & 0 & 33 & $<10$ & & 0 \\
\hline Hexachlorobenzene & $\mathrm{ppb}$ & 0 & 33 & $<10$ & & 0 \\
\hline Bromoform & $\mathrm{ppb}$ & 0 & 42 & $<5$ & & 0 \\
\hline Pentachlorophenol & $\mathrm{ppb}$ & 0 & 33 & $<50$ & & 0 \\
\hline 1,1,2,2-tetrachloroethane & $\mathrm{ppb}$ & 0 & 42 & $<5$ & & 0 \\
\hline Phenanthrene & $\mathrm{ppb}$ & 0 & 33 & $<10$ & & 0 \\
\hline Dibromochloromethane & $\mathrm{ppb}$ & 0 & 42 & $<5$ & & 0 \\
\hline Anthracene & $\mathrm{ppb}$ & 0 & 33 & $<10$ & & 0 \\
\hline 2-Chlorophenol & ppb & 0 & 33 & $<10$ & & 0 \\
\hline Vinyl acetate & $\mathrm{ppb}$ & 0 & 42 & $<10$ & & 0 \\
\hline 1,2-Dichlorobenzene & $\mathrm{ppb}$ & 0 & 33 & $<10$ & & 0 \\
\hline Fluoranthene & $\mathrm{ppb}$ & 0 & 33 & $<10$ & & 0 \\
\hline $\mathrm{N}$-nitroso-di-n-propylamine & $\mathrm{ppb}$ & 0 & 33 & $<10$ & & 0 \\
\hline Pyrene & $\mathrm{ppb}$ & 0 & 33 & $<10$ & & 0 \\
\hline 2-Nitrophenol & ppb & 0 & 33 & $<10$ & & 0 \\
\hline Butylbenzylphthalate & $\mathrm{ppb}$ & 0 & 33 & $<10$ & & 0 \\
\hline 2,4-Dichlorophenol & $\mathrm{ppb}$ & 0 & 33 & $<10$ & & 0 \\
\hline 3,3'-Dichlorobenzidene & ppb & 0 & 33 & $<20$ & & 0 \\
\hline Carbon tetrachloride & $\mathrm{ppb}$ & 0 & 42 & $<5$ & & 0 \\
\hline Benzo(a)anthracene & ppb & 0 & 33 & $<10$ & & 0 \\
\hline 2,4,6-Trichlorophenol & $\mathrm{ppb}$ & 0 & 33 & $<10$ & & 0 \\
\hline Chrysene & ppb & 0 & 33 & $<10$ & & 0 \\
\hline Chlorobenzene & $\mathrm{ppb}$ & 0 & 42 & $<5$ & & 0 \\
\hline bis(2-Ethylhexyl)phthalate & $\mathrm{ppb}$ & 0 & 33 & $<10$ & & 0 \\
\hline 1,4-Dichlorobenzene & ppb & 0 & 33 & $<10$ & & 0 \\
\hline Di-n-Octylphthalate & $\mathrm{ppb}$ & 0 & 33 & $<10$ & & 0 \\
\hline 1,1-Dichloroethene & $\mathrm{ppb}$ & 0 & 42 & $<5$ & & 0 \\
\hline Benzo(b)fluoranthene & $\mathrm{ppb}$ & 0 & 33 & $<10$ & & 0 \\
\hline Vinyl Chloride & $\mathrm{ppb}$ & 0 & 42 & $<10$ & & 0 \\
\hline Benzo(k)fluoranthene & $\mathrm{ppb}$ & 0 & 33 & $<10$ & & 0 \\
\hline 2-Hexanone & ppb & 0 & 42 & $<10$ & & 0 \\
\hline
\end{tabular}


Table 1 (continued) Summary of chemical analyses of SWSA 6 burial trench leachate samples

\begin{tabular}{|c|c|c|c|c|c|c|c|}
\hline Compound & Units & $\begin{array}{l}\text { Number of } \\
\text { positive } \\
\text { findings }\end{array}$ & $\begin{array}{l}\text { Numbe } \\
\text { of } \\
\text { samples }\end{array}$ & $\begin{array}{l}\text { Average } \\
\text { es value }\end{array}$ & $\begin{array}{c}\text { Conc } \\
\text { Maximum } \\
\text { value }\end{array}$ & 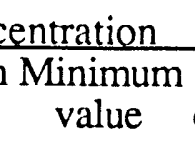 & $\begin{array}{l}\text { Frequency } \\
\text { of positives }\end{array}$ \\
\hline Benzo(a)pyrene & $\mathrm{ppb}$ & 0 & 33 & $<10$ & & & 0 \\
\hline bis(2-Chloroisopropyl)ether & $\mathrm{rppb}$ & 0 & 33 & $<10$ & & & 0 \\
\hline Indeno(1,2,3-cd)pyrene & $\mathrm{ppb}$ & 0 & 33 & $<10$ & & & 0 \\
\hline Bromomethane & $\mathrm{ppb}$ & 0 & 42 & $<10$ & & & 0 \\
\hline Chloroethane & $\mathrm{ppb}$ & 0 & 42 & $<10$ & & & 0 \\
\hline 1,2-Dichloroethene (total) & $\mathrm{ppb}$ & 0 & 42 & $<5$ & & & 0 \\
\hline Dibenz $(a, h)$ anthracene & $\mathrm{ppb}$ & 0 & 33 & $<10$ & & & 0 \\
\hline Benzo(g,h,i)perylene & $\mathrm{ppb}$ & 0 & 33 & $<10$ & & & 0 \\
\hline Phosphorus & $\mathrm{ppb}$ & 2 & 30 & 93,500 & 140,000 & 47,000 & 0.07 \\
\hline Calcium & ppb & 30 & 30 & $72,066.7$ & 140,000 & 20,000 & 1 \\
\hline Potassium & $\mathrm{ppb}$ & 28 & 30 & $8,504.6$ & 110,000 & 530 & 0.93 \\
\hline Silicon & $\mathrm{ppb}$ & 30 & 30 & $5,629.3$ & 67,000 & 410 & 1 \\
\hline Sodium & $\mathrm{ppb}$ & 26 & 30 & $9,508.8$ & 50,200 & 1,040 & 0.87 \\
\hline Magnesium & $\mathrm{ppb}$ & 30 & 30 & 17,500 & 40,000 & 4,500 & 1 \\
\hline Iron & $\mathrm{ppb}$ & 23 & 30 & $8,929.9$ & 40,000 & 10 & 0.77 \\
\hline Manganese & $\mathrm{ppb}$ & 30 & 30 & $6,741.1$ & 36,000 & 11 & 1 \\
\hline Mercury & $\mathrm{ppb}$ & 19 & 30 & 112.5 & 2,120 & 0.1 & 0.63 \\
\hline Nickel & $\mathrm{ppb}$ & 2 & 30 & 645 & 1,100 & $190^{\circ}$ & 0.07 \\
\hline Zinc & $\mathrm{ppb}$ & 15 & 30 & 148.9 & 560 & 8 & 0.5 \\
\hline Barium & $\mathrm{ppb}$ & 30 & 30 & 228.4 & 550 & 61 & 1 \\
\hline Aluminum & $\mathrm{ppb}$ & 14 & 30 & 292.9 & 510 & 130 & 0.47 \\
\hline Cadmium & $\mathrm{ppb}$ & 2 & 30 & 147.5 & 290 & 5 & 0.07 \\
\hline Strontium & $\mathrm{ppb}$ & 30 & 30 & 126.2 & 210 & 41 & 1 \\
\hline Boron & $\mathrm{ppb}$ & 1 & 30 & 160 & 160 & 160 & 0.03 \\
\hline Antimony & $\mathrm{ppb}$ & 4 & 30 & 70.5 & 100 & 51 & 0.13 \\
\hline Arsenic & $\mathrm{ppb}$ & 8 & 30 & 67.9 & 87 & 54 & 0.27 \\
\hline Selenium & $\mathrm{ppb}$ & 2 & 30 & 70 & 81 & 59 & 0.07 \\
\hline Cobalt & ppb & 23 & 30 & 22.9 & 49 & 4 & 0.77 \\
\hline Chromium & $\mathrm{ppb}$ & 28 & 30 & 11.8 & 43 & 3 & 0.93 \\
\hline Copper & $\mathrm{ppb}$ & 7 & 30 & 22.4 & 29 & 13 & 0.23 \\
\hline Silver & $\mathrm{ppb}$ & 16 & 30 & 9.9 & 15 & 5 & 0.53 \\
\hline Tin & $\mathrm{ppb}$ & 0 & 30 & $<50$ & & & 0 \\
\hline Titanium & $\mathrm{ppb}$ & 0 & 30 & $<20$ & & & 0 \\
\hline Vanadium & $\mathrm{ppb}$ & 0 & 30 & $<4$ & & & 0 \\
\hline Lithium & $\mathrm{ppb}$ & 0 & $30<$ & 15,000 & & & 0 \\
\hline Molybdenum & $\mathrm{ppb}$ & 0 & 30 & $<40$ & & & 0 \\
\hline Lead & $\mathrm{ppb}$ & 0 & 30 & $<30$ & & & 0 \\
\hline Beryllium & $\mathrm{ppb}$ & 0 & 30 & $<0.4$ & & & 0 \\
\hline Zirconium & $\mathrm{ppb}$ & 0 & 30 & $<20$ & & & 0 \\
\hline Total organic carbon & ppb & 30 & $30 \varepsilon$ & 82,150 & 615,000 & 1,400 & 1 \\
\hline Phosphate & $\mathrm{ppb}$ & 6 & 30 & $60,111.7$ & 294,000 & 60 & 0.2 \\
\hline Fluoride & $\mathrm{ppb}$ & 25 & 30 & $6,861.2$ & 63,300 & 140 & 0.83 \\
\hline Sulfate & $\mathrm{ppb}$ & 30 & 301 & $12,447.7$ & 61,900 & 280 & 1 \\
\hline Chloride & $\mathrm{ppb}$ & 30 & 301 & $12,063.3$ & 51,700 & 1,150 & 1 \\
\hline Nitrate & $\mathrm{ppb}$ & 28 & 30 & 902.1 & 6,690 & 60 & 0.93 \\
\hline Bromide & $\mathrm{ppb}$ & 8 & 30 & 96.3 & 170 & 60 & 0.27 \\
\hline
\end{tabular}


Table 1 (continued). Summary of chemical analyses of SWSA 6 burial trench leachate samples

\begin{tabular}{|c|c|c|c|c|c|c|c|}
\hline Compound & $\begin{array}{r}\mathrm{N} \\
\text { Units } \\
\mathrm{f}\end{array}$ & $\begin{array}{l}\text { Number of } \\
\text { positive } \\
\text { findings }\end{array}$ & $\begin{array}{c}\text { Number } \\
\text { of } \\
\text { samples }\end{array}$ & $\begin{array}{l}\text { Average } \\
s \text { value }\end{array}$ & 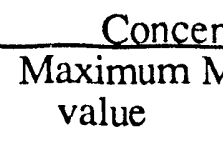 & $\frac{\text { ntration }}{\text { Minimum } \mathrm{F}}$ & $\begin{array}{l}\text { Frequency } \\
\text { of positives }\end{array}$ \\
\hline Tritium & $\mathrm{Bq} / \mathrm{L}$ & 41 & 422 & $22,283.1$ & 850,000 & 32 & 0.98 \\
\hline Gross Beta & $\mathrm{Bq} / \mathrm{L}$ & 44 & 46 & $8,450.7$ & 346,736 & 0.01 & 0.96 \\
\hline Gross Alpha & $\mathrm{Bq} / \mathrm{L}$ & 29 & 46 & 282.5 & 4,500 & 0.029 & 0.63 \\
\hline Uranium-233 & $\mathrm{Bq} / \mathrm{L}$ & 1 & 1 & 3,700 & 3,700 & 3,700 & 1 \\
\hline Strontium-90 & $\mathrm{Bq} / \mathrm{L}$ & 24 & 24 & 30.4 & 660 & 0.01 & 1 \\
\hline Uranium-232 & $\mathrm{Bq} / \mathrm{L}$ & 1 & 1 & 58 & 58 & 58 & 1 \\
\hline Carbon-14 & $\mathrm{Bq} / \mathrm{L}$ & 20 & 24 & 10.5 & 38 & 1 & 0.83 \\
\hline Cesium-137 & $\mathrm{Bq} / \mathrm{L}$ & 14 & 24 & 7.9 & 36 & 0.2 & 0.58 \\
\hline Cobalt -60 & $\mathrm{~Bq} / \mathrm{L}$ & 6 & 24 & 0.8 & 1.6 & 0.1 & 0.25 \\
\hline Americium-241 & $\mathrm{Bq} / \mathrm{L}$ & 1 & 1 & 1.1 & 1.1 & 1.1 & 1 \\
\hline Plutonium-239 & $\mathrm{Bq} / \mathrm{L}$ & 1 & 1 & 0.8 & 0.8 & 0.8 & 1 \\
\hline Curium-244 & $\mathrm{Bq} / \mathrm{L}$ & C & 1 & $<0.1$ & & & 0 \\
\hline $\mathrm{pH}$ & $-\log (\mathrm{H}+)$ & 58 & 58 & 7.3 & 8.55 & 5.5 & 1 \\
\hline Electrical conductivity & $\mathrm{dS} / \mathrm{m}$ & 58 & 58 & 787.7 & 8,580 & 55 & 1 \\
\hline Dissolved solids & $\mathrm{mg} / \mathrm{L}$ & 59 & 59 & 521.9 & 6,360 & 20 & 1 \\
\hline Total solids & $\mathrm{mg} / \mathrm{L}$ & 34 & 34 & $3,702.4$ & 26,020 & 100 & 1 \\
\hline Suspended solids & $\mathrm{mg} / \mathrm{L}$ & 34 & 34 & 3,252 & 25,820 & $<1$ & 1 \\
\hline Hardness & $\mathrm{mg} / \mathrm{L}$ & 58 & 58 & 347 & 5,220 & 16 & 1 \\
\hline Alkalinity & $\mathrm{mg} / \mathrm{L}$ & 58 & 58 & 461.1 & 6,511 & 21 & 1 \\
\hline
\end{tabular}

Table 2. Water solubilities of organic compounds in SWSA 6 burial trench leachate samples ${ }^{a}$

\begin{tabular}{lcr}
\hline \multicolumn{1}{c}{ Compound } & CAS No. & $\begin{array}{c}\text { Water } \\
\text { solubility } \\
\text { (ppb) }\end{array}$ \\
\hline Chloromethane & $74-87-3$ & not found \\
Bromomethane & $74-83-9$ & 900,000 \\
Vinyl Chloride & $75-01-4$ & 1,100 \\
Chloroethane & $75-00-3$ & $5,740,000$ \\
Methylene chloride & $75-09-2$ & $20,000,000$ \\
Acetone & $67-64-1$ & miscible \\
Miscible Carbon disulfide & $75-15-0$ & $2,300,000$ \\
1,1-Dichloroethene & $75-35-4$ & $2,640,000$ \\
1,1-Dichloroethane & $75-34-3$ & $5,500,000$ \\
1,2-Dichloroethene (total) & $540-59-0$ & 800,000 \\
Chloroform & $67-66-3$ & $9,300,000$ \\
1,2-Dichloroethane & $107-06-2$ & $8,690,000$ \\
2-Butanone & $78-93-3$ & $350,000,000$ \\
1,1,1-Trichloroethane & $71-55-6$ & $4,400,000$ \\
Carbon tetrachloride & $56-23-5$ & $1,160,000$
\end{tabular}


Table 2 (continued) Water soiubilities of organic compounds in SWSA 6 burial trench leachate samplesa

\begin{tabular}{|c|c|c|}
\hline Compound & CAS No. & $\begin{array}{l}\text { Water } \\
\text { solubility } \\
\text { (ppb) }\end{array}$ \\
\hline Vinyl acetate & $108-05-4$ & $25,000,000$ \\
\hline Bromodichloromethane & $75-27-4$ & not found \\
\hline 1,2-Dichloropropane & $78-87-5$ & $2,700,000$ \\
\hline cis-1,3-Dichloropropene & $10061-01-5$ & $2,700,000$ \\
\hline Trichloroethene & $79-01-6$ & $1,100,000$ \\
\hline Dibromochloromethane & $124-48-1$ & not found \\
\hline 1,1,2-Trichloroethane & $79-00-5$ & $4,500,000$ \\
\hline Benzene & $71-43-2$ & $1,780,000$ \\
\hline trans-1,3-Dichloropropene & $10061-02-06$ & $2,800,000$ \\
\hline Bromoform & $75-25-2$ & $3,190,000$ \\
\hline 4-Methyl-2-pentanone & $108-10-1$ & $17,000,000$ \\
\hline 2-Hexanone & $591-78-6$ & $35,000,000$ \\
\hline Tetrachloroethene & $127-18-4$ & 150,000 \\
\hline 1,1,2,2-tetrachloroethane & $79-34-5$ & $2,900,000$ \\
\hline Toluene & $108-88-3$ & 515,000 \\
\hline Chlorobenzene & $108-90-7$ & 500,000 \\
\hline Ethylbenzene & $100-41-4$ & 152,000 \\
\hline Styrene & $100-42-5$ & 300,000 \\
\hline Xylene (Total) & $1330-20-7$ & 198,000 \\
\hline Phenol & $108-95-2$ & $82,000,000$ \\
\hline bis(2-Chloroethyl)ether & $111-44-4$ & $10,200,000$ \\
\hline 2-Chlorophenol & $95-57-8$ & $28,500,000$ \\
\hline 1,3-Dichlorobenzene & $541-73-1$ & 123,000 \\
\hline 1,4-Dichlorobenzene & $106-46-7$ & 79,000 \\
\hline Benzyl alcohol & $100-51-6$ & $35,000,000$ \\
\hline 1,2-Dichlorobenzene & $95-50-1$ & 100,000 \\
\hline 2-Methylphenol & $95-48-7$ & $31,000,000$ \\
\hline bis(2-Chloroisopropyl)ether & $108-60-1$ & $1,700,000$ \\
\hline 4-Methylphenol & $106-44-5$ & $24,000,000$ \\
\hline N-nitroso-di-n-propylamine & $621-64-7$ & not found \\
\hline Hexachloroethane & $67-72-1$ & 50,000 \\
\hline Nitrobenzene & $98-95-3$ & $1,900,000$ \\
\hline Isophorone & $78-59-1$ & $12,000,000$ \\
\hline 2-Nitrophenol & $88-75-5$ & $2,100,000$ \\
\hline 2,4-Dimethylphenol & $105-67-9$ & $>500,000$ \\
\hline Benzoic acid & $65-85-0$ & $2,900,000$ \\
\hline bis(2-chloroethoxy)methane & $111-91-1$ & not found \\
\hline 2,4-Dichlorophenol & $120-83-2$ & $4,600,000$ \\
\hline 1,2,4-Trichlorobenzene & $120-82-1$ & 19,000 \\
\hline Naphthalene & $91-20-3$ & 34,000 \\
\hline 4-chloroaniline & $106-47-8$ & 340,000 \\
\hline Hexachlorobutadiene & $87-68-3$ & 2,000 \\
\hline 4-Chloro-3-methylphenol & $59-50-7$ & $3,846,000$ \\
\hline 2-Methylnaphthalene & $91-57-6$ & 12 \\
\hline Hexachlorocyclopentadiene & $77-47-4$ & not found \\
\hline
\end{tabular}


Table 2 (cont.). Water solubilities of organic compounds in SWSA 6 burial trench leachate samplesa

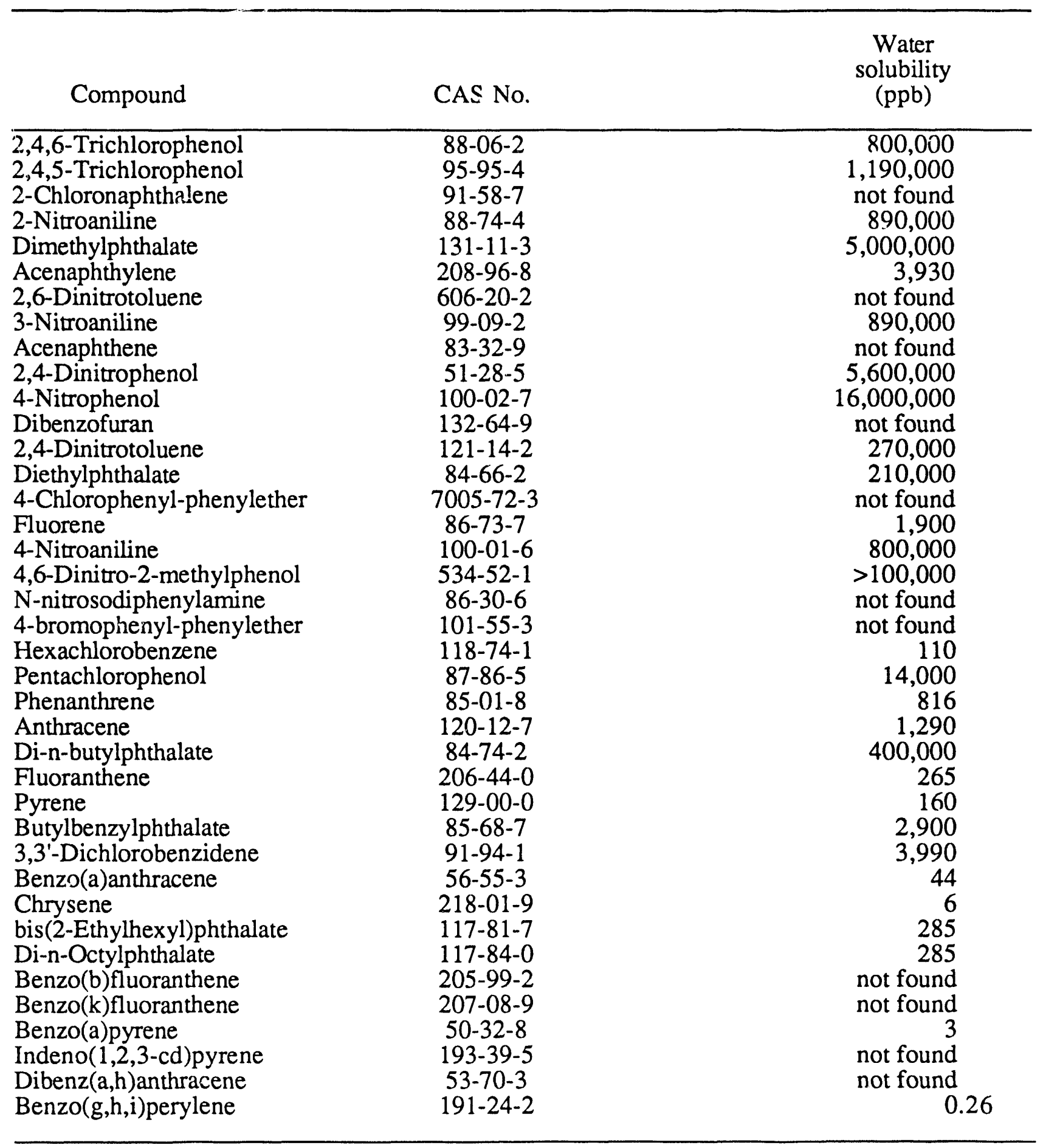

a Data from Verschueren (1983). 


\section{CONCLUSIONS}

The evaluations of water-level changes in this renort have been semiquantitative because of the relatively low frequency of observations and the lack of data to quantitatively evaluate changes in the water budget for SWSA 6 (i.e., no measurement of surface water discharge and no measurement of direct groundwater discharge to White Oak Lake). Nevertheless, within the constraints of a semiquantitative evaluation, it is possible to infer several important conclusions. These conclusions can have a significant impact on the choice of remedial actions and strategies for closure of SWSA 6. In those cases in which readers feel that uncertainties or gaps in our data are too great to provide unequivocal support for the conclusions, they may want to obtain additional data.

The ICM caps have apparently had very little, if any, observable effect on groundwater levels outsicle the capped areas. Only well 318 , which is west and down slope of cap area 2, shows any notice a'sle difference between precap and postcap water-level patterns. It is possible that cap 2 covers a large portion of the recharge zone for shallow storm flow to well 318. Additional review of early water-level data from this well and additional monitoring are needed to clearly ascertain whether it is the cap or another hydrologic process that is causing the change in well 318.

It is not surprising that the caps have had little effect on the wells outside their boundaries. Some of the wells are in locations that would not be expected to be affected by the caps. Those wells within the possible zone of influence of the caps are typically measuring water levels in the aquifer below SWSA 6 . Recharge of this aquifer is apparently not significantly affected by the caps, probaibly because they do not cover a significant portion of the recharge zone.

With the possible exception of well 347 under cap 5, none of the groundwater wells beneath caps showed any discernible impact from cap construction. As with the wells outside the caps, these under-cap wells are primarily measuring fluctuations in the aquifer, which appears to be largely unaffected by the caps.

Another reason that the aquifer below SWSA 6 does not appear to be affected by the caps may be that water accumulating in the trenches as a result of shallow storm flow (i.e., bathtubbing trenches) can slowly percolate out of the trenches and recharge the aquifer. Only cap areas 2 and 8 appear to have reduced this bathtubbing effect significantly. Trenches in other capped areas still provide a path for shallow storm flow to recharge the aquifer. Given the poor chemical quality of trench leachates, this source of recharge is also a source of contamination to the aquifer.

Caps 2 and 8 appear to have been successful in reducing the perched water within the trenches beneath them. In both areas, the caps probably cover a major portion of the recharge zone for shallow storm flow. Caps 1,5 , and 6 have had no observable impact on water levels within their associated trenches. No data are available for trenches under caps 3 and 7.

Two trenches under cap 8 are responding directly to fluctuations in White Oak Lake levels. When the lake elevation is above the bottom of these trenches, water enters the trenches. The 100 -year flood elevation is $-230 \mathrm{~m}$ (754 ft) (BNI 1988). This level is higher than the bottom of most trenches under cap 8 and possibly other areas oi SWSA 6 . The implications of this phenomenon for closure of SWSA 6 are significant because it means that an effective cap alone may not prevent groundwater from interacting with buried wastes in areas below the elevation of White Oak Lake. Some means of isolating these trenches from the lake may be necessary.

The stability of the SWSA 6 burial trenches was found to be only $35 \%$ that of the surrounding soil formation. This conclusion is based on penetration resistance tests performed at 89 locations within 56 SWSA 6 burial trenches. Thus, significant differential land surface subsidence can be 
expected for unstabilized burial trenches in SWSA 6, and their capacity to support infiltration barriers over the long term must be carefully evaluated.

Significant concentrations of ethylbenzene, toluene, and xylene (approaching their water solubilities) were measured in several ieachate samples from SWSA 6 burial trenches. Toluene, xylene, acetone, and methylene chloride were detected in more than $70 \%$ of the 42 trench leachates sampled. Tritium and gross beta activity were observed in more than $90 \%$ of the leachate samples. Many concentrations of contaminants were significantly higher, in some cases by several orders of magnitude, than those reported in previous limited sampling of SWSA 6 leachates. This new data on leachate chemical quality should aid in determining source term characteristics for performance modeling of SWSA 6.

\section{ACKNOWLEDGMENTS}

We would like to thank Gregory Miller and others at Environmental Consulting Engineers, Inc., who initiated a portion of this program and helped in the transition of data collection and reporting responsibilities. David Farmer and Della Marshall measured water levels and collected samples. Mike Morrissey provided rainfall data. Dennis Borders provided data on White Oak Lake elevations. Ed Davis did much of the early field work as well as the surveying and computer mapping of the intratrench wells. Dale Huff and Chet Francis provided valuable comments on a draft of this report.

\section{REFERENCES}

American Public Health Association (APHA). 1989. Standard Methods for the Examination of Water and Wastewater. American Public Health Association, Washington, D. C.

American Society for Testing Materials (ASTM). 1984. Standard Method for Penetration Test and Split-Barrel Sampling of Soils. D 1586-84. Philadelphia, Pa.

Ashwood, T. L., D. S. Wickliff, and C. M. Morrissey. 1990. Active sites environmental monitoring program: program plan. ORNL/M-1197. Oak Ridge National Laboratory, Oak Ridge, Tenn.

Bechtel National, Inc. (BNI). 1988. Closure plan for solid waste storage area 6. ORNL/RAP/Sub-87/99053/9\&V1. Oak Ridge National Laboratory, Oak Ridge, Tenn.

Bechtel National, Inc. (BNI) 1990. ORNL WAG 6 Site Characterization Summary. Volume I. Main Report. ORNL/ER-11/V1\&D1. Oak Ridge National Laboratory, Oak Ridge, Tenn.

Boegly, W. J. 1984. Site characterization data for Solid Waste Storage Area 6. ORNL/TM-9442. Oak Ridge National Laboratory, Oak Ridge, Tenn.

Boegly, W. J., R. B. Dreier, D. D. Huff, A. D. Kelmers, D. C. Kocher, S. Y. Lee, F. R. O'Donnell, F. G. Pin, and E. D. Smith. 1985. Characterization plan for Solid Waste Storage Area 6. ORNL/TM-9877. Oak Ridge National Laboratory, Oak Ridge, Tenn.

Davis, E. C., R. G. Stansfield, L. A. Melroy, and D. D. Huff. 1985. Water diversion at low-level waste disposal sites. J. Environ. Eng. 111(5):714-29.

Hook, L. A., L. ' . Voorhees, M. J. Gentry, M. A. Faulkner, J. A. Shaakir-Ali, K. A. Newman, R. A. McCord, L. F. Goins, and P. T. Owen. 1990. Database management activities for the remedial action program at ORNL: Calendar year 1989. ORNL/ER-16. Oak Ridge National Laboratory, Oak Ridge, Tenn. 
Kimbrough, C. W., L. W. Long, and L. W. McMahon. 1990. Environmental Surveillance Procedures Quality Control Program. ESH/Sub/87-21706/1 Rev. 1. Martin Marietta Energy Systems, Inc., Oak Ridge, Tenn.

Miller, G. P., and P. M. Craig. 1988. Solid Waste Storage Area 6 interim corrective measures environmental monitoring plan. ECE-88-022. Environmental Consulting Engineers, Inc., Knoxville, Tenn.

Miller, G. P., K. C. Black, and P. M. Craig. 1989. SWSA-6 interim corrective measures environmental monitoring summary report. ECE-89-017. Environmental Consulting Engineers, Inc., Knoxville, Tenn.

Moore, G. K. 1988. Concepts of groundwater occurrence and flow near Oak Ridge National Laboratory, Tennessee. ORNL/TM-10969. Oak Ridge National Laboratory, Oak Ridge, Tenn.

Moore, G. K. 1989. Groundwater parameters and flow systems near Oak Ridge National Laboratory. ORNL/TM-11368. Oak Ridge National Laboratory, Oak Ridge, Tenn.

Solomon, D. K., R. C. Haese, T. V. Dinsmore, A. D. Kelmers. 1988. Sampling and Analysis of SWSA 6 trench leachates and groundwaters. ORNL/TM-10813. Oak Ridge National Laboratory, Oak Ridge, Tenn.

Sppalding, B. P., G. K. Jacobs, and E. C. Davis. 1989. Demonstrations of technology for remediation and closure of Oak Ridge National Laboratory Waste Disposal Sites. ORNL/TM11286. Oak Ridge National Laboratory, Oak Ridge, Tenn.

Verschueren, K. 1983. Handbook of Environmental Data on Organic Chemicals. 2nd Ed. VanNostrand Reinhold Publishers, New York. 


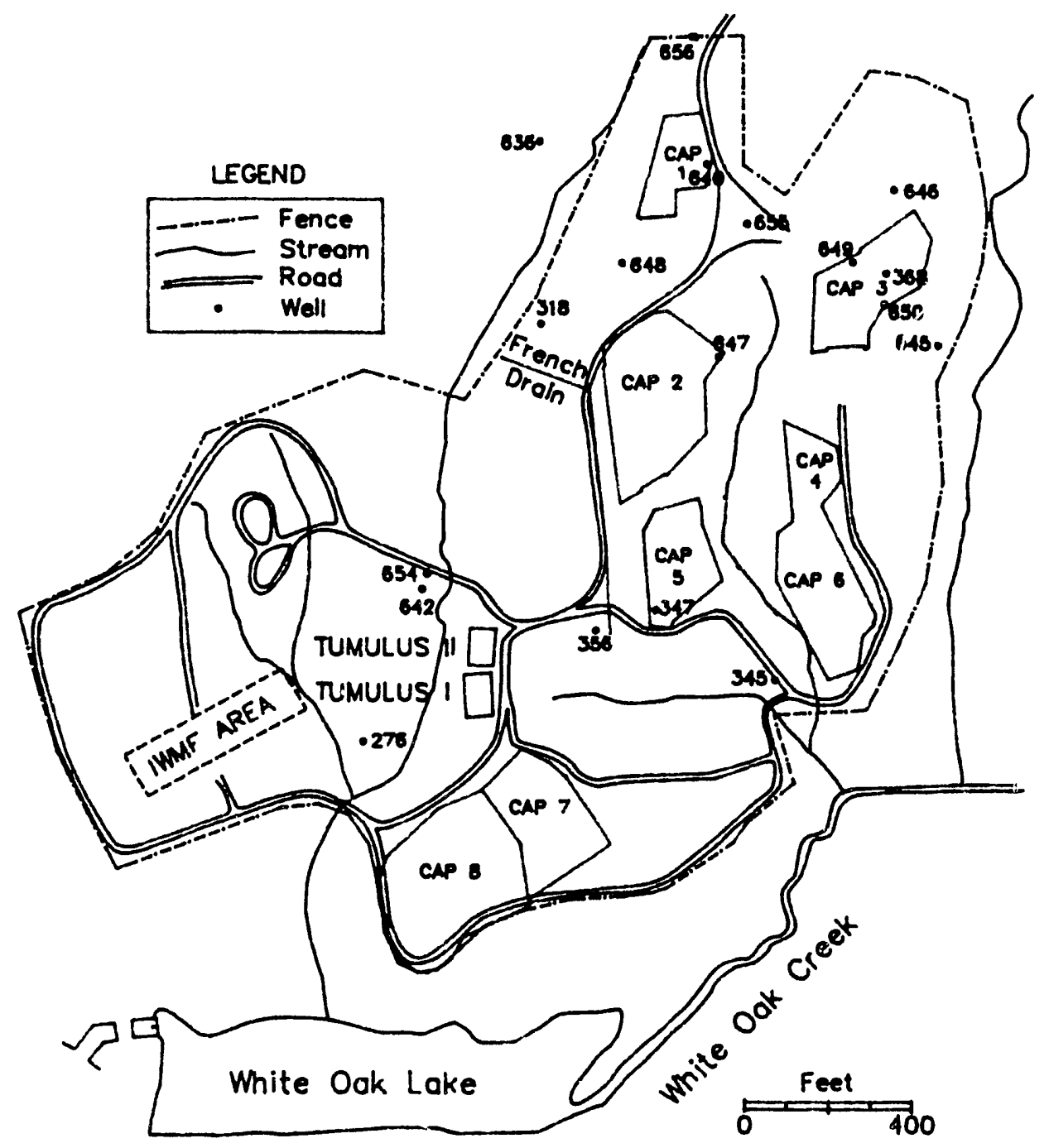

Fig. 1. Solid Waste Storage Area 6 showing interim corrective measures capped areas, French drain, monitoring wells outside burial trenches, and major low-level waste disposal areas. 


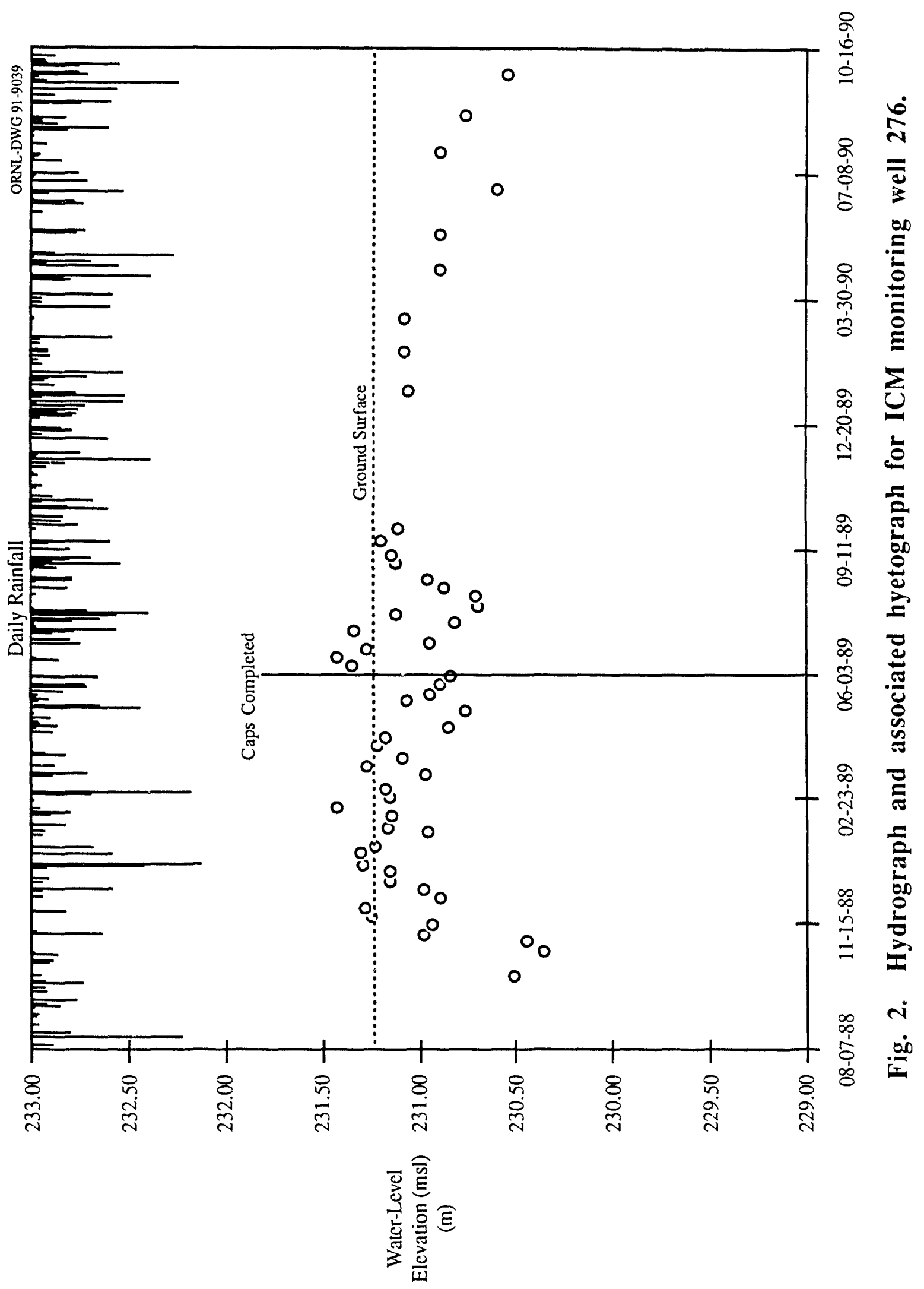




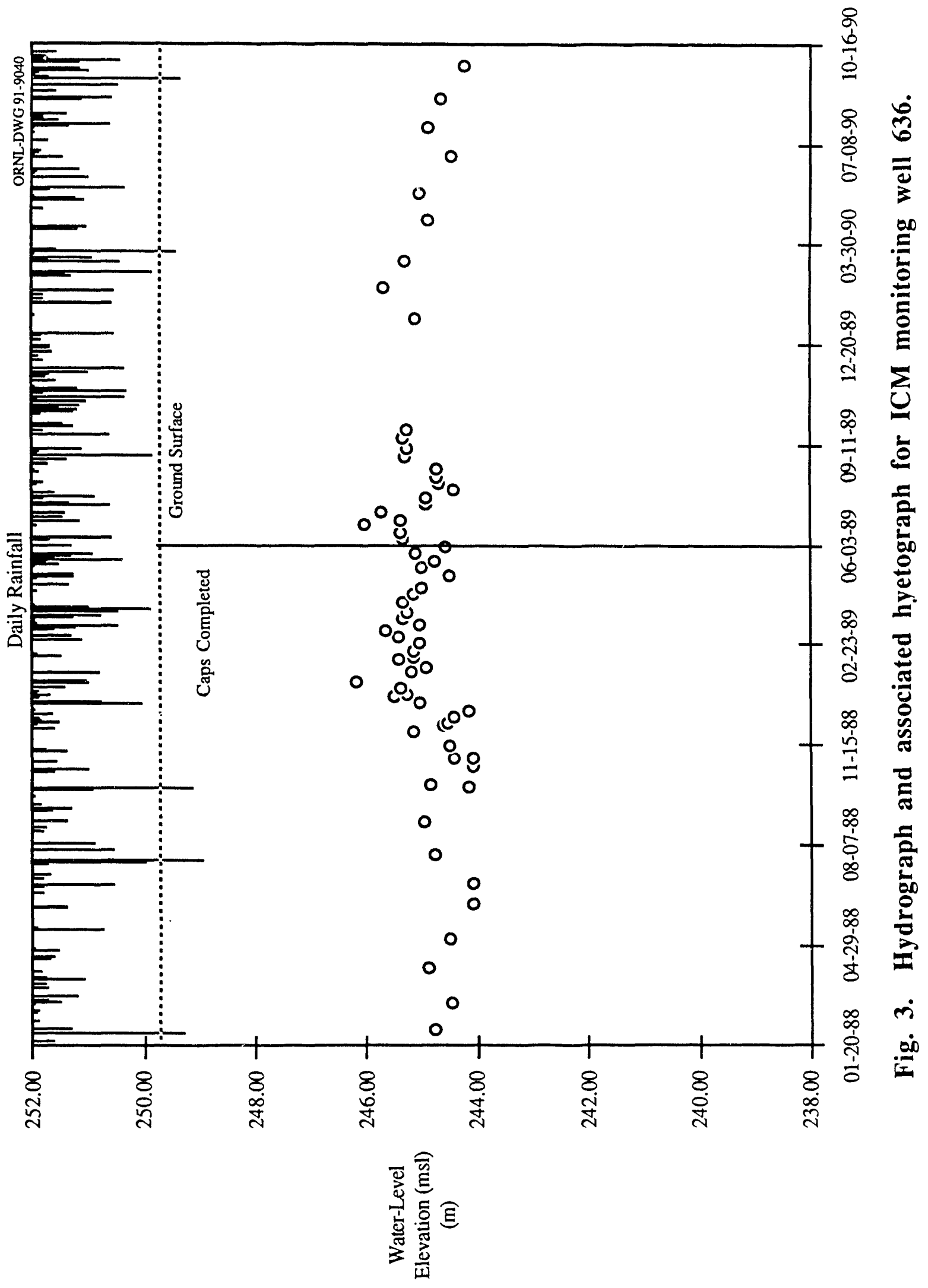




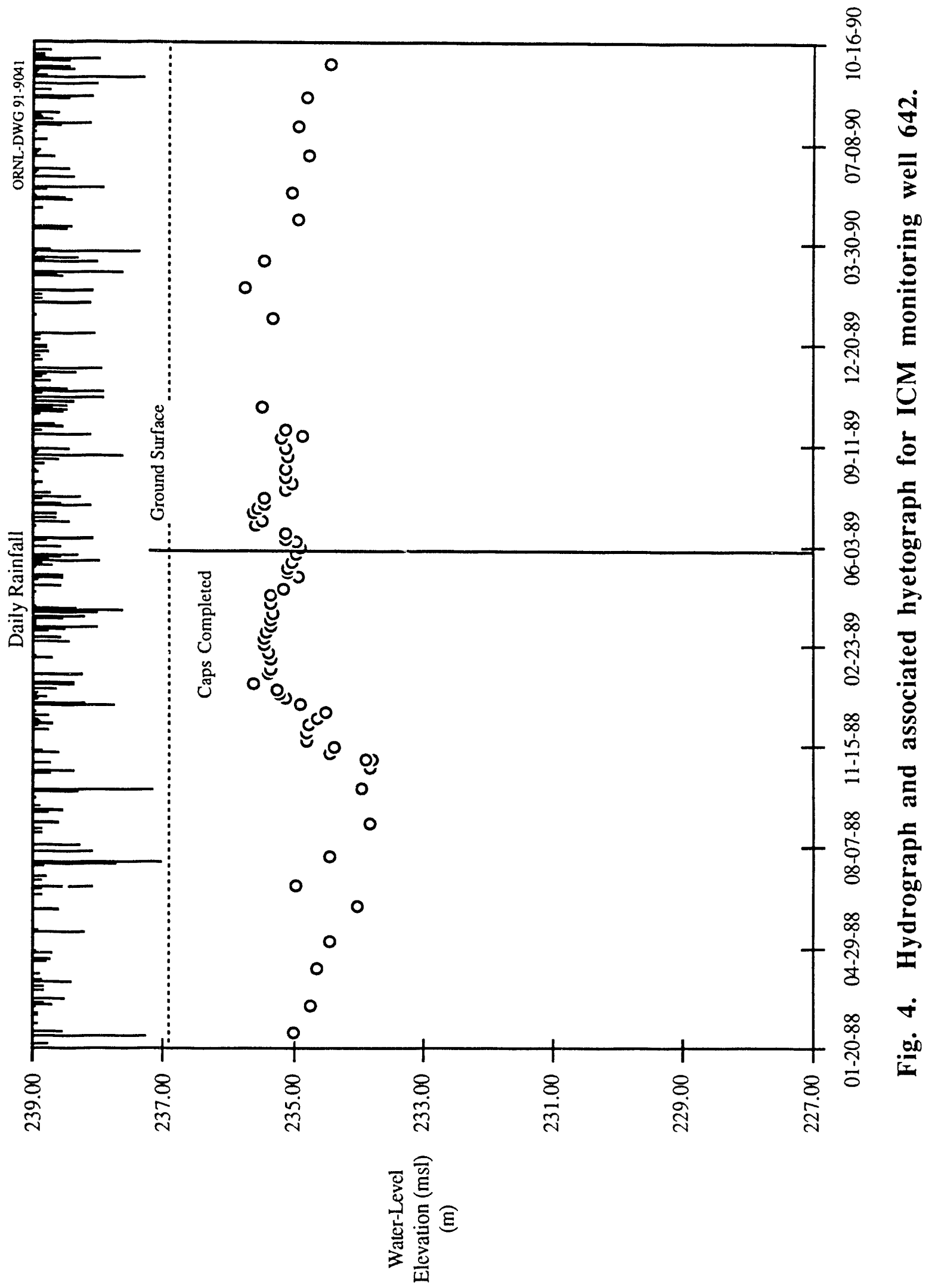




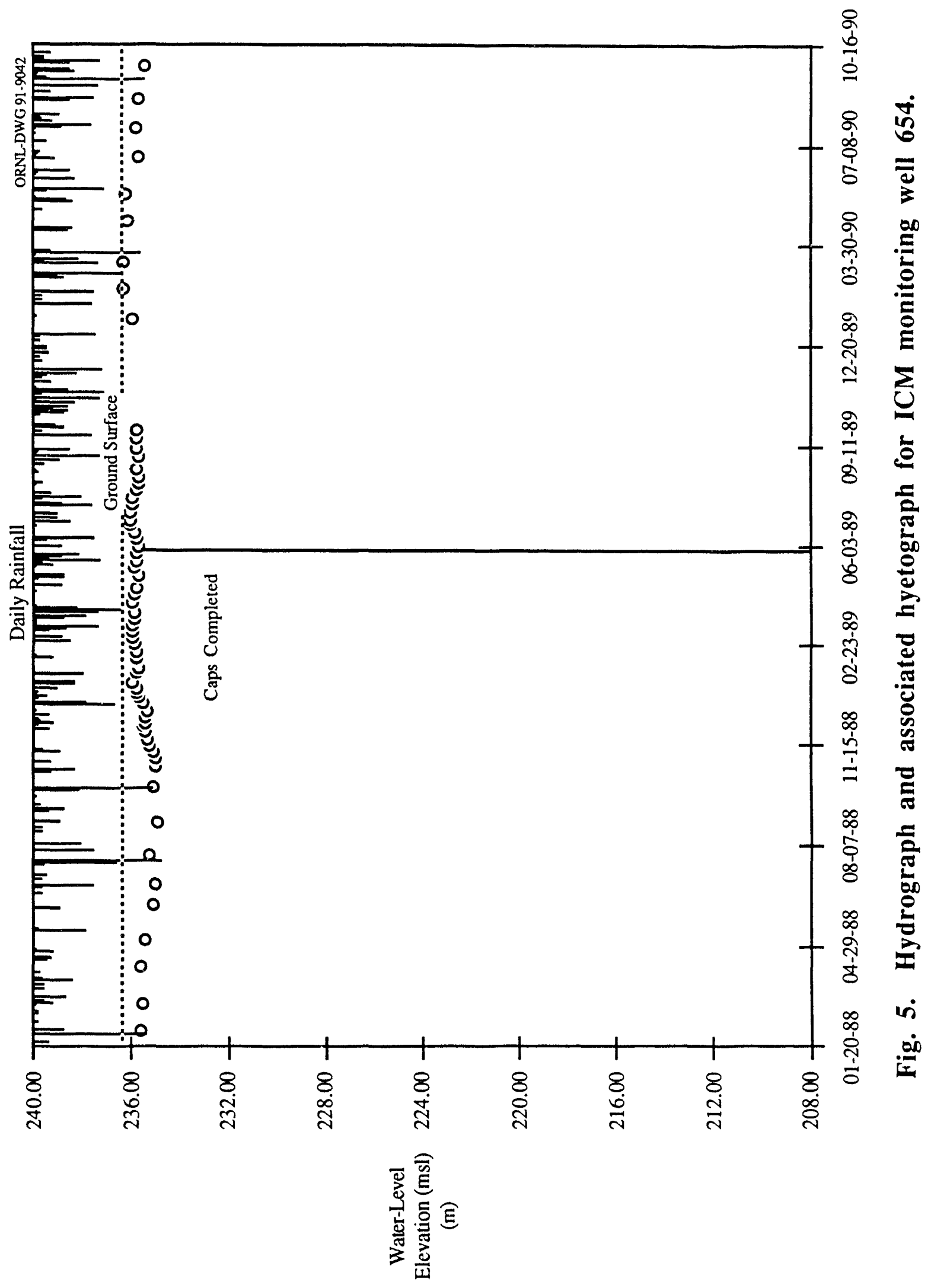




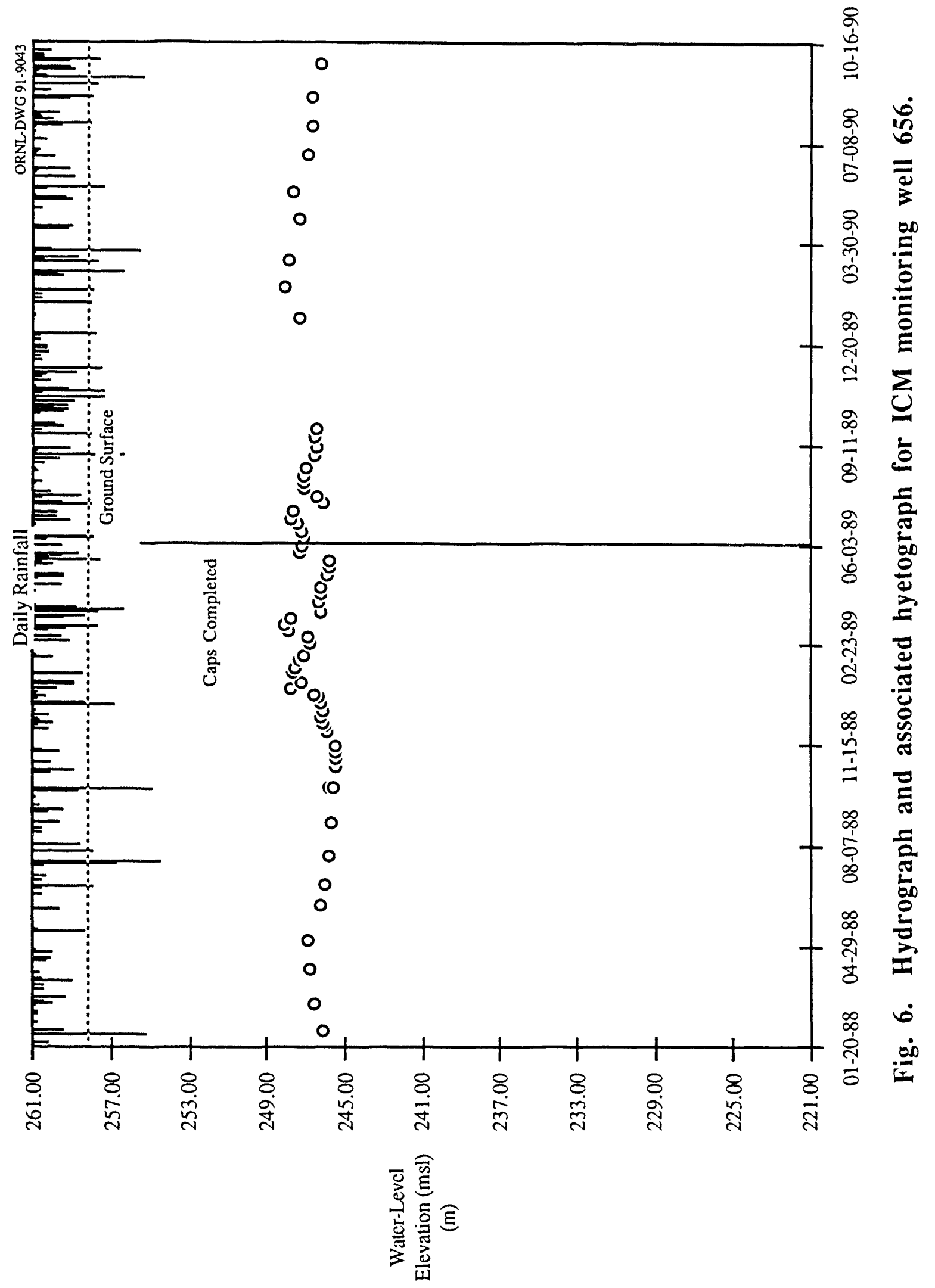




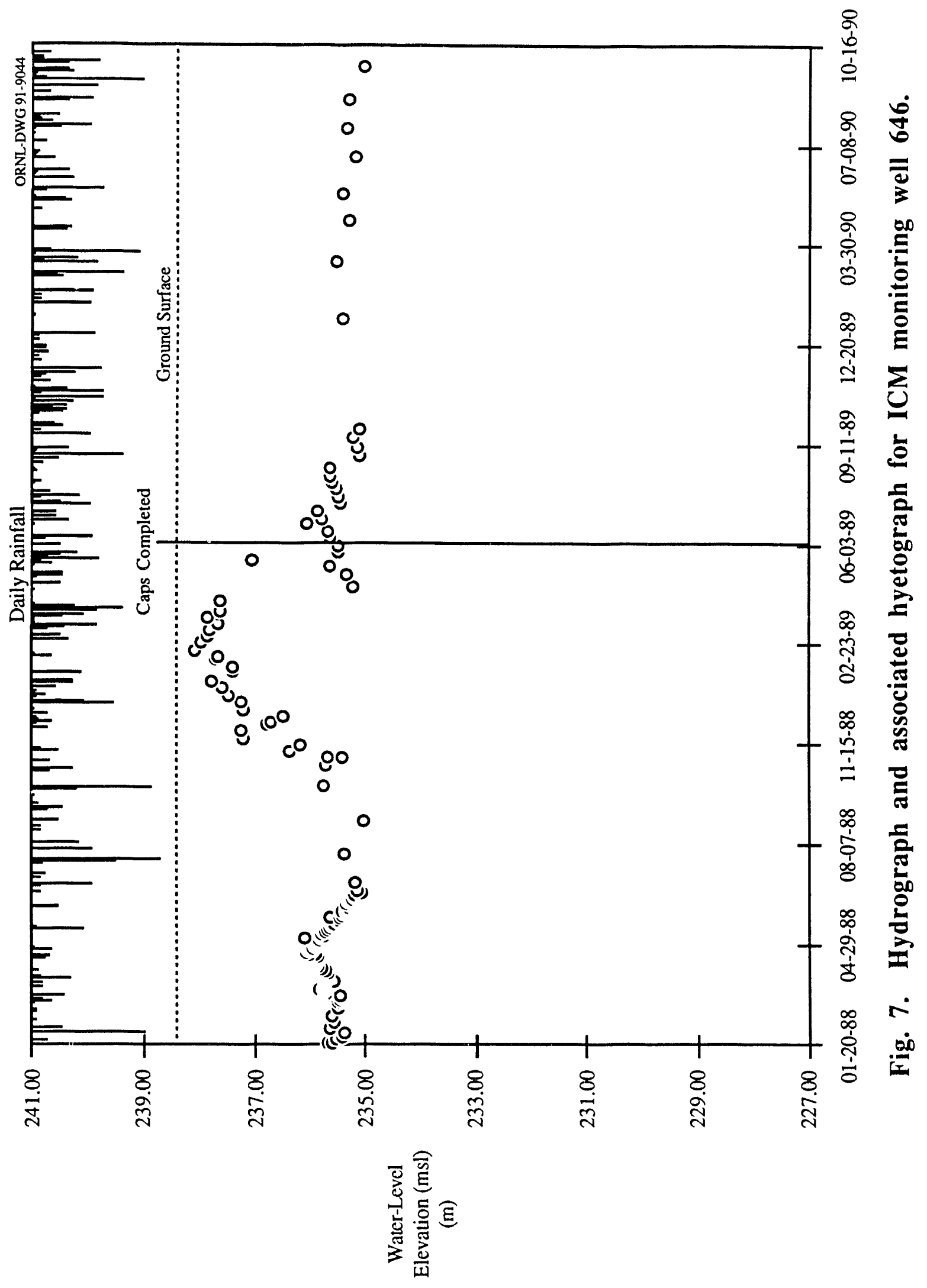




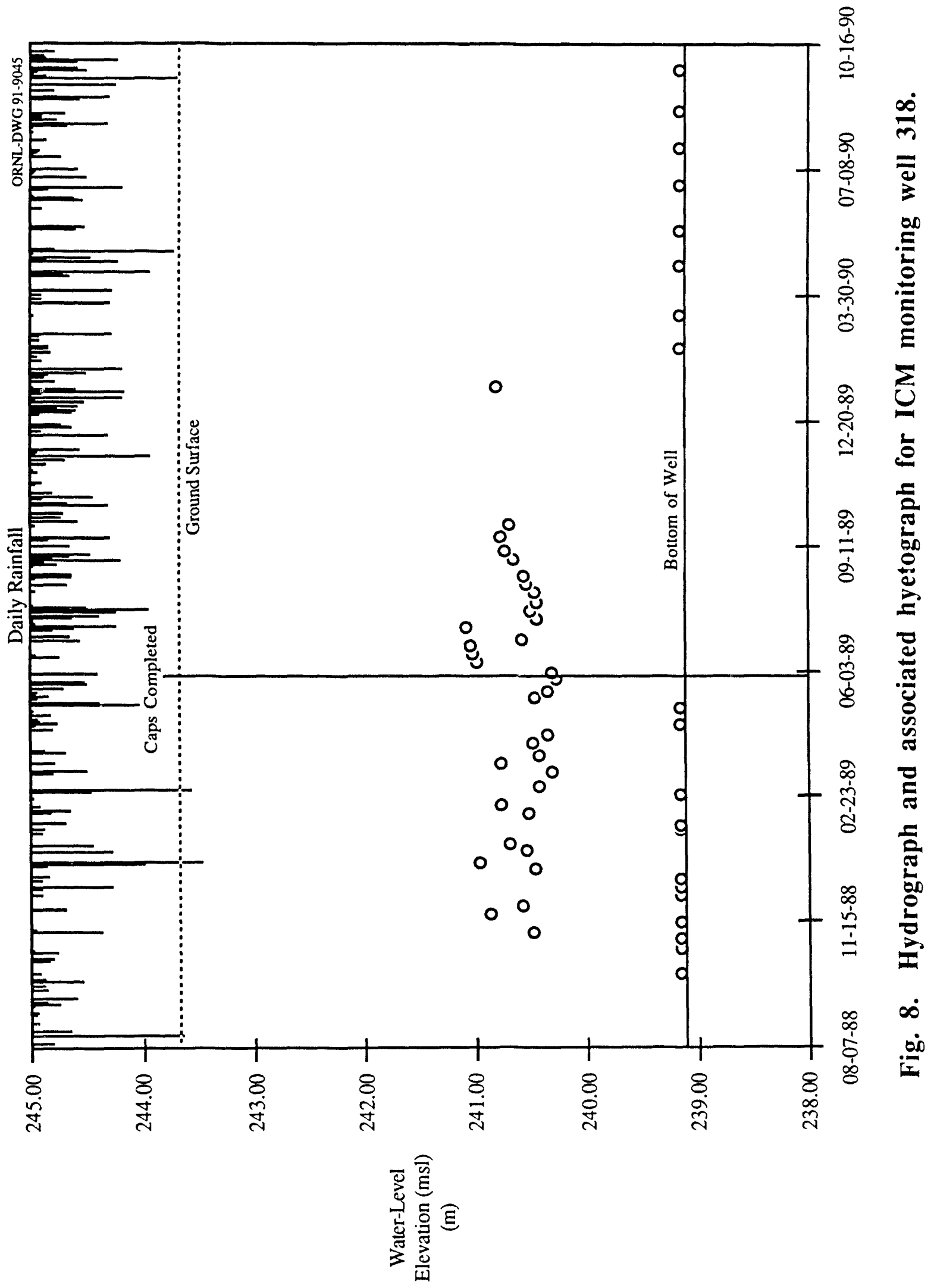




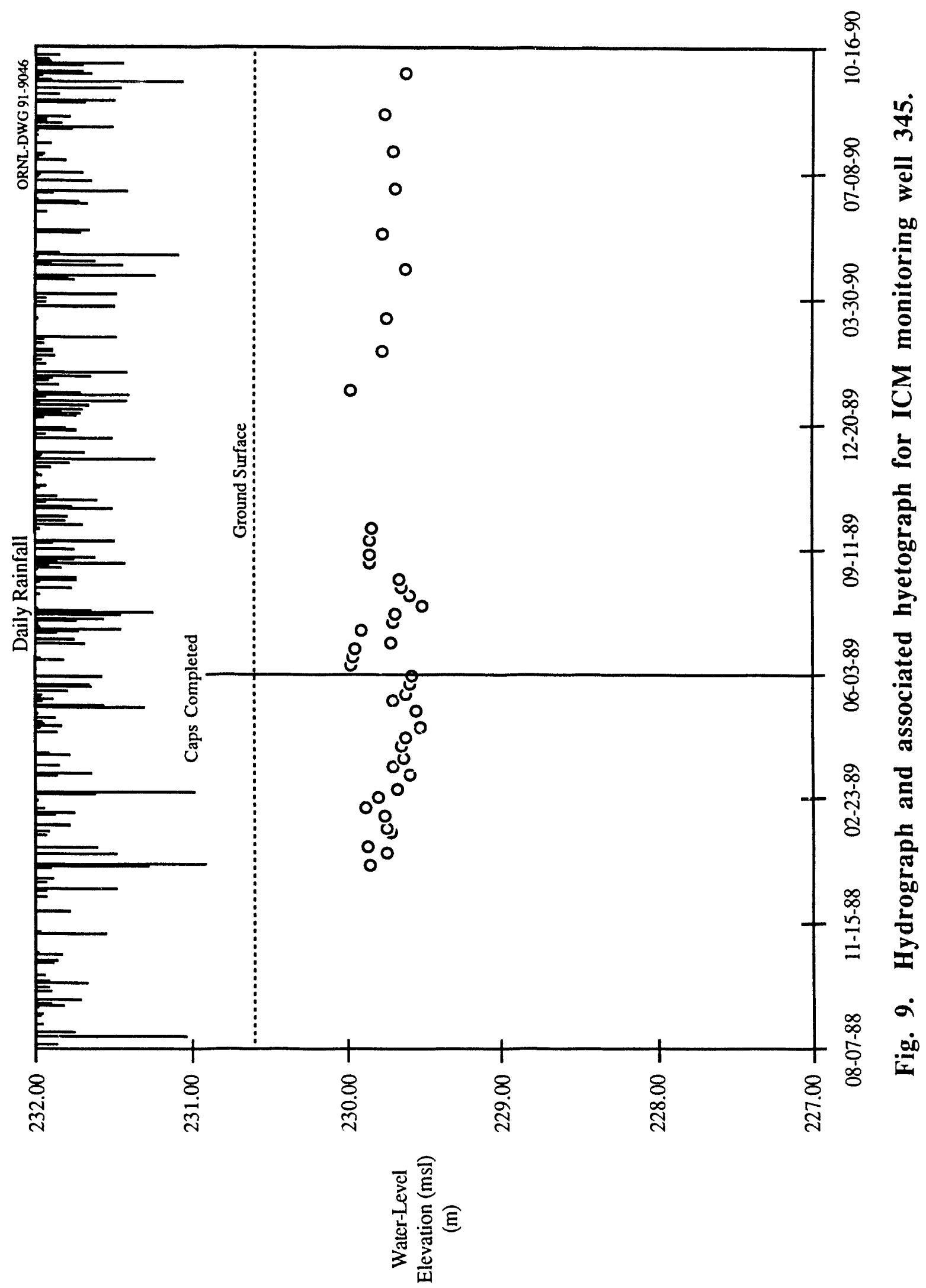




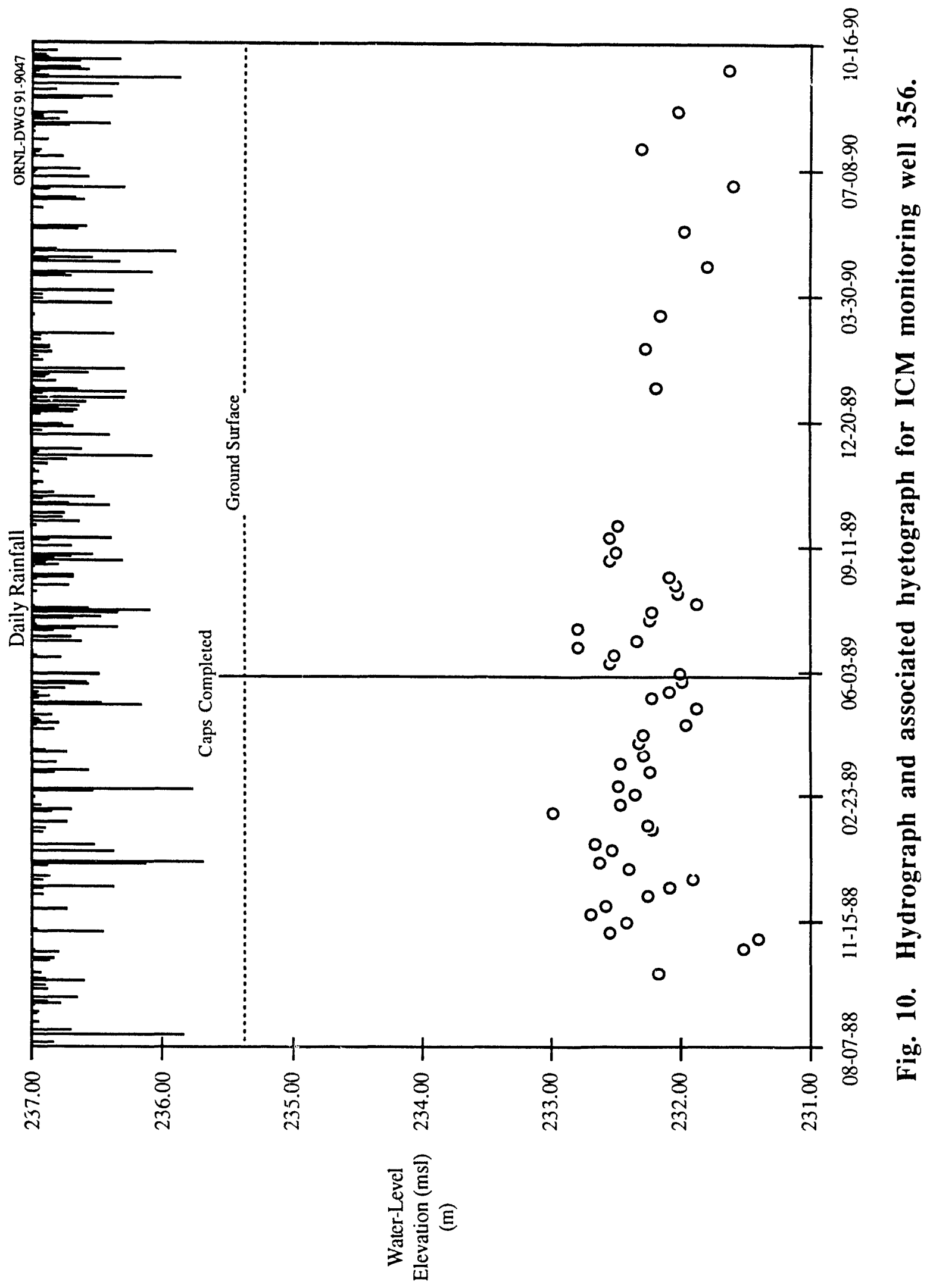




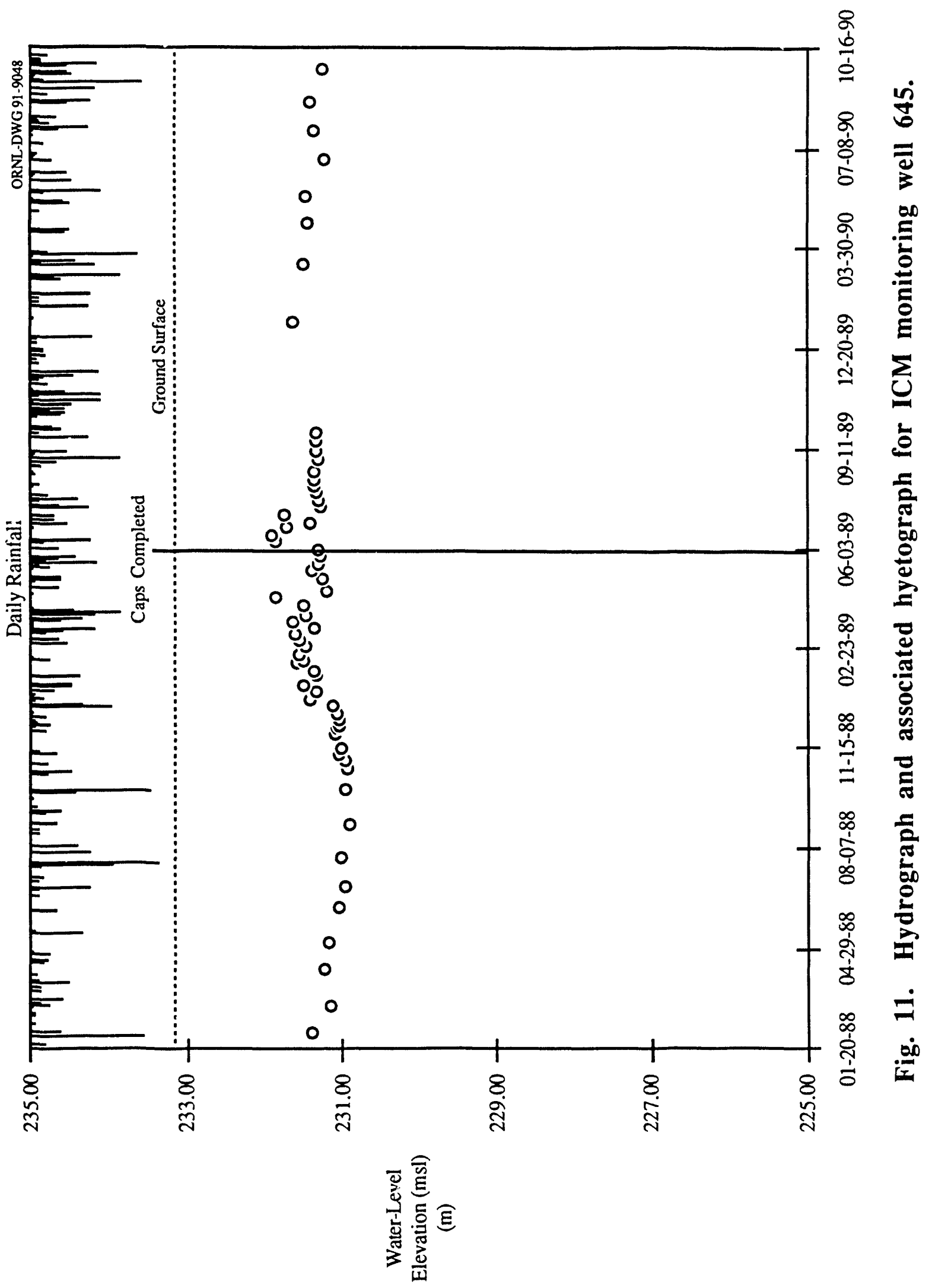




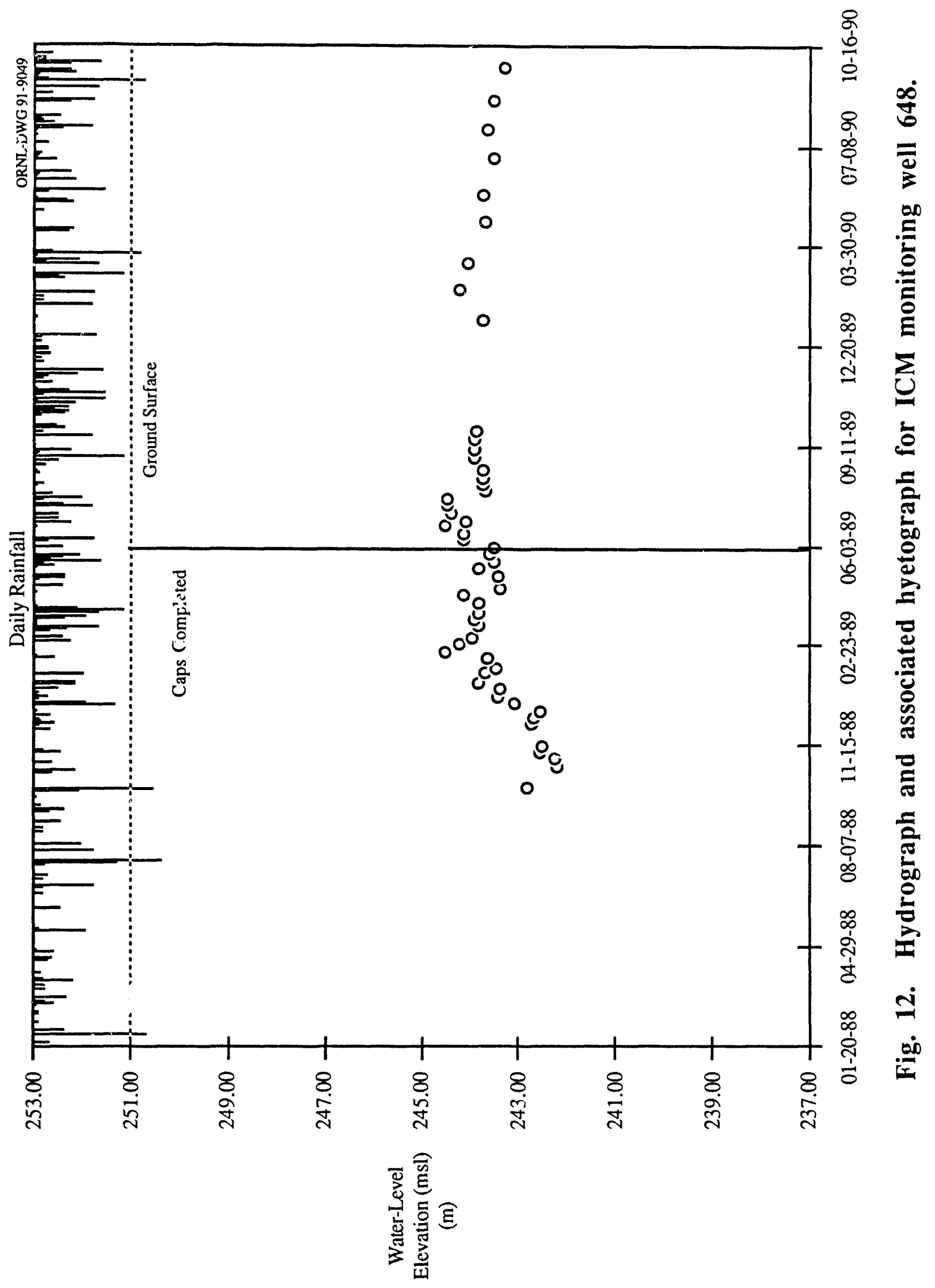




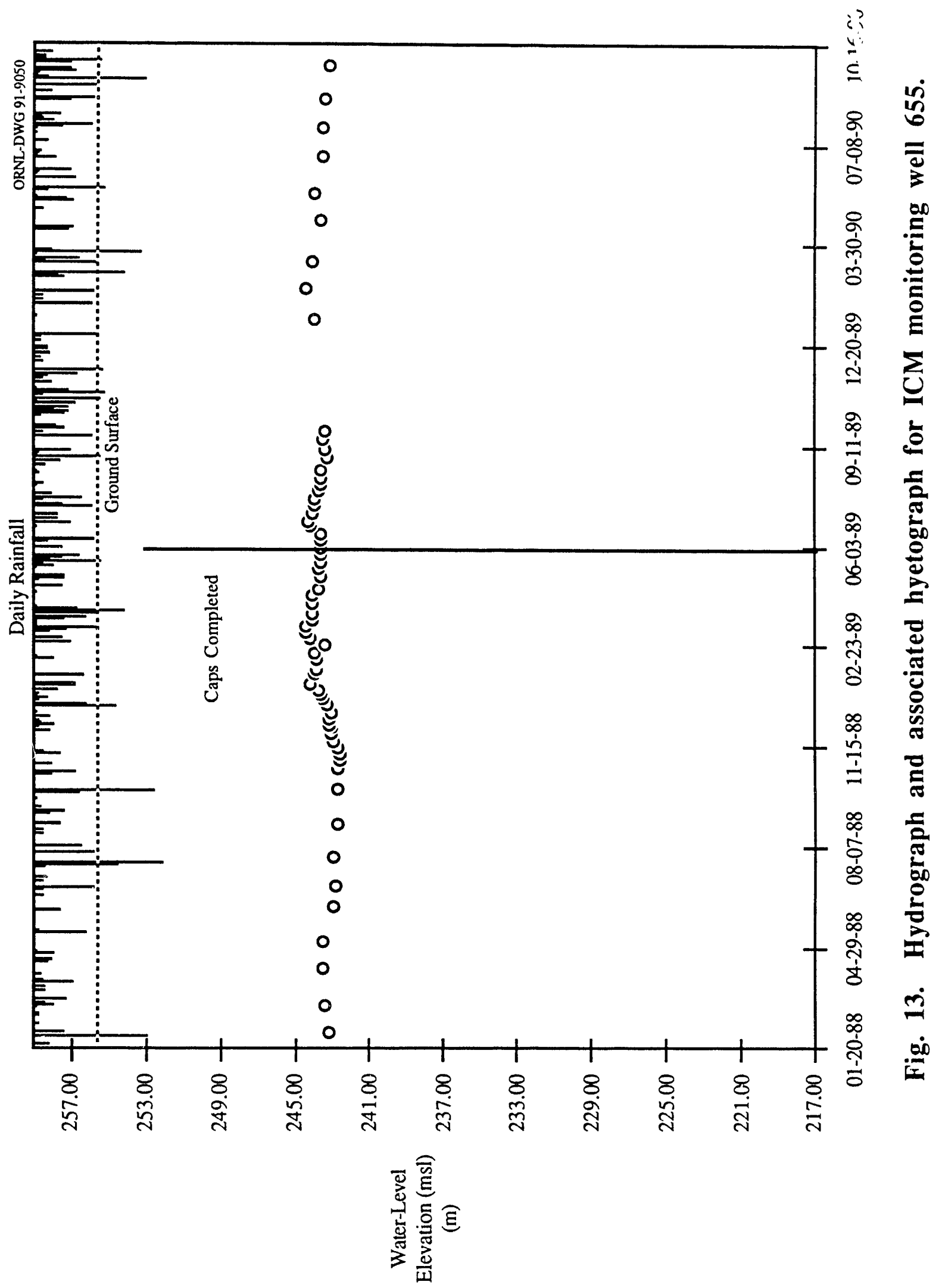




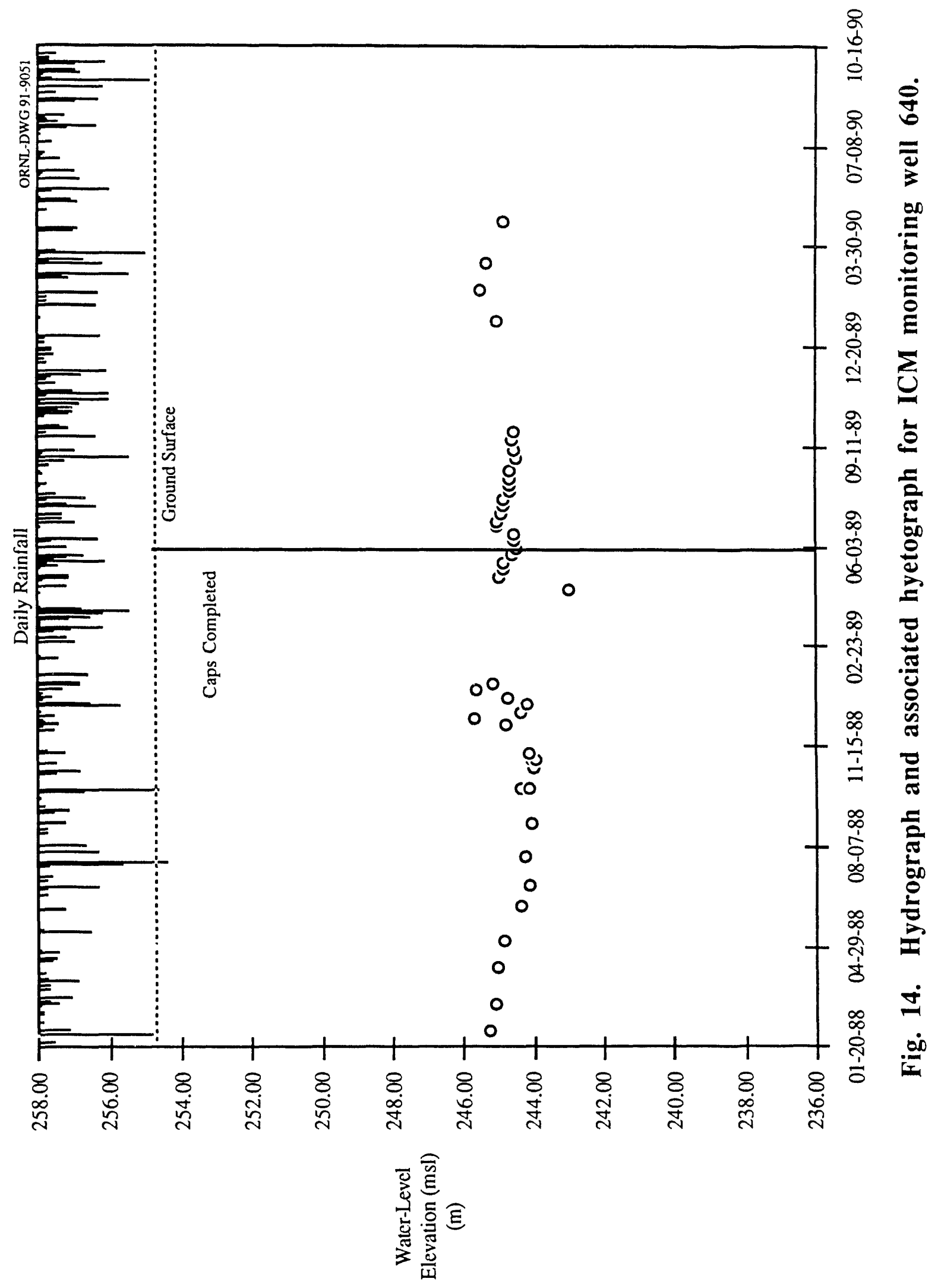




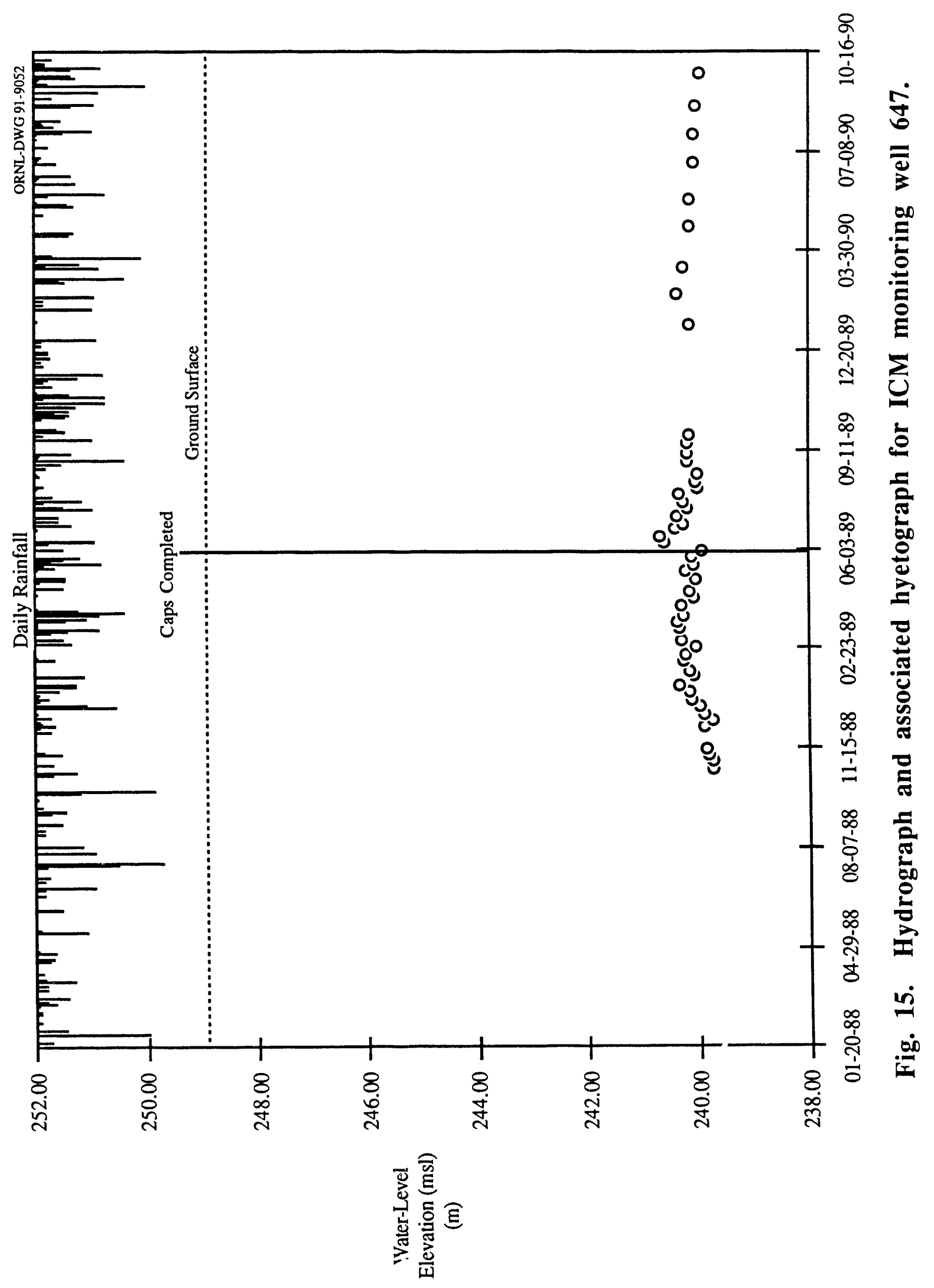




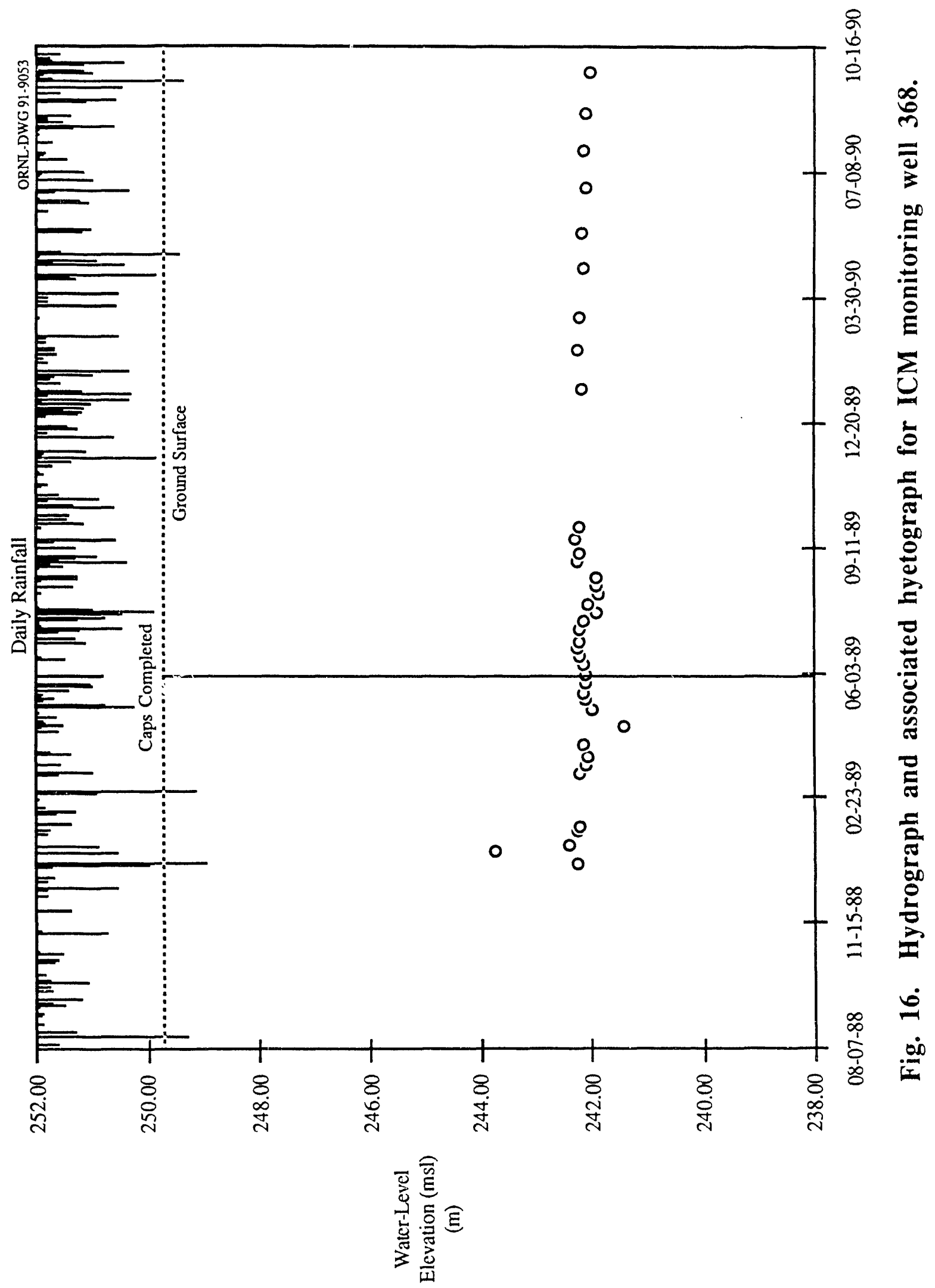




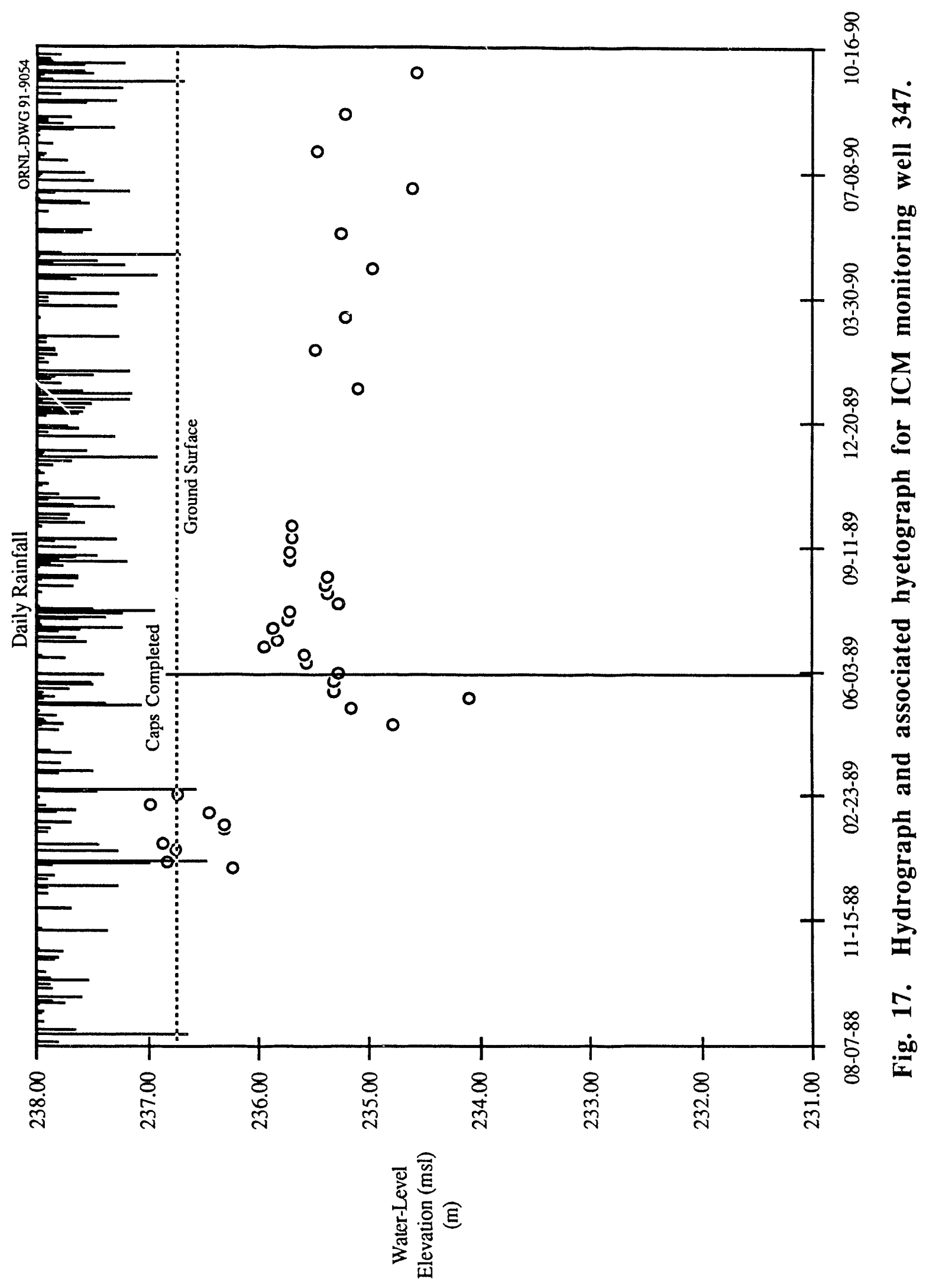


ORNL-DWG 91-9055

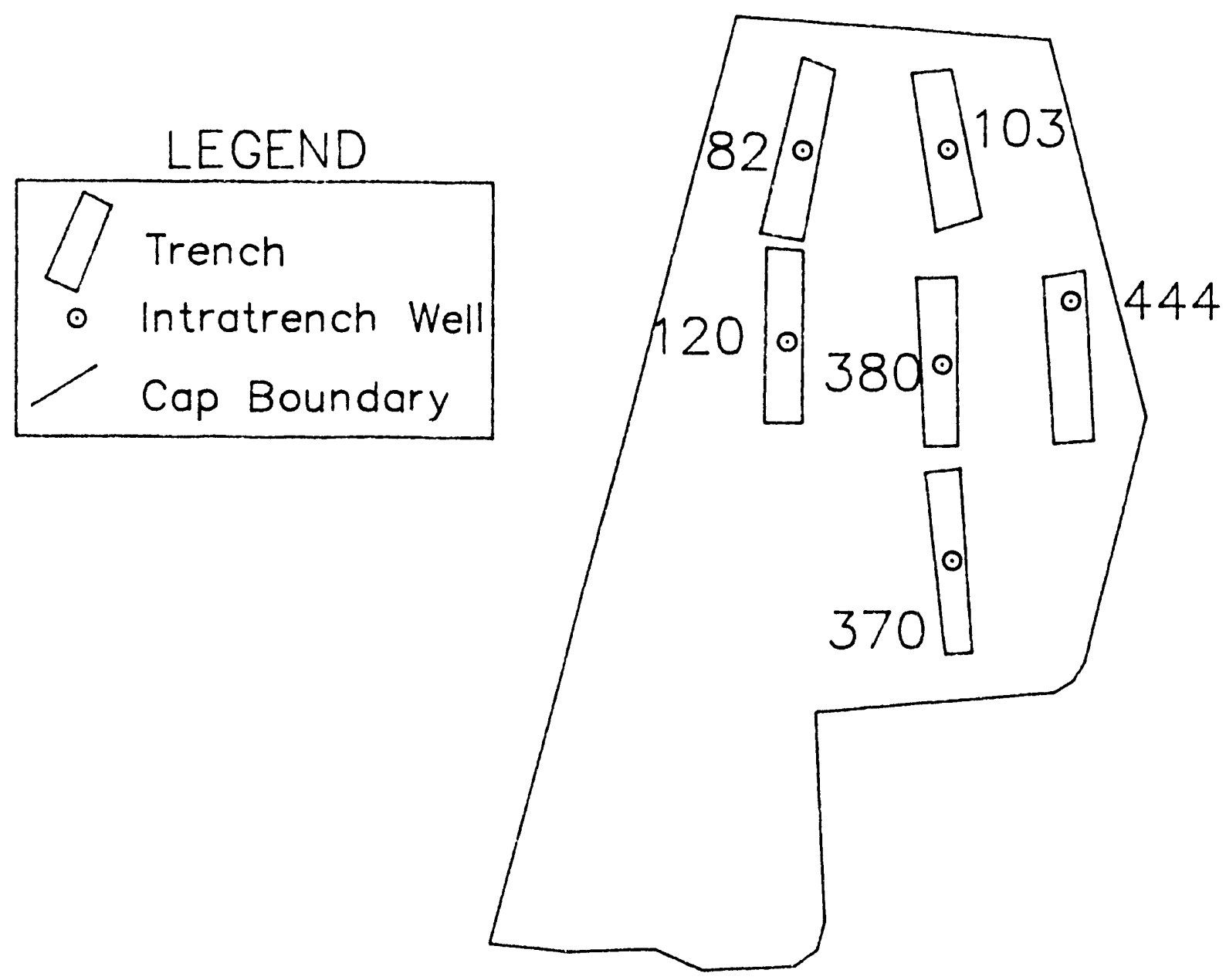

Fig. 18. Burial trench and well locations within cap area 1. 


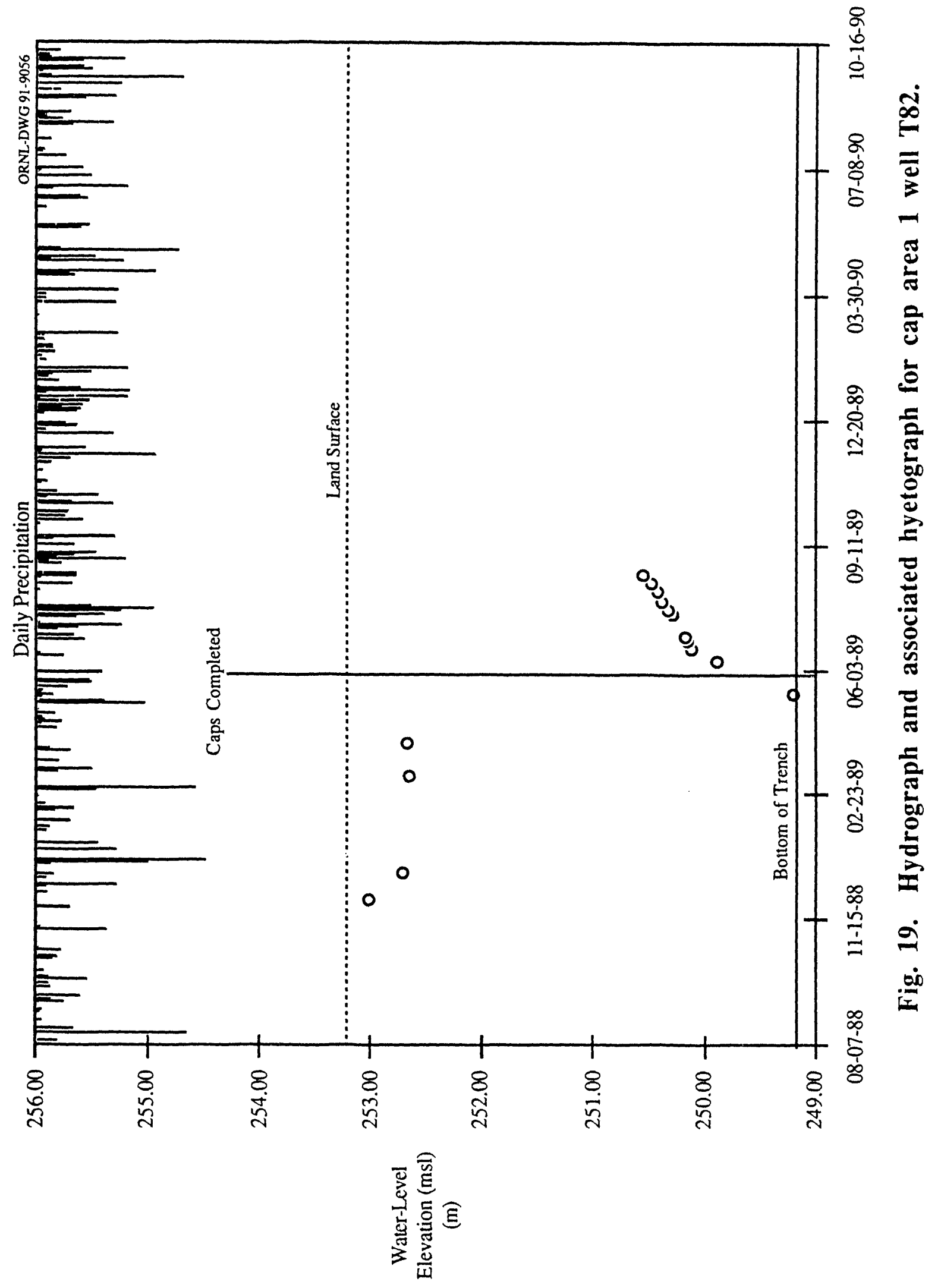




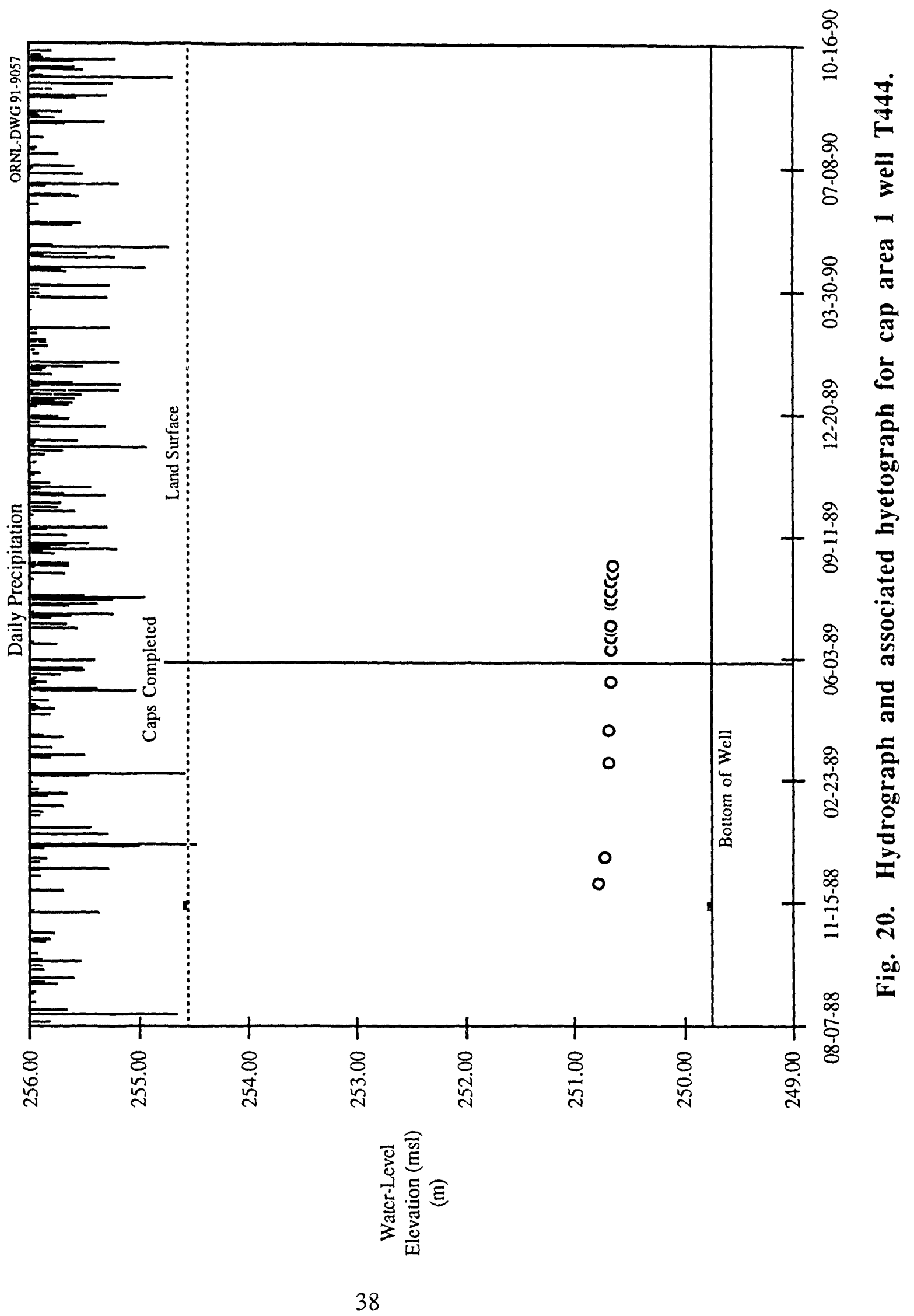




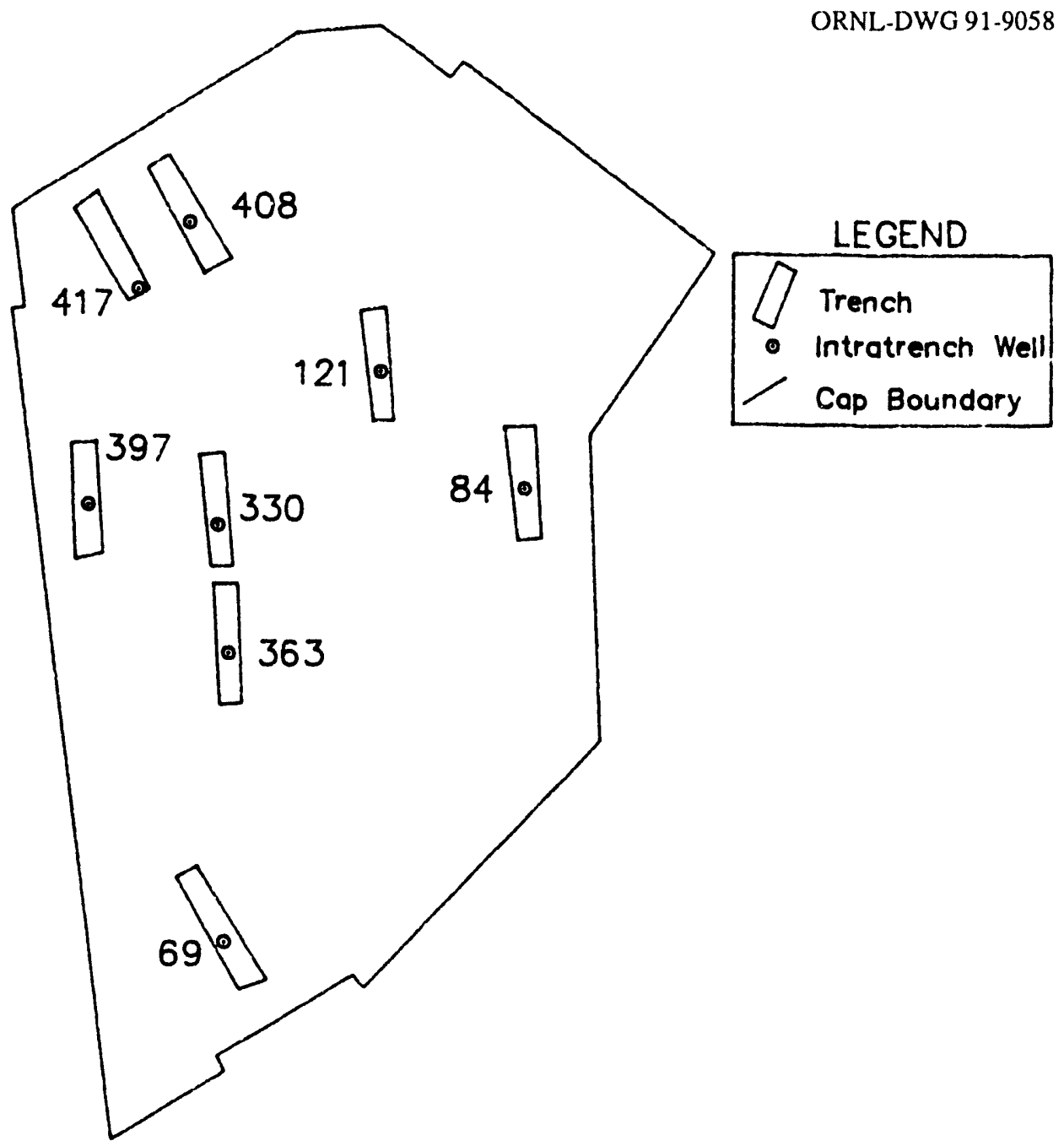

Fig. 21. Burial trench and well locations within cap area 2. 


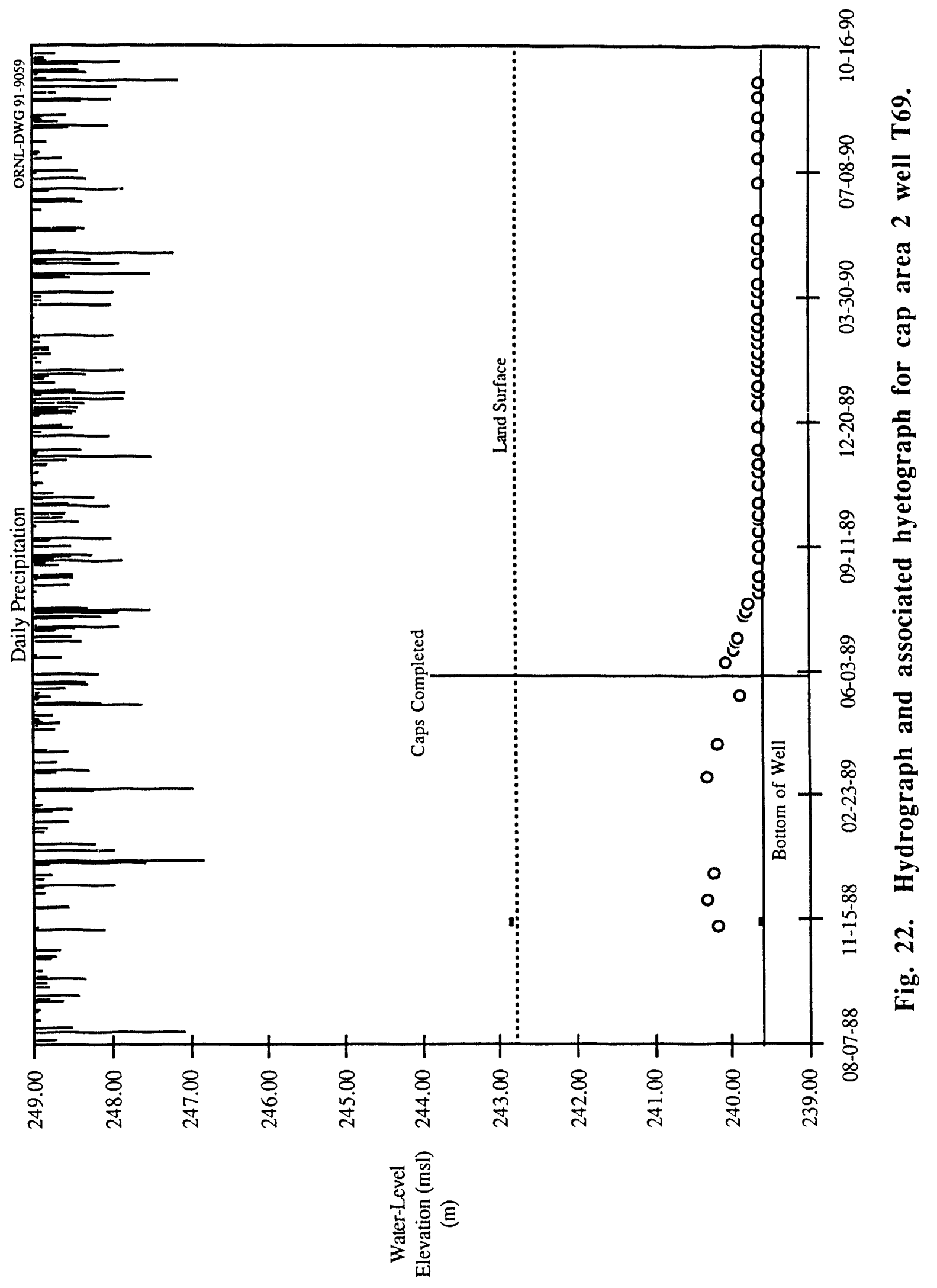




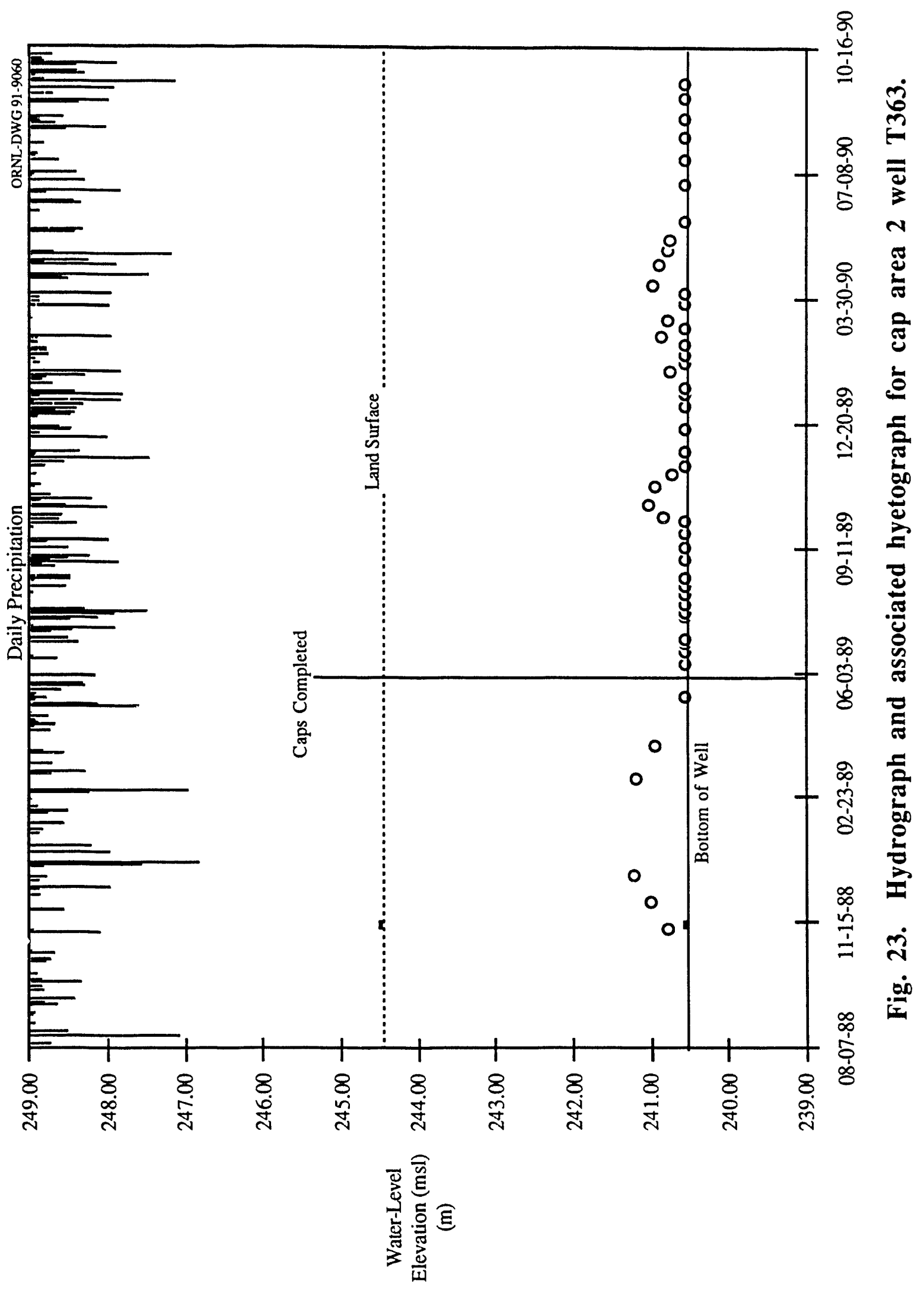




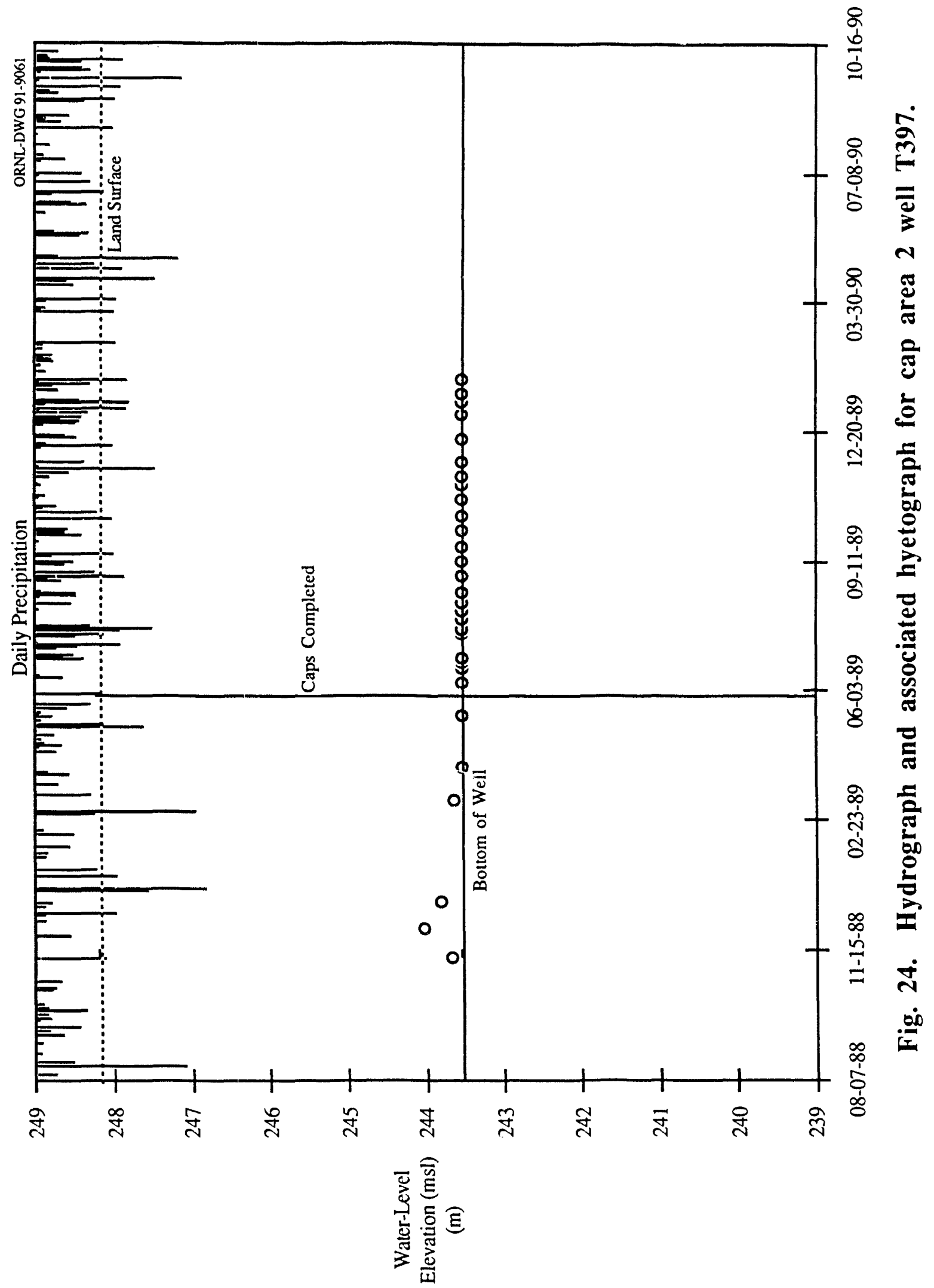




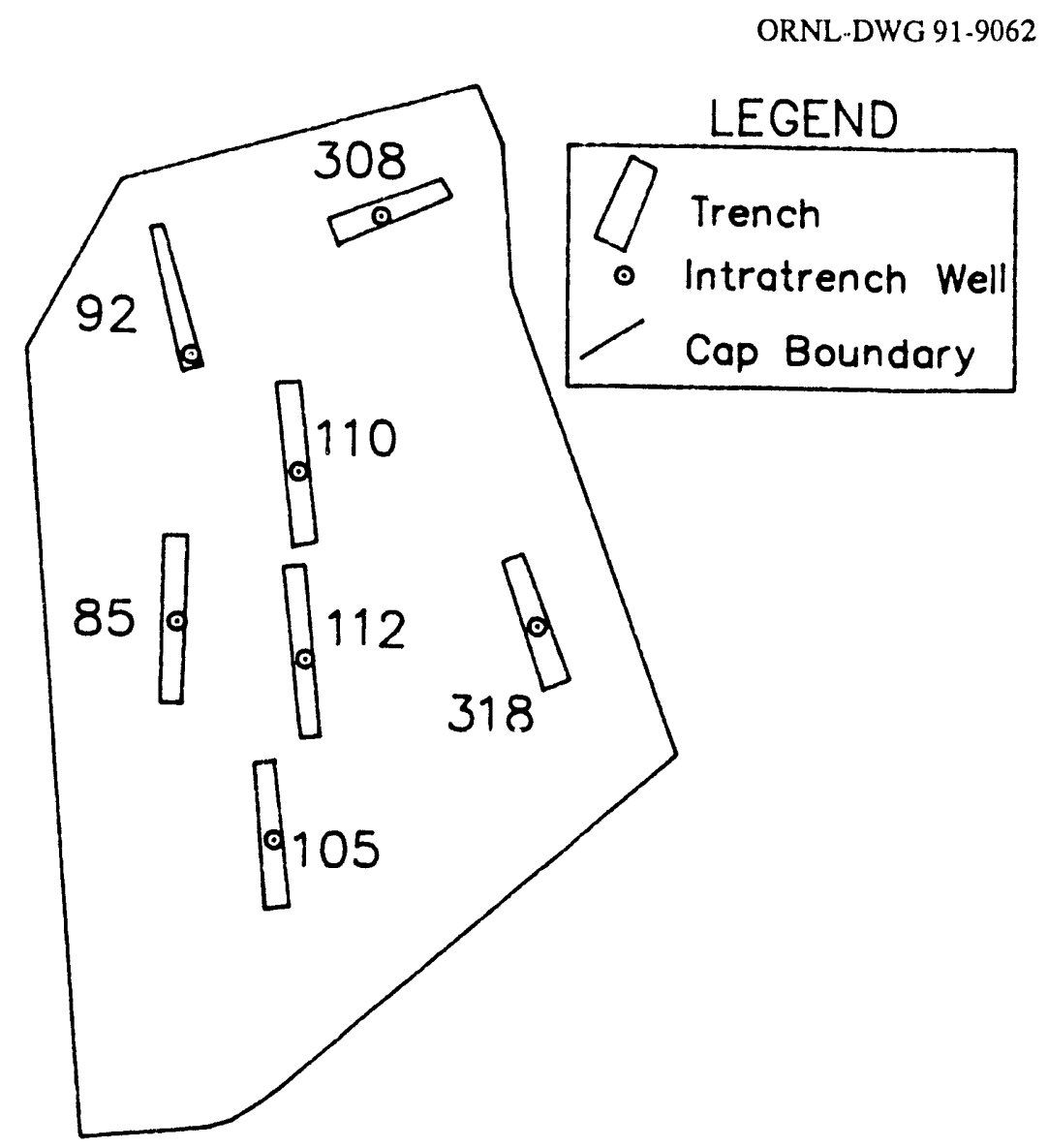

Fig. 25. Burial trench and well locations within cap area 5 . 


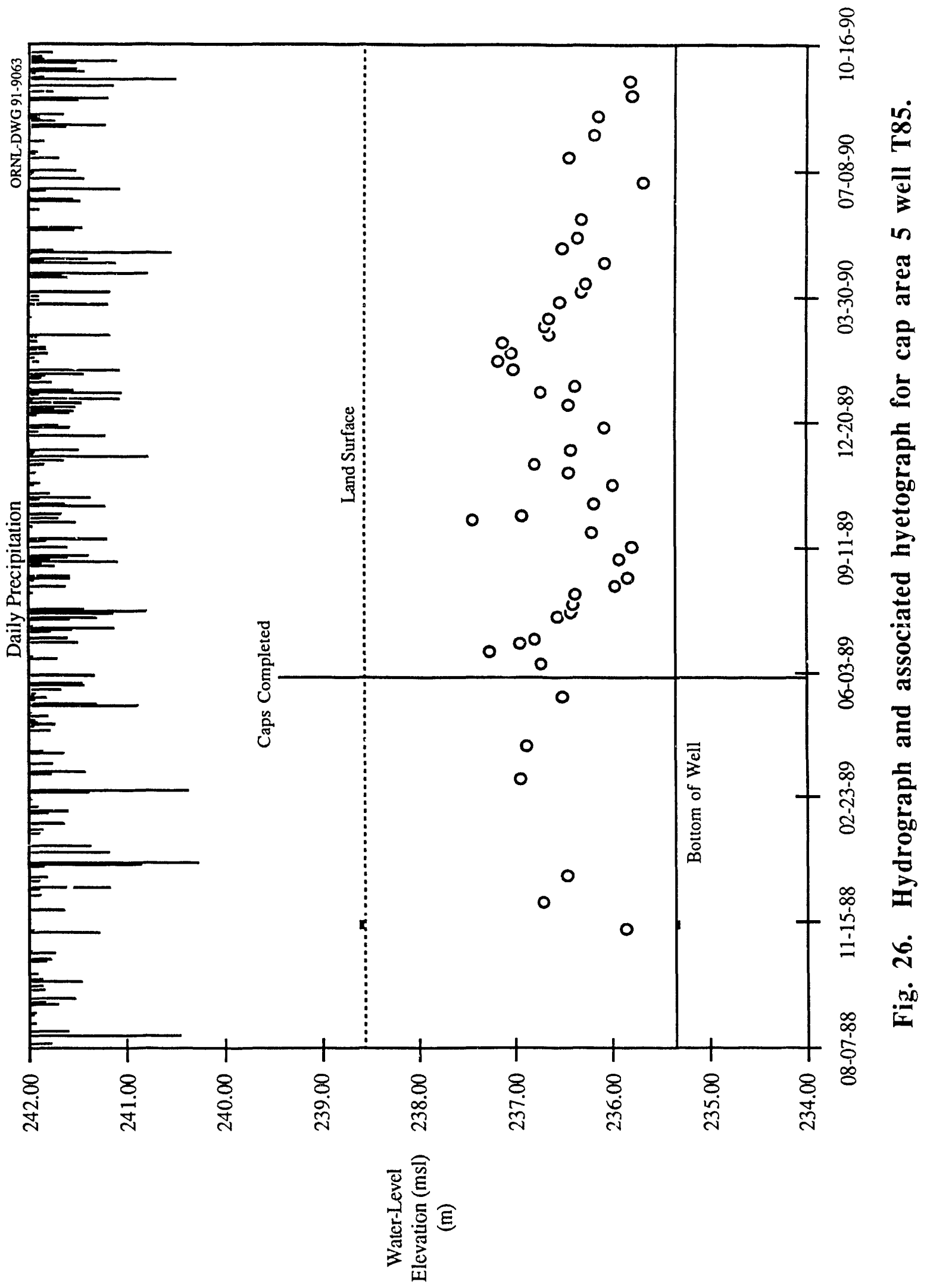




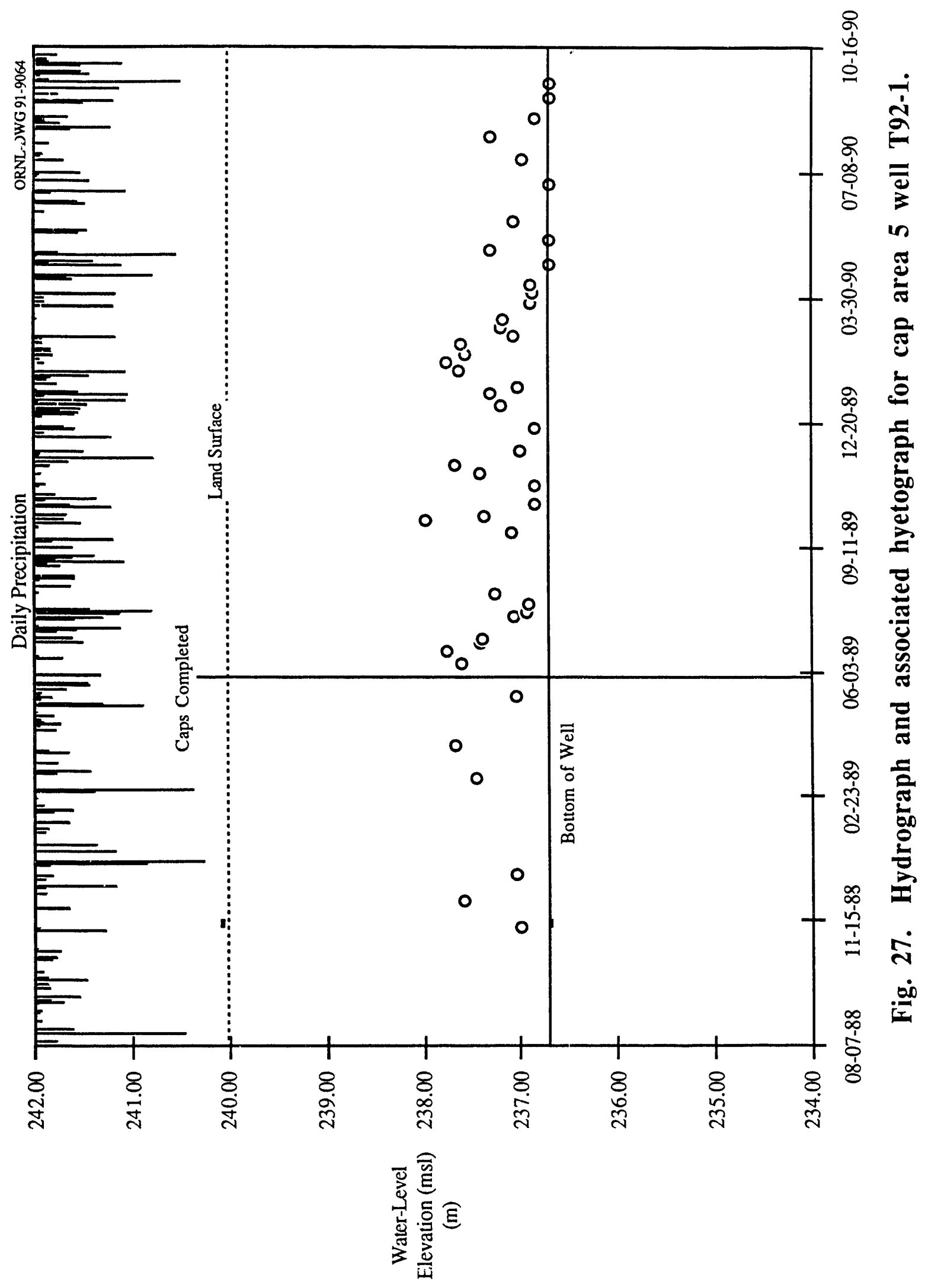




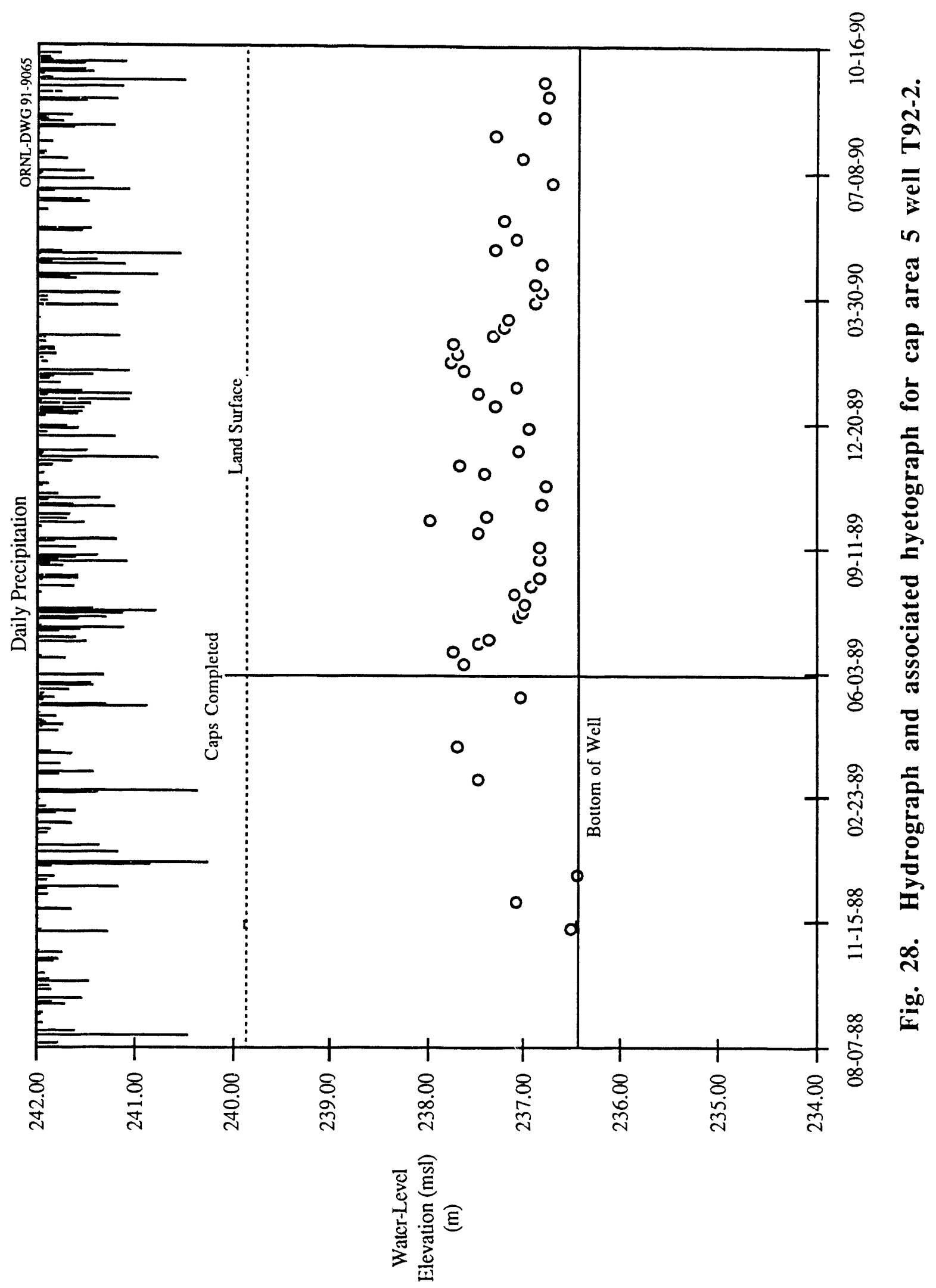




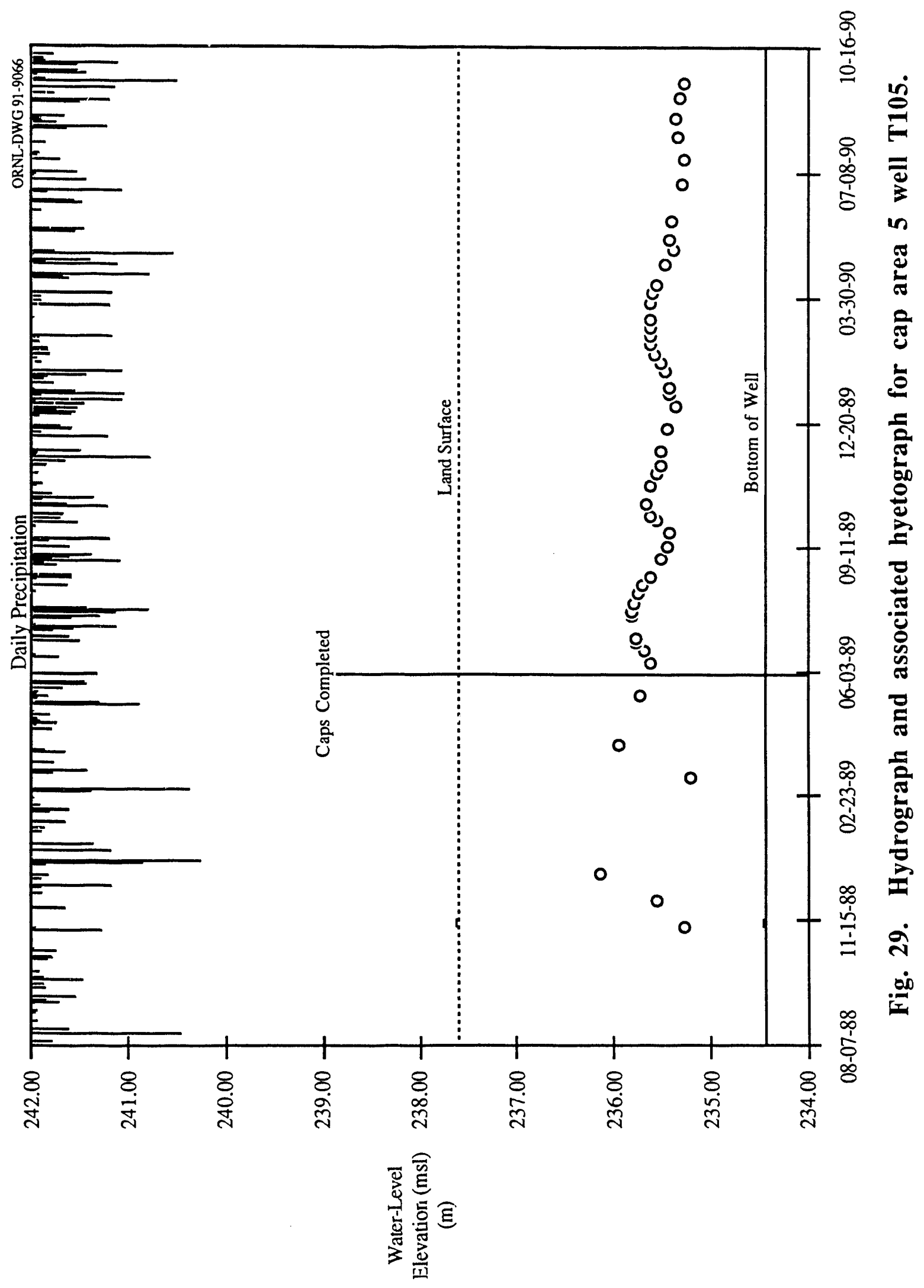




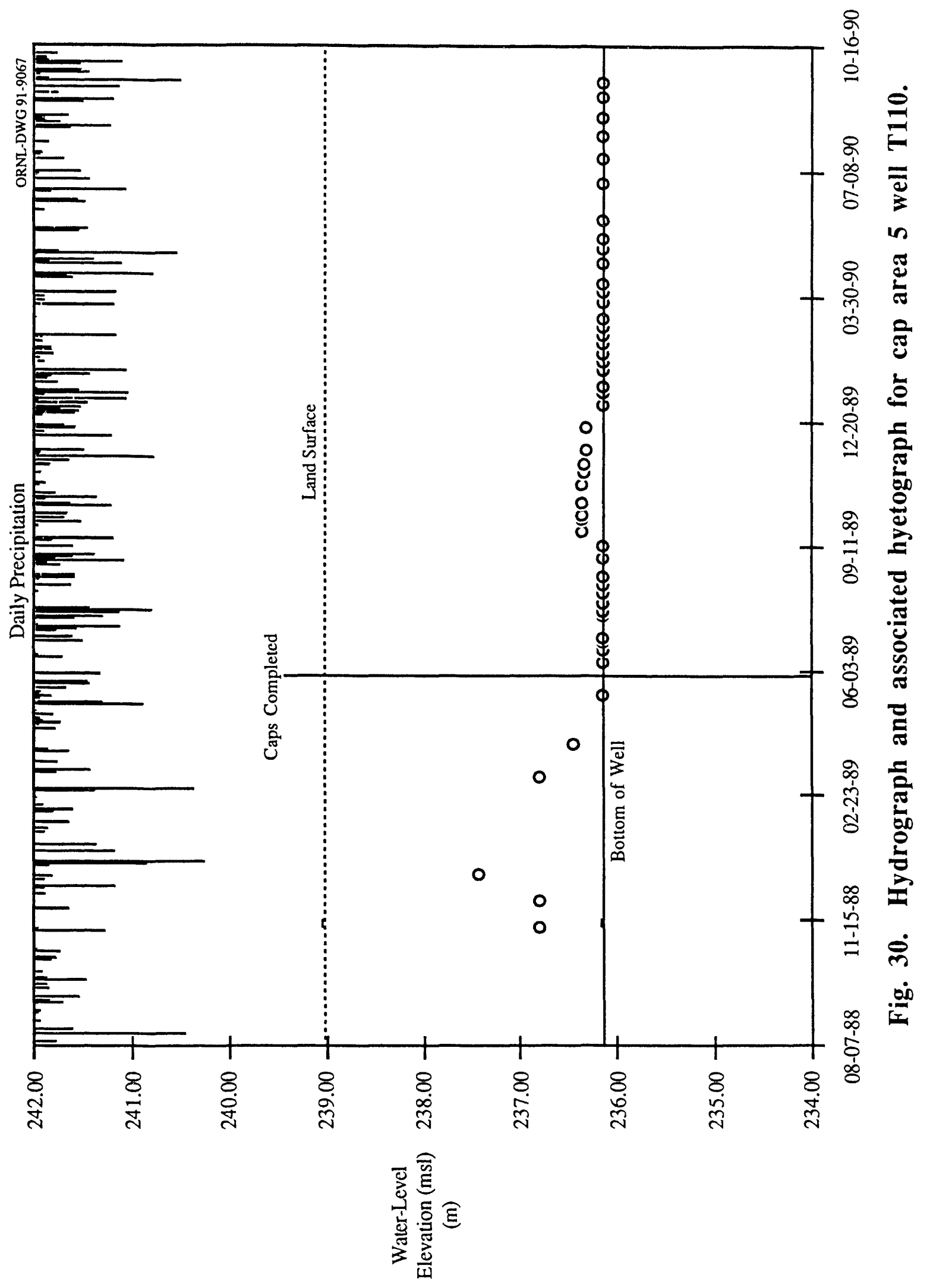




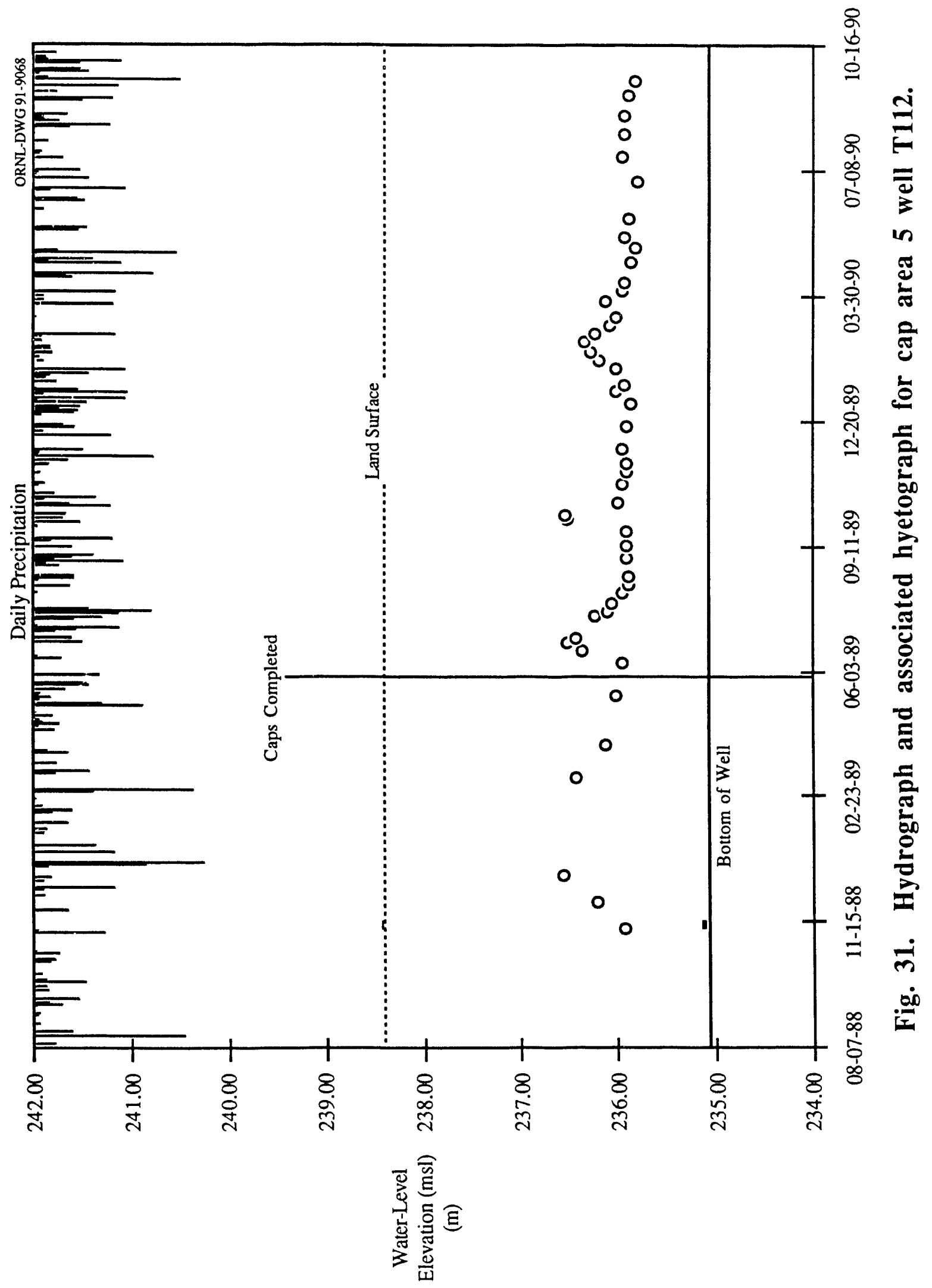




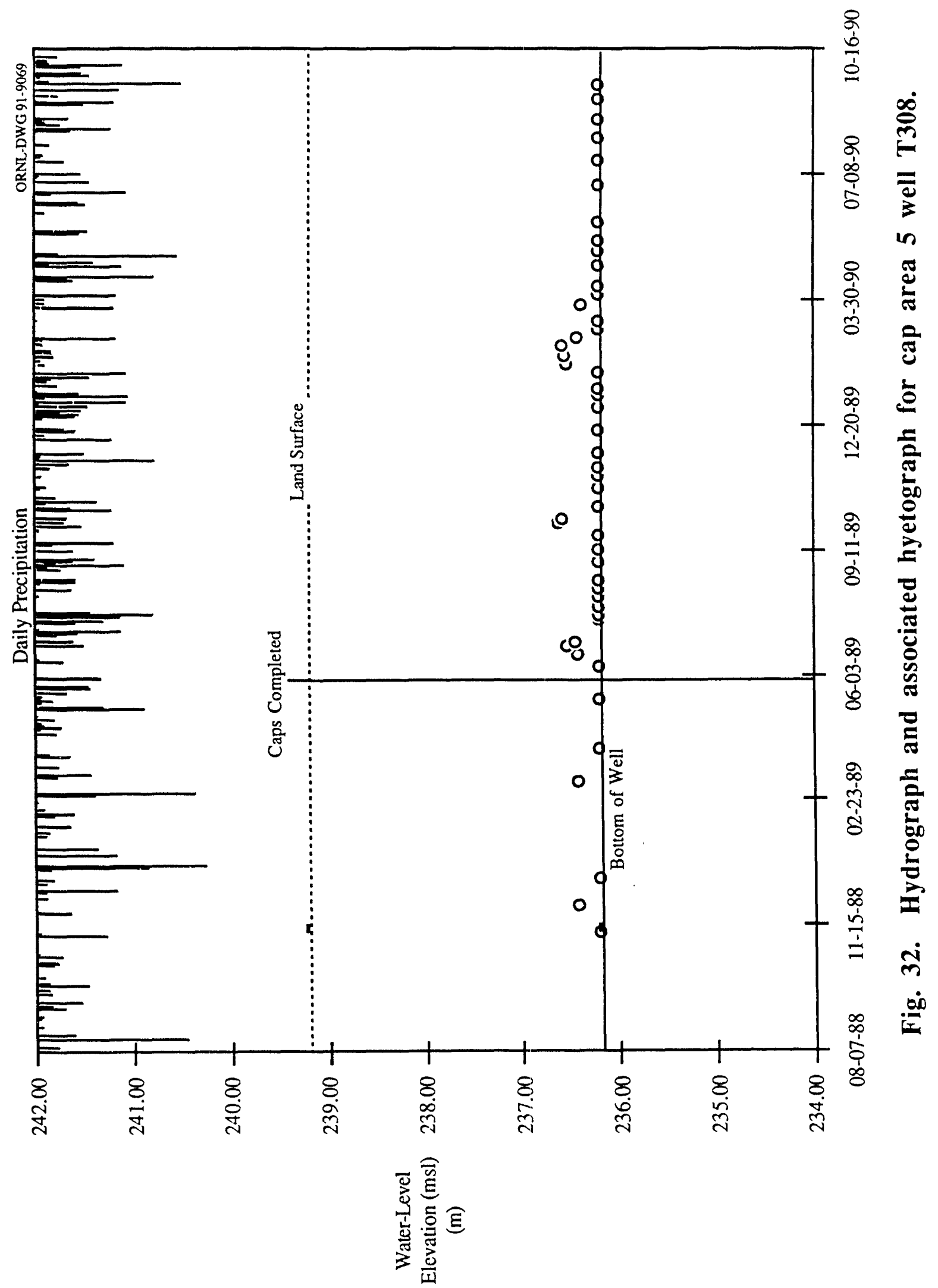




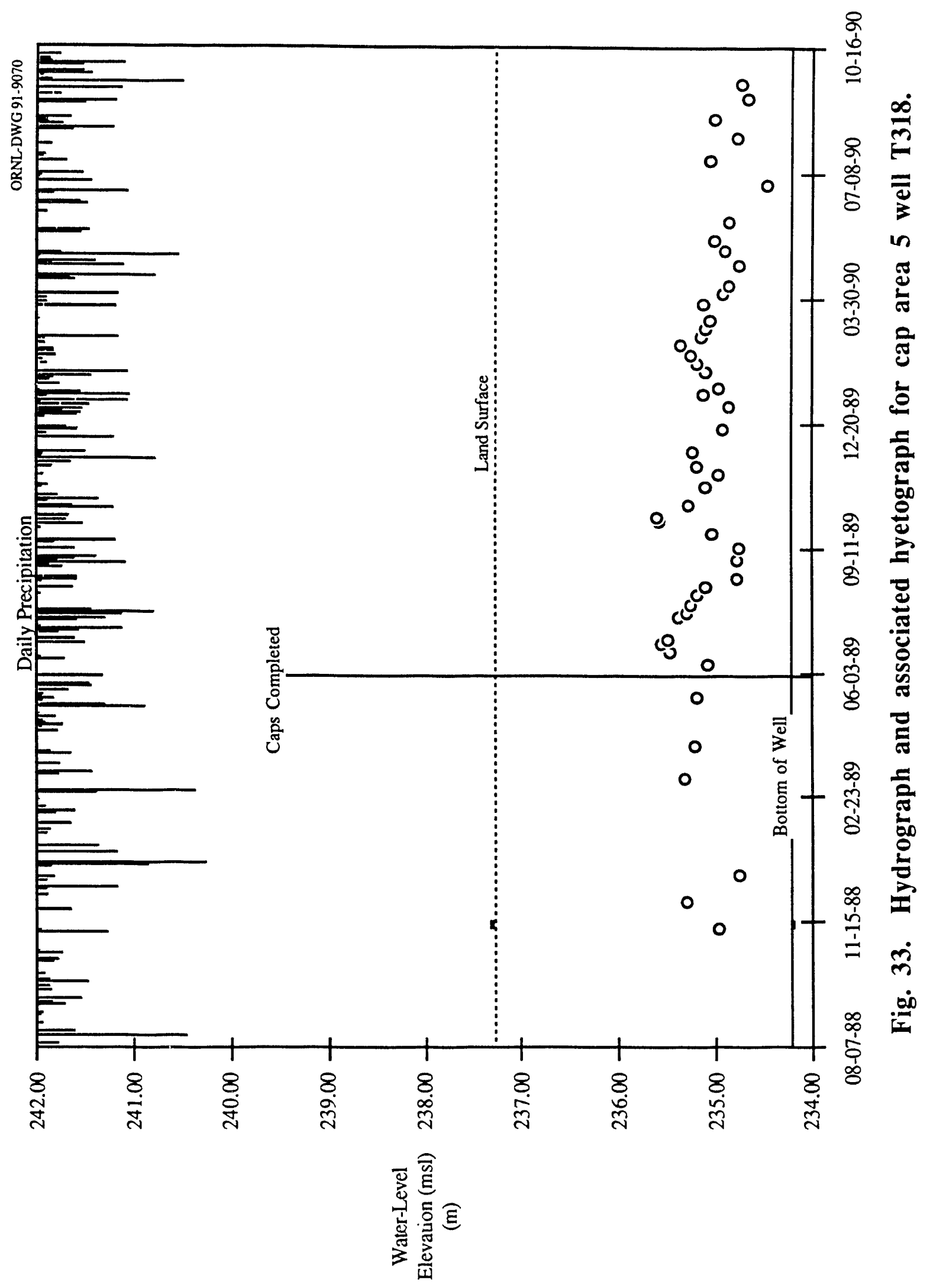




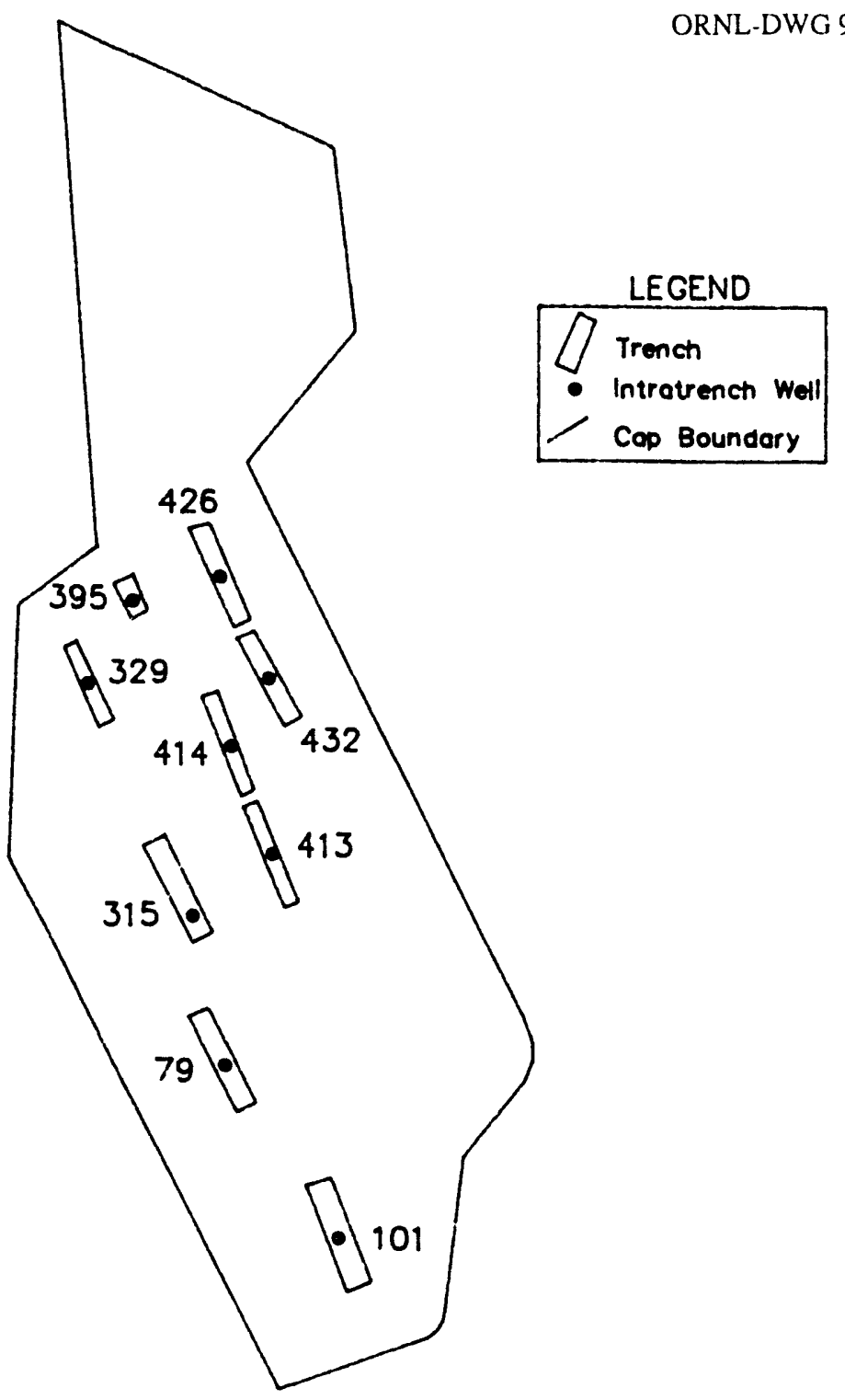

Fig. 34. Burial trench an ' well locations within cap area 6. 


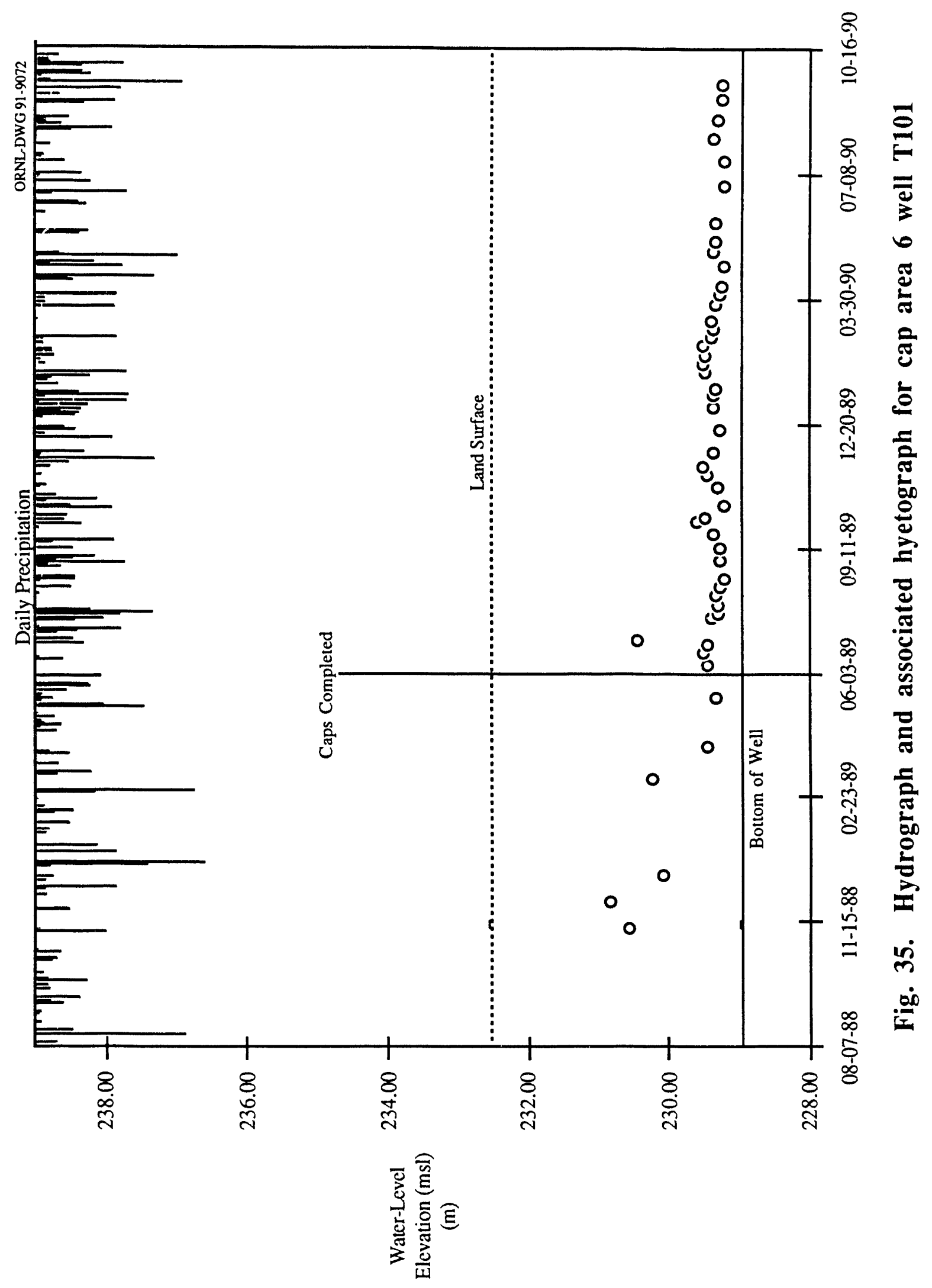




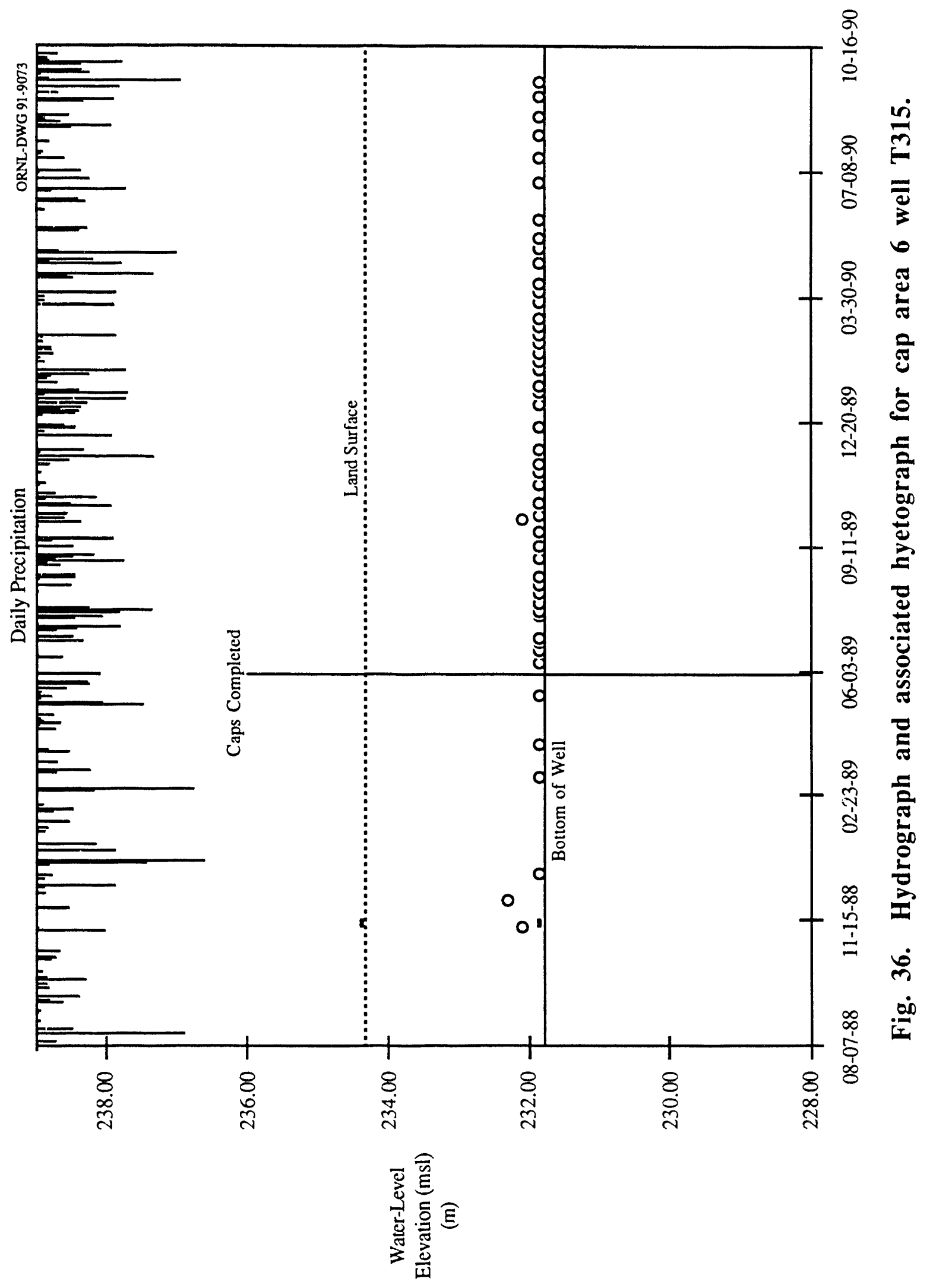




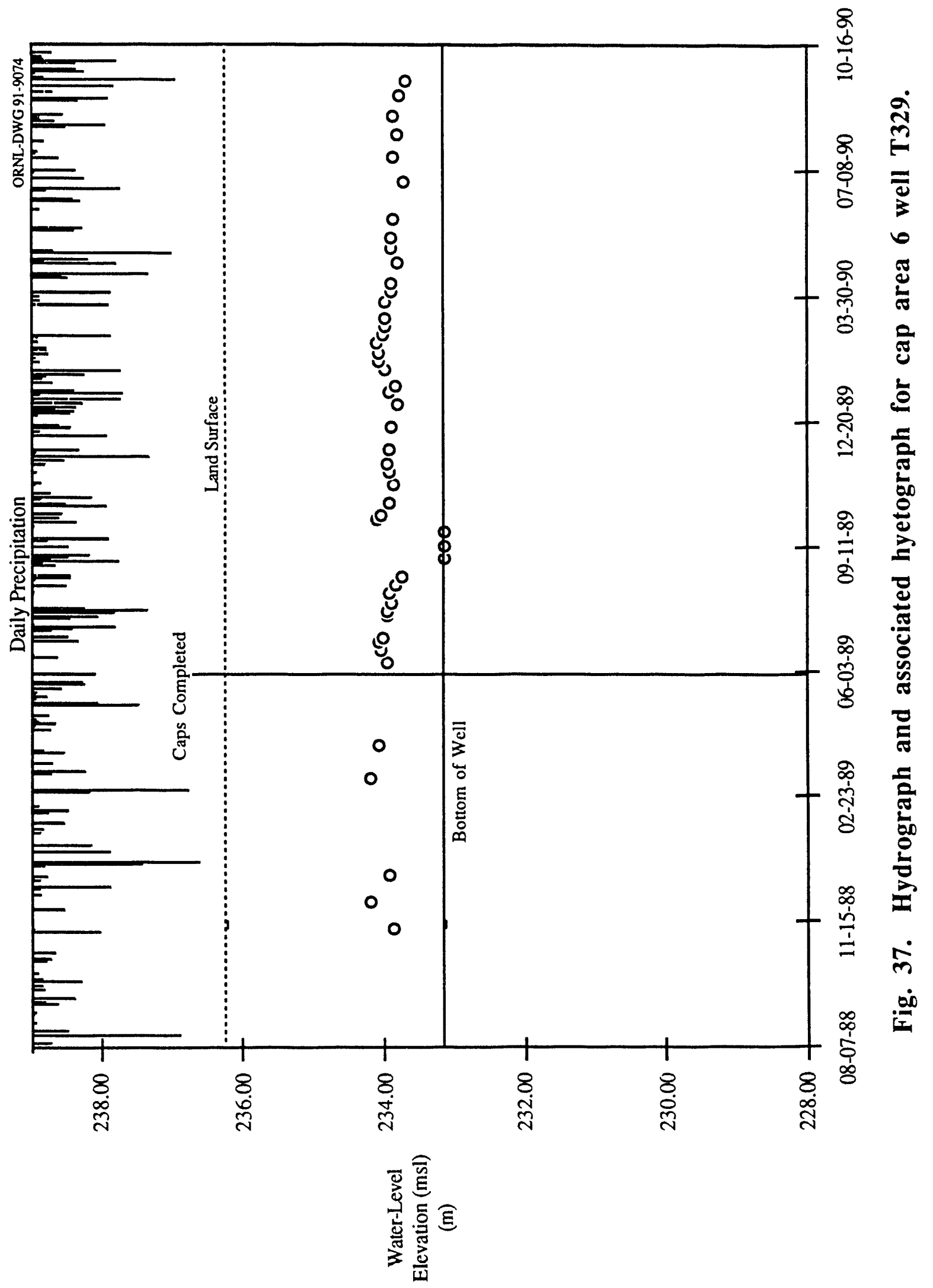




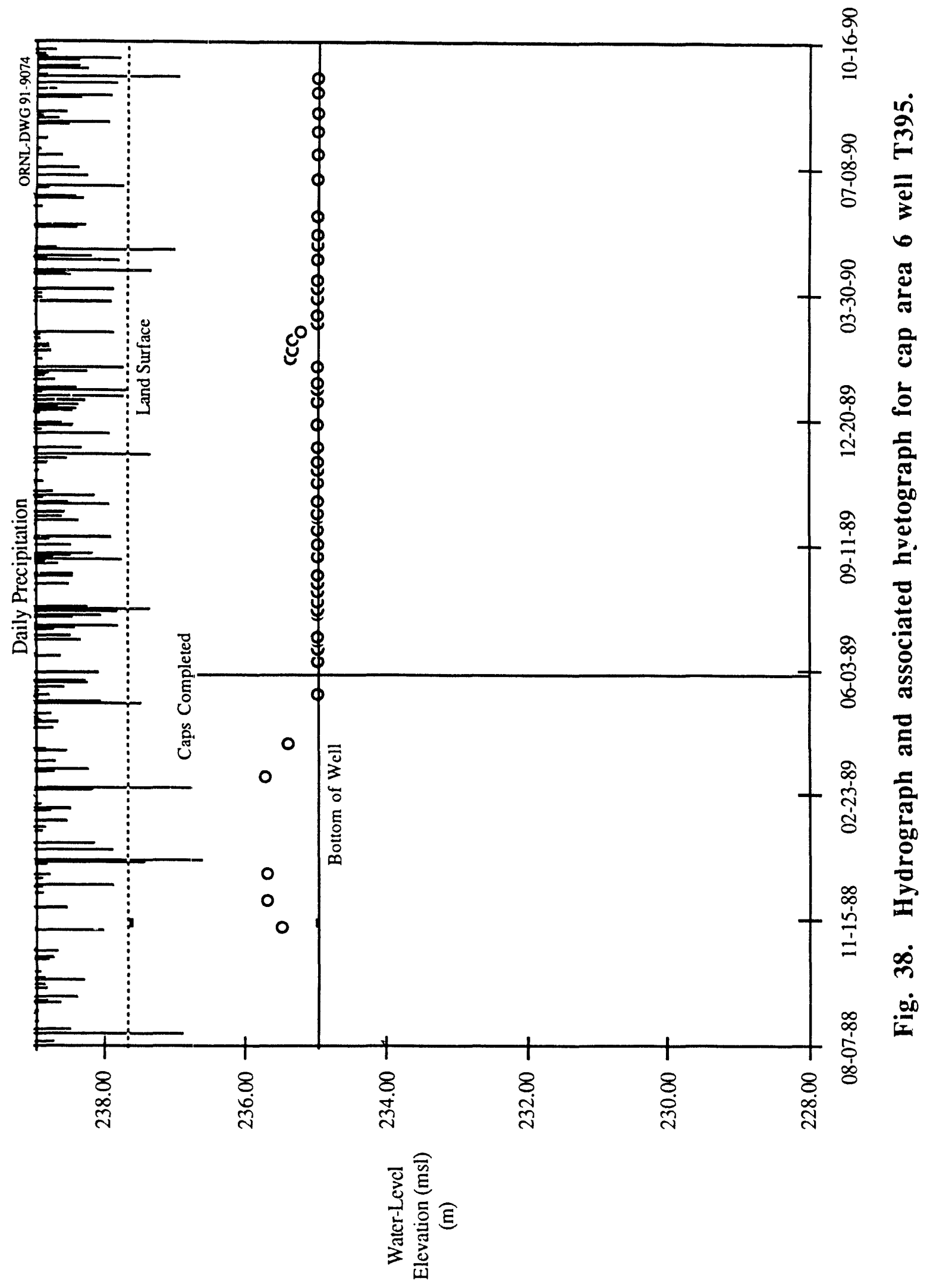




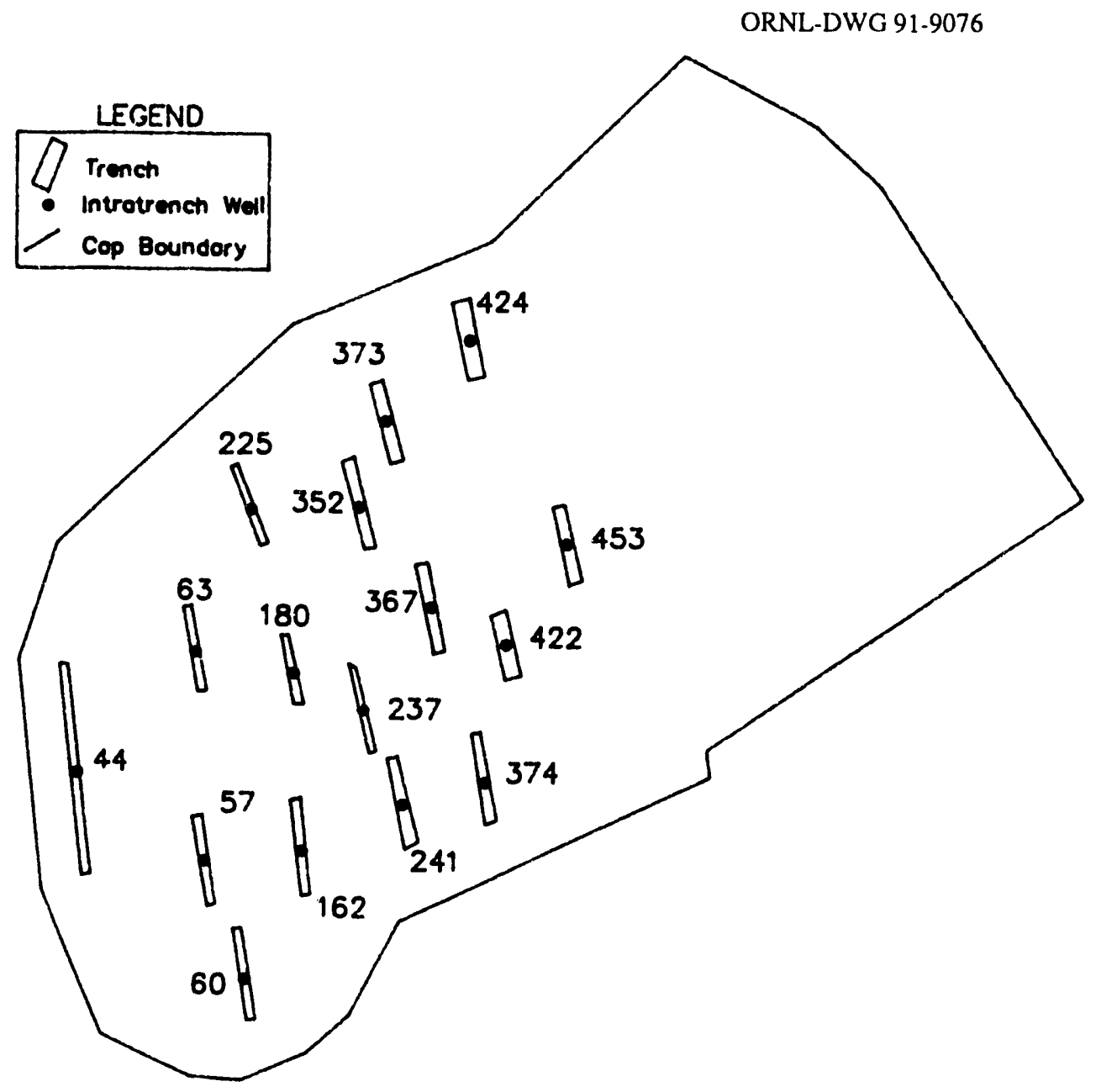

Fig. 39. Burial trench and well locations within cap area 8 .

57 


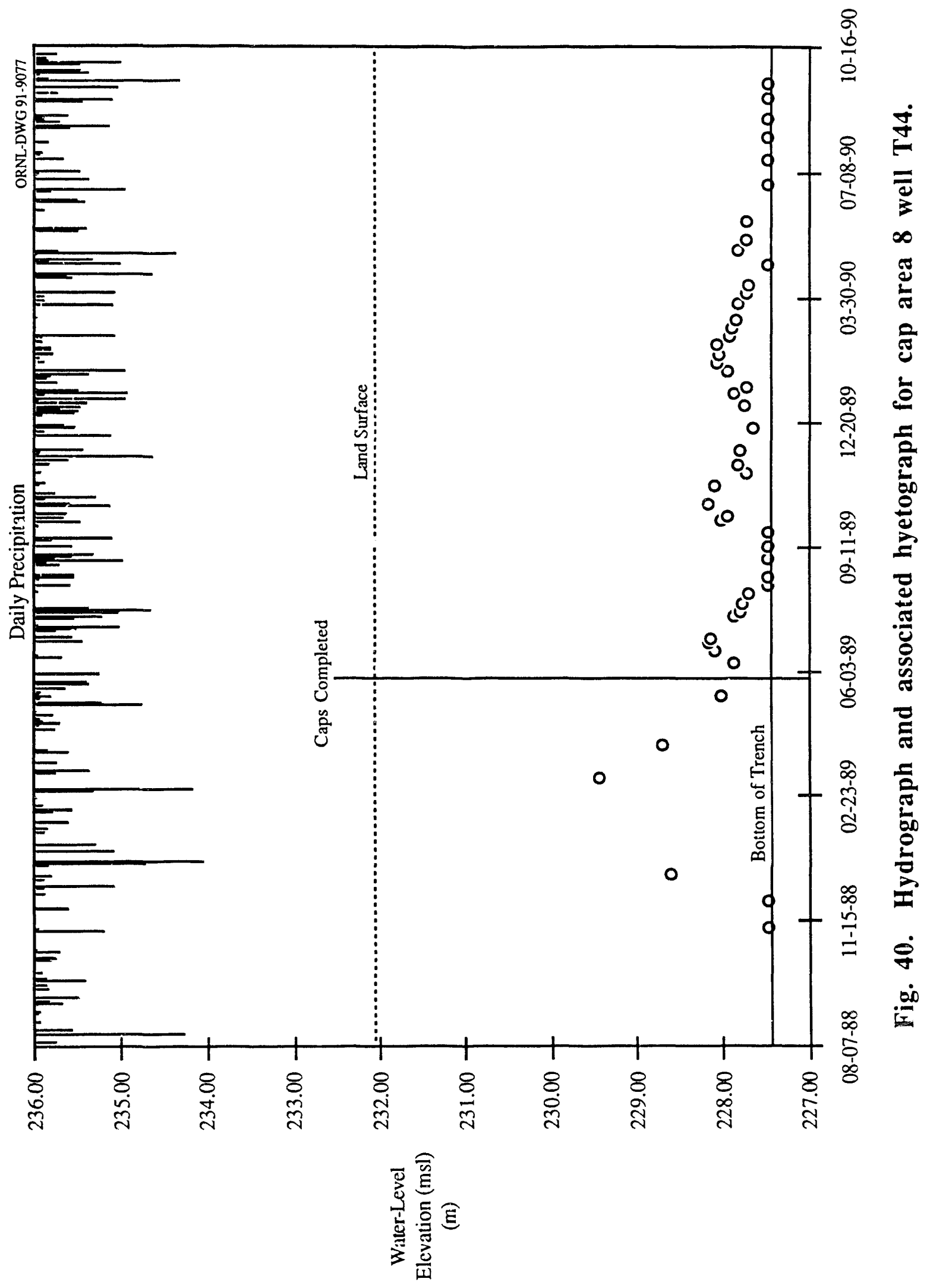




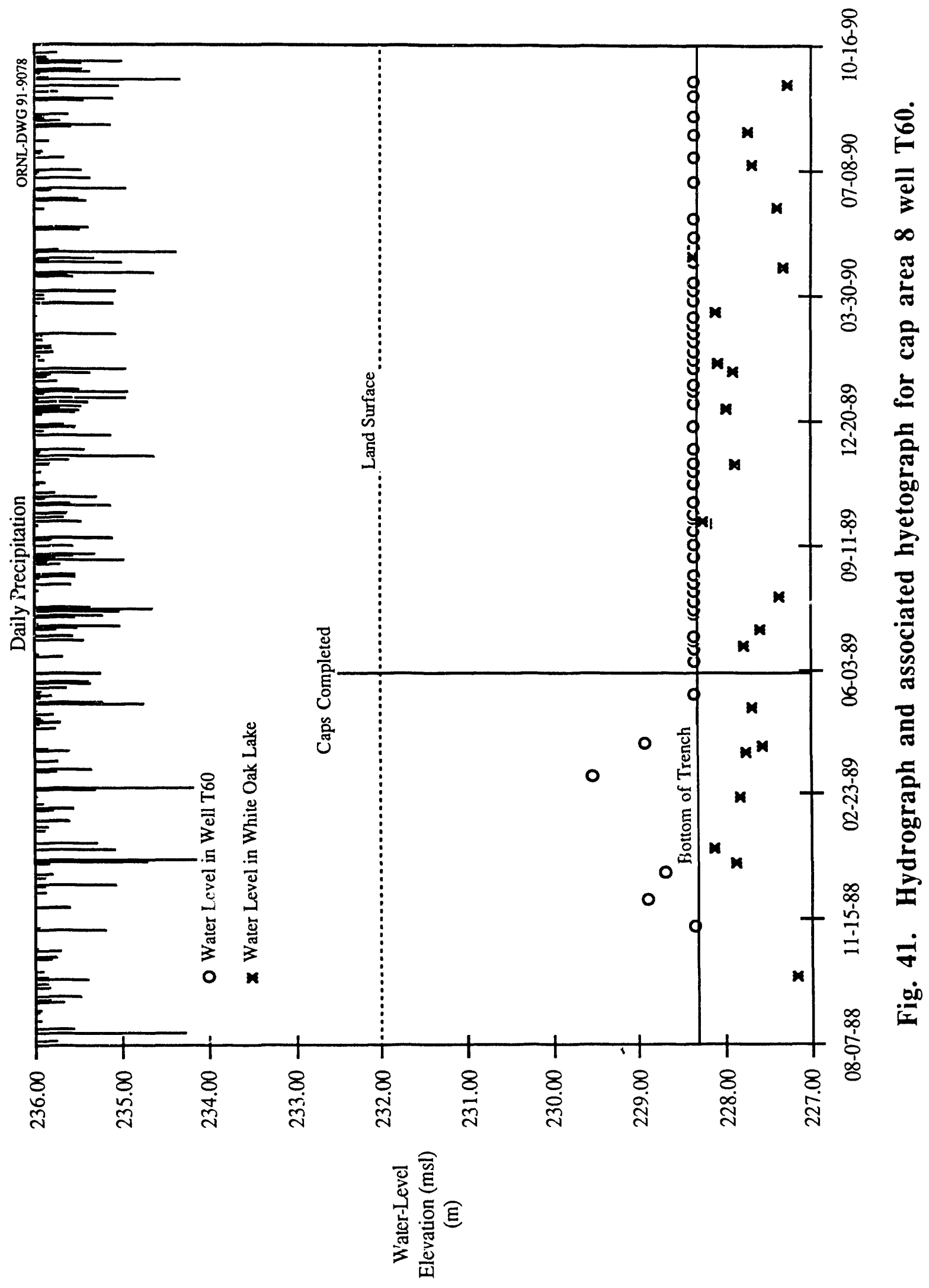




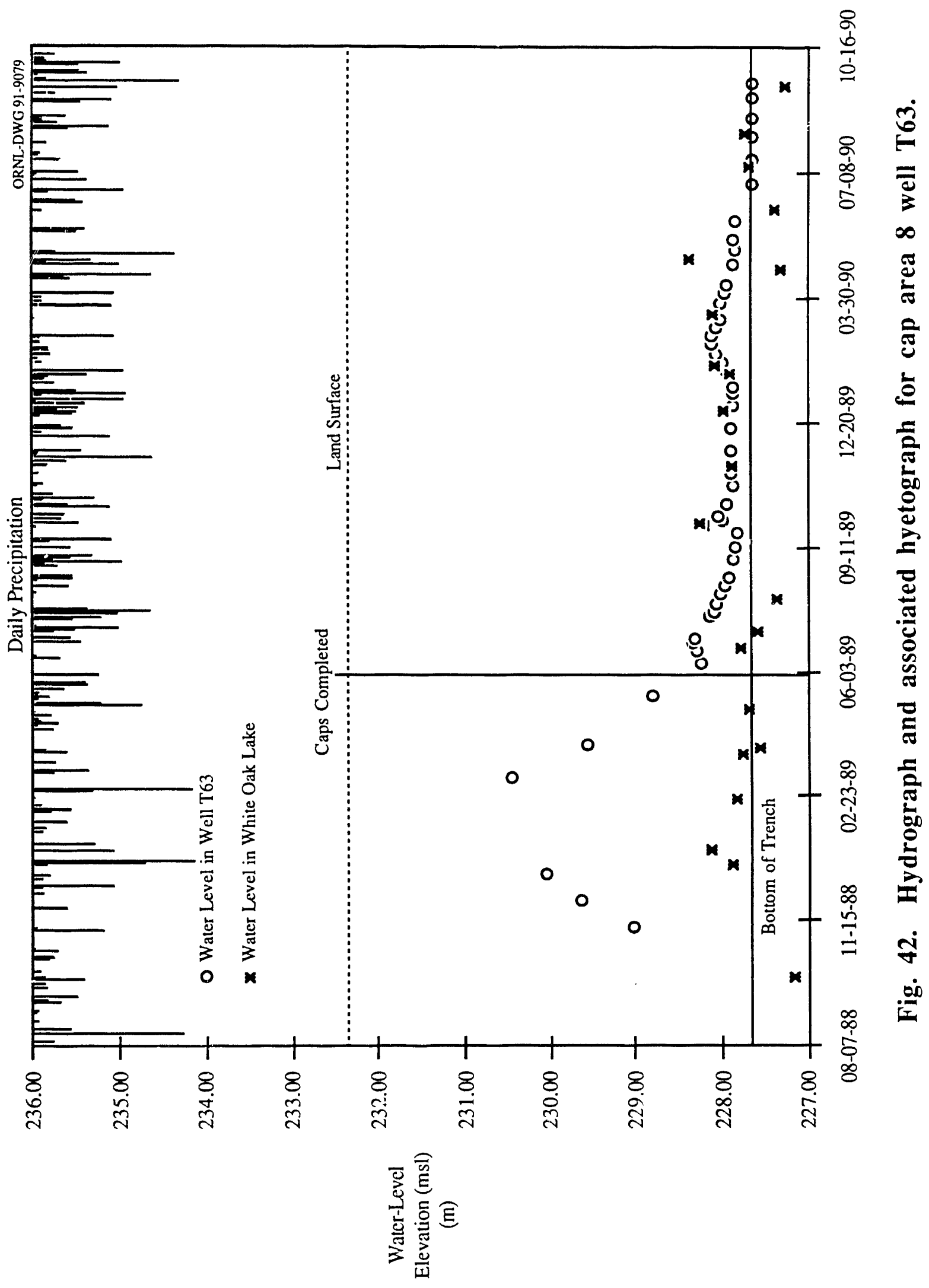




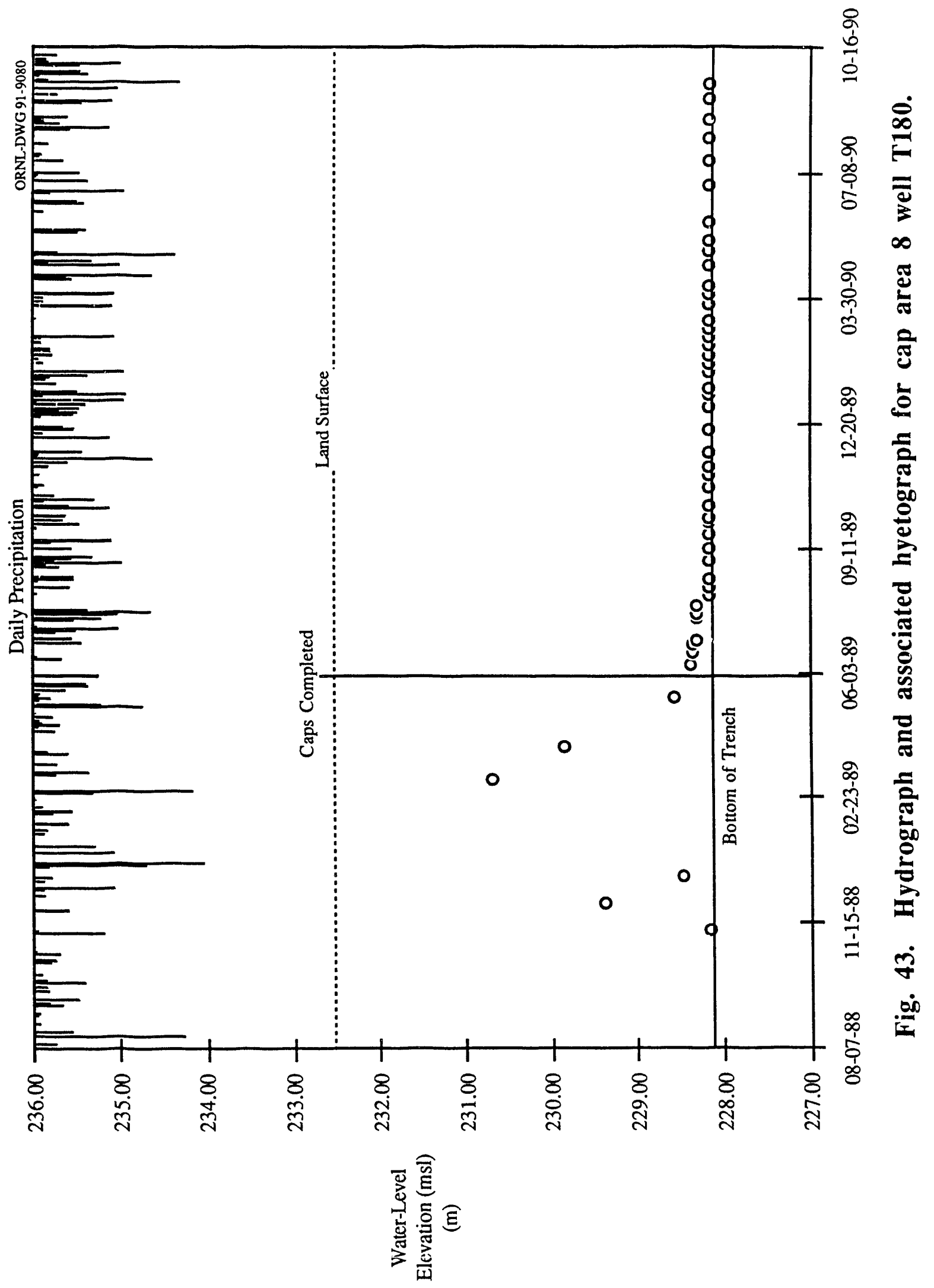




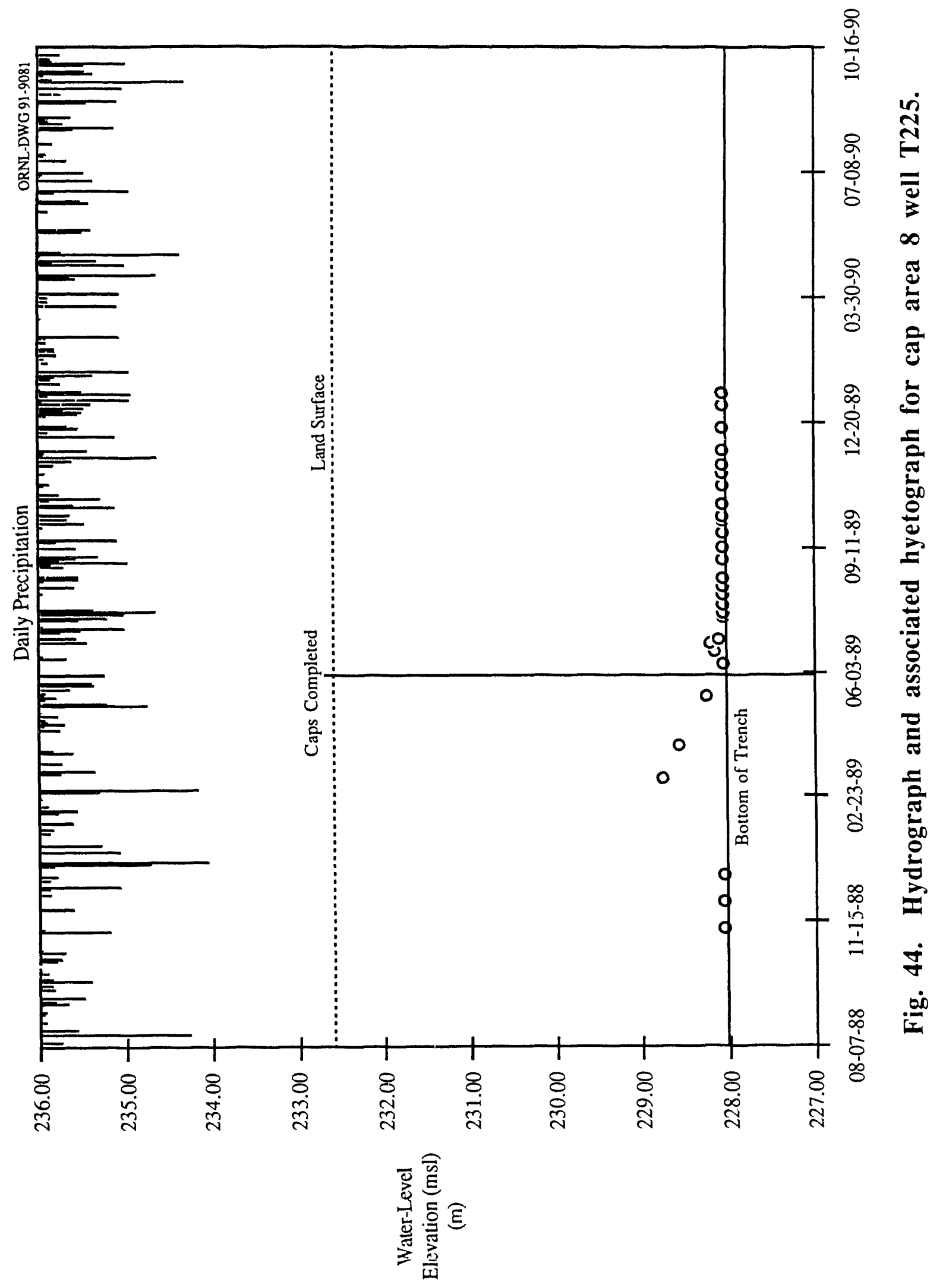




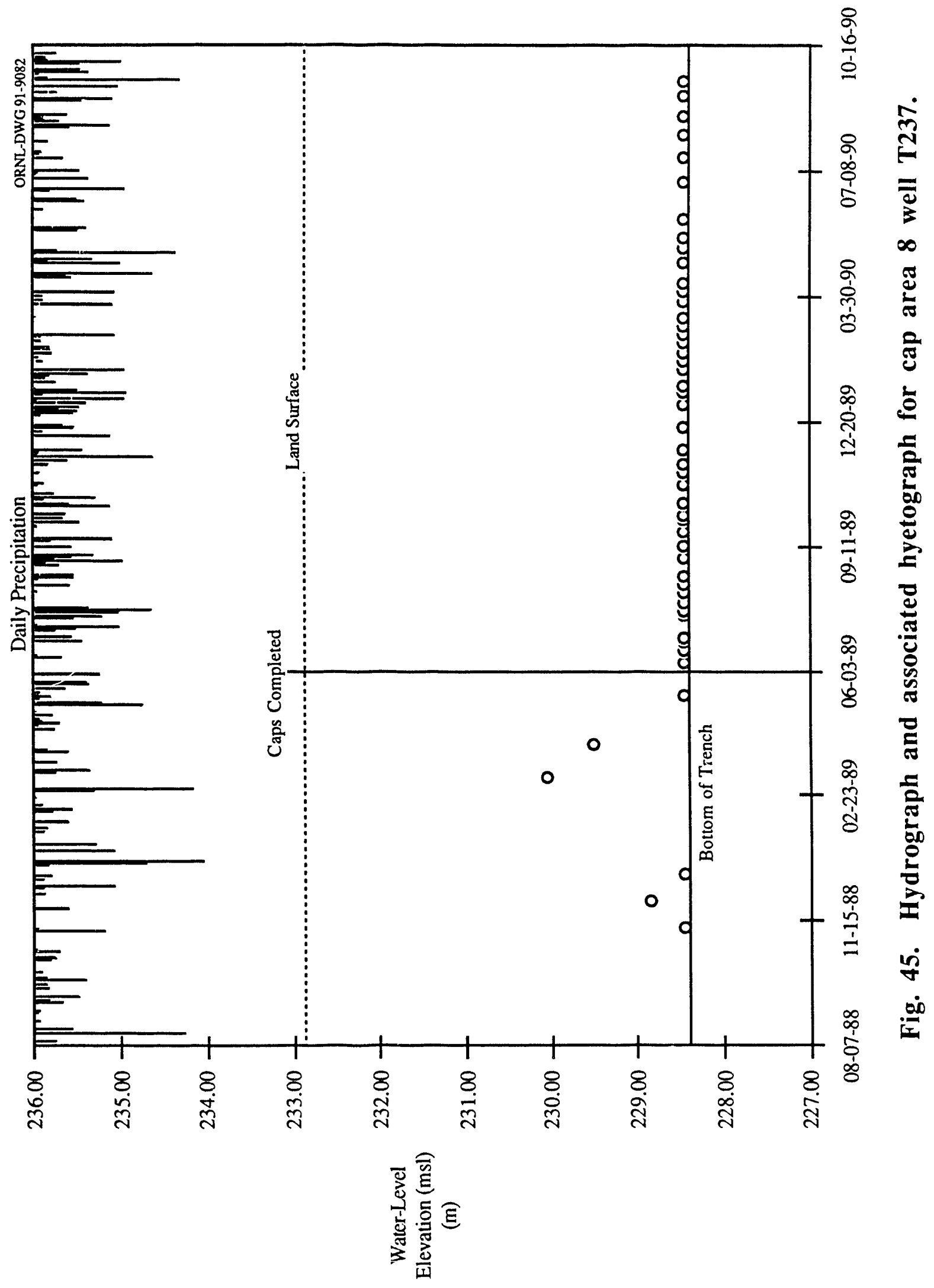




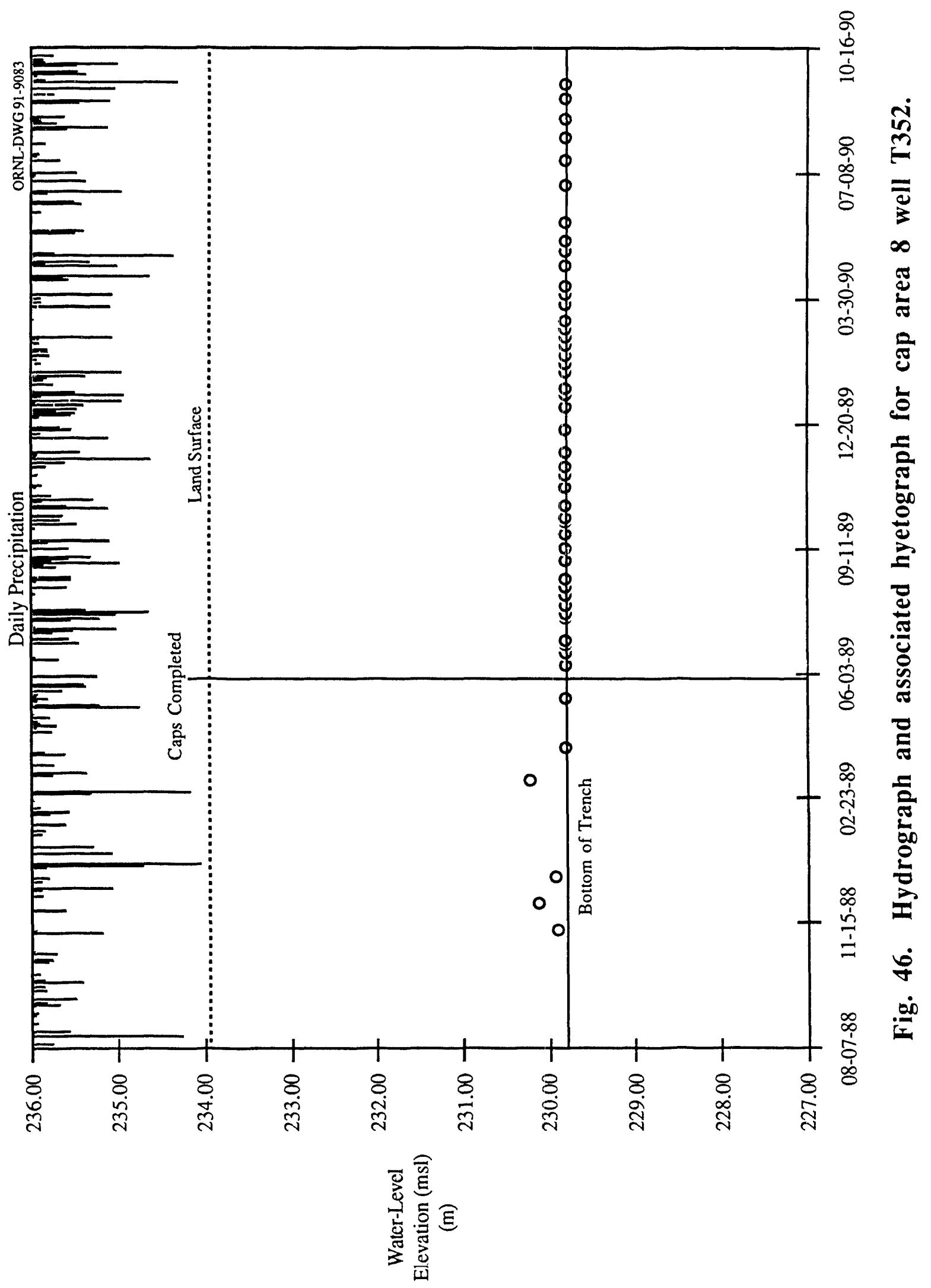




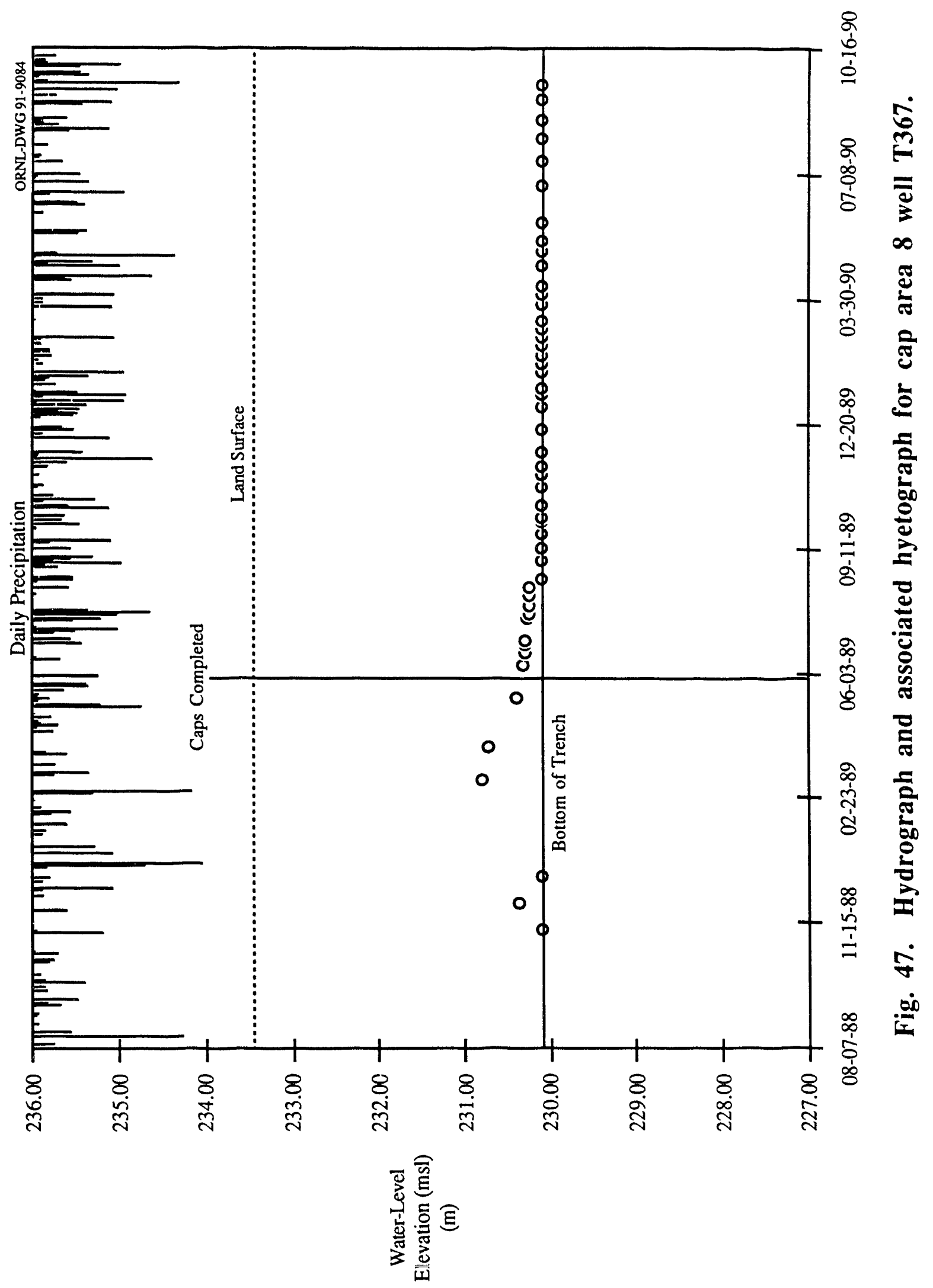




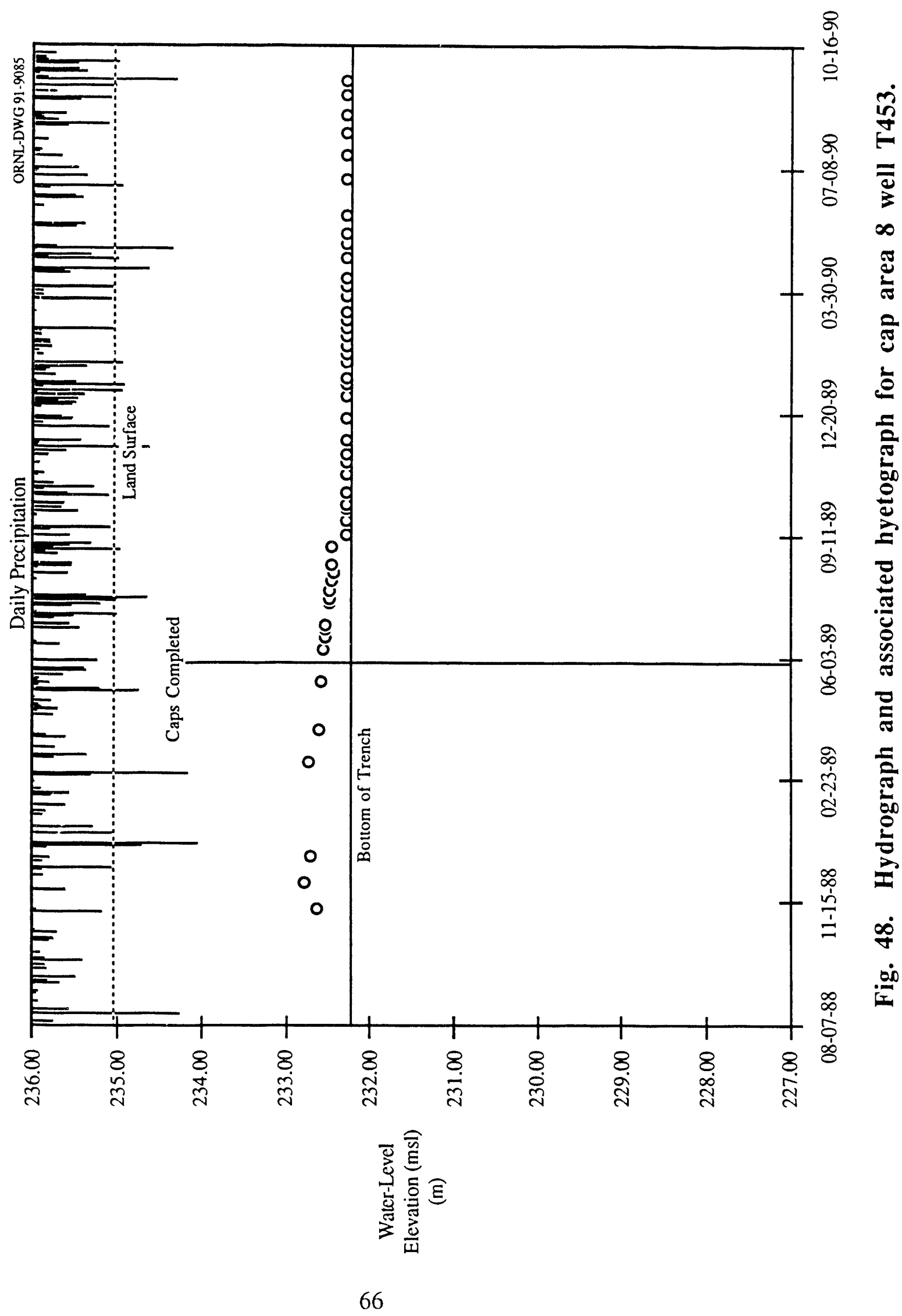


ORNL-DWG 91-9086

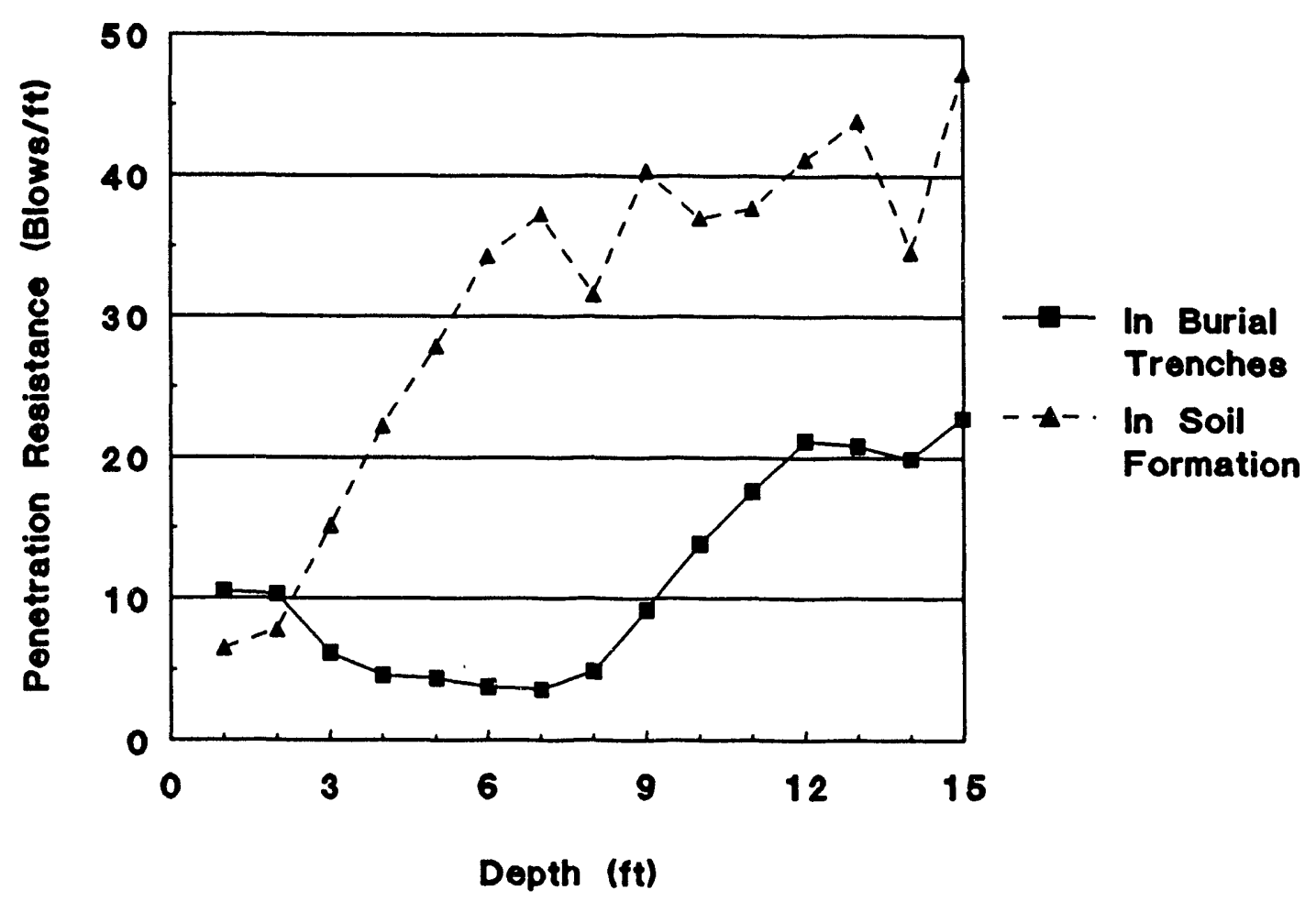

Fig. 49. Average penetration resistance of SWSA 6 burial trenches compared with the surrounding soil formation. 


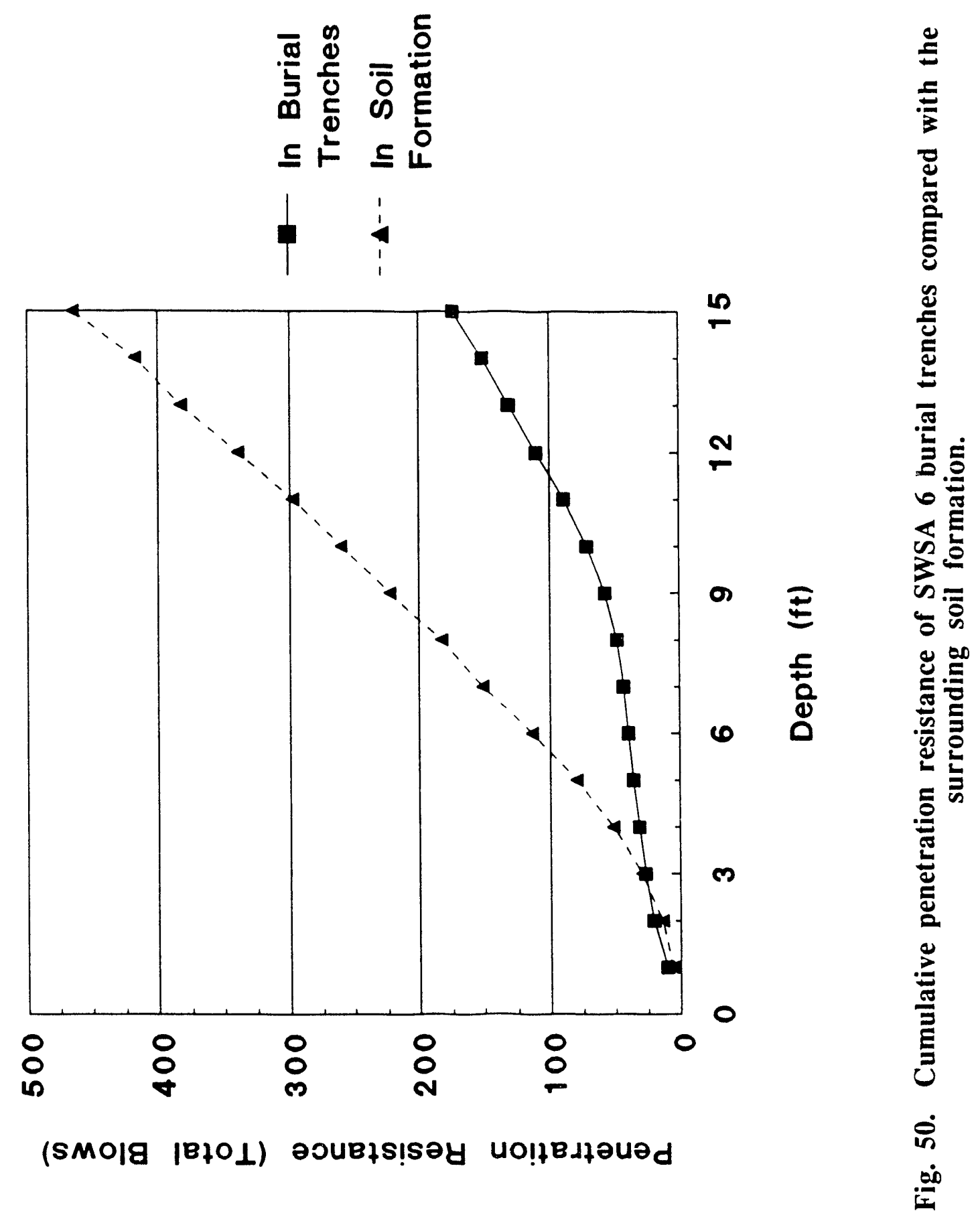




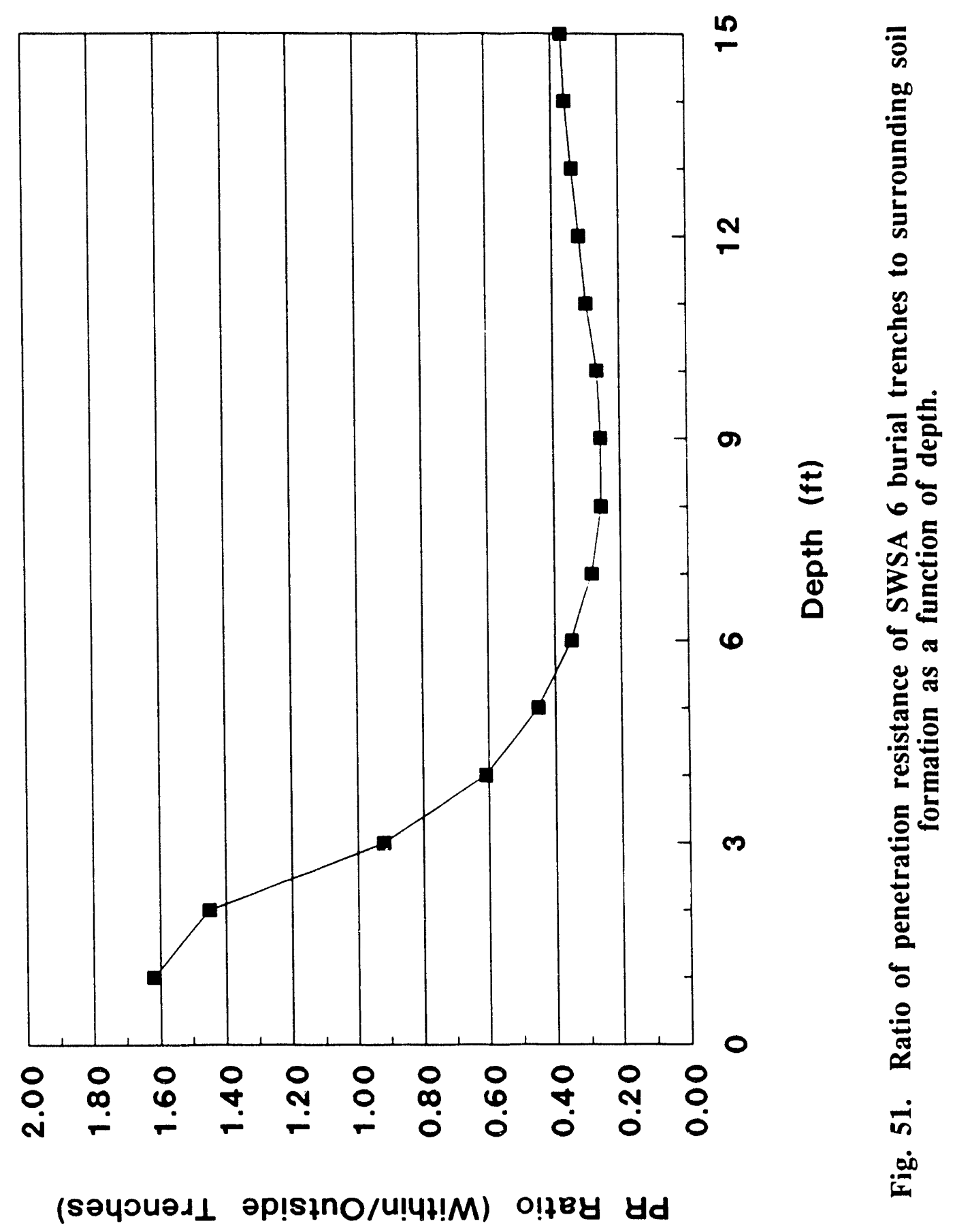


APPENDIX A: Water-level Data From Wells in and Around ICM Caps, Rainfall Data from SWSA 6, and Peak Monthly Elevations of White Oak Lake 
Table A.1. Depth to water and water-level elevations for ICM monitoring wells outside of burial trenches in SWSA 6

\begin{tabular}{|c|c|c|c|}
\hline $\begin{array}{l}\text { Well } \\
\text { No. }\end{array}$ & $\begin{array}{c}\text { Date } \\
\text { measured }\end{array}$ & $\begin{array}{l}\text { Depth to } \\
\text { water }(m)\end{array}$ & $\begin{array}{l}\text { Water-level } \\
\text { clevation }(\mathrm{m})\end{array}$ \\
\hline 276 & $10-04-88$ & 0.99 & 230.51 \\
\hline 276 & $10-24-88$ & 1.15 & 230.35 \\
\hline 276 & $11-01-88$ & 1.06 & 230.45 \\
\hline 276 & $11-07-88$ & 0.52 & 230.98 \\
\hline 276 & $11-14-88$ & 0.57 & 230.94 \\
\hline 276 & $11-21-88$ & 0.25 & 231.25 \\
\hline 276 & $11-28-88$ & 0.22 & 231.29 \\
\hline 276 & $12-06-88$ & 0.61 & 230.89 \\
\hline 276 & $12-12-88$ & 0.52 & 230.98 \\
\hline 276 & $12-20-88$ & 0.34 & 231.16 \\
\hline 276 & $12-27-88$ & 0.34 & 231.16 \\
\hline 276 & $01-02-89$ & 0.20 & 231.30 \\
\hline 276 & $01-11-89$ & 0.19 & 231.31 \\
\hline 276 & $01-17-89$ & 0.27 & 231.23 \\
\hline 276 & $01-27-89$ & 0.55 & 230.96 \\
\hline 276 & $01-31-89$ & 0.34 & 231.16 \\
\hline 276 & $02-10-89$ & 0.36 & 231.15 \\
\hline 276 & $02-17-89$ & 0.08 & 231.43 \\
\hline 276 & $02-24-89$ & 0.34 & 231.16 \\
\hline 276 & $03.03-89$ & 0.33 & 231.17 \\
\hline 276 & $03-15-89$ & 0.53 & 230.97 \\
\hline 276 & $03-22-89$ & 0.23 & 231.27 \\
\hline 276 & $03-28-89$ & 0.41 & 231.09 \\
\hline 276 & $04-07-89$ & 0.28 & 231.22 \\
\hline 276 & $04-14-89$ & 0.33 & 231.17 \\
\hline 276 & $04-22.89$ & 0.66 & 230.84 \\
\hline 276 & $05-04-89$ & 0.74 & 230.77 \\
\hline 276 & $05-12-89$ & 0.44 & 231.06 \\
\hline 276 & $05-17-89$ & 0.56 & 230.95 \\
\hline 276 & $05-26-89$ & 0.61 & 230.89 \\
\hline 276 & $06-01-89$ & 0.66 & 230.84 \\
\hline 276 & $06-09-89$ & 0.15 & 231.35 \\
\hline 276 & $06-16-89$ & 0.08 & 231.43 \\
\hline 276 & $06-23-89$ & 0.23 & 231.27 \\
\hline 276 & $06-28-89$ & 0.56 & 230.95 \\
\hline 276 & $07-07-89$ & 0.16 & 231.34 \\
\hline 276 & $07-14-89$ & 0.09 & 230.82 \\
\hline 276 & $07-21-89$ & 0.38 & 231.12 \\
\hline 276 & $07-28-89$ & 0.80 & 230.70 \\
\hline 276 & $08-04-89$ & 0.80 & 230.71 \\
\hline 276 & $08-11-89$ & 0.63 & 230.87 \\
\hline 276 & $08-18-89$ & 0.55 & 230.96 \\
\hline 276 & $08-30-89$ & 0.38 & 231.12 \\
\hline 276 & $09-07-89$ & 0.36 & 231.15 \\
\hline 276 & $09-18-89$ & 0.30 & 231.20 \\
\hline 276 & $09-27-89$ & 0.40 & 231.11 \\
\hline 276 & $01-15-90$ & 0.45 & 231.05 \\
\hline 276 & $02-15-90$ & 0.42 & 231.08 \\
\hline 276 & $03-14-90$ & 0.43 & 231.08 \\
\hline 276 & $04-23-90$ & 0.61 & 230.89 \\
\hline 276 & $05-21-90$ & 0.61 & 230.89 \\
\hline 276 & $06-26-90$ & 0.90 & 230.60 \\
\hline 276 & $07-25-90$ & 0.61 & 230.89 \\
\hline 276 & $08-23-90$ & 0.74 & 230.77 \\
\hline 276 & $09-25-90$ & 0.96 & 230.54 \\
\hline 318 & $10-04-88$ & Dry & 239.16 \\
\hline 318 & $10-24-88$ & Ury & 239.16 \\
\hline
\end{tabular}


Table A.1 (continued)

\begin{tabular}{|c|c|c|c|}
\hline $\begin{array}{l}\text { Well } \\
\text { No. }\end{array}$ & $\begin{array}{c}\text { Date } \\
\text { measured }\end{array}$ & $\begin{array}{l}\text { Depth to } \\
\text { water }(m)\end{array}$ & $\begin{array}{l}\text { Water-level } \\
\text { elevation }(m)\end{array}$ \\
\hline 318 & $11-01-88$ & Dry & 239.16 \\
\hline 318 & $11-07-88$ & 3.43 & 240.49 \\
\hline 318 & $11-14-88$ & Dry & 239.16 \\
\hline 318 & $11-21-88$ & 3.05 & 240.87 \\
\hline 318 & $11-28-88$ & 3.33 & 240.59 \\
\hline 318 & $12-06-88$ & Dry & 239.16 \\
\hline 318 & $12-12-88$ & Dry & 239.16 \\
\hline 318 & $12-20-88$ & Dry & 239.16 \\
\hline 318 & $12-27-88$ & 3.44 & 240.48 \\
\hline 318 & $01-02-89$ & 2.95 & 240.97 \\
\hline 318 & $01-11-89$ & 3.38 & 240.54 \\
\hline 318 & $01-17-89$ & 3.23 & 240.70 \\
\hline 318 & $01-27-89$ & Dry & 239.16 \\
\hline 318 & $01-31-89$ & Dry & 239.16 \\
\hline 318 & $02-10-89$ & 3.39 & 240.53 \\
\hline 318 & $02-17-89$ & 3.15 & 240.77 \\
\hline 318 & $02-24-89$ & Dry & 239.16 \\
\hline 318 & $03-03-89$ & 3.49 & 240.43 \\
\hline 318 & $03-15-89$ & 3.61 & 240.31 \\
\hline 318 & $03-22-89$ & 3.14 & 240.78 \\
\hline 318 & $03-28-89$ & 3.48 & 240.44 \\
\hline 318 & $04-07-89$ & 3.44 & 240.48 \\
\hline 318 & $04-14-89$ & 3.57 & 240.35 \\
\hline 318 & $04-22-89$ & Dry & 239.16 \\
\hline 318 & $05-04-89$ & Dry & 239.16 \\
\hline 318 & $05-12-89$ & 3.45 & 240.47 \\
\hline 318 & $05-17-89$ & 3.57 & 240.35 \\
\hline 318 & $05-28-89$ & 3.63 & 240.29 \\
\hline 318 & $06-01-89$ & 3.61 & 240.31 \\
\hline 318 & $06-09-89$ & 2.94 & 240.98 \\
\hline 318 & $06-16-89$ & 2.90 & 241.02 \\
\hline 318 & $06-23-89$ & 2.88 & 241.04 \\
\hline 318 & $06-2.8-89$ & 3.34 & 240.58 \\
\hline 318 & $07-07-89$ & 2.84 & 241.08 \\
\hline 318 & $07-14-89$ & 3.47 & 240.45 \\
\hline 318 & $07-21-89$ & 3.41 & 240.52 \\
\hline 318 & $07-28-89$ & 3.47 & 240.45 \\
\hline 318 & $08-04-89$ & 3.14 & 240.48 \\
\hline 318 & $08-11-89$ & 3.38 & 240.54 \\
\hline 318 & $08-18-89$ & 3.35 & 240.57 \\
\hline 318 & $08-30-89$ & 3.27 & 240.66 \\
\hline 318 & $09-07-89$ & 3.19 & 240.73 \\
\hline 318 & $09-18-89$ & 3.15 & 240 \\
\hline 318 & $09-27-89$ & 3.21 & 240.7 \\
\hline 318 & $01-15-90$ & 3.10 & 240.82 \\
\hline 318 & $02-15-90$ & Dry & 239.16 \\
\hline 318 & $03-14-90$ & Dry & 239.16 \\
\hline 318 & $04-23-90$ & Dry & 239.16 \\
\hline 318 & $05-21-90$ & Dry & 239.16 \\
\hline 318 & $06-26-90$ & Dry & 239.16 \\
\hline 318 & $07-25-90$ & Dry & 239.16 \\
\hline 318 & $08-23-90$ & Dry & 239.16 \\
\hline 318 & $09-25-90$ & Dry & 239.16 \\
\hline 345 & $01-02-89$ & 1.01 & 229.86 \\
\hline 345 & $01-11-89$ & 1.12 & 229.75 \\
\hline 345 & $01-17-89$ & 1.00 & 229.87 \\
\hline 345 & $01-27-89$ & 1.15 & 229.72 \\
\hline 345 & $01-31-89$ & 1.12 & 229.75 \\
\hline
\end{tabular}


Table A.1 (continued)

\begin{tabular}{|c|c|c|c|}
\hline $\begin{array}{l}\text { Well } \\
\text { No. }\end{array}$ & $\begin{array}{c}\text { Date } \\
\text { measured }\end{array}$ & $\begin{array}{l}\text { Depth to } \\
\text { water }(\mathrm{m})\end{array}$ & $\begin{array}{l}\text { Water-level } \\
\text { elevation }(\mathrm{m})\end{array}$ \\
\hline 345 & $02-10-89$ & 1.11 & 229.77 \\
\hline 345 & $02-17-89$ & 0.99 & 229.88 \\
\hline 345 & $02-24-89$ & 1.07 & 229.80 \\
\hline 345 & $03-03-89$ & 1.20 & 229.68 \\
\hline 345 & $03-15-89$ & 1.27 & 229.60 \\
\hline 3.15 & $03-22-89$ & 1.17 & 229.70 \\
\hline 345 & $03-28-89$ & 1.23 & 229.64 \\
\hline 345 & 04-07-89 & 1.22 & 229.65 \\
\hline 345 & $04-14-89$ & 1.24 & 229.63 \\
\hline 345 & $04-22-89$ & 1.34 & 229.54 \\
\hline 345 & $05-04-89$ & 1.31 & 229.56 \\
\hline 345 & $05-12-89$ & 1.17 & 229.70 \\
\hline 345 & $05-17-89$ & 1.24 & 229.63 \\
\hline 345 & $05-26-89$ & 1.27 & 229.60 \\
\hline 345 & $06-01-89$ & 1.28 & 229.59 \\
\hline 345 & $06-09-89$ & 0.89 & 229.98 \\
\hline 345 & $06-16-89$ & 0.90 & 229.97 \\
\hline 345 & $06-23-89$ & 0.91 & 229.96 \\
\hline 345 & $06-28-89$ & 1.16 & 229.72 \\
\hline 345 & 07-07-89 & 0.95 & 229.92 \\
\hline 345 & $07-14-89$ & 1.17 & 229.70 \\
\hline 345 & $07-21-89$ & 1.17 & 229.70 \\
\hline 345 & $07-28-89$ & 1.36 & 229.51 \\
\hline 345 & $08-04-89$ & 1.27 & 229.60 \\
\hline 345 & $08-11-89$ & 1.22 & 229.65 \\
\hline 345 & $08-18-89$ & 1.20 & 229.67 \\
\hline 345 & $08-30-89$ & 1.02 & 229.86 \\
\hline 345 & $09-07-89$ & 1.02 & 229.85 \\
\hline 345 & $09-18-89$ & 1.02 & 229.86 \\
\hline 345 & $09-27-89$ & 1.02 & 229.85 \\
\hline 345 & $01-15-90$ & 0.89 & 229.98 \\
\hline 345 & $02-15-90$ & 1.09 & 229.78 \\
\hline 345 & $03-14-90$ & 1.13 & 229.74 \\
\hline 345 & $04-23-90$ & 1.25 & 229.62 \\
\hline 345 & $05-21-90$ & 1.09 & 229.78 \\
\hline 345 & $06-26-90$ & 1.17 & 229.70 \\
\hline 345 & $07-25-90$ & 1.16 & 229.71 \\
\hline 345 & $08-23-90$ & 1.11 & 229.76 \\
\hline 345 & $09-25-90$ & $1.2 \vdots$ & 229.62 \\
\hline 347 & $12-27-88$ & 0.82 & 236.24 \\
\hline 347 & $01-02-89$ & 0.23 & 236.83 \\
\hline 347 & $01-11-89$ & 0.30 & 236.76 \\
\hline 347 & $01-17-89$ & 0.19 & 236.87 \\
\hline 347 & $01-27-89$ & 0.75 & 236.31 \\
\hline 347 & $01-31-89$ & 0.74 & 236.32 \\
\hline 347 & $02-10-89$ & 0.61 & 236.45 \\
\hline 347 & $02-17-89$ & 0.08 & 236.98 \\
\hline 347 & $02-24-89$ & 0.33 & 236.73 \\
\hline 347 & $04-22-89$ & 2.27 & 234.79 \\
\hline 347 & $05-04-89$ & 2.13 & 235.16 \\
\hline 347 & $05-12-89$ & 3.20 & 234.10 \\
\hline 347 & $05-17-89$ & 1.97 & 235.33 \\
\hline 347 & $05-20-89$ & 1.98 & 235.32 \\
\hline 347 & $06-01-89$ & 2.01 & 235.29 \\
\hline 347 & $06-09-89$ & 1.73 & 23.5 .57 \\
\hline $3 .+7$ & $06-16-89$ & 1.71 & 235.59 \\
\hline 347 & $06-23-89$ & 1.35 & 235.95 \\
\hline
\end{tabular}


Table A.1 (continued)

\begin{tabular}{|c|c|c|c|}
\hline $\begin{array}{l}\text { Well } \\
\text { No. }\end{array}$ & $\begin{array}{c}\text { Date } \\
\text { measured }\end{array}$ & $\begin{array}{l}\text { Derth to } \\
\text { water }(m)\end{array}$ & $\begin{array}{l}\text { Water-level } \\
\text { elevation }(m)\end{array}$ \\
\hline 347 & $06-28-89$ & 1.46 & 235.84 \\
\hline 347 & $07-07-89$ & 1.41 & 235.89 \\
\hline 347 & $07-14-89$ & 1.55 & 235.75 \\
\hline 347 & $07-21-89$ & 1.56 & 235.73 \\
\hline 347 & $07-28-89$ & 2.01 & 235.29 \\
\hline 347 & $08-04-89$ & 1.92 & 235.38 \\
\hline 347 & $08-11-89$ & 1.91 & 235.39 \\
\hline 347 & $08-18-89$ & 1.91 & 235.39 \\
\hline 347 & $08-30-89$ & 1.58 & 235.72 \\
\hline 347 & $09-07-89$ & 1.56 & 235.73 \\
\hline 347 & $09-18-89$ & 1.59 & 235.71 \\
\hline 347 & $09-27-89$ & 1.59 & 235.71 \\
\hline 347 & $01-15-90$ & 2.19 & 235.11 \\
\hline 347 & $02-15-90$ & 1.80 & 235.50 \\
\hline 347 & $03-14-90$ & 2.06 & 235.23 \\
\hline 347 & $04-23-90$ & 2.33 & 234.97 \\
\hline 347 & $05-21-90$ & 2.03 & 235.27 \\
\hline 347 & $06 \cdot 26-90$ & 2.67 & 234.63 \\
\hline 347 & $07-25-90$ & 1.81 & 235.48 \\
\hline 347 & $08-23-90$ & 2.08 & 235.22 \\
\hline 347 & $09-25-90$ & 2.71 & 234.59 \\
\hline 356 & $10-04-88$ & 3.42 & 232.17 \\
\hline 356 & $10-24-88$ & 4.09 & 231.51 \\
\hline 356 & $11-01-88$ & 4.19 & 231.40 \\
\hline 356 & $11-07-88$ & 3.05 & 232.55 \\
\hline 356 & $11-14-88$ & 3.18 & 232.42 \\
\hline 356 & $11-21-88$ & 2.91 & 232.69 \\
\hline 356 & $11-28-88$ & 3.01 & 232.58 \\
\hline 356 & $12-06-88$ & 3.34 & 232.26 \\
\hline 356 & $12-12-88$ & 3.51 & 232.09 \\
\hline 356 & $12-20-88$ & 3.68 & 231.91 \\
\hline 356 & $12-27-88$ & 3.20 & 232.39 \\
\hline 356 & $01-02-89$ & 2.97 & 232.63 \\
\hline 356 & $01-11-89$ & 3.06 & 232.53 \\
\hline 356 & $01-17-89$ & 2.94 & 232.66 \\
\hline 356 & $01-27-89$ & 3.38 & 232.22 \\
\hline 356 & $01-31-89$ & 3.34 & 232.25 \\
\hline 356 & $02-10-89$ & 2.60 & 232.99 \\
\hline 356 & $02-17-89$ & 3.13 & 232.47 \\
\hline 356 & $02-24-89$ & 3.24 & 232.36 \\
\hline 356 & $03-03-89$ & 3.12 & 232.48 \\
\hline 356 & $03-15-89$ & 3.36 & 232.23 \\
\hline 356 & $03-22-89$ & 3.14 & 232.46 \\
\hline 356 & $03-28-89$ & 3.30 & 232.29 \\
\hline 356 & $04-07-89$ & 3.28 & 232.31 \\
\hline 356 & $04-14-89$ & 3.30 & 232.29 \\
\hline 356 & $04-22-89$ & 3.63 & 231.96 \\
\hline 356 & $05-04-89$ & 3.71 & 231.88 \\
\hline 356 & $05-12-89$ & 3.38 & 232.22 \\
\hline 356 & $05-17.89$ & 3.51 & 232.09 \\
\hline 356 & $05-26-89$ & 3.61 & 231.99 \\
\hline 356 & $06-01-89$ & 3.58 & 232.01 \\
\hline 356 & $06-09-89$ & 3.05 & 232.55 \\
\hline 356 & $06-16-89$ & 3.07 & 232.52 \\
\hline 356 & $06-23-89$ & 2.80 & 232.80 \\
\hline 356 & $06-28-89$ & 3.25 & 232.34 \\
\hline 356 & $07-07-89$ & 2.80 & 232.80 \\
\hline 356 & $07-14-89$ & 3.35 & 232.24 \\
\hline
\end{tabular}


Table A.1 (continued)

\begin{tabular}{|c|c|c|c|}
\hline $\begin{array}{l}\text { Well } \\
\text { No. }\end{array}$ & $\begin{array}{c}\text { Date } \\
\text { measured }\end{array}$ & $\begin{array}{l}\text { Depth to } \\
\text { water }(m)\end{array}$ & $\begin{array}{l}\text { Water-level } \\
\text { elevation }(\mathrm{m})\end{array}$ \\
\hline 356 & $07-21-89$ & 3.38 & 232.22 \\
\hline 356 & $07-28-89$ & 3.71 & 231.88 \\
\hline 356 & $08-04-89$ & 3.57 & 232.02 \\
\hline 356 & $08-11-89$ & 3.56 & 232.04 \\
\hline 356 & $08-18-89$ & 3.51 & 232.09 \\
\hline 356 & $08-30-89$ & 3.05 & 232.55 \\
\hline 356 & $09-07-89$ & 3.10 & 232.49 \\
\hline 356 & $09-18-89$ & 3.05 & 232.55 \\
\hline 356 & $09-27-89$ & 3.11 & 232.48 \\
\hline 356 & $01-15-90$ & 3.41 & 232.18 \\
\hline 356 & $02-15-90$ & 3.33 & 232.26 \\
\hline 356 & $03-14-90$ & 3.44 & 232.16 \\
\hline 356 & $04-23-90$ & 3.79 & 231.80 \\
\hline 356 & $05-21-90$ & 3.62 & 231.97 \\
\hline 356 & $06-26-90$ & 3.99 & 231.60 \\
\hline 356 & $07-25-90$ & 3.29 & 232.31 \\
\hline 356 & $08-23-90$ & 3.58 & 232.02 \\
\hline 356 & $09-25-90$ & 3.96 & 231.63 \\
\hline 368 & $01-02-89$ & 7.60 & 242.25 \\
\hline 368 & $01-11-89$ & 6.10 & 243.75 \\
\hline 368 & $01-17-89$ & 7.44 & 242.41 \\
\hline 368 & $01-27-89$ & 7.60 & 242.25 \\
\hline 368 & $01-31-89$ & 7.65 & 242.20 \\
\hline 368 & $03-15-89$ & 7.62 & 242.23 \\
\hline 368 & $03-22-89$ & 7.75 & 242.09 \\
\hline 368 & $03-28-89$ & 7.79 & 242.06 \\
\hline 368 & $04-07-89$ & 7.70 & 242.14 \\
\hline 368 & $04-22-89$ & 8.42 & 241.42 \\
\hline 368 & $05-04-89$ & 8.46 & 241.98 \\
\hline 368 & $05-12-89$ & 8.33 & 242.11 \\
\hline 368 & $05-17-89$ & 8.28 & 242.16 \\
\hline 368 & $05-26-89$ & 8.35 & 242.10 \\
\hline 368 & $06-01-89$ & 8.33 & 242.12 \\
\hline 368 & $06-09-89$ & 8.28 & 242.16 \\
\hline 368 & $06-16-89$ & 8.21 & 242.23 \\
\hline 368 & $06-23-89$ & 8.18 & 242.27 \\
\hline 368 & $06-28-89$ & 8.22 & 242.23 \\
\hline 368 & $07-07-89$ & 8.22 & 242.23 \\
\hline 368 & $07-14-89$ & 8.31 & 242.14 \\
\hline 368 & $07-21-89$ & 8.54 & 241.91 \\
\hline 368 & $07-28-89$ & 8.40 & 242.05 \\
\hline 368 & $08-04-89$ & 8.56 & 241.89 \\
\hline 368 & $08-11-89$ & 8.54 & 241.91 \\
\hline 368 & $08-18-89$ & 8.52 & 241.93 \\
\hline 368 & $08-30-89$ & 8.18 & 242.27 \\
\hline 368 & $09-07-89$ & 8.21 & 242.24 \\
\hline 368 & $09-18-89$ & 8.14 & 242.30 \\
\hline 368 & $09-27-89$ & 8.22 & 242.23 \\
\hline 368 & $01-15-90$ & 8.25 & 242.19 \\
\hline 368 & $02-15-90$ & 8.17 & 242.27 \\
\hline 368 & $03-14-90$ & 8.23 & 242.21 \\
\hline 368 & $04-23-90$ & 8.30 & 242.14 \\
\hline 368 & $05-21-90$ & 8.27 & 242.17 \\
\hline 368 & $06-26-90$ & 8.34 & 242.11 \\
\hline 368 & $07-25-90$ & 8.30 & 242.14 \\
\hline 368 & 0823.90 & 8.33 & 242.12 \\
\hline 368 & $09-25-90$ & 8.40 & 242.05 \\
\hline 636 & $01-12.88$ & 10.12 & 244.36 \\
\hline
\end{tabular}


Table A.1 (continued)

\begin{tabular}{|c|c|c|c|}
\hline $\begin{array}{l}\text { Well } \\
\text { No. }\end{array}$ & $\begin{array}{c}\text { Date } \\
\text { measured }\end{array}$ & $\begin{array}{l}\text { Depth to } \\
\text { water }(m)\end{array}$ & $\begin{array}{l}\text { Water-level } \\
\text { elevation }(m)\end{array}$ \\
\hline 636 & $02-04-88$ & 9.72 & 244.76 \\
\hline 636 & $03-03-88$ & 10.01 & 244.48 \\
\hline 636 & $04-07-88$ & 9.57 & 244.91 \\
\hline 636 & $05-05-88$ & 9.98 & 244.51 \\
\hline 636 & $06-09-88$ & 10.39 & 244.09 \\
\hline 636 & $06-29-88$ & 10.40 & 244.08 \\
\hline 636 & $07-28-88$ & 9.70 & 244.79 \\
\hline 636 & $08-31-88$ & 9.52 & 244.97 \\
\hline 636 & $10-04-88$ & 10.27 & 244.22 \\
\hline 636 & $10-04-88$ & 10.31 & 244.17 \\
\hline 636 & $10-07-88$ & 9.62 & 244.86 \\
\hline 636 & $10-24-88$ & 10.40 & 244.09 \\
\hline 636 & $11-01-88$ & 10.03 & 244.45 \\
\hline 636 & $11-01-88$ & 10.41 & 244.08 \\
\hline 636 & $11-14-88$ & 9.98 & 244.50 \\
\hline 636 & $11-28-88$ & 9.34 & 245.15 \\
\hline 636 & $12-05-88$ & 9.84 & 244.64 \\
\hline 636 & $12-06-88$ & 9.92 & 244.56 \\
\hline 636 & $12-12-88$ & 10.06 & 244.42 \\
\hline 636 & $12-20-88$ & 10.30 & 244.18 \\
\hline 636 & $12-27-88$ & 9.45 & 245.03 \\
\hline 636 & $01-02-89$ & 8.97 & 245.51 \\
\hline 636 & $01-05-89$ & 9.21 & 245.28 \\
\hline 636 & $01-11-89$ & 9.09 & 245.40 \\
\hline 636 & $01-17-89$ & 8.30 & 246.19 \\
\hline 636 & $01-27-89$ & 9.30 & 245.19 \\
\hline 636 & $01-31-89$ & 9.54 & 244.95 \\
\hline 636 & $02-08-89$ & 9.07 & 245.42 \\
\hline 636 & $02-10-89$ & 9.32 & 245.16 \\
\hline 636 & $02-17-89$ & 9.33 & 245.15 \\
\hline 636 & $02-24-89$ & 9.45 & 245.03 \\
\hline 636 & $03-03-89$ & 9.07 & 245.41 \\
\hline 636 & $03-09-89$ & 8.84 & 245.64 \\
\hline 636 & $03-15-89$ & 9.45 & 245.03 \\
\hline 636 & $03-22-89$ & 9.15 & 245.34 \\
\hline 636 & $03-28-89$ & 9.20 & 245.29 \\
\hline 636 & $04-07-89$ & 9.15 & 245.34 \\
\hline 636 & $04-14-89$ & 9.31 & 245.17 \\
\hline 636 & $04-22-89$ & 9.48 & 245.01 \\
\hline 636 & $05-04-89$ & 9.96 & 244.52 \\
\hline 636 & $05-12-89$ & 9.48 & 245.01 \\
\hline 636 & $05-17-89$ & 9.70 & 244.78 \\
\hline 636 & $05-26-89$ & 9.38 & 245.11 \\
\hline 636 & $06-01-89$ & 9.88 & 244.60 \\
\hline 636 & $06-09-89$ & 9.13 & 245.35 \\
\hline 636 & $06-16-89$ & 9.08 & 245.40 \\
\hline 636 & $06-23-89$ & 8.44 & 246.05 \\
\hline 636 & $06-28-89$ & 9.11 & 245.38 \\
\hline 636 & $07-07-89$ & 8.77 & 245.72 \\
\hline 636 & $07-14-89$ & 9.55 & 244.93 \\
\hline 636 & $07-21-89$ & 9.54 & 244.95 \\
\hline 636 & $07-28-89$ & 10.07 & 244.42 \\
\hline 636 & 08-04-89 & 9.78 & 244.70 \\
\hline 636 & $08-11-89$ & 9.76 & 244.73 \\
\hline 636 & $08-18-89$ & 9.76 & 244.73 \\
\hline 636 & $08.30-89$ & 9.17 & 245.31 \\
\hline 636 & $09-07-89$ & 9.20 & 245.29 \\
\hline 636 & $09-18-89$ & 9.15 & 245.34 \\
\hline
\end{tabular}


Table A.1 (continued)

\begin{tabular}{|c|c|c|c|}
\hline $\begin{array}{l}\text { Well } \\
\text { No. }\end{array}$ & $\begin{array}{c}\text { Date } \\
\text { measured }\end{array}$ & $\begin{array}{l}\text { Depth to } \\
\text { water }(m)\end{array}$ & $\begin{array}{l}\text { Water-level } \\
\text { elevation (m) }\end{array}$ \\
\hline 636 & $09-27-89$ & 9.22 & 245.26 \\
\hline 636 & $01-15-90$ & 9.36 & 24512 \\
\hline 636 & $02-15-90$ & 8.78 & 245.70 \\
\hline 636 & $03-14-90$ & 9.18 & 245.30 \\
\hline 636 & $04-23-90$ & 9.60 & 244.88 \\
\hline 636 & $05-21-90$ & 9.44 & 245.04 \\
\hline 636 & $06-26-90$ & 10.00 & 244.48 \\
\hline 636 & $07-25-90$ & 9.59 & 244.90 \\
\hline 636 & $08-23-90$ & 9.83 & 244.65 \\
\hline 636 & $09-25-90$ & 10.24 & 244.24 \\
\hline 640 & $01-15-88$ & 10.57 & 244.24 \\
\hline 640 & $02-04-88$ & 9.55 & 245.26 \\
\hline 640 & $03-03-88$ & 9.74 & 245.08 \\
\hline 640 & $04-08-88$ & 9.77 & 245.05 \\
\hline 640 & $05-05-88$ & 9.97 & 244.84 \\
\hline 640 & $06-09-88$ & 10.45 & 244.37 \\
\hline 640 & $06-29-88$ & 10.67 & 244.14 \\
\hline 640 & $07-28-88$ & 10.54 & 244.27 \\
\hline 640 & $08-31-88$ & 10.75 & 244.07 \\
\hline 640 & $10-04-88$ & 10.46 & 244.35 \\
\hline 640 & $10-05-88$ & 10.70 & 244.12 \\
\hline 640 & $10-24-88$ & 10.81 & 244.00 \\
\hline 640 & $11-01-88$ & 10.73 & 244.09 \\
\hline 640 & $11-01-88$ & 10.87 & 243.95 \\
\hline 640 & $11-07-88$ & 10.67 & 244.14 \\
\hline 640 & $12-06-88$ & 10.02 & 244.79 \\
\hline 640 & $12-12-88$ & 9.15 & 245.67 \\
\hline 640 & $12-20-88$ & 10.43 & 244.38 \\
\hline 640 & $12-27-98$ & 10.64 & 244.17 \\
\hline 640 & $01-02-89$ & 10.09 & 244.72 \\
\hline 640 & $01-11-89$ & 9.17 & 245.64 \\
\hline 640 & $01-17-89$ & 9.68 & 245.13 \\
\hline 640 & $04-22-89$ & 11.83 & 242.99 \\
\hline 640 & $05-04-89$ & 10.27 & 244.95 \\
\hline 640 & $05-12-89$ & 10.37 & 244.85 \\
\hline 640 & $05-17-89$ & 10.39 & 244.83 \\
\hline 640 & $05-26-89$ & 10.59 & 244.63 \\
\hline 640 & $06-01-89$ & 10.75 & 244.47 \\
\hline 640 & $06-09-89$ & 10.65 & 244.57 \\
\hline 640 & $06-16-89$ & 10.66 & 244.56 \\
\hline 640 & $06-23-89$ & 10.19 & 245.03 \\
\hline 640 & $06-28-89$ & 10.19 & 245.03 \\
\hline 640 & 07-07-89 & 10.29 & 244.93 \\
\hline 640 & $07-14-89$ & 10.35 & 244.87 \\
\hline 640 & $07-21-89$ & 10.34 & 244.88 \\
\hline 640 & $07-28-89$ & 10.53 & 244.69 \\
\hline 640 & $08-04-89$ & 10.57 & 244.65 \\
\hline 640 & $08-11-89$ & 10.54 & 244.68 \\
\hline 640 & $08-18-89$ & 10.52 & 244.70 \\
\hline 640 & $08-30-89$ & 10.71 & 244.51 \\
\hline 640 & $09-07-89$ & 10.67 & 244.55 \\
\hline 640 & $09-18-89$ & 10.63 & 244.59 \\
\hline 640 & $09-27-89$ & 10.68 & 244.54 \\
\hline 640 & $01-15-90$ & 10.17 & 245.05 \\
\hline 640 & $02-15-90$ & 9.70 & 245.52 \\
\hline 640 & $03-14-90$ & 989 & $2 \times 5.33$ \\
\hline 640 & $04-23-90$ & 10.35 & 244.87 \\
\hline 640 & $05-21-90$ & Blocked & \\
\hline
\end{tabular}


Table A.1 (continued)

\begin{tabular}{|c|c|c|c|}
\hline $\begin{array}{l}\text { Well } \\
\text { No. }\end{array}$ & $\begin{array}{c}\text { Date } \\
\text { measured }\end{array}$ & $\begin{array}{l}\text { Depth to } \\
\text { water }(\mathrm{m})\end{array}$ & $\begin{array}{l}\text { Water-level } \\
\text { elevation }(\mathrm{m})\end{array}$ \\
\hline 640 & $06-26-90$ & Blocked & \\
\hline 640 & $07-25-90$ & Blocked & \\
\hline 640 & $08-23-90$ & Blocked & \\
\hline 640 & $09-25-90$ & Blocked & \\
\hline 642 & $01-15-88$ & 2.46 & 234.41 \\
\hline 642 & $02-04-88$ & 1.88 & 235.00 \\
\hline 642 & $03-03-88$ & 2.15 & 234.73 \\
\hline 642 & $04-08-88$ & 2.23 & 234.65 \\
\hline 642 & $05-05-88$ & 2.42 & 234.46 \\
\hline 642 & $06-09-88$ & 2.87 & 234.01 \\
\hline 642 & $06-29-88$ & 1.92 & 234.96 \\
\hline 642 & $07-28-88$ & 2.45 & 234.43 \\
\hline 642 & $08-31-88$ & 3.06 & 233.82 \\
\hline 642 & $10-05-88$ & 2.92 & 233.95 \\
\hline 642 & $10-24-88$ & 3.05 & 233.83 \\
\hline 642 & $11-01-88$ & 3.08 & 233.80 \\
\hline 642 & $11-01-88$ & 2.99 & 233.89 \\
\hline 642 & $11-07-88$ & 2.44 & 234.44 \\
\hline 642 & $11-14-88$ & 2.49 & 234.39 \\
\hline 642 & $11-21-88$ & 2.07 & 234.81 \\
\hline 642 & $11-28-88$ & 2.06 & 234.82 \\
\hline 642 & $12-05-88$ & 2.11 & 234.77 \\
\hline 642 & $12-06-88$ & 2.10 & 234.78 \\
\hline 642 & $12-12-88$ & 2.22 & 234.66 \\
\hline 642 & $12-20-88$ & 2.38 & 234.50 \\
\hline 642 & $12-27-88$ & 1.96 & 234.92 \\
\hline 642 & $01-02-89$ & 1.73 & 235.15 \\
\hline 642 & $01-06-89$ & 1.66 & 235.22 \\
\hline 642 & $01-11-89$ & 1.61 & 235.27 \\
\hline 642 & $01-17-89$ & 1.24 & 235.64 \\
\hline 642 & $01-27-89$ & 1.49 & 235.39 \\
\hline 642 & $01-31-89$ & 1.51 & 235.37 \\
\hline 642 & $02-08-89$ & 1.50 & 235.38 \\
\hline 642 & $02-10-89$ & 1.51 & 235.37 \\
\hline 642 & $02-17-89$ & 1.47 & 235.41 \\
\hline 642 & $02-24-89$ & 1.42 & 235.45 \\
\hline 642 & $03-03-89$ & 1.42 & 235.45 \\
\hline 642 & $03-09-89$ & 1.46 & 235.41 \\
\hline 642 & $03-15-89$ & 1.50 & 235.38 \\
\hline 642 & $03-22-89$ & 1.50 & 235.38 \\
\hline 642 & $03-28-89$ & 1.54 & 235.34 \\
\hline 642 & $04-07-89$ & 1.48 & 235.39 \\
\hline 642 & $04-07-89$ & 1.50 & 235.38 \\
\hline 642 & $04-14-89$ & 1.52 & 235.36 \\
\hline 642 & $04-22-89$ & 1.72 & 235.16 \\
\hline 642 & $05-04-89$ & 1.94 & 234.94 \\
\hline 642 & $05-08-89$ & 1.77 & 235.10 \\
\hline 642 & $05-12-89$ & 1.78 & 235.09 \\
\hline 642 & $05-17-89$ & 1.83 & 235.05 \\
\hline 642 & $05-26-89$ & 1.91 & 234.97 \\
\hline 642 & $06-01-89$ & 1.96 & 234.92 \\
\hline 642 & $06-08-89$ & 1.90 & 234.98 \\
\hline 642 & $06-09-89$ & 1.74 & 235.14 \\
\hline 642 & $06-16-89$ & 1.73 & 235.15 \\
\hline 642 & $06-23-89$ & 1.30 & 235.58 \\
\hline 642 & $06-28-89$ & 1.40 & 235.48 \\
\hline 642 & $07-07-89$ & 1.24 & 235.63 \\
\hline 642 & $07-10-89$ & 1.33 & 235.55 \\
\hline
\end{tabular}


Table A.1 (continued)

\begin{tabular}{|c|c|c|c|}
\hline $\begin{array}{l}\text { Well } \\
\text { No. }\end{array}$ & $\begin{array}{c}\text { Date } \\
\text { measured }\end{array}$ & $\begin{array}{l}\text { Depth to } \\
\text { water (m) }\end{array}$ & $\begin{array}{l}\text { Water-level } \\
\text { elevation }(\mathrm{m})\end{array}$ \\
\hline 642 & $07-14-89$ & 1.42 & 235.45 \\
\hline 642 & $07-21-89$ & 1.41 & 235.47 \\
\hline 642 & $07-28-89$ & 1.74 & 235.14 \\
\hline 642 & $08-03-89$ & 1.80 & 235.08 \\
\hline 642 & $08-04-89$ & 1.83 & 235.05 \\
\hline 642 & $08-11-89$ & 1.75 & 235.13 \\
\hline 642 & $08-18-89$ & 1.73 & 235.15 \\
\hline 642 & $08-30-89$ & 1.77 & 235.11 \\
\hline 642 & $09-07-89$ & 1.73 & 235.15 \\
\hline 642 & $09-18-89$ & 1.68 & 235.20 \\
\hline 642 & $09-20-89$ & 2.02 & 234.86 \\
\hline 642 & $09-27-89$ & 1.74 & 235.13 \\
\hline 642 & $10-18-89$ & 1.38 & 235.50 \\
\hline 642 & $01-15-90$ & 1.54 & 235.34 \\
\hline 642 & $02-15-90$ & 1.13 & 235.75 \\
\hline 642 & $03-14-90$ & 1.43 & 235.45 \\
\hline 642 & $04-23-90$ & 1.95 & 234.93 \\
\hline 642 & $05-21-90$ & 1.83 & 235.05 \\
\hline 642 & $06-26-90$ & 2.10 & 234.78 \\
\hline 642 & $07-25-90$ & 1.94 & 234.94 \\
\hline 642 & $08-23-90$ & 2.09 & 234.79 \\
\hline 642 & $09-25-90$ & 2.42 & 234.45 \\
\hline 645 & $01-12-88$ & 2.66 & 230.97 \\
\hline 645 & $02-04-88$ & 2.24 & 231.39 \\
\hline 645 & $03-03-88$ & 2.48 & 231.15 \\
\hline 645 & $04-08-88$ & 2.38 & 231.25 \\
\hline 645 & $05-05-88$ & 2.46 & 231.17 \\
\hline 645 & $06-09-88$ & 2.60 & 231.03 \\
\hline 645 & $06-29-88$ & 2.66 & 230.97 \\
\hline 645 & $07-28-88$ & 2.63 & 231.00 \\
\hline 645 & $08-31-88$ & 2.73 & 230.90 \\
\hline 645 & $10-04-88$ & 2.66 & 230.97 \\
\hline 645 & $10-05-88$ & 2.67 & 230.96 \\
\hline 645 & $10-24-88$ & 2.70 & 230.93 \\
\hline 645 & $11-01-88$ & 2.66 & 230.97 \\
\hline 645 & $11-01-88$ & 2.67 & 230.96 \\
\hline 645 & $11-07-88$ & 2.59 & 231.04 \\
\hline 645 & $11-14-88$ & 2.62 & 231.01 \\
\hline 645 & $11-28-88$ & 2.53 & 231.10 \\
\hline 645 & $12-05-88$ & 2.55 & 231.08 \\
\hline 645 & $12-06-88$ & 2.59 & 231.05 \\
\hline 645 & $12-12-88$ & 2.58 & 231.05 \\
\hline 645 & $12-20-88$ & 2.55 & 231.08 \\
\hline 645 & $12-27-88$ & 2.50 & 231.13 \\
\hline 645 & $01-02-89$ & 2.20 & 231.43 \\
\hline 645 & $01-11-89$ & 2.26 & 231.37 \\
\hline 645 & $01-11-89$ & 2.29 & 231.34 \\
\hline 645 & $01-17-89$ & 2.12 & 231.51 \\
\hline 645 & $01-27-89$ & 2.29 & 231.34 \\
\hline 645 & $01-31-89$ & 2.27 & 231.36 \\
\hline 645 & $02-08-89$ & 2.05 & 231.58 \\
\hline 645 & $02-10-89$ & 2.13 & 231.50 \\
\hline 645 & $02-17-89$ & 2.07 & 231.56 \\
\hline 645 & $02-24-89$ & 2.16 & 231.47 \\
\hline 645 & $03-03-89$ & 2.07 & 231.56 \\
\hline 645 & 03-09-89 & 2.02 & 231.61 \\
\hline 645 & $03-15-89$ & 2.25 & 231.38 \\
\hline 645 & $03-22-89$ & 1.99 & 231.64 \\
\hline
\end{tabular}


Table A.1 (continued)

\begin{tabular}{|c|c|c|c|}
\hline $\begin{array}{l}\text { Well } \\
\text { No. }\end{array}$ & $\begin{array}{c}\text { Date } \\
\text { measured }\end{array}$ & $\begin{array}{l}\text { Depth to } \\
\text { water (m) }\end{array}$ & $\begin{array}{l}\text { Water-level } \\
\text { elevation }(\mathrm{m})\end{array}$ \\
\hline 645 & $03-28-89$ & 2.16 & 231.47 \\
\hline 645 & $04-07-89$ & 2.13 & 231.50 \\
\hline 645 & $04-14-89$ & 1.78 & 231.85 \\
\hline 645 & $04-22-89$ & 2.44 & 231.19 \\
\hline 645 & $05-04-89$ & 2.38 & 231.26 \\
\hline 645 & $05-12-89$ & 2.22 & 231.41 \\
\hline 645 & $05-17-89$ & 2.31 & 231.32 \\
\hline 645 & $05-26-89$ & 2.35 & 231.28 \\
\hline 645 & $06-01-89$ & 2.31 & 231.32 \\
\hline 645 & $06-09-89$ & 1.78 & 231.85 \\
\hline 645 & $06-16-89$ & 1.73 & 231.90 \\
\hline 645 & $06-23-89$ & 1.92 & 231.71 \\
\hline 645 & $06-28-89$ & 2.21 & 231.42 \\
\hline 645 & $07-07-89$ & 1.89 & 231.74 \\
\hline 645 & $07-14-89$ & 2.34 & 231.29 \\
\hline 645 & $07-21-89$ & 2.31 & 231.32 \\
\hline 645 & $07-28-89$ & 2.28 & 231.35 \\
\hline 645 & $08-04-89$ & 2.26 & 231.37 \\
\hline 645 & $08-11-89$ & 2.27 & 231.36 \\
\hline 645 & $08-18-89$ & 2.26 & 231.37 \\
\hline 645 & $08-30-89$ & 2.33 & 231.30 \\
\hline 645 & 09-07-89 & 2.29 & 231.34 \\
\hline 645 & 09-18-89 & 2.29 & 231.34 \\
\hline 645 & $09-27-89$ & 2.30 & 231.33 \\
\hline 645 & $01-15-90$ & 1.99 & 231.64 \\
\hline 645 & $03-14-90$ & 2.13 & 231.50 \\
\hline 645 & $04-23-90$ & 2.17 & 231.46 \\
\hline 645 & $05-21-90$ & 2.15 & 231.48 \\
\hline 645 & $06-26-90$ & 2.39 & 231.24 \\
\hline 645 & $07-25-90$ & 2.25 & 231.38 \\
\hline 645 & $08-23-90$ & 2.20 & 231.44 \\
\hline 645 & $09-25-90$ & 2.38 & 231.25 \\
\hline 646 & $01-04-88$ & 4.59 & 235.32 \\
\hline 646 & $01-08-88$ & 4.73 & 235.18 \\
\hline 646 & $01-12-88$ & 4.91 & 235.00 \\
\hline 646 & $01-14-88$ & 4.85 & 235.06 \\
\hline 646 & $01-21-88$ & 4.23 & 235.68 \\
\hline 646 & $01-22-88$ & 4.30 & 235.61 \\
\hline 646 & $01-25-88$ & 4.36 & 235.55 \\
\hline 646 & $01-28-88$ & 4.48 & 235.43 \\
\hline 646 & $02-01-88$ & 4.53 & 235.38 \\
\hline 646 & $02-04-88$ & 4.26 & 235.65 \\
\hline 646 & $02-11-88$ & 4.33 & 235.58 \\
\hline 646 & $02-18-88$ & 4.29 & 235.62 \\
\hline 646 & $02-26-88$ & 4.40 & 235.51 \\
\hline 646 & $03-03-88$ & 4.46 & 235.45 \\
\hline 646 & $03-03-88$ & 4.41 & 235.50 \\
\hline 646 & $03-04-88$ & 4.45 & 235.46 \\
\hline 646 & $03-05-88$ & 4.47 & 235.44 \\
\hline 646 & $03-06-88$ & 4.48 & 235.43 \\
\hline 646 & $03-07-88$ & 4.50 & 235.41 \\
\hline 646 & $03-08-88$ & 4.48 & 235.43 \\
\hline 646 & $03-09-88$ & 4.49 & 235.42 \\
\hline 646 & $03-09-88$ & 4.46 & 235.45 \\
\hline 646 & $03-10-88$ & 4.19 & 235.73 \\
\hline 646 & $03-11-88$ & 4.10 & 235.81 \\
\hline 646 & $03-12-88$ & 4.14 & 235.77 \\
\hline 646 & $03-13-88$ & 4.11 & 235.80 \\
\hline
\end{tabular}


Table A.1 (continued)

\begin{tabular}{|c|c|c|c|}
\hline $\begin{array}{l}\text { Well } \\
\text { No. }\end{array}$ & $\begin{array}{c}\text { Date } \\
\text { measured }\end{array}$ & $\begin{array}{l}\text { Depth to } \\
\text { water }(\mathrm{m})\end{array}$ & $\begin{array}{l}\text { Water-level } \\
\text { elevation }(\mathrm{m})\end{array}$ \\
\hline 646 & $03-14-88$ & 4.08 & 235.83 \\
\hline 646 & $03-15-88$ & 4.15 & 235.77 \\
\hline 646 & $03-16-88$ & 4.17 & 235.74 \\
\hline 646 & $03-16-88$ & 4.19 & 235.73 \\
\hline 646 & $03-17-88$ & 4.20 & 235.71 \\
\hline 646 & $03-18-88$ & 4.22 & 235.70 \\
\hline 646 & $03-19-88$ & 4.23 & 235.68 \\
\hline 646 & $03-20-88$ & 4.26 & 235.65 \\
\hline 646 & $03-21-88$ & 4.28 & 235.63 \\
\hline 646 & $03-22-88$ & 4.30 & 235.61 \\
\hline 646 & $03-23-88$ & 4.31 & 235.60 \\
\hline 646 & $03-24-88$ & 4.32 & 235.59 \\
\hline 646 & $03-25-88$ & 4.23 & 235.69 \\
\hline 646 & $03-26-88$ & 4.20 & 235.71 \\
\hline 646 & $03-27-88$ & 4.19 & 235.72 \\
\hline 646 & $03-28-88$ & 4.18 & 235.73 \\
\hline 646 & $03-28-88$ & 4.20 & 235.72 \\
\hline 646 & $03-29-88$ & 4.17 & 235.74 \\
\hline 646 & $03-30-88$ & 4.20 & 235.71 \\
\hline 646 & $03-31-88$ & 4.19 & 235.72 \\
\hline 646 & $04-01-88$ & 4.21 & 235.70 \\
\hline 646 & $04-02-88$ & 4.13 & 235.78 \\
\hline 646 & $04-03-88$ & 4.17 & 235.74 \\
\hline 646 & $04-04-88$ & 4.10 & 235.81 \\
\hline 646 & $04-04-88$ & 4.09 & 235.82 \\
\hline 646 & $04-05-88$ & 4.11 & 235.80 \\
\hline 646 & $04-06-88$ & 4.02 & 235.90 \\
\hline 646 & $04-07-88$ & 3.95 & 235.96 \\
\hline 646 & $04-08-88$ & 3.94 & 235.97 \\
\hline 646 & 04-08-88 & 3.98 & 235.94 \\
\hline 646 & $04-09-88$ & 4.00 & 235.91 \\
\hline 646 & $04-10-88$ & 3.98 & 235.93 \\
\hline 646 & $04-11-88$ & 3.98 & 235.94 \\
\hline 646 & $04-12-88$ & 3.98 & 235.93 \\
\hline 646 & $04-13-88$ & 3.98 & 235.93 \\
\hline 646 & $04-14-88$ & 4.01 & 235.90 \\
\hline 646 & $04-15-88$ & 4.04 & 235.87 \\
\hline 646 & $04-16-88$ & 4.04 & 235.88 \\
\hline 646 & $04-17-88$ & 4.05 & 235.87 \\
\hline 646 & $04-18-88$ & 3.98 & 235.93 \\
\hline 646 & $04-19-88$ & 3.87 & 236.04 \\
\hline 646 & $04-20-88$ & 3.85 & 236.06 \\
\hline 646 & $04-21-88$ & 3.83 & 236.09 \\
\hline 646 & $04-22-88$ & 3.85 & 236.06 \\
\hline 646 & $04-23-88$ & 3.88 & 236.03 \\
\hline 646 & $04-24-88$ & 3.91 & 236.00 \\
\hline 646 & $04-25-88$ & 3.93 & 235.98 \\
\hline 646 & $04-26-88$ & 3.95 & 235.96 \\
\hline 646 & $04-27-88$ & 3.98 & 235.93 \\
\hline 646 & $04-28-88$ & 4.02 & 235.89 \\
\hline 646 & $04-29-88$ & 4.03 & 235.88 \\
\hline 646 & $04-30-88$ & 4.06 & 235.85 \\
\hline 646 & $05-01-88$ & 4.08 & 235.83 \\
\hline 646 & $05-02-88$ & 4.10 & 235.81 \\
\hline 646 & $05-03-88$ & 4.10 & 235.81 \\
\hline 646 & $05-03-88$ & 4.12 & 235.79 \\
\hline 646 & $05-04-88$ & 4.08 & 235.83 \\
\hline 646 & $05-05-88$ & 4.11 & 235.80 \\
\hline
\end{tabular}


Table A.1 (continued)

\begin{tabular}{|c|c|c|c|}
\hline $\begin{array}{l}\text { Well } \\
\text { No. }\end{array}$ & $\begin{array}{c}\text { Date } \\
\text { measured }\end{array}$ & $\begin{array}{l}\text { Depth to } \\
\text { water (m) }\end{array}$ & $\begin{array}{l}\text { Water-level } \\
\text { elevation }(\mathrm{m})\end{array}$ \\
\hline 646 & $05-05-88$ & 3.79 & 236.12 \\
\hline 646 & $05-06-88$ & 4.15 & 235.77 \\
\hline 646 & $05-07-88$ & 4.17 & 235.74 \\
\hline 646 & $05-08-88$ & 4.16 & 235.75 \\
\hline 646 & $05-09-88$ & 4.10 & 235.81 \\
\hline 646 & $05-10-88$ & 4.10 & 235.81 \\
\hline 646 & $05-11-88$ & 4.16 & 235.75 \\
\hline 646 & $05-12-88$ & 4.14 & 235.77 \\
\hline 646 & $05-12-88$ & 4.20 & 235.71 \\
\hline 646 & $05-13-88$ & 4.22 & 235.69 \\
\hline 646 & $05-14-88$ & 4.24 & 235.67 \\
\hline 646 & $05-15-88$ & 4.26 & 235.66 \\
\hline 646 & $05-16-88$ & 4.27 & 235.64 \\
\hline 646 & $05-17-88$ & 4.31 & 235.60 \\
\hline 646 & $05-18-88$ & 4.33 & 235.58 \\
\hline 646 & $05-19-88$ & 4.33 & 235.58 \\
\hline 646 & $05-20-88$ & 4.37 & 235.54 \\
\hline 646 & $05-20-88$ & 4.35 & 235.56 \\
\hline 646 & $05-21-88$ & 4.40 & 235.51 \\
\hline 646 & $05-22-88$ & 4.41 & 235.50 \\
\hline 646 & $05-23-88$ & 4.43 & 235.48 \\
\hline 646 & $05-24-88$ & 4.41 & 235.50 \\
\hline 646 & $05-25-88$ & 4.28 & 235.63 \\
\hline 646 & $05-26-88$ & 4.46 & 235.45 \\
\hline 646 & $05-26-88$ & 4.44 & 235.47 \\
\hline 646 & $05-27-88$ & 4.49 & 235.42 \\
\hline 646 & $05-28-88$ & 4.51 & 235.40 \\
\hline 646 & $05-29-88$ & 4.52 & 235.39 \\
\hline 646 & $05-30-88$ & 4.54 & 235.37 \\
\hline 646 & $05-31-88$ & 4.54 & 235.37 \\
\hline 646 & $06-01-88$ & 4.55 & 235.37 \\
\hline 646 & $06-02-88$ & 4.50 & 235.41 \\
\hline 646 & $06-02-88$ & 4.55 & 235.36 \\
\hline 646 & $06-03-88$ & 4.57 & 235.34 \\
\hline 646 & $06-04-88$ & 4.59 & 235.32 \\
\hline 646 & $06-05-88$ & 4.61 & 235.30 \\
\hline 646 & $06-06-88$ & 4.62 & 235.29 \\
\hline 646 & $06-07-88$ & 4.63 & 235.28 \\
\hline 646 & $06-08-88$ & 4.64 & 235.27 \\
\hline 646 & $06-09-88$ & 4.64 & 235.27 \\
\hline 646 & 06-09-88 & 4.60 & 235.31 \\
\hline 646 & $06-09-88$ & 4.63 & 235.28 \\
\hline 646 & $06-10-88$ & 4.57 & 235.34 \\
\hline 646 & $06-11-88$ & 4.63 & 235.29 \\
\hline 646 & $06-12-88$ & 4.64 & 235.27 \\
\hline 646 & $06-13-88$ & 4.66 & 235.26 \\
\hline 646 & $06-14-88$ & 4.69 & 235.22 \\
\hline 646 & $06-15-88$ & 4.71 & 235.20 \\
\hline 646 & $06-16-88$ & 4.72 & 235.19 \\
\hline 646 & $06-17-88$ & 4.73 & 235.18 \\
\hline 646 & $06-18-88$ & 4.82 & 235.09 \\
\hline 646 & $06-19-88$ & 4.83 & 235.09 \\
\hline 646 & $06-20-88$ & 4.82 & 235.09 \\
\hline 646 & $06-21-88$ & 4.77 & 235.14 \\
\hline 646 & $06-29-88$ & 4.73 & 235.19 \\
\hline 646 & $07-28-88$ & 4.51 & 235.40 \\
\hline 646 & $08-31-88$ & 4.87 & 235.05 \\
\hline 646 & $10-04-88$ & 4.11 & 235.80 \\
\hline
\end{tabular}


Table A.1 (continued)

\begin{tabular}{|c|c|c|c|}
\hline $\begin{array}{l}\text { Well } \\
\text { No. }\end{array}$ & $\begin{array}{c}\text { Date } \\
\text { measured }\end{array}$ & $\begin{array}{l}\text { Depth to } \\
\text { water }(m)\end{array}$ & $\begin{array}{l}\text { Water-level } \\
\text { clevation }(\mathrm{m})\end{array}$ \\
\hline 646 & $10-05-88$ & 4.15 & 235.77 \\
\hline 646 & $10-24-88$ & 4.17 & 235.74 \\
\hline 646 & $11-01-88$ & 4.48 & 235.43 \\
\hline 646 & $11-01-88$ & 4.23 & 235.68 \\
\hline 646 & $11-07-88$ & 3.55 & 236.37 \\
\hline 646 & $11-14-88$ & 3.72 & 236.19 \\
\hline 646 & $11-21-88$ & 2.71 & 237.20 \\
\hline 646 & $11-28-88$ & 2.64 & 237.27 \\
\hline 646 & $12-05-88$ & 3.13 & 236.78 \\
\hline 646 & $12-06-88$ & 3.21 & 236.70 \\
\hline 646 & $12-12-88$ & 3.41 & 236.51 \\
\hline 646 & $12-20-88$ & 2.71 & 237.20 \\
\hline 646 & $12-27-88$ & 2.66 & 237.26 \\
\hline 646 & $01-02-89$ & 2.41 & 237.50 \\
\hline 646 & $01-11-89$ & 2.28 & 237.63 \\
\hline 646 & $01-11-89$ & 2.32 & 237.59 \\
\hline 646 & $01-17-89$ & 2.13 & 237.78 \\
\hline 646 & $01-27-89$ & 2.52 & 237.40 \\
\hline 646 & $01-31-89$ & 2.49 & 237.42 \\
\hline 646 & $02-08-89$ & 2.20 & 237.72 \\
\hline 646 & $02-10-89$ & 2.22 & 237.69 \\
\hline 646 & $02-17-89$ & 1.83 & 238.08 \\
\hline 646 & $02-24-89$ & 1.92 & 237.99 \\
\hline 646 & $03-03-89$ & 2.05 & 237.87 \\
\hline 646 & $03-09-89$ & 2.07 & 237.84 \\
\hline 646 & $03-15-89$ & 2.26 & 237.66 \\
\hline 646 & $03-22-89$ & 2.06 & 237.835 \\
\hline 646 & $03-28-89$ & 2.28 & 237.63 \\
\hline 646 & $04-07-89$ & 2.27 & 237.65 \\
\hline 646 & $04-22-89$ & 4.70 & 235.21 \\
\hline 646 & $05-04-89$ & 4.57 & 235.34 \\
\hline 646 & $05-12-89$ & 4.26 & 235.66 \\
\hline 646 & $05-17-89$ & 2.84 & 237.07 \\
\hline 646 & $05-26-89$ & 4.42 & 235.49 \\
\hline 646 & $06-01-89$ & 4.41 & 235.50 \\
\hline 646 & $06-09-89$ & 4.26 & 235.66 \\
\hline 646 & $06-16-89$ & 4.22 & 235.69 \\
\hline 646 & $06-23-89$ & 3.84 & 236.08 \\
\hline 646 & $06-28-89$ & 4.12 & 235.80 \\
\hline 646 & $07-07-89$ & 4.03 & 235.88 \\
\hline 646 & $07-14-89$ & 4.46 & 235.45 \\
\hline 646 & $07-21-89$ & 4.42 & 235.49 \\
\hline 646 & $07-28-89$ & 4.36 & 235.55 \\
\hline 646 & $08-04-89$ & 4.32 & 235.59 \\
\hline 646 & $08-11-89$ & 4.28 & 235.63 \\
\hline 646 & $08-18-89$ & 4.27 & 235.64 \\
\hline 646 & $08-30-89$ & 4.79 & 235.12 \\
\hline 646 & $09-07-89$ & 4.78 & 235.13 \\
\hline 646 & $09-18-89$ & 4.67 & 235.24 \\
\hline 646 & $09-27-89$ & 4.80 & 235.12 \\
\hline 646 & $01-15-90$ & 4.50 & 235.41 \\
\hline 646 & $03-14-90$ & 4.39 & 235.52 \\
\hline 646 & $04-23-90$ & 4.61 & 235.30 \\
\hline 646 & $05-21-90$ & 4.49 & 235.42 \\
\hline 646 & $06-26-90$ & 4.73 & 235.19 \\
\hline 646 & $07-25-90$ & 4.56 & 235.35 \\
\hline 6046 & $08-23-90$ & 4.59 & 235.32 \\
\hline 646 & $09.25-90$ & 4.86 & 235.05 \\
\hline
\end{tabular}


Table A.1 (continued)

\begin{tabular}{|c|c|c|c|}
\hline $\begin{array}{l}\text { Nell } \\
\text { No. }\end{array}$ & $\begin{array}{c}\text { Date } \\
\text { measured }\end{array}$ & $\begin{array}{l}\text { Depth to } \\
\text { water }(m)\end{array}$ & $\begin{array}{l}\text { Water-lcvel } \\
\text { elcvation }(\mathrm{m})\end{array}$ \\
\hline 647 & $10-24-88$ & 10.35 & 239.75 \\
\hline 647 & $11-01-88$ & 10.37 & 239.74 \\
\hline 647 & 11.07 .88 & 10.30 & 239.81 \\
\hline 647 & $11-14-88$ & 10.27 & 239.84 \\
\hline 647 & $12-06-88$ & 10.21 & 239.90 \\
\hline 647 & $12-12-88$ & 10.38 & 239.72 \\
\hline 647 & $12-20-88$ & 10.22 & 239.88 \\
\hline 647 & $12-27-88$ & 10.14 & 239.96 \\
\hline 647 & $01-02-89$ & 9.99 & 240.11 \\
\hline 647 & $01-11-89$ & 9.93 & 240.17 \\
\hline 647 & $01-17-89$ & 9.76 & 240.35 \\
\hline 647 & $01-27-89$ & 10.02 & 240.08 \\
\hline 647 & $01-31-89$ & 9.94 & 240.16 \\
\hline 647 & $02-10-89$ & 9.82 & 240.29 \\
\hline 647 & $02-17-89$ & 9.88 & 240.22 \\
\hline 647 & $02-24-89$ & 10.07 & 240.03 \\
\hline 647 & 03-03-89 & 9.80 & 240.31 \\
\hline 647 & $03-15-89$ & 9.78 & 240.33 \\
\hline 647 & $03-22-89$ & 9.70 & 240.40 \\
\hline 647 & $03-28-89$ & 9.83 & 240.27 \\
\hline 647 & $04-07-89$ & 9.78 & 240.33 \\
\hline 647 & $04-14-89$ & 10.00 & 240.09 \\
\hline 647 & $04-22-89$ & 9.95 & 240.14 \\
\hline 647 & $05-04-89$ & 10.06 & 240.03 \\
\hline 647 & $05-12-89$ & 9.86 & 240.23 \\
\hline 647 & $05-17-89$ & 9.99 & 240.10 \\
\hline 647 & $05-26-89$ & 9.97 & 240.12 \\
\hline 647 & $06-01-89$ & 10.16 & 239.93 \\
\hline 647 & 06-09-89 & 9.46 & 240.63 \\
\hline 647 & $06-16-89$ & 9.39 & 240.70 \\
\hline 647 & $06-23-89$ & 9.66 & 240.44 \\
\hline 647 & $06-28-89$ & 9.82 & 240.27 \\
\hline 647 & $07-07-89$ & 9.72 & 240.37 \\
\hline 647 & $07-14-89$ & 9.91 & 240.18 \\
\hline 647 & $07-21.89$ & 9.81 & 240.28 \\
\hline 647 & $07-28-89$ & 9.76 & 240.34 \\
\hline 647 & $08-04-89$ & 10.07 & 240.02 \\
\hline 647 & $08-11-89$ & 10.06 & 240.03 \\
\hline 647 & $08-18-89$ & 10.09 & 240.01 \\
\hline 647 & $08-30-89$ & 9.90 & 240.20 \\
\hline 647 & $09-07-89$ & 9.91 & 240.18 \\
\hline 647 & $09-18-89$ & 9.91 & 240.18 \\
\hline 647 & $09-27-89$ & 9.92 & 240.17 \\
\hline 647 & $01-15-90$ & 9.93 & 240.16 \\
\hline 647 & $02-15-90$ & 9.71 & 240.38 \\
\hline 647 & $03-14-90$ & 9.81 & 240.28 \\
\hline 647 & $04-23-90$ & 9.94 & 240.15 \\
\hline 647 & U5-21-90 & 9.92 & 240.17 \\
\hline 647 & $06-26-90$ & 10.02 & 240.08 \\
\hline 647 & $07-25-90$ & 10.00 & 240.09 \\
\hline 647 & $08-23-90$ & 10.03 & 240.06 \\
\hline 647 & $09-25-90$ & 10.12 & 239.97 \\
\hline 648 & $10-04-88$ & 8.63 & 242.82 \\
\hline 648 & $10-24-88$ & 9.24 & 242.20 \\
\hline 648 & $11-01-88$ & 9.17 & 242.27 \\
\hline 648 & $11-07-88$ & 8.87 & 242.58 \\
\hline 648 & $11-14-88$ & 8.94 & 242.50 \\
\hline 648 & $12-06-88$ & $\begin{array}{l}.944 \\
8.70\end{array}$ & 242.74 \\
\hline
\end{tabular}


Table A.1 (continued)

\begin{tabular}{|c|c|c|c|}
\hline $\begin{array}{l}\text { Well } \\
\text { No. }\end{array}$ & $\begin{array}{c}\text { Date } \\
\text { measured }\end{array}$ & $\begin{array}{l}\text { Depth to } \\
\text { water }(m)\end{array}$ & $\begin{array}{l}\text { Water-level } \\
\text { elevation }(\mathrm{m})\end{array}$ \\
\hline 648 & $12-12-88$ & 8.75 & 242.69 \\
\hline 648 & $12-20-88$ & 8.89 & 242.55 \\
\hline 648 & $12-27-88$ & 8.35 & 243.09 \\
\hline 648 & $01-02-89$ & 8.01 & 243.43 \\
\hline 648 & $01-11-89$ & 8.05 & 243.39 \\
\hline 648 & $01-17-89$ & 7.62 & 243.82 \\
\hline 648 & $01-27-89$ & 7.76 & 243.68 \\
\hline 648 & $01-31-89$ & 7.95 & 243.49 \\
\hline 648 & $02-10-89$ & 7.80 & 243.64 \\
\hline 648 & $02-17-89$ & 6.92 & 244.52 \\
\hline 648 & $02-24-89$ & 7.23 & 244.21 \\
\hline 648 & $03-03-89$ & 7.49 & 243.95 \\
\hline 648 & $03-15-89$ & 7.62 & 243.82 \\
\hline 648 & $03-22-89$ & 7.51 & 243.93 \\
\hline 648 & $03-28-89$ & 7.62 & 243.82 \\
\hline 648 & 04-07-89 & 7.62 & 243.82 \\
\hline 648 & $04-14-89$ & 7.32 & 244.13 \\
\hline 648 & $04-22-89$ & 8.03 & 243.41 \\
\hline 648 & $05-04-89$ & 8.00 & 243.44 \\
\hline 648 & $05-12-89$ & 7.62 & 243.82 \\
\hline 648 & $05-17-89$ & 7.93 & 243.52 \\
\hline 648 & $05-26-89$ & 7.84 & 243.60 \\
\hline 648 & 06-01-89 & 7.90 & 243.54 \\
\hline 648 & $06-09-89$ & 7.32 & 244.13 \\
\hline 648 & $06-16-89$ & 7.30 & 244.14 \\
\hline 648 & $06-23-89$ & 6.92 & 244.52 \\
\hline 648 & $06-28-89$ & 7.37 & 244.07 \\
\hline 648 & 07-07-89 & 7.04 & 244.41 \\
\hline 648 & $07-14-89$ & 6.96 & 244.48 \\
\hline 648 & $07-21-89$ & 6.94 & 244.50 \\
\hline 648 & $07-28-89$ & 7.75 & 243.69 \\
\hline 648 & $08-04-89$ & 7.70 & 243.74 \\
\hline 648 & $08-11-89$ & 7.70 & 243.74 \\
\hline 648 & $08-18-89$ & 7.70 & 243.74 \\
\hline 648 & $08-30-89$ & 7.52 & 243.92 \\
\hline 648 & 09-07-89 & 7.52 & 243.92 \\
\hline 648 & $09-18-89$ & 7.55 & 243.90 \\
\hline 648 & $09-27-89$ & 7.57 & 243.87 \\
\hline 648 & $01-15-90$ & 7.71 & 243.73 \\
\hline 648 & $02-15-90$ & 7.23 & 244.21 \\
\hline 648 & $03-14-90$ & 7.40 & 244.04 \\
\hline 648 & $04-23-90$ & 7.74 & 243.70 \\
\hline 648 & $05-21-90$ & 7.68 & 243.76 \\
\hline 648 & $06-26-90$ & 7.92 & 243.52 \\
\hline 648 & $07-25-90$ & 7.77 & 243.67 \\
\hline 648 & $08-23-90$ & 7.91 & 243.53 \\
\hline 648 & $09-25-90$ & 8.16 & 243.29 \\
\hline 649 & $02-04-88$ & 8.32 & 242.11 \\
\hline 649 & $03-03-88$ & 8.29 & 242.13 \\
\hline 649 & $04-08-88$ & 8.21 & 242.21 \\
\hline 649 & $05-05-88$ & 8.30 & 242.13 \\
\hline 649 & $06.09-88$ & 8.43 & 242.00 \\
\hline 649 & $06-29-88$ & 8.49 & 241.94 \\
\hline 649 & $10-04-88$ & 8.54 & 241.89 \\
\hline 649 & $10-24-88$ & 8.54 & 241.89 \\
\hline 649 & $11-28-88$ & 8.21 & 242.22 \\
\hline 649 & $12-06-88$ & 8.23 & 242.20 \\
\hline 649 & $12-27-88$ & 8.19 & 242.24 \\
\hline
\end{tabular}


Table A.1 (continued)

\begin{tabular}{|c|c|c|c|}
\hline $\begin{array}{l}\text { Well } \\
\text { No. }\end{array}$ & $\begin{array}{c}\text { Date } \\
\text { measured }\end{array}$ & $\begin{array}{l}\text { Depth to } \\
\text { water }(m)\end{array}$ & $\begin{array}{l}\text { Water-level } \\
\text { elevation }(\mathrm{m})\end{array}$ \\
\hline 649 & $01-02-89$ & 7.99 & 242.44 \\
\hline 649 & $01-11-89$ & 7.95 & 242.48 \\
\hline 649 & $01-17-89$ & 7.74 & 242.69 \\
\hline 649 & $01-27-89$ & 7.99 & 242.44 \\
\hline 649 & $01-31-89$ & 8.02 & 242.40 \\
\hline 649 & $02-10-89$ & 7.90 & 242.52 \\
\hline 649 & $02-17-89$ & 7.88 & 242.55 \\
\hline 649 & $02-24-89$ & 7.98 & 242.45 \\
\hline 649 & $03-03-89$ & 8.08 & 242.35 \\
\hline 649 & $03-15-89$ & 8.03 & 242.40 \\
\hline 649 & $03-22-89$ & 7.89 & 242.54 \\
\hline 649 & $03-28-89$ & 8.05 & 242.37 \\
\hline 649 & 04-07-89 & 8.00 & 242.42 \\
\hline 649 & $05-04-89$ & 8.70 & 242.27 \\
\hline 649 & $05-12-89$ & 8.69 & 242.28 \\
\hline 649 & $05-17-89$ & 8.61 & 242.35 \\
\hline 649 & $05-26-89$ & 8.64 & 242.33 \\
\hline 649 & $06-01-89$ & 8.61 & 242.35 \\
\hline 649 & $06-09-89$ & 8.58 & 242.39 \\
\hline 649 & $06-16-89$ & 8.52 & 242.45 \\
\hline 649 & $06-23-89$ & 8.35 & 242.62 \\
\hline 649 & $06-28-89$ & 8.47 & 242.49 \\
\hline 649 & $07-07-89$ & 8.48 & 242.48 \\
\hline 649 & $07-14-89$ & 8.61 & 242.35 \\
\hline 649 & $07-21-89$ & 8.63 & 242.34 \\
\hline 649 & $07-28-89$ & 8.76 & 242.21 \\
\hline 649 & $08-04-89$ & 8.93 & 242.04 \\
\hline 649 & $08-11-89$ & 8.87 & 242.10 \\
\hline 649 & $08-18-89$ & 8.84 & 242.13 \\
\hline 649 & $08-30-89$ & 8.69 & 242.28 \\
\hline 649 & $09-07-89$ & 8.70 & 242.27 \\
\hline 649 & $09-18-89$ & 8.69 & 242.28 \\
\hline 649 & $09-27-89$ & 8.72 & 242.24 \\
\hline $650 \mathrm{~A}$ & $10-04-88$ & Dry & \\
\hline $650 \mathrm{~A}$ & $10-24-88$ & Dry & \\
\hline $650 \mathrm{~A}$ & $11-01-88$ & Dry & \\
\hline $650 \mathrm{~A}$ & $11-07-88$ & Dry & \\
\hline $650 \mathrm{~A}$ & $11-14-88$ & Dry & \\
\hline $650 \mathrm{~A}$ & $11-21-88$ & Dry & \\
\hline $650 \mathrm{~A}$ & $11-28-88$ & Dry & \\
\hline $650 \mathrm{~A}$ & $12-06-88$ & Dry & \\
\hline $650 \mathrm{~A}$ & $12-12-88$ & Dry & \\
\hline $650 \mathrm{~A}$ & $12-20-88$ & Dry & \\
\hline $650 \mathrm{~A}$ & $12-27-88$ & Dry & \\
\hline $650 \mathrm{~A}$ & $01-02-89$ & Dry & \\
\hline $650 \mathrm{~A}$ & $01-11.89$ & Dry & \\
\hline $650 \mathrm{~A}$ & $01-17-89$ & Dry & \\
\hline $650 \mathrm{~A}$ & $01-27-89$ & Dry & \\
\hline $650 \mathrm{~A}$ & $01-31-89$ & Dry & \\
\hline $650 \mathrm{~A}$ & $02-10-89$ & Dry & \\
\hline $650 \mathrm{~A}$ & $02-17-89$ & Dry & \\
\hline $650 \mathrm{~A}$ & $02-24-89$ & Dry & \\
\hline $650 \mathrm{~A}$ & $03-03-89$ & Dry & \\
\hline $650 \mathrm{~A}$ & $03-15-89$ & Dry & \\
\hline $650 \mathrm{~A}$ & $03-22-89$ & Dry & \\
\hline $650 \mathrm{~A}$ & $03-28-89$ & Dry & \\
\hline $650 \mathrm{~A}$ & $04-07-89$ & Dry & \\
\hline $650 \mathrm{~A}$ & $04-14-89$ & Dry & \\
\hline
\end{tabular}


Table A.1 (continued)

\begin{tabular}{|c|c|c|c|}
\hline $\begin{array}{l}\text { Well } \\
\text { No. }\end{array}$ & $\begin{array}{c}\text { Date } \\
\text { measured }\end{array}$ & $\begin{array}{l}\text { Depth to } \\
\text { water (m) }\end{array}$ & $\begin{array}{l}\text { Water-level } \\
\text { elevation }(m)\end{array}$ \\
\hline $650 \mathrm{~A}$ & $04-22-89$ & Dry & \\
\hline $650 \mathrm{~A}$ & $05-04-89$ & Dry & \\
\hline $650 \mathrm{~A}$ & $05-12-89$ & Dry & \\
\hline $650 \mathrm{~A}$ & $05-17-89$ & Dry & \\
\hline $650 \mathrm{~A}$ & $05-26-89$ & Dry & \\
\hline $650 \mathrm{~A}$ & $06-01-89$ & Dry & \\
\hline $650 \mathrm{~A}$ & $06-09-89$ & Dry & \\
\hline $650 \mathrm{~A}$ & $06-16-89$ & Dry & \\
\hline $650 \mathrm{~A}$ & $06-23-89$ & Dry & \\
\hline $650 \mathrm{~A}$ & $06-28-89$ & Dry & \\
\hline $650 \mathrm{~A}$ & $07-07-89$ & Dry & \\
\hline $650 \mathrm{~A}$ & $07-14-89$ & Dry & \\
\hline $650 \mathrm{~A}$ & $07-21-89$ & Dry & \\
\hline $650 \mathrm{~A}$ & $07-28-89$ & Dry & \\
\hline $650 \mathrm{~A}$ & $08-04-89$ & Dry & \\
\hline $650 \mathrm{~A}$ & $08-11-89$ & Dry & \\
\hline $650 \mathrm{~A}$ & $08-18-89$ & Dry & \\
\hline $650 \mathrm{~A}$ & $08-30-89$ & Dry & \\
\hline $650 \mathrm{~A}$ & $09-07-89$ & Dry & \\
\hline $650 \mathrm{~A}$ & $09-18-89$ & Dry & \\
\hline $650 \mathrm{~A}$ & $09-27-89$ & Dry & \\
\hline $650 \mathrm{~A}$ & $01-15-90$ & Dry & \\
\hline $650 \mathrm{~A}$ & $02-15-90$ & Dry & \\
\hline $650 \mathrm{~A}$ & $03-14-90$ & Dry & \\
\hline $650 \mathrm{~A}$ & $04-23-90$ & Dry & \\
\hline $650 \mathrm{~A}$ & $05-21-90$ & Dry & \\
\hline $650 \mathrm{~A}$ & $06-26-90$ & Dry & \\
\hline $650 \mathrm{~A}$ & $07-25-90$ & Dry & \\
\hline $650 \mathrm{~A}$ & $08-23-90$ & Dry & \\
\hline $650 \mathrm{~A}$ & $09-25-90$ & Dry & \\
\hline $650 \mathrm{~B}$ & $01-12-88$ & Dry & \\
\hline $650 \mathrm{~B}$ & $02-04-88$ & 12.18 & 237.86 \\
\hline $650 \mathrm{~B}$ & $03-03-88$ & 12.06 & 237.98 \\
\hline $650 \mathrm{~B}$ & $04-08-88$ & 11.96 & 238.08 \\
\hline $650 \mathrm{~B}$ & $05-05-88$ & 12.02 & 238.02 \\
\hline $650 \mathrm{~B}$ & $06-09-88$ & 12.16 & 237.88 \\
\hline $650 \mathrm{~B}$ & $06-29-88$ & 12.22 & 237.82 \\
\hline $650 \mathrm{~B}$ & $11-14-88$ & 12.20 & 237.84 \\
\hline $650 \mathrm{~B}$ & $11-21-88$ & 12.13 & 237.91 \\
\hline $650 \mathrm{~B}$ & $11-28-88$ & 12.07 & 237.97 \\
\hline $650 \mathrm{~B}$ & $12-06-88$ & 12.16 & 237.88 \\
\hline $650 \mathrm{~B}$ & $12-12-88$ & 12.41 & 237.63 \\
\hline $650 \mathrm{~B}$ & $12-20-88$ & 12.09 & 237.95 \\
\hline $650 \mathrm{~B}$ & $12-27-88$ & 11.95 & 238.09 \\
\hline $650 \mathrm{~B}$ & $01-02-89$ & 11.78 & 238.26 \\
\hline $650 \mathrm{~B}$ & $01-11-89$ & 12.04 & 238.00 \\
\hline $650 \mathrm{~B}$ & $01-17-89$ & 11.59 & 238.45 \\
\hline $650 \mathrm{~B}$ & $01-27-89$ & 11.66 & 238.38 \\
\hline $650 \mathrm{~B}$ & $01-31-89$ & 11.86 & 238.18 \\
\hline $650 \mathrm{~B}$ & $02-10-89$ & 11.69 & 238.35 \\
\hline $650 \mathrm{~B}$ & $02-17-89$ & 11.63 & 238.41 \\
\hline $650 \mathrm{~B}$ & $02-24-89$ & 11.95 & 238.09 \\
\hline $650 \mathrm{~B}$ & $03-03-89$ & 11.51 & 238.53 \\
\hline $650 \mathrm{~B}$ & $03-15-89$ & 10.09 & 239.95 \\
\hline $650 \mathrm{~B}$ & $03-22-89$ & 11.78 & 238.26 \\
\hline $650 \mathrm{~B}$ & $03-28-89$ & 11.59 & 238.45 \\
\hline $650 \mathrm{~B}$ & $04-07-89$ & 11.51 & 238.53 \\
\hline $650 \mathrm{~B}$ & $04-14-89$ & 11.61 & 238.43 \\
\hline
\end{tabular}


Table A.1 (continued)

\begin{tabular}{|c|c|c|c|}
\hline $\begin{array}{l}\text { Well } \\
\text { No. }\end{array}$ & $\begin{array}{c}\text { Date } \\
\text { measured }\end{array}$ & $\begin{array}{l}\text { Depth to } \\
\text { water }(m)\end{array}$ & $\begin{array}{l}\text { Water-level } \\
\text { elevation }(\mathrm{m})\end{array}$ \\
\hline $650 \mathrm{~B}$ & $04-22-89$ & 11.79 & 238.24 \\
\hline $650 \mathrm{~B}$ & $05-04-89$ & 11.81 & 238.22 \\
\hline $650 \mathrm{~B}$ & $05-12-89$ & 11.99 & 238.05 \\
\hline $650 \mathrm{~B}$ & $05-26-89$ & 11.59 & 238.45 \\
\hline $650 \mathrm{~B}$ & $06-01-89$ & 11.99 & 238.04 \\
\hline $650 \mathrm{~B}$ & $06-09-89$ & 11.81 & 238.22 \\
\hline $650 \mathrm{~B}$ & $06-16-89$ & 11.76 & 238.27 \\
\hline $650 \mathrm{~B}$ & $06-23-89$ & 11.65 & 238.39 \\
\hline $650 \mathrm{~B}$ & $06-28-89$ & 11.65 & 238.39 \\
\hline $650 \mathrm{~B}$ & $07-07-89$ & 11.64 & 238.40 \\
\hline $650 \mathrm{~B}$ & $07-14-89$ & 11.97 & 238.07 \\
\hline $650 \mathrm{~B}$ & $07-21-89$ & 11.95 & 238.09 \\
\hline $650 \mathrm{~B}$ & $07-28-89$ & 12.09 & 237.94 \\
\hline $650 \mathrm{~B}$ & $08-04-89$ & 11.97 & 238.07 \\
\hline $650 \mathrm{~B}$ & $08-11-89$ & 11.91 & 238.12 \\
\hline $650 \mathrm{~B}$ & $08-18-89$ & 11.89 & 238.15 \\
\hline $650 \mathrm{~B}$ & $08-30-89$ & 11.94 & 238.09 \\
\hline $650 \mathrm{~B}$ & $09.07-89$ & 11.91 & 238.12 \\
\hline $650 \mathrm{~B}$ & $09-18-89$ & 11.95 & 238.09 \\
\hline $650 \mathrm{~B}$ & $09-27-89$ & 11.97 & 238.07 \\
\hline $650 \mathrm{~B}$ & $01-15-90$ & Blocked & \\
\hline $650 \mathrm{~B}$ & $02-15-90$ & Blocked & \\
\hline $650 \mathrm{~B}$ & $03-15-90$ & Blocked & \\
\hline $650 \mathrm{~B}$ & $04-23-90$ & Blocked & \\
\hline $650 \mathrm{~B}$ & $05-21-90$ & Blocked & \\
\hline $650 \mathrm{~B}$ & $06-26-90$ & Blocked & \\
\hline $650 \mathrm{~B}$ & $07-25-90$ & Blocked & \\
\hline $650 \mathrm{~B}$ & $08-23-90$ & Blocked & \\
\hline $650 \mathrm{~B}$ & $09-25-90$ & Blocked & \\
\hline 654 & $01-15-88$ & 0.90 & 235.41 \\
\hline 654 & $02-04-88$ & 0.70 & 235.60 \\
\hline 654 & $03-03-88$ & 0.72 & 235.58 \\
\hline 654 & $04-08-88$ & 0.71 & 235.59 \\
\hline 654 & $05-05-88$ & 0.88 & 235.43 \\
\hline 654 & $06-09-88$ & 1.22 & 235.08 \\
\hline 654 & $06-29-88$ & 1.31 & 234.99 \\
\hline 654 & $07-28-88$ & 1.02 & 235.28 \\
\hline 654 & $08-31-88$ & 1.35 & 234.95 \\
\hline 654 & $10-05-88$ & 1.23 & 235.07 \\
\hline 654 & $10-24-88$ & 1.27 & 235.03 \\
\hline 654 & $11-01-88$ & 1.21 & 235.09 \\
\hline 654 & $11-01-88$ & 1.28 & 235.02 \\
\hline 654 & $11-07-88$ & 1.16 & 235.14 \\
\hline 654 & $11-14-88$ & 1.03 & 235.27 \\
\hline 654 & $11-21-88$ & 0.90 & 235.41 \\
\hline 654 & $11-28-88$ & 0.83 & 235.48 \\
\hline 654 & $12-05-88$ & 0.80 & 235.50 \\
\hline 654 & $12-06-88$ & 0.83 & 235.48 \\
\hline 654 & $12-12-88$ & 0.86 & 235.44 \\
\hline 654 & $12-20-88$ & 0.92 & 235.38 \\
\hline 654 & $12-27-88$ & 0.77 & 235.53 \\
\hline 654 & $01-02-89$ & 0.63 & 235.68 \\
\hline 654 & $01-06-89$ & 0.54 & 235.76 \\
\hline 654 & $01-11-89$ & 0.53 & 235.77 \\
\hline 654 & $01-17-89$ & 0.36 & 235.95 \\
\hline 654 & $01-27-89$ & 0.52 & 235.78 \\
\hline 654 & $01-31-89$ & 0.61 & 235.69 \\
\hline 654 & $02-08-89$ & 0.52 & 235.78 \\
\hline
\end{tabular}


Table A.1 (continued)

\begin{tabular}{|c|c|c|c|}
\hline $\begin{array}{l}\text { Well } \\
\text { No. }\end{array}$ & $\begin{array}{c}\text { Date } \\
\text { measured }\end{array}$ & $\begin{array}{l}\text { Depth to } \\
\text { water }(\mathrm{m})\end{array}$ & $\begin{array}{l}\text { Water-level } \\
\text { elevation }(\mathrm{m})\end{array}$ \\
\hline 654 & $02-10-89$ & 0.51 & 235.79 \\
\hline 654 & $02-17-89$ & 0.48 & 235.82 \\
\hline 654 & $02-24-39$ & 0.46 & 235.84 \\
\hline 654 & $03-03-89$ & 0.34 & 235.96 \\
\hline 654 & $03-09-89$ & 0.30 & 236.00 \\
\hline 654 & $03-15-89$ & 0.36 & 235.94 \\
\hline 654 & $03-22-89$ & 0.39 & 235.91 \\
\hline 654 & $03-28-89$ & 0.39 & 235.91 \\
\hline 654 & $04-07-89$ & 0.36 & 235.95 \\
\hline 654 & $04-14-89$ & 0.41 & 235.90 \\
\hline 654 & $04-22-89$ & 0.50 & 235.80 \\
\hline 654 & $05-04-89$ & 0.59 & 235.72 \\
\hline 654 & $05-12-89$ & 0.45 & 235.86 \\
\hline 654 & $05-17-89$ & 0.51 & 235.79 \\
\hline 654 & $05-26-89$ & 0.53 & 235.77 \\
\hline 654 & $06-01-89$ & 0.61 & 235.69 \\
\hline 654 & $06-09-89$ & 0.50 & 235.80 \\
\hline 654 & $06-16-89$ & 0.46 & 235.84 \\
\hline 654 & $06-23-89$ & 0.23 & 236.07 \\
\hline 654 & $06-28-89$ & 0.28 & 236.02 \\
\hline 654 & $07-07-89$ & 0.27 & 236.03 \\
\hline 654 & $07-14-89$ & 0.33 & 235.97 \\
\hline 654 & $07-21-89$ & 0.30 & 236.00 \\
\hline 654 & $07-28-89$ & 0.53 & 235.77 \\
\hline 654 & $08-04-89$ & 0.57 & 235.73 \\
\hline 654 & $08-11-89$ & 0.55 & 235.76 \\
\hline 654 & $08-18-89$ & 0.50 & 235.80 \\
\hline 654 & $08-30-89$ & 0.56 & 235.74 \\
\hline 654 & $09-07-89$ & 0.49 & 235.81 \\
\hline 654 & $09-18-89$ & 0.47 & 235.84 \\
\hline 654 & $09-27-89$ & 0.52 & 235.78 \\
\hline 654 & $01-15-90$ & 0.29 & 236.01 \\
\hline 654 & $02-15-90$ & 0.00 & 236.30 \\
\hline 654 & $03-14-90$ & 0.00 & 236.30 \\
\hline 654 & $04-23-90$ & 0.15 & 236.15 \\
\hline 654 & $05-21-90$ & 0.09 & 236.21 \\
\hline 654 & $06-26-90$ & 0.61 & 235.69 \\
\hline 654 & $07-25-90$ & 0.50 & 235.80 \\
\hline 654 & $08-23-90$ & 0.59 & 235.72 \\
\hline 654 & $09-25-90$ & 0.83 & 235.48 \\
\hline 655 & $01-12-88$ & 13.26 & 242.73 \\
\hline 655 & $02-04-88$ & 12.80 & 243.19 \\
\hline 655 & $03-03-88$ & 12.62 & 243.37 \\
\hline 655 & $04-08-88$ & 12.45 & 243.54 \\
\hline 655 & $05-0=-88$ & 12.52 & 243.47 \\
\hline 655 & $06-09-88$ & 13.00 & 242.99 \\
\hline 655 & $06-29-88$ & 13.20 & 242.79 \\
\hline 655 & $07-28-88$ & 13.09 & 242.89 \\
\hline 655 & $08-31-88$ & 13.30 & 242.69 \\
\hline 655 & $10-04-88$ & 13.24 & 242.75 \\
\hline 655 & $10-05-88$ & 13.30 & 242.69 \\
\hline 655 & $10-24-88$ & 13.32 & 242.67 \\
\hline 655 & $11-01-88$ & 13.27 & 242.72 \\
\hline 655 & $11-01-88$ & 13.39 & 242.60 \\
\hline 655 & $11-07-88$ & 13.35 & 242.64 \\
\hline 655 & $11-14-88$ & 13.23 & 242.76 \\
\hline 655 & $11-21-88$ & 13.05 & 242.94 \\
\hline 655 & $11-28-88$ & 12.91 & 243.08 \\
\hline
\end{tabular}


Table A.1 (continued)

\begin{tabular}{|c|c|c|c|}
\hline $\begin{array}{l}\text { Well } \\
\text { No. }\end{array}$ & $\begin{array}{c}\text { Date } \\
\text { measured }\end{array}$ & $\begin{array}{l}\text { Depth to } \\
\text { water }(m)\end{array}$ & $\begin{array}{l}\text { Water-level } \\
\text { elevation }(\mathrm{m})\end{array}$ \\
\hline 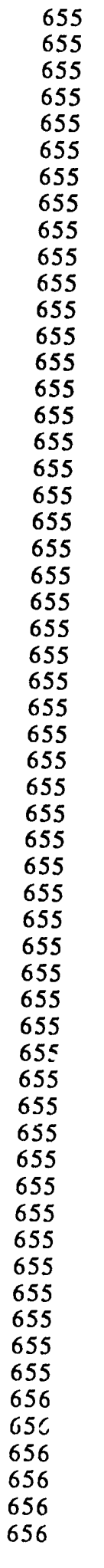 & $\begin{array}{l}12-05-88 \\
12-06-88 \\
12-12-88 \\
12-20-88 \\
12-27-88 \\
01-02-89 \\
01-06-89 \\
01-11-89 \\
01-17-89 \\
01-27-89 \\
01-31-89 \\
02-08-89 \\
02-10-89 \\
02-17-89 \\
02-24-89 \\
03-03-89 \\
03-09-89 \\
03-15-89 \\
03-22-89 \\
03-28-89 \\
04-07-89 \\
04-14-89 \\
04-22-89 \\
05-04-89 \\
05-12-89 \\
05-17-89 \\
05-26-89 \\
06-01-89 \\
06-09-89 \\
06-16-89 \\
06-23-89 \\
06-28-89 \\
07-07-89 \\
07-14-89 \\
07-21-89 \\
07-28-89 \\
08-04-89 \\
08-11-89 \\
08-18-89 \\
08-30-89 \\
09-07-89 \\
09-18-89 \\
09-27-89 \\
01-15-90 \\
02-15-90 \\
03-14-90 \\
04-23-90 \\
05-21-90 \\
06-26-90 \\
07-25-90 \\
08-23-90 \\
09-25-90 \\
01-15-88 \\
02-04-88 \\
04-03-88 \\
05-05-88 \\
06-09-88\end{array}$ & $\begin{array}{l}12.90 \\
12.80 \\
12.83 \\
12.88 \\
12.72 \\
12.50 \\
12.37 \\
12.25 \\
11.75 \\
11.98 \\
12.08 \\
12.15 \\
12.14 \\
12.04 \\
12.61 \\
11.75 \\
11.61 \\
11.60 \\
11.93 \\
11.93 \\
11.89 \\
11.84 \\
12.20 \\
12.40 \\
12.20 \\
12.31 \\
12.37 \\
12.40 \\
12.37 \\
12.35 \\
11.74 \\
11.70 \\
11.87 \\
12.05 \\
12.05 \\
12.23 \\
12.41 \\
12.40 \\
12.41 \\
12.64 \\
12.62 \\
12.50 \\
12.61 \\
12.05 \\
11.58 \\
11.90 \\
12.32 \\
12.06 \\
12.50 \\
12.42 \\
12.53 \\
12.87 \\
12.95 \\
12.37 \\
11.84 \\
11.71 \\
11.52 \\
12.18 \\
\end{array}$ & $\begin{array}{l}243.09 \\
243.18 \\
243.16 \\
243.11 \\
243.27 \\
243.49 \\
243.62 \\
243.74 \\
244.24 \\
244.01 \\
243.91 \\
243.84 \\
243.84 \\
243.95 \\
243.38 \\
244.24 \\
244.38 \\
244.39 \\
244.06 \\
244.06 \\
244.10 \\
244.15 \\
243.79 \\
243.59 \\
243.79 \\
243.68 \\
243.62 \\
243.59 \\
243.62 \\
243.64 \\
244.25 \\
244.29 \\
244.12 \\
243.93 \\
243.94 \\
243.75 \\
243.58 \\
243.59 \\
243.58 \\
243.35 \\
243.37 \\
243.49 \\
243.38 \\
243.93 \\
244.41 \\
244.09 \\
243.67 \\
243.92 \\
243.49 \\
243.57 \\
243.46 \\
243.12 \\
245.52 \\
246.10 \\
246.63 \\
246.77 \\
246.95 \\
246.29\end{array}$ \\
\hline
\end{tabular}


Table A.1 (continued)

\begin{tabular}{|c|c|c|c|}
\hline $\begin{array}{l}\text { Well } \\
\text { No. }\end{array}$ & $\begin{array}{c}\text { Date } \\
\text { measured }\end{array}$ & $\begin{array}{l}\text { Depth to } \\
\text { water }(m)\end{array}$ & $\begin{array}{l}\text { Water-level } \\
\text { elevation (m) }\end{array}$ \\
\hline 656 & $06-29-88$ & 12.48 & 245.99 \\
\hline 656 & $07-28-88$ & 12.64 & 245.83 \\
\hline 656 & $08-31-88$ & 12.80 & 245.67 \\
\hline 656 & $10-04-88$ & 12.60 & 245.87 \\
\hline 656 & $10-04-88$ & 12.89 & 245.59 \\
\hline 656 & $10-24-88$ & 12.95 & 245.53 \\
\hline 656 & $11-01-88$ & 12.84 & 245.64 \\
\hline 656 & $11-01-88$ & 13.02 & 245.45 \\
\hline 656 & $11-07-88$ & 13.03 & 245.44 \\
\hline 656 & $11-14-88$ & 12.93 & 245.54 \\
\hline 656 & $11-28-88$ & 12.56 & 245.91 \\
\hline 656 & $12-05-88$ & 12.39 & 246.09 \\
\hline 656 & $12-06-88$ & 12.35 & 246.12 \\
\hline 656 & $12-12-88$ & 12.26 & 246.21 \\
\hline 656 & $12-20-88$ & 12.27 & 246.20 \\
\hline 656 & $12-27-88$ & 12.25 & 246.22 \\
\hline 656 & $01-02-89$ & 12.09 & 246.38 \\
\hline 656 & $01-05-89$ & 11.87 & 246.60 \\
\hline 656 & $01-11-89$ & 10.70 & 247.78 \\
\hline 656 & $01-17-89$ & 11.28 & 247.19 \\
\hline 656 & $01-27-89$ & 10.82 & 247.65 \\
\hline 656 & $01-31-89$ & 10.92 & 247.55 \\
\hline 656 & $02-08-89$ & 11.14 & 247.33 \\
\hline 656 & $02-10-89$ & 11.30 & 247.17 \\
\hline 656 & $02-12-89$ & 11.36 & 247.12 \\
\hline 656 & $02-24-89$ & 11.63 & 246.85 \\
\hline 656 & $03-03-89$ & 11.59 & 246.89 \\
\hline 656 & $03-09-89$ & 10.61 & 247.87 \\
\hline 656 & $03-15-89$ & 10.37 & 248.11 \\
\hline 656 & $03-22-89$ & 10.73 & 247.74 \\
\hline 656 & $03-28-89$ & 12.22 & 246.25 \\
\hline 656 & $04-07-89$ & 12.15 & 246.32 \\
\hline 656 & $04-14-89$ & 12.19 & 246.29 \\
\hline 656 & $04-22-89$ & 12.25 & 2.46 .23 \\
\hline 656 & $05-04-89$ & 12.55 & 245.92 \\
\hline 656 & $05-12-89$ & 12.70 & 245.77 \\
\hline 656 & $05-17-89$ & 12.65 & 245.82 \\
\hline 656 & $05-26-89$ & 11.14 & 247.33 \\
\hline 656 & $06-01-89$ & 11.23 & 247.24 \\
\hline 656 & $06-09-89$ & 11.34 & 247.13 \\
\hline 656 & $06-16-89$ & 11.28 & 247.19 \\
\hline 656 & $06-23-89$ & 11.03 & 247.4 .5 \\
\hline 656 & $06-28-89$ & 10.70 & 247.78 \\
\hline 656 & $07-07-89$ & 10.75 & 247.73 \\
\hline 656 & $07-14-89$ & 12.32 & 246.15 \\
\hline 656 & $07-21-89$ & 11.99 & 246.48 \\
\hline 656 & $07-28-89$ & 11.30 & 247.17 \\
\hline 656 & $08-04-89$ & 11.38 & 247.09 \\
\hline 656 & $08-11-89$ & 11.38 & 247.09 \\
\hline 656 & $08-18-89$ & 11.40 & 247.07 \\
\hline 656 & $08-30-89$ & 11.91 & 246.56 \\
\hline 656 & $09-07-89$ & 11.99 & 246.48 \\
\hline 656 & $09-18-89$ & 11.89 & 246.58 \\
\hline 656 & $09-27-89$ & 11.97 & 246.51 \\
\hline 656 & $01-15-90$ & 11.15 & 247.32 \\
\hline 656 & $02-15-90$ & 10.33 & 248.14 \\
\hline 656 & $03-14-90$ & 10.58 & 247.89 \\
\hline 656 & $04-23-90$ & 11.10 & 247.38 \\
\hline
\end{tabular}


Table A.1 (continued)

\begin{tabular}{lccc}
\hline $\begin{array}{l}\text { Well } \\
\text { No. }\end{array}$ & $\begin{array}{c}\text { Date } \\
\text { measured }\end{array}$ & $\begin{array}{c}\text { Depth to } \\
\text { water }(\mathrm{m})\end{array}$ & $\begin{array}{c}\text { Water-level } \\
\text { elevation }(\mathrm{m})\end{array}$ \\
\hline 656 & $05-21-90$ & 10.82 & 247.65 \\
656 & $06-26-90$ & 11.54 & 246.93 \\
656 & $07-25-90$ & 11.74 & 246.73 \\
656 & $08-23-90$ & 11.83 & 246.64 \\
656 & $09-25-90$ & 12.23 & 246.24 \\
\hline
\end{tabular}




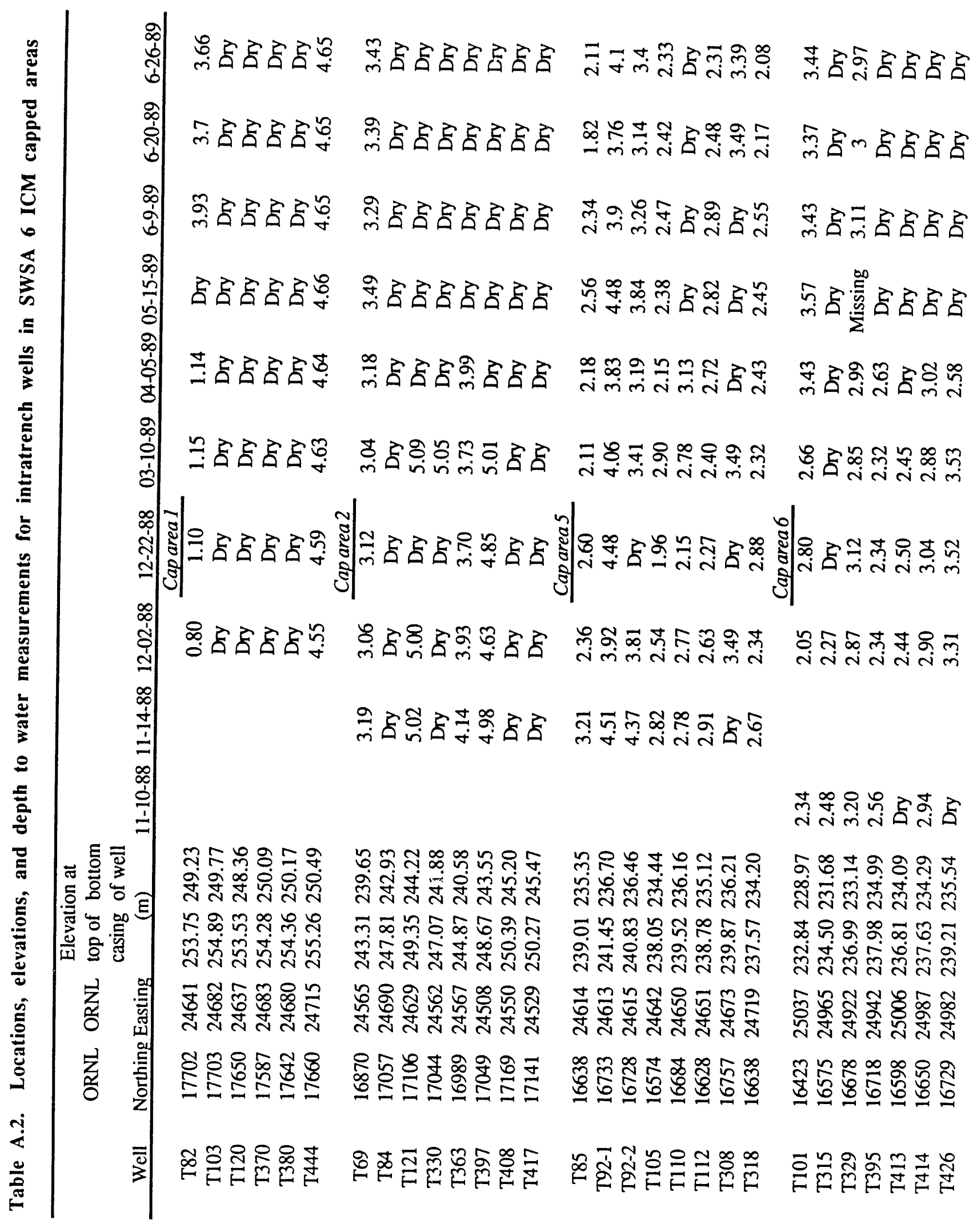




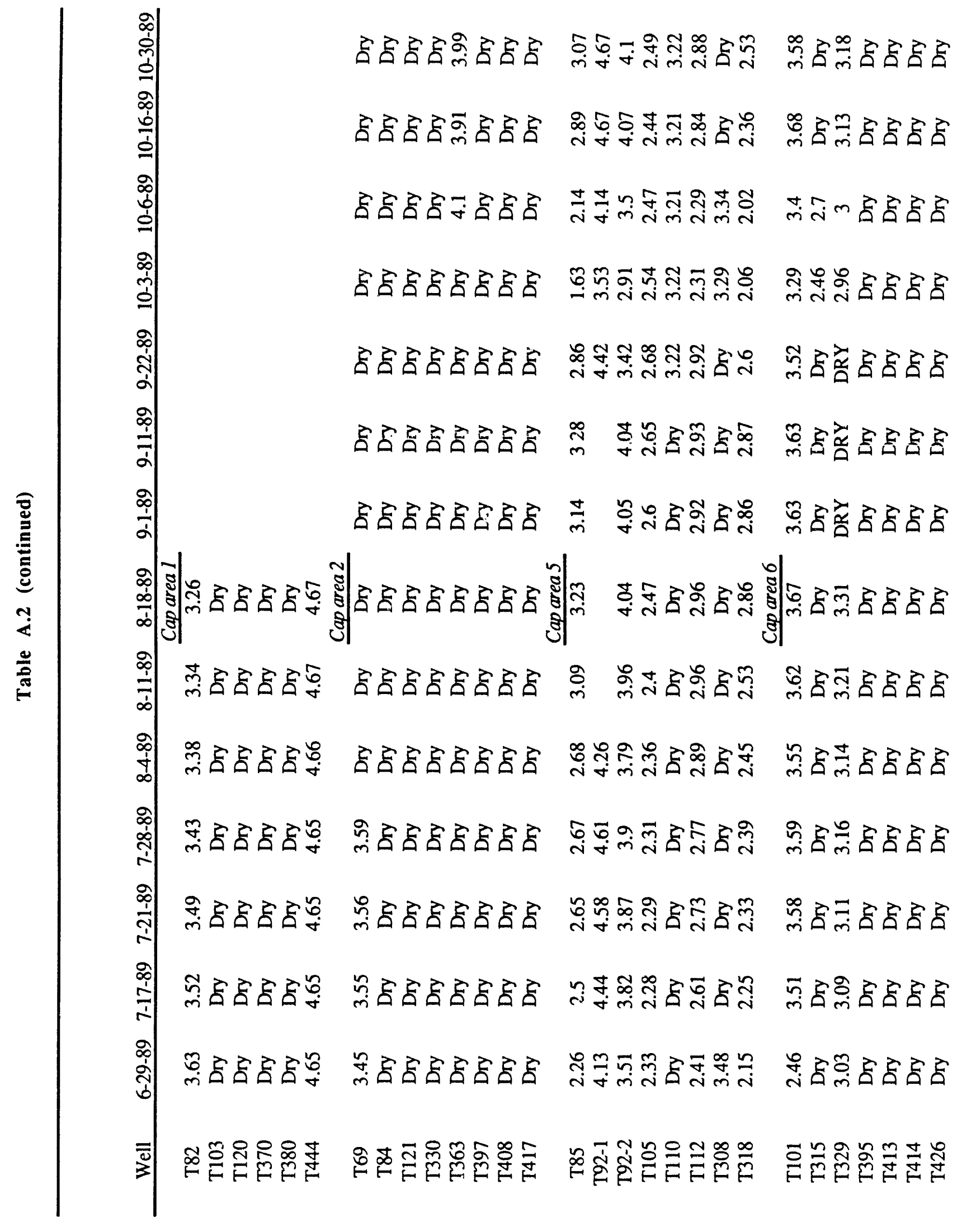




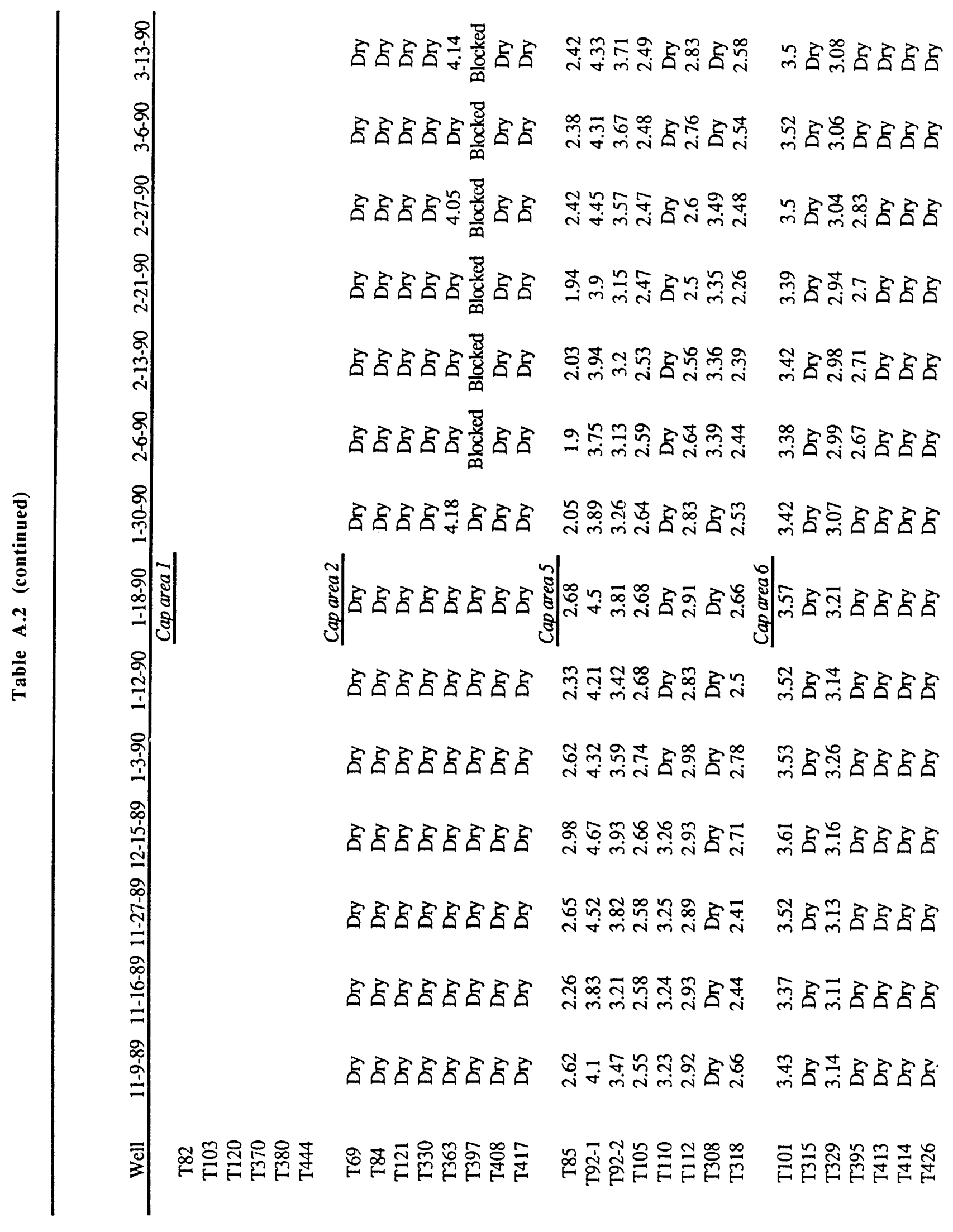




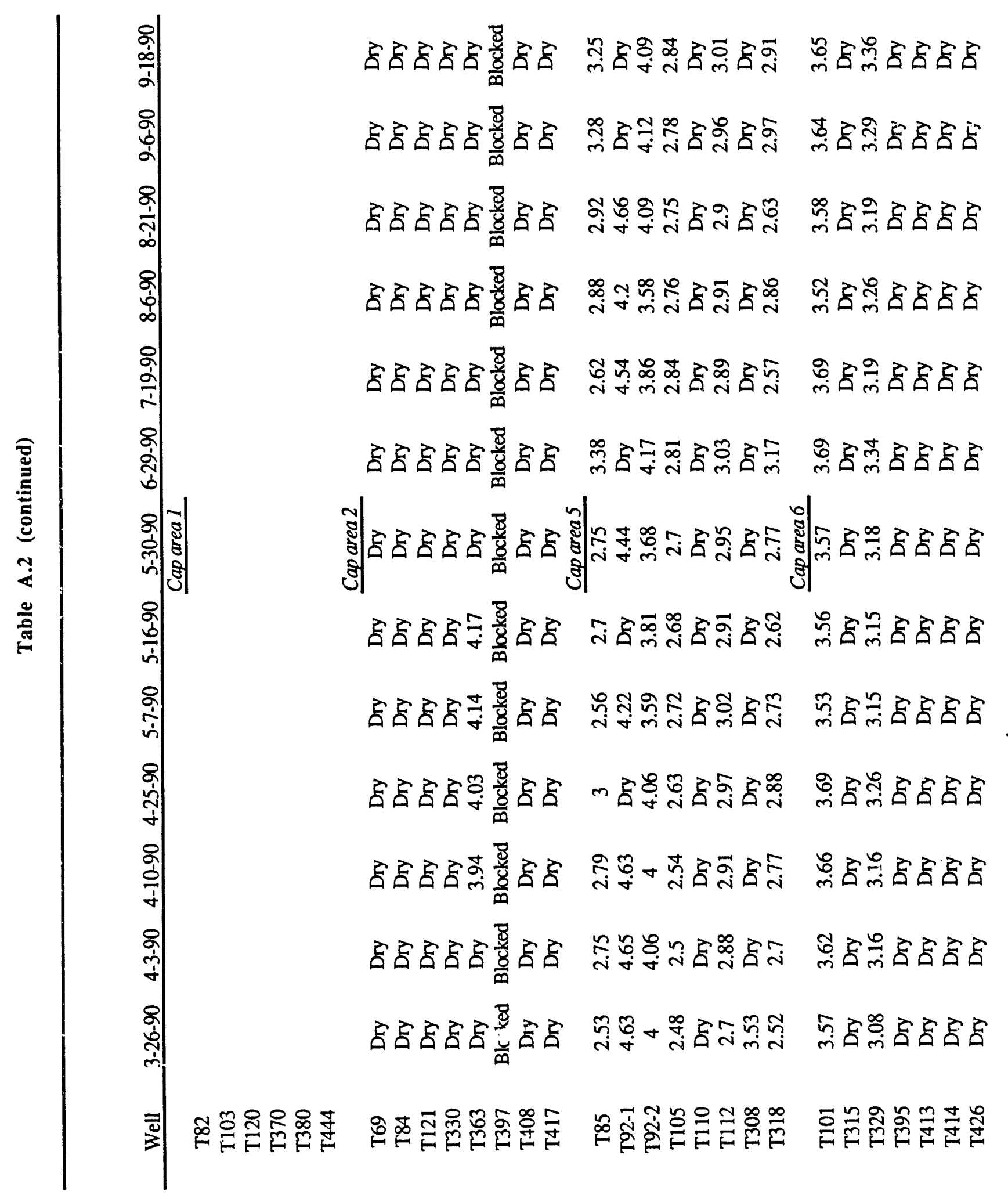




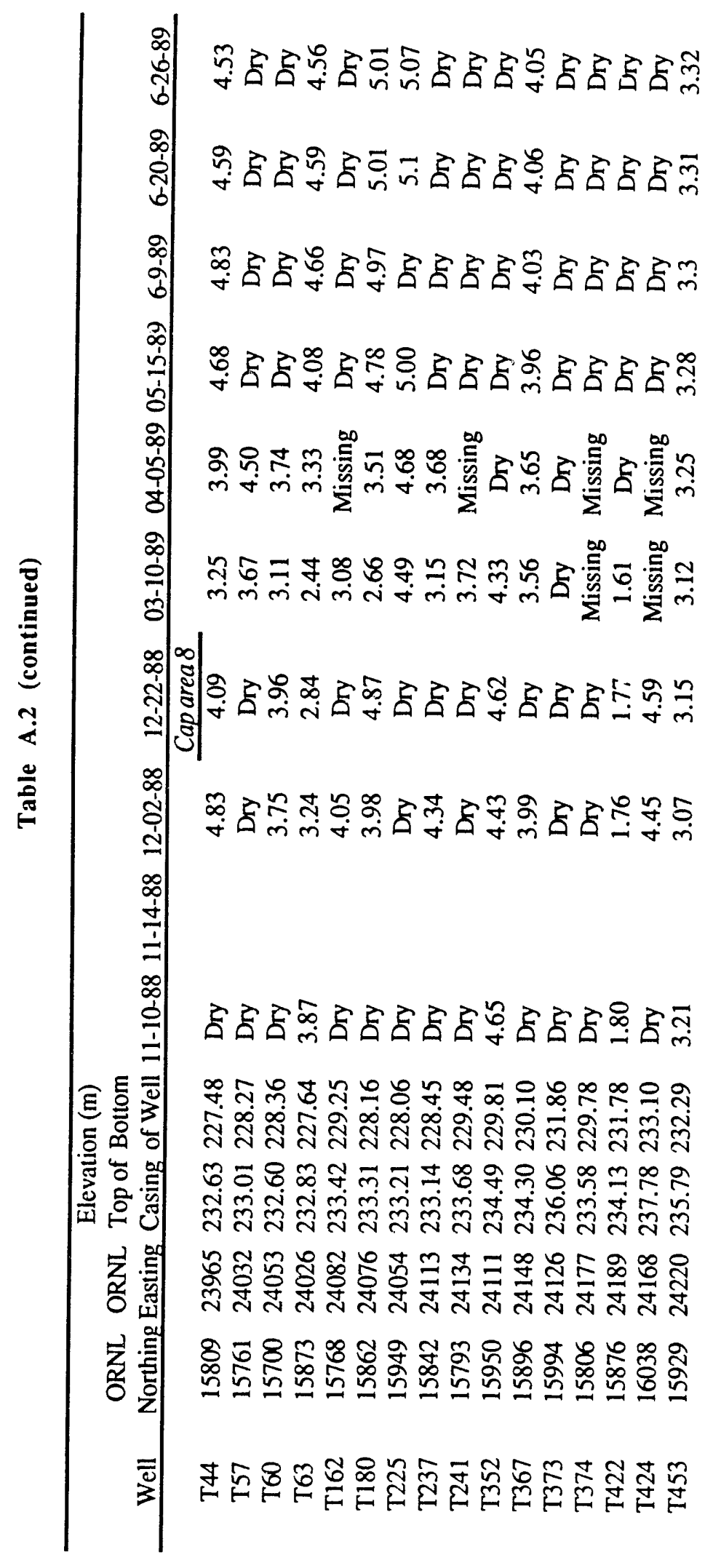




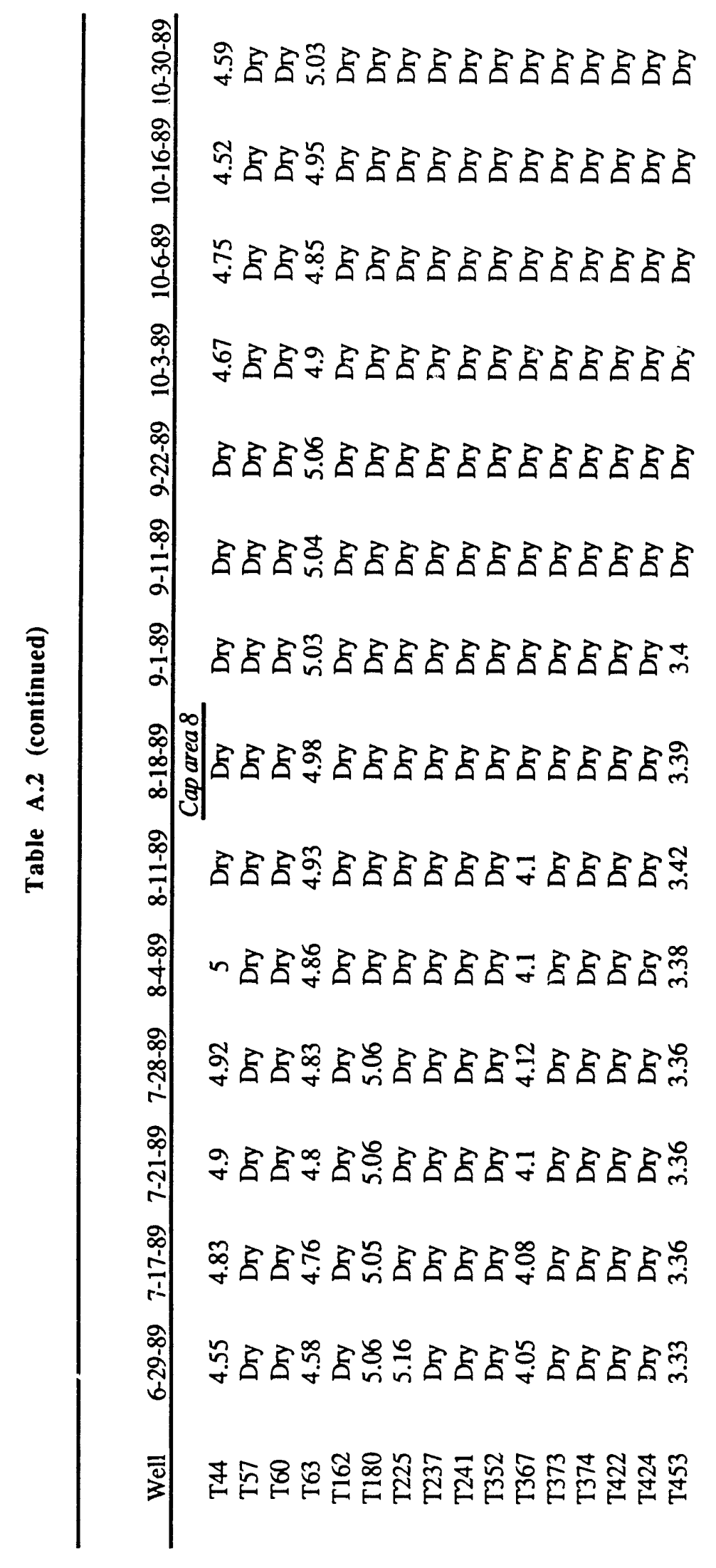




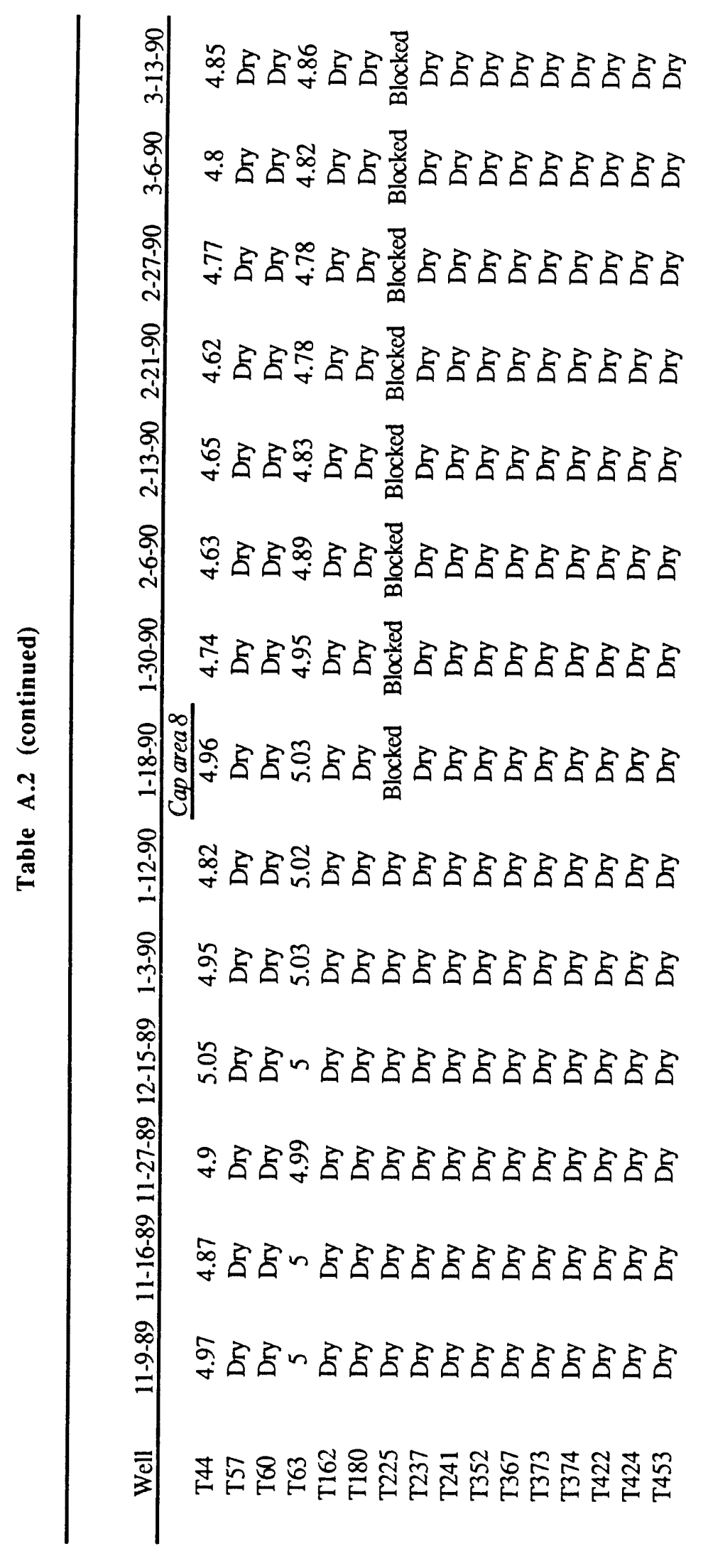




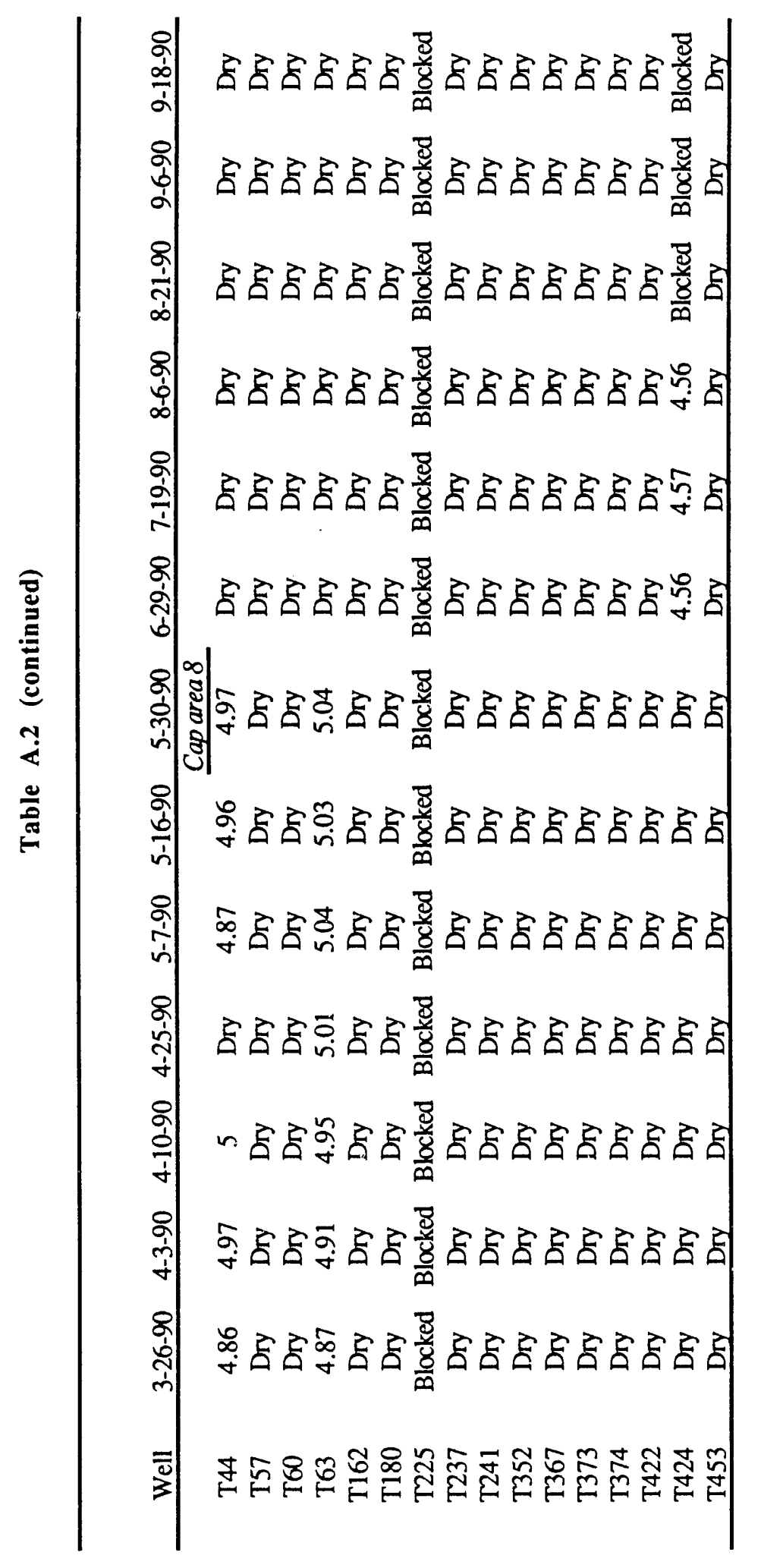


Table A.3. Daily rainfall in SWSA 6 from October 1988 through September 1990

\begin{tabular}{|c|c|c|c|c|c|c|c|}
\hline Date & $\begin{array}{l}\text { Precipitation } \\
\text { (mm) }\end{array}$ & Date & $\begin{array}{l}\text { Precipitation } \\
\text { (mm) }\end{array}$ & Date & $\begin{array}{l}\text { Precipitation } \\
\text { (mm) }\end{array}$ & Date & $\begin{array}{l}\text { Precipitation } \\
(\mathrm{mm})\end{array}$ \\
\hline $10 / 01 / 88$ & 12.95 & $11 / 19 / 88$ & 21.08 & $01 / 07 / 89$ & 0.00 & $02 / 25 / 89$ & 0.00 \\
\hline $10 / 02 / 88$ & 4.83 & $11 / 20 / 88$ & 20.57 & $01 / 08 / 89$ & 26.61 & $02 / 26 / 89$ & 0.00 \\
\hline $10 / 03 / 88$ & 0.00 & $11 / 21 / 88$ & 0.00 & $01 / 09 / 89$ & 0.00 & $02 / 27 / 89$ & 11.51 \\
\hline $10 / 04 / 88$ & 0.00 & $11 / 22 / 88$ & 0.00 & $01 / 10 / 89$ & 2.75 & $02 / 28 / 89$ & 14.82 \\
\hline $10 / 05 / 88$ & 0.00 & $11 / 23 / 88$ & 0.00 & $01 / 11 / 89$ & 32.54 & $03 / 01 / 89$ & 0.00 \\
\hline $10 / 06 / 88$ & 0.00 & $11 / 24 / 88$ & 0.00 & $01 / 12 / 89$ & 45.49 & $03 / 02 / 89$ & 0.00 \\
\hline $10 / 07 / 88$ & 0.00 & $11 / 25 / 88$ & 0.00 & $01 / 13 / 89$ & 11.03 & $03 / 03 / 89$ & 0.00 \\
\hline $10 / 08 / 88$ & 0.00 & $11 / 26 / 88$ & 0.25 & $01 / 14 / 89$ & 21.71 & $03 / 04 / 89$ & 7.04 \\
\hline $10 / 09 / 88$ & 0.00 & $11 / 27 / 88$ & 25.65 & $01 / 15 / 89$ & 1.00 & $03 / 05 / 89$ & 30.65 \\
\hline $10 / 10 / 88$ & 0.00 & $11 / 28 / 88$ & 0.00 & $01 / 16 / 89$ & 0.00 & $03 / 06 / 89$ & 3.28 \\
\hline $10 / 11 / 88$ & 0.00 & $11 / 29 / 88$ & 0.00 & $01 / 17 / 89$ & 0.00 & $03 / 07 / 89$ & 0.00 \\
\hline $10 / 12 / 88$ & 0.00 & $11 / 30 / 88$ & 0.00 & $01 / 18 / 89$ & 0.00 & $03 / 08 / 89$ & 0.00 \\
\hline $10 / 13 / 88$ & 0.00 & $12 / 01 / 88$ & 0.00 & $01 / 19 / 89$ & 0.00 & $03 / 09 / 89$ & 0.00 \\
\hline $10 / 14 / 88$ & 0.00 & $12 / 02 / 88$ & 0.00 & $01 / 20 / 89$ & 0.00 & $03 / 10 / 89$ & 0.00 \\
\hline $10 / 15 / 88$ & 0.00 & $12 / 03 / 88$ & 0.00 & $01 / 21 / 89$ & 0.00 & $03 / 11 / 89$ & 0.00 \\
\hline $10 / 16 / 88$ & 0.00 & $12 / 04 / 88$ & 0.00 & $01 / 22 / 89$ & 0.00 & $03 / 12 / 89$ & 0.00 \\
\hline $10 / 17 / 88$ & 8.13 & $12 / 05 / 88$ & 0.00 & $01 / 23 / 89$ & 0.00 & $03 / 13 / 89$ & 0.00 \\
\hline $10 / 18 / 88$ & 0.00 & $12 / 06 / 88$ & 0.00 & $01 / 24 / 89$ & 0.00 & $03 / 14 / 89$ & 0.00 \\
\hline $10 / 19 / 88$ & 0.00 & $12 / 07 / 88$ & 0.00 & $01 / 25 / 89$ & 0.00 & $03 / 15 / 89$ & 1.51 \\
\hline $10 / 20 / 88$ & 2.03 & $12 / 08 / 88$ & 0.00 & $01 / 26 / 89$ & 1.50 & $03 / 16 / 89$ & 0.25 \\
\hline $10 / 21 / 88$ & 9.65 & $12 / 09 / 88$ & 10.41 & $01 / 27 / 89$ & 0.00 & $03 / 17 / 89$ & 0.00 \\
\hline $10 / 22 / 88$ & 0.00 & $12 / 10 / 88$ & 1.78 & $01 / 28 / 89$ & 0.00 & $03 / 18 / 89$ & 18.25 \\
\hline $10 / 23 / 88$ & 3.56 & $12 / 11 / 88$ & 0.00 & $01 / 29 / 89$ & 0.25 & $03 / 19 / 89$ & 0.00 \\
\hline $10 / 24 / 88$ & 0.25 & $12 / 12 / 88$ & 0.00 & $01 / 30 / 89$ & 14.11 & $03 / 20 / 89$ & 11.82 \\
\hline $10 / 25 / 88$ & 2.54 & $12 / 13 / 88$ & 0.00 & $01 / 31 / 89$ & 0.00 & $03 / 21 / 89$ & 11.62 \\
\hline $10 / 26 / 88$ & 0.00 & $12 / 14 / 88$ & 0.00 & $02 / 01 / 89$ & 0.00 & $03 / 22 / 89$ & 0.00 \\
\hline $10 / 27 / 88$ & 0.00 & $12 / 15 / 88$ & 0.00 & $02 / 02 / 89$ & 0.00 & $03 / 23 / 89$ & 12.25 \\
\hline $10 / 28 / 88$ & 7.37 & $12 / 16 / 88$ & 0.00 & $02 / 03 / 89$ & 2.01 & $03 / 24 / 89$ & 0.00 \\
\hline $10 / 29 / 88$ & 0.25 & $12 / 17 / 88$ & 0.00 & $02 / 04 / 89$ & 0.25 & $03 / 25 / 89$ & 0.25 \\
\hline $10 / 30 / 88$ & 1.02 & $12 / 18 / 88$ & 0.00 & $02 / 05 / 89$ & 15.61 & $03 / 26 / 89$ & 0.00 \\
\hline $10 / 31 / 88$ & 1.27 & $12 / 19 / 88$ & 0.00 & $02 / 06 / 89$ & 15.81 & $03 / 27 / 89$ & 0.00 \\
\hline $11 / 01 / 88$ & 0.00 & $12 / 20 / 88$ & 0.00 & $02 / 07 / 89$ & 2.77 & $03 / 28 / 89$ & 0.00 \\
\hline $11 / 02 / 88$ & 0.00 & $12 / 21 / 88$ & 18.80 & $02 / 08 / 89$ & 0.00 & $03 / 29 / 89$ & 0.25 \\
\hline $11 / 03 / 88$ & 0.00 & $12 / 22 / 88$ & 0.25 & 02/09/89 & 0.00 & $03 / 30 / 89$ & 29.93 \\
\hline $11 / 04 / 88$ & 41.91 & $12 / 23 / 88$ & 10.67 & $02 / 10 / 89$ & 0.00 & $03 / 31 / 89$ & 14.23 \\
\hline $11 / 05 / 88$ & 26.42 & $12 / 24 / 88$ & 14.73 & $02 / 11 / 89$ & 0.00 & $04 / 01 / 89$ & 0.00 \\
\hline $11 / 06 / 88$ & 1.02 & $12 / 25 / 88$ & 0.00 & $02 / 12 / 89$ & 0.00 & $04 / 02 / 89$ & 0.00 \\
\hline $11 / 07 / 88$ & 0.00 & $12 / 26 / 88$ & 0.00 & $02 / 13 / 89$ & 1.25 & $04 / 03 / 89$ & 4.26 \\
\hline $11 / 08 / 88$ & 2.29 & $12 / 27 / 88$ & 0.00 & $02 / 14 / 89$ & 10.09 & $04 / 04 / 89$ & 23.98 \\
\hline $11 / 09 / 88$ & 0.00 & $12 / 28 / 88$ & 8.13 & $02 / 15 / 89$ & 0.00 & $04 / 05 / 89$ & 0.00 \\
\hline $11 / 10 / 88$ & 7.11 & $12 / 29 / 88$ & 0.00 & $02 / 16 / 89$ & 5.00 & $04 / 06 / 89$ & 0.25 \\
\hline $11 / 11 / 88$ & 0.00 & $12 / 30 / 88$ & 16.51 & $02 / 17 / 89$ & 34.51 & $04 / 07 / 89$ & 3.25 \\
\hline $11 / 12 / 88$ & 1.52 & $12 / 31 / 88$ & 33.02 & $02 / 18 / 89$ & 8.01 & $04 / 08 / 89$ & 8.52 \\
\hline $11 / 13 / 88$ & 2.29 & $01 / 01 / 89$ & 1.52 & $02 / 19 / 89$ & 0.00 & $04 / 09 / 89$ & 0.00 \\
\hline $11 / 14 / 88$ & 0.25 & $01 / 02 / 89$ & 1.27 & $02 / 20 / 89$ & 14.67 & $04 / 10 / 89$ & 0.00 \\
\hline $11 / 15 / 88$ & 0.00 & $01 / 03 / 89$ & 1.27 & $02 / 21 / 89$ & 22.80 & $04 / 11 / 89$ & 0.00 \\
\hline $11 / 16 / 88$ & 12.19 & $01 / 04 / 89$ & 0.00 & $02 / 22 / 89$ & 0.00 & $04 / 12 / 89$ & 0.00 \\
\hline $11 / 17 / 88$ & 0.00 & $01 / 05 / 89$ & 0.76 & $02 / 23 / 89$ & 0.00 & $04 / 13 / 89$ & 0.00 \\
\hline $11 / 18 / 88$ & 0.00 & $01 / 06 / 89$ & 15.74 & $02 / 24 / 89$ & 0.00 & $04 / 14 / 89$ & 1.75 \\
\hline
\end{tabular}


Table A.3 (continued)

\begin{tabular}{|c|c|c|c|c|c|c|c|}
\hline Date & $\begin{array}{l}\text { Precipitation } \\
\text { (mm) }\end{array}$ & Date & $\begin{array}{l}\text { Precipitation } \\
(\mathrm{mm})\end{array}$ & Date & $\begin{array}{l}\text { Precipitation } \\
(\mathrm{mm})\end{array}$ & Date & $\begin{array}{l}\text { Precipitation } \\
\text { (mm) }\end{array}$ \\
\hline $04 / 15 / 89$ & 4.51 & $06 / 03 / 89$ & 0.00 & $07 / 22 / 89$ & 3.03 & $09 / 09 / 89$ & 0.00 \\
\hline $04 / 16 / 89$ & 0.00 & $06 / 04 / 89$ & 3.52 & $07 / 23 / 89$ & 6.85 & $09 / 10 / 89$ & 0.00 \\
\hline $04 / 17 / 89$ & 0.00 & 06/05/89 & 15.67 & $07 / 24 / 89$ & 0.25 & $09 / 11 / 89$ & 14.98 \\
\hline $04 / 18 / 89$ & 0.00 & 06/06/89 & 17.21 & $07 / 25 / 89$ & 0.25 & $09 / 12 / 89$ & 0.00 \\
\hline $04 / 19 / 89$ & 0.00 & 06/07/89 & 0.00 & $07 / 26 / 89$ & 0.00 & $09 / 13 / 89$ & 0.00 \\
\hline $04 / 20 / 89$ & 0.00 & 06/08/89 & 9.56 & $07 / 27 / 89$ & 0.00 & $09 / 14 / 89$ & 12.17 \\
\hline $04 / 21 / 89$ & 0.00 & 06/09/89 & 17.76 & $07 / 28 / 89$ & 3.05 & $09 / 15 / 89$ & 45.88 \\
\hline $04 / 22 / 89$ & 0.00 & 06/10/89 & 0.00 & $07 / 29 / 89$ & 0.00 & $09 / 16 / 89$ & 0.51 \\
\hline $04 / 23 / 89$ & 2.28 & $06 / 11 / 89$ & 0.00 & $07 / 30 / 89$ & 3.04 & $09 / 17 / 89$ & 0.00 \\
\hline $04 / 24 / 89$ & 0.75 & $06 / 12 / 89$ & 20.54 & $07 / 31 / 89$ & 2.02 & 09/18/89 & 0.00 \\
\hline $04 / 25 / 89$ & 0.00 & $06 / 13 / 89$ & 8.86 & $08 / 01 / 89$ & 31.45 & $09 / 19 / 89$ & 0.00 \\
\hline $04 / 26 / 89$ & 0.00 & $06 / 14 / 89$ & 1.75 & $08 / 02 / 89$ & 0.00 & $09 / 20 / 89$ & 0.00 \\
\hline $04 / 27 / 89$ & 0.25 & $06 / 15 / 89$ & 35.68 & 08/03/89 & 0.00 & $09 / 21 / 89$ & 0.00 \\
\hline $04 / 28 / 89$ & 0.25 & $06 / 16 / 89$ & 14.93 & $08 / 04 / 89$ & 0.25 & $09 / 22 / 89$ & 33.35 \\
\hline $04 / 29 / 89$ & 5.58 & 06/17/89 & 0.00 & 08/05/89 & 0.00 & 09/2.3/89 & 6.08 \\
\hline $04 / 30 / 89$ & 0.25 & $06 / 18 / 89$ & 0.00 & 08/06/89 & 0.25 & $09 / 24 / 89$ & 0.00 \\
\hline $05 / 01 / 89$ & 13.13 & 06/19/89 & 4.28 & 08/07/89 & 0.00 & $09 / 25 / 89$ & 23.22 \\
\hline 05/02/89 & 0.00 & $06 / 20 / 89$ & 35.93 & 08/08/89 & 0.00 & $09 / 26 / 89$ & 0.25 \\
\hline 05/03/89 & 0.00 & $06 / 21 / 89$ & 8.63 & 08/09/89 & 0.00 & $09 / 27 / 89$ & 0.00 \\
\hline $05 / 04 / 89$ & 5.75 & $06 / 22 / 89$ & 17.21 & $08 / 10 / 89$ & 0.00 & $09 / 28 / 89$ & 1.00 \\
\hline 05/05/89 & 45.84 & $06 / 23 / 89$ & 1.01 & $08 / 11 / 89$ & 0.00 & 09/29/89 & 15.55 \\
\hline 05/06/89 & 1.77 & $06 / 24 / 89$ & 0.00 & $08 / 12 / 89$ & 0.00 & 09/30/89 & 55.31 \\
\hline $05 / 07 / 89$ & 0.00 & $06 / 25 / 89$ & 0.00 & $08 / 13 / 89$ & 0.00 & $10 / 01 / 89$ & 9.31 \\
\hline 05/08/89 & 2.50 & $06 / 26 / 89$ & 0.00 & $08 / 14 / 89$ & 0.00 & $10 / 02 / 89$ & 0.25 \\
\hline 05/09/89 & 18.99 & $06 / 27 / 89$ & 0.00 & 08/15/89 & 1.25 & $10 / 03 / 89$ & 0.00 \\
\hline $05 / 10 / 89$ & 0.00 & $06 / 28 / 89$ & 8.83 & 08/16/89 & 0.00 & $10 / 04 / 89$ & 0.00 \\
\hline $05 / 11 / 89$ & 0.00 & $06 / 29 / 89$ & 0.00 & $08 / 17 / 89$ & 0.25 & $10 / 05 / 89$ & 0.00 \\
\hline $05 / 12 / 89$ & 0.00 & $06 / 30 / 89$ & 0.00 & $08 / 18 / 89$ & 0.25 & $10 / 06 / 89$ & 0.00 \\
\hline $05 / 13 / 89$ & 0.00 & $07 / 01 / 89$ & 5.04 & $08 / 19 / 89$ & 0.00 & $10 / 07 / 89$ & 0.00 \\
\hline $05 / 14 / 89$ & 0.00 & $07 / 02 / 89$ & 7.01 & $08 / 20 / 89$ & 0.00 & $10 / 08 / 89$ & 0.00 \\
\hline $05 / 15 / 89$ & 0.50 & $07 / 03 / 89$ & 21.45 & $08 / 21 / 89$ & 0.00 & $10 / 09 / 89$ & 0.00 \\
\hline $05 / 16 / 89$ & 0.00 & $07 / 04 / 89$ & 1.76 & $08 / 22 / 89$ & 0.00 & $10 / 10 / 89$ & 0.00 \\
\hline $05 / 17 / 89$ & 0.00 & 07/05/89 & 0.25 & $08 / 23 / 89$ & 30.16 & $10 / 11 / 89$ & 0.00 \\
\hline 05/18/89 & 0.00 & $07 / 06 / 89$ & 35.25 & $08 / 24 / 89$ & 2.50 & $10 / 12 / 89$ & 0.00 \\
\hline 05/19/89 & 2.25 & $07 / 07 / 89$ & 0.25 & $08 / 25 / 89$ & 0.00 & $10 / 13 / 89$ & 0.00 \\
\hline $05 / 20 / 89$ & 29.95 & $07 / 08 / 89$ & 0.00 & $08 / 26 / 89$ & 4.56 & $10 / 14 / 89$ & 0.00 \\
\hline $05 / 21 / 89$ & 0.00 & $07 / 09 / 89$ & 0.00 & $08 / 27 / 89$ & 0.00 & $10 / 15 / 89$ & 0.00 \\
\hline $05 / 22 / 89$ & 4.06 & 07/10/89 & 0.00 & 08/28/89 & 0.00 & $10 / 16 / 89$ & 17.70 \\
\hline $05 / 23 / 89$ & 0.50 & $07 / 11 / 89$ & 0.00 & $08 / 29 / 89$ & 0.00 & $10 / 17 / 89$ & 20.27 \\
\hline $05 / 24 / 89$ & 0.00 & $07 / 12 / 89$ & 4.02 & $08 / 30 / 89$ & 4.30 & $10 / 18 / 89$ & 7.80 \\
\hline 05/25/89 & 0.00 & $07 / 13 / 89$ & 1.26 & $08 / 31 / 89$ & 0.00 & $10 / 19 / 89$ & 0.25 \\
\hline $05 / 26 / 89$ & 15.97 & $07 / 14 / 89$ & 0.00 & $09 / 01 / 89$ & 30.98 & $10 / 20 / 89$ & 0.00 \\
\hline $05 / 27 / 89$ & 11.64 & $07 / 15 / 89$ & 0.00 & $09 / 02 / 89$ & 0.25 & $10 / 21 / 89$ & 0.00 \\
\hline $05 / 28 / 89$ & 0.00 & $07 / 16 / 89$ & 3.00 & 09/03/89 & 0.00 & $10 / 22 / 89$ & 0.00 \\
\hline 05/29/89 & 0.00 & $07 / 17 / 89$ & 0.00 & $09 / 04 / 89$ & 0.00 & $10 / 23 / 89$ & 0.00 \\
\hline $05 / 30 / 89$ & 0.00 & $07 / 18 / 89$ & 0.00 & $09 / 05 / 89$ & 0.00 & $10 / 24 / 89$ & 0.00 \\
\hline $05 / 31 / 89$ & 0.00 & $07 / 19 / 89$ & 7.36 & $09 / 06 / 89$ & 0.00 & $10 / 25 / 89$ & 0.00 \\
\hline 06/01/89 & 0.00 & $07 / 20 / 89$ & 0.25 & $09 / 07 / 89$ & 0.00 & $10 / 26 / 89$ & 0.00 \\
\hline $06 / 02 / 89$ & 0.00 & $07 / 21 / 89$ & 6.57 & 09/08/89 & 0.00 & $10 / 27 / 89$ & 0.00 \\
\hline
\end{tabular}


Table A.3 (continued)

\begin{tabular}{|c|c|c|c|c|c|c|c|}
\hline Date & $\begin{array}{l}\text { Precipitation } \\
(\mathrm{mm})\end{array}$ & Date & $\begin{array}{l}\text { Precipitation } \\
\text { (mm) }\end{array}$ & Date & $\begin{array}{c}\text { Precipitation } \\
(\mathrm{mm})\end{array}$ & Date & $\begin{array}{l}\text { Precipitation } \\
\text { (mm) }\end{array}$ \\
\hline $10 / 28 / 89$ & 0.00 & $12 / 16 / 89$ & 0.00 & $02 / 03 / 90$ & 57.00 & $03 / 24 / 90$ & 0.00 \\
\hline $10 / 29 / 89$ & 0.00 & $12 / 17 / 89$ & 0.00 & $02 / 04 / 90$ & 6.25 & $03 / 25 / 90$ & 0.00 \\
\hline $10 / 30 / 89$ & 0.00 & $12 / 18 / 89$ & 0.00 & $02 / 05 / 90$ & 0.00 & $03 / 26 / 90$ & 0.00 \\
\hline $10 / 31 / 89$ & 4.32 & $12 / 19 / 89$ & 6.25 & $02 / 06 / 90$ & 1.00 & $03 / 27 / 90$ & 0.00 \\
\hline $11 / 01 / 89$ & 0.00 & $12 / 20 / 89$ & 0.25 & $02 / 07 / 90$ & 2.25 & 03/28/90 & 0.00 \\
\hline $11 / 02 / 89$ & 0.00 & $12 / 21 / 89$ & 0.00 & $02 / 08 / 90$ & 0.00 & 03/29/90 & 4.25 \\
\hline $11 / 03 / 89$ & 0.00 & $12 / 22 / 89$ & 0.00 & $02 / 09 / 90$ & 21.25 & $03 / 30 / 90$ & 2.75 \\
\hline $11 / 04 / 89$ & 0.00 & $12 / 23 / 89$ & 0.00 & $02 / 10 / 90$ & 18.50 & $03 / 31 / 90$ & 0.50 \\
\hline $11 / 05 / 89$ & 0.00 & $12 / 24 / 89$ & 0.00 & $02 / 11 / 90$ & 0.00 & $04 / 01 / 90$ & 0.00 \\
\hline $11 / 06 / 89$ & 19.77 & $12 / 25 / 89$ & 0.00 & $02 / 12 / 90$ & 0.00 & $04 / 02 / 90$ & 0.00 \\
\hline $11 / 07 / 89$ & 16.24 & $12 / 26 / 89$ & 1.00 & $02 / 13 / 90$ & 0.00 & $04 / 03 / 90$ & 0.00 \\
\hline $11 / 08 / 89$ & 13.95 & $12 / 27 / 89$ & 0.00 & $02 / 14 / 90$ & 0.00 & $04 / 04 / 90$ & 0.00 \\
\hline $11 / 09 / 89$ & 1.25 & $12 / 28 / 89$ & 0.00 & $02 / 15 / 90$ & 18.50 & $04 / 05 / 90$ & 0.00 \\
\hline $11 / 10 / 89$ & 0.00 & $12 / 29 / 89$ & 1.00 & $02 / 16 / 90$ & 34.00 & $04 / 06 / 90$ & 0.00 \\
\hline $11 / 11 / 89$ & 0.00 & $12 / 30 / 89$ & 14.36 & $02 / 17 / 90$ & 0.00 & $04 / 07 / 90$ & 0.00 \\
\hline $11 / 12 / 89$ & 0.00 & $12 / 31 / 89$ & 29.35 & $02 / 18 / 90$ & 6.00 & $04 / 08 / 90$ & 0.00 \\
\hline $11 / 13 / 89$ & 0.00 & $01 / 01 / 90$ & 0.00 & $02 / 19 / 90$ & 5.25 & $04 / 09 / 90$ & 0.00 \\
\hline $11 / 14 / 89$ & 6.50 & $01 / 02 / 90$ & 0.00 & $02 / 20 / 90$ & 0.00 & $04 / 10 / 90$ & 0.00 \\
\hline $11 / 15 / 89$ & 35.67 & $01 / 03 / 90$ & 0.25 & $02 / 21 / 90$ & 0.00 & $04 / 11 / 90$ & 0.00 \\
\hline $11 / 16 / 89$ & 0.00 & $01 / 04 / 90$ & 10.25 & $02 / 22 / 90$ & 9.00 & $04 / 12 / 90$ & 0.00 \\
\hline $11 / 17 / 89$ & 0.00 & $01 / 05 / 90$ & 4.25 & $02 / 23 / 90$ & 0.00 & $04 / 13 / 90$ & 5.25 \\
\hline $11 / 18 / 89$ & 0.00 & $01 / 06 / 90$ & 4.00 & $02 / 24 / 90$ & 0.00 & $04 / 14 / 90$ & 0.25 \\
\hline $11 / 19 / 89$ & 0.00 & $01 / 07 / 90$ & 3.75 & $02 / 25 / 90$ & 0.00 & $04 / 15 / 90$ & 0.00 \\
\hline $11 / 20 / 89$ & 0.00 & $01 / 08 / 90$ & 13.25 & $02 / 26 / 90$ & 0.00 & $04 / 16 / 90$ & 5.01 \\
\hline $11 / 21 / 89$ & 0.00 & $01 / 09 / 90$ & 0.00 & $02 / 27 / 90$ & 0.00 & $04 / 17 / 90$ & 0.00 \\
\hline $11 / 22 / 89$ & 21.16 & $01 / 10 / 90$ & 0.50 & $02 / 28 / 90$ & 0.00 & $04 / 18 / 90$ & 0.25 \\
\hline $11 / 23 / 89$ & 0.25 & $01 / 11 / 90$ & 0.00 & $03 / 01 / 90$ & 2.25 & $04 / 19 / 90$ & 0.00 \\
\hline $11 / 24 / 89$ & 0.00 & $01 / 12 / 90$ & 0.00 & $03 / 02 / 90$ & 15.50 & $04 / 20 / 90$ & 0.00 \\
\hline $11 / 25 / 89$ & 0.00 & $01 / 13 / 90$ & 0.00 & $03 / 03 / 90$ & 1.00 & $04 / 21 / 90$ & 10.34 \\
\hline $11 / 26 / 89$ & 0.75 & $01 / 14 / 90$ & 0.00 & $03 / 04 / 90$ & 0.00 & $04 / 22 / 90$ & 0.00 \\
\hline $11 / 27 / 89$ & 2.02 & $01 / 15 / 90$ & 0.00 & $03 / 05 / 90$ & 0.00 & $04 / 23 / 90$ & 0.00 \\
\hline $11 / 28 / 89$ & 18.29 & $01 / 16 / 90$ & 0.00 & $03 / 06 / 90$ & 0.00 & $04 / 24 / 90$ & 0.00 \\
\hline $11 / 29 / 89$ & 0.00 & $01 / 17 / 90$ & 0.25 & $03 / 07 / 90$ & 0.00 & $04 / 25 / 90$ & 0.00 \\
\hline $11 / 30 / 89$ & 0.00 & $01 / 18 / 90$ & 19.00 & $03 / 08 / 90$ & 4.50 & $04 / 26 / 90$ & 0.00 \\
\hline $12 / 01 / 89$ & 0.00 & $01 / 19 / 90$ & 0.00 & $03 / 09 / 90$ & 3.27 & $04 / 27 / 90$ & 0.00 \\
\hline $12 / 02 / 89$ & 0.00 & $01 / 20 / 90$ & 30.25 & $03 / 10 / 90$ & 9.38 & $04 / 28 / 90$ & 22.01 \\
\hline $12 / 03 / 89$ & 0.00 & $01 / 21 / 90$ & 0.00 & $03 / 11 / 90$ & 0.00 & $04 / 29 / 90$ & 0.25 \\
\hline $12 / 04 / 89$ & 0.00 & $01 / 22 / 90$ & 0.00 & $03 / 12 / 90$ & 0.00 & $04 / 30 / 90$ & 0.00 \\
\hline $12 / 05 / 89$ & 0.00 & $01 / 23 / 90$ & 0.00 & $03 / 13 / 90$ & 0.00 & $05 / 01 / 90$ & 56.00 \\
\hline $12 / 06 / 89$ & 0.00 & $01 / 24 / 90$ & 8.75 & $03 / 14 / 90$ & 0.00 & $05 / 02 / 90$ & 0.00 \\
\hline $12 / 07 / 89$ & 3.00 & $01 / 25 / 90$ & 5.50 & $03 / 15 / 90$ & 2.00 & $05 / 03 / 90$ & 25.50 \\
\hline $12 / 08 / 89$ & 11.26 & $01 / 26 / 90$ & 0.00 & $03 / 16 / 90$ & 47.92 & $05 / 04 / 90$ & 13.00 \\
\hline $12 / 09 / 89$ & 0.75 & $01 / 27 / 90$ & 0.00 & $03 / 17 / 90$ & 16.38 & $05 / 05 / 90$ & 1.75 \\
\hline $12 / 10 / 89$ & 0.00 & $01 / 28 / 90$ & 0.00 & $03 / 18 / 90$ & 0.00 & $05 / 06 / 90$ & 0.50 \\
\hline $12 / 11 / 89$ & 2.25 & $01 / 29 / 90$ & 33.25 & $03 / 19 / 90$ & 3.50 & $05 / 07 / 90$ & 0.00 \\
\hline $12 / 12 / 89$ & 3.25 & $01 / 30 / 90$ & 0.00 & $03 / 20 / 90$ & 0.00 & $05 / 08 / 90$ & 0.00 \\
\hline $12 / 13 / 89$ & 0.00 & $01 / 31 / 90$ & 0.00 & $03 / 21 / 90$ & 0.00 & $05 / 09 / 90$ & 18.00 \\
\hline $12 / 14 / 89$ & 0.00 & $02 / 01 / 90$ & 0.00 & $03 / 22 / 90$ & 0.00 & $05 / 10 / 90$ & 4.00 \\
\hline $12 / 15 / 89$ & 0.25 & $02 / 02 / 90$ & 2.00 & $03 / 23 / 90$ & 0.00 & $05 / 11 / 90$ & 0.00 \\
\hline
\end{tabular}


Table A.3 (continued)

\begin{tabular}{|c|c|c|c|c|c|}
\hline Date & $\begin{array}{l}\text { Precipitation } \\
(\mathrm{mm})\end{array}$ & Date & $\begin{array}{c}\text { Precipitation } \\
(\mathrm{mm})\end{array}$ & Date & $\begin{array}{c}\text { Precipitation } \\
(\mathrm{mm})\end{array}$ \\
\hline $05 / 12 / 90$ & 0.00 & $06 / 30 / 90$ & 0.00 & $08 / 18 / 90$ & 0.00 \\
\hline 05/13/90 & 0.00 & $07 / 01 / 90$ & 34.04 & $08 / 19 / 90$ & 0.76 \\
\hline $05 / 14 / 90$ & 0.00 & $07 / 02 / 90$ & 0.00 & $08 / 20 / 90$ & 0.00 \\
\hline $05 / 15 / 90$ & 0.00 & $07 / 03 / 90$ & 0.00 & $08 / 21 / 90$ & 5.84 \\
\hline $05 / 16 / 90$ & 0.00 & $07 / 04 / 90$ & 0.00 & $08 / 22 / 90$ & 8.38 \\
\hline $05 / 17 / 90$ & 2.05 & $07 / 05 / 90$ & 0.00 & $08 / 23 / 90$ & 0.00 \\
\hline 05/18/90 & 0.00 & $07 / 06 / 90$ & 0.00 & $08 / 24 / 90$ & 0.00 \\
\hline $05 / 19 / 90$ & 0.00 & $07 / 07 / 90$ & 0.00 & $08 / 25 / 90$ & 0.00 \\
\hline $05 / 20 / 90$ & 7.00 & $07 / 08 / 90$ & 0.00 & $08 / 26 / 90$ & 0.00 \\
\hline $05 / 21 / 90$ & 1.00 & 07/09/90 & 0.00 & $08 / 27 / 90$ & 0.00 \\
\hline $05 / 22 / 90$ & 0.00 & $07 / 10 / 90$ & 0.00 & $08 / 28 / 90$ & 0.00 \\
\hline $05 / 23 / 90$ & 0.00 & $07 / 11 / 90$ & 24.64 & $08 / 29 / 90$ & 22.35 \\
\hline $05 / 24 / 90$ & 0.00 & $07 / 12 / 90$ & 50.04 & $08 / 30 / 90$ & 0.00 \\
\hline $05 / 25 / 90$ & 0.00 & $07 / 13 / 90$ & 25.91 & $08 / 31 / 90$ & 0.00 \\
\hline $05 / 26 / 90$ & 5.00 & $07 / 14 / 90$ & 14.22 & $09 / 01 / 90$ & 0.00 \\
\hline 05/27/90 & 14.50 & $07 / 15 / 90$ & 0.00 & $09 / 02 / 90$ & 0.00 \\
\hline $05 / 28 / 90$ & 20.50 & $07 / 16 / 90$ & 0.00 & $09 / 03 / 90$ & 0.00 \\
\hline $05 / 29 / 90$ & 0.00 & $07 / 17 / 90$ & 0.00 & $09 / 04 / 90$ & $0.0 ?$ \\
\hline $05 / 30 / 90$ & 0.00 & $07 ! 18 / 90$ & 0.00 & $09 / 05 / 90$ & 0.60 \\
\hline $05 / 31 / 90$ & 0.00 & $07 / 19 / 90$ & 5.33 & $09 / 06 / 90$ & 0.00 \\
\hline $06 / 01 / 90$ & 0.00 & $07 / 20 / 90$ & 0.00 & $09 / 07 / 90$ & 0.00 \\
\hline $06 / 02 / 90$ & 0.00 & $07 / 21 / 90$ & 28.19 & $09 / 08 / 90$ & 0.00 \\
\hline $06 / 03 / 90$ & 0.00 & $07 / 22 / 90$ & 5.84 & $09 / 09 / 90$ & 0.00 \\
\hline $06 / 04 / 90$ & 0.00 & $07 / 23 / 90$ & 0.00 & $09 / 10 / 90$ & 0.00 \\
\hline $06 / 05 / 90$ & 0.00 & $07 / 24 / 90$ & 0.00 & $09 / 11 / 90$ & 0.00 \\
\hline $06 / 06 / 90$ & 0.00 & $07 / 25 / 90$ & 0.00 & $09 / 12 / 90$ & 5.75 \\
\hline $06 / 07 / 90$ & 0.00 & $07 / 26 / 90$ & 0.00 & $09 / 13 / 90$ & 2.25 \\
\hline $06 / 08 / 90$ & 0.00 & $07 / 27 / 90$ & 0.00 & $09 / 14 / 90$ & 21.50 \\
\hline $06 / 09 / 90$ & 0.00 & $07 / 28 / 90$ & 0.00 & $09 / 15 / 90$ & 0.25 \\
\hline 06/10/90 & 0.00 & $07 / 29 / 90$ & 0.00 & $09 / 16 / 90$ & 0.00 \\
\hline $06 / 11 / 90$ & 0.00 & $07 / 30 / 90$ & 0.00 & $09 / 17 / 90$ & 0.00 \\
\hline $06 / 12 / 90$ & 0.00 & $07 / 31 / 90$ & 0.00 & $09 / 18 / 90$ & 0.00 \\
\hline 06/13/90 & 0.00 & $08 / 01 / 90$ & 0.00 & $09 / 19 / 90$ & 12.50 \\
\hline $06 / 14 / 90$ & 8.62 & $08 / 02 / 90$ & 0.00 & $09 / 20 / 90$ & 0.00 \\
\hline $06 / 15 / 90$ & 0.00 & $08 / 03 / 90$ & 0.00 & $09 / 21 / 90$ & 7.00 \\
\hline $06 / 16 / 90$ & 0.00 & $08 / 04 / 90$ & 21.59 & $09 / 22 / 90$ & 1.00 \\
\hline $06 / 17 / 90$ & 0.00 & $08 / 05 / 90$ & 22.61 & $09 / 23 / 90$ & 0.00 \\
\hline $06 / 18 / 90$ & 0.00 & $08 / 06 / 90$ & 3.56 & $09 / 24 / 90$ & 0.00 \\
\hline 06/19/90 & 0.00 & 08/07/90 & 0.00 & $09 / 25 / 90$ & 0.00 \\
\hline $06 / 20 / 90$ & 0.00 & 08/08/90 & 12.95 & $09 / 26 / 90$ & 0.00 \\
\hline $06 / 21 / 90$ & 1.00 & 08/09/90 & 18.29 & $09 / 27 / 90$ & 0.00 \\
\hline $06 / 22 / 90$ & 7.37 & $08 / 10 / 90$ & 0.00 & $09 / 28 / 90$ & 0.00 \\
\hline 06/23/90 & 0.00 & $08 / 11 / 90$ & 0.00 & $09 / 29 / 90$ & 0.00 \\
\hline $06 / 24 / 90$ & 0.00 & $08 / 12 / 90$ & 0.00 & $09 / 30 / 90$ & 0.50 \\
\hline $06 / 25 / 90$ & 0.00 & $08 / 13 / 90$ & 0.76 & & \\
\hline $06 / 26 / 90$ & 0.00 & $08 / 14 / 90$ & 13.72 & & \\
\hline $06 / 27 / 90$ & 0.00 & $08 / 15 / 90$ & 0.00 & & \\
\hline $06 / 28 / 90$ & 0.00 & $08 / 16 / 90$ & 0.00 & & \\
\hline $06 / 29 / 90$ & 0.76 & $08 / 17 / 90$ & 10.92 & & \\
\hline
\end{tabular}


Table A.4. Peak monthly elevations of White Oak Lake from October 1988 through September 1990

\begin{tabular}{ccc}
$\begin{array}{c}\text { Date peak } \\
\text { occurred }\end{array}$ & $\begin{array}{c}\text { Lake } \\
\text { elevation } \\
(\mathrm{ft})\end{array}$ & $\begin{array}{c}\text { Lake } \\
\text { elevation } \\
(\mathrm{m})\end{array}$ \\
\hline $10-02-88$ & 745.08 & 227.16 \\
$12-31-88$ & 747.44 & 227.88 \\
$01-12-89$ & 748.19 & 228.11 \\
$02-21-89$ & 747.28 & 227.83 \\
$03-30-89$ & 746.98 & 227.74 \\
$04-04-89$ & 746.41 & 227.56 \\
$05-05-89$ & 746.75 & 227.67 \\
$06-22-89$ & 747.11 & 227.78 \\
$07-06-89$ & 746.45 & 227.58 \\
$08-01-89$ & 745.72 & 227.35 \\
$09-30-89$ & 748.47 & 228.19 \\
$10-01-89$ & 748.65 & 228.25 \\
$11-16-89$ & 747.43 & 227.88 \\
$12-31-89$ & 747.78 & 227.98 \\
$01-29-90$ & 747.52 & 227.90 \\
$02-04-90$ & 748.08 & 228.07 \\
$03-17-90$ & 748.16 & 228.10 \\
$04-22-90$ & 745.56 & 227.30 \\
$05-01-90$ & 749.00 & 228.35 \\
$06-09-90$ & 745.78 & 227.37 \\
$07-14-90$ & 746.76 & 227.67 \\
$08-09-90$ & 746.90 & 227.71 \\
$09-15-90$ & 745.44 & 227.27 \\
\hline
\end{tabular}


APPENDIX B: Procedures for Soil Penetration Testing and Monitoring Well Installation in Radioactive Waste Trenches 


\section{PROCEDURES FOR SOIL PENETRATION TESTING AND MONITORING WELL INSTALLATION IN RADIOACTIVE WASTE TRENCHES}

\section{PURPOSE}

The purpose of this document is to describe procedures to be put into effect in the conducting of soil stability (penetration) tests and installation of monitoring wells in lowlevel radioactive waste trenches. The procedures are aimed specifically at the Solid Waste Storage Area (SWSA) 6 Test Area for Remedial Action (TARA) experimental site, which has a need to conduct such activities, but also has application at other Oak Ridge National Laboratory (ORNL) waste disposal sites where measuring soil cap stability and monitoring or sampling trench water may be deemed necessary.

\section{PROCEDURES}

1. All personnel within a $10-\mathrm{ft}$ radius of the trench-penetration point will wear contamination zone clothing, gloves, full-face respirator with combination cartridges, shoe covers, and hard hats. Respirators will be taken off only when radiation protection (RP) and industrial hygiene (IH) personnel have surveyed and determined that a particular hole is not contaminated and respiratory protection is no longer required.

2. Penetration of the trench will be achieved using the drop hammer of a trailer-mounted drill rig to drive a 1.75-in-diam steel drill rod into the trench cap and trench contents in much the same way that one would drive a nail into a piece of wood. The $140-1 b$ drop hammer will be physically connected to the drill rod at all times during hammering and the tip end of the drill rod will be fitted with a 2 -in. cone-shaped steel penetration point to facilitate driving into the soil. The maximum depth of penetration will be $15 \mathrm{ft}$, which includes three 5-ft sections of drill rod.

3. Before penetration starts at a particular hole, an -3 -ft-square piece of plastic will be laid out over the ground to serve as protection against the spread of radioactive coniamination should any be encountered during the process.

4. The drill rod will then be driven into the trench to the maximum depth of $15 \mathrm{ft}$, at which time the drill rig will be shut off and a radiation survey of the hole area will be made while the rod is still in the hole. If a hard piece of waste such as a steel container or concrete debris is encountered, the hammering will stop short of the desired $15 \mathrm{ft}$ depth and the resulting hole will either be filled with soil or cased with well screen material, depending on the depths of other holes in that particular trench. At least two 15- $\mathrm{ft}$ deep holes are desired in each burial trench to facilitate both grout injection and grout monitoring. The additional three holes in each trench can be of intermediate depth $(>5 \mathrm{ft}$ and $<15 \mathrm{ft})$.

5. Removal of the drill rod will be accomplished by reversing the "hammering in" process and using the hammer to bump the rod out of the hole. The suspect drill rod that is slowly emerging from the ground (inch by inch as the hammer bumps it out) will be pulled into a plastic sleeve that is attached at the bottom end to the $3 \mathrm{ft}$ by $3 \mathrm{ft}$ sheet of plastic covering the ground and at the top end near the connection to the clean drop hammer. In this manner, the suspect drill rod is never exposed to the air, avoiding the possible spread of contamination, should any be brought to the surface.

6. During the drill rod removal process, continuous beta-gamma monitoring of the rod will be accomplished with a GM probe attached to a meter stick and positioned at the 
ground surface so that the removal process can be stopped if it is determined that the rod is highly contaminated (see 7). If no contamination is detected by this preliminary survey, the rod will continue to be bumped out of the ground and into the plastic sleeve until $\sim 6 \mathrm{ft}$ of rod is above ground. At this point, the drill rig will be shut down and the HP will initiate a more detailed beta-gamma survey with the plastic sleeve in place.

7. If the rod is found to be contaminated by either the beta-gamma survey described in 6 , or by the alpha survey described in 8 , a decision will be made either to decontaminate the rod or to drive the rod back in the hole and sacrifice the drill rods. The decision level for hole and drill rod abandonment will be any beta-gamma surface reading $>10$ $\mathrm{mrad} / \mathrm{h}$.

8. Assuming the beta-gamma survey to show no contamination above the abandonment action level, the plastic sleeve will be cut at the top end where it is attached near the drop hammer and will be slowly pulled down the rod (an inch at a time) to facilitate an alpha activity survey. Again, if the rod is found to be contaminated, it will either be cleaned or driven back into the hole after resealing the plastic sleeve. The decision level for hole and drill rod abandonment will be an alpha surface reading exceeding $10,000 \mathrm{dpm} / 100 \mathrm{~cm}^{2}$.

9. Decontamination of a drill rod section will be initiated whenever surface survey instruments detect either: (a) alpha activity $>600 \mathrm{dpm} / 100 \mathrm{~cm}^{2}$ or (b) beta-gamma activity $>0.5 \mathrm{mrad} / \mathrm{h}$ on any length of drill rod section. Decontamination will consist of wiping the drill rod with moist cloth or paper towels by personnel in full protective clothing as described in 1, except that an additional pair of rubber gloves will be worn. Decontamination will continue until the on-site RP officer is satisfied that surficial activity is at or below the decontamination limits. If the drill rod cannot be decontaminated by this method, decontamination by scraping with a moistened wire brush and soap solution on paper towels will be attempted. If the rod cannot be decontaminated at this point, it will be designated as radioactive waste, packaged in plastic, and disposed by ORNL procedures.

10. After the top $5 \mathrm{ft}$ of drill rod is found to be clean, it will be removed and the drop hammer will be attached to the next 5 -ft section of drill rod to be extracted from the hole. Again, the plastic sleeve will be attached near the drop hammer and will still be attached at the ground surface so that this next piece of rod will also be drawn into a plastic sleeve. Monitoring for radioactivity will proceed as described herein, and this process will be repeated until all 3 sections of drill rod have been removed from the hole.

11. At this point, a 5-ft section of polyvinylchloride (PVC) well screen (with 0.1 -in. slots for grout wells or 0.01-in. slots for monitoring wells) will be inserted in the hole and gently tapped into place using the weight of the drop hammer. Additional well casing will be attached to the screen in $2 \mathrm{ft}$ sections and driven into the hole until the entire depth of the hole is cased. All casing protruding from the ground will be solid pipe as opposed to slotted well screen used for the remainder of the hole. A cap will be screwed onto the top of the PVC well, and IH will survey the hole for volatile organics. A penetration hole will be considered clean if the reading with a calibrated TIP Organic Vapor Analyzer is $<100$ units; the sensitivity of the TIP instrument will be set so that a reading of 1000 units is achieved with a standard of $100 \mathrm{ppm}$ isobutylene in air and a reading of 0 units is achieved in ambient air. If the hole is clean at this point, respirators will be removed and the drill rig will be moved to the site of the next hole. 
12. Immediately before leaving the well, a GM tube will be lowered down the dry cased hole to determine if there is extremely high radioactivity underground in proximity to the hole. This information, along with the $\mathrm{IH}$ gas analysis, will be valuable in predicting what hazards might exist in future water level monitoring or sampling of the well.

13. The well will be labeled with a metal tag identifying the trench number, for example T-199. Engineering will be contacted and requested to conduct an as built survey of location and elevation so that the wells can be placed on SWSA 6 maps.

14. Following completion of all penetration testing, the drill rig and any tools used in the testing will be thoroughly checked by RP personnel for any residual contamination. Decontamination of any tools or drill rig parts will be initiated whenever transferable smear activities exceed either $30 \mathrm{dpm} / 100 \mathrm{~cm}^{2}$ (alpha) or $200 \mathrm{dpm} / 100 \mathrm{~cm}^{2}$ (betagamma). 
APPENDIX C: Penetration Resistance of SWSA 6 Burial Trenches and Control Sites Úsed for Leachate Monitoring Wells 

Table C.1. Penetration resistiance (1 to $3 \mathrm{ft}$ ) of SWSA 6 burial trenches
and control sites used for leachate monitoring wells

\begin{tabular}{|c|c|c|c|c|c|c|}
\hline \multirow{2}{*}{$\begin{array}{l}\text { Trench } \\
\text { No. }\end{array}$} & \multirow{2}{*}{$\begin{array}{l}\text { Well } \\
\text { No. }\end{array}$} & \multirow{2}{*}{$\begin{array}{l}\text { Test } \\
\text { date }\end{array}$} & \multirow{2}{*}{$\begin{array}{l}\text { Test } \\
\text { time }\end{array}$} & \multicolumn{3}{|c|}{ Cumulative blow counts } \\
\hline & & & & $1 \mathrm{ft}$ & $2 \mathrm{ft}$ & $3 \mathrm{ft}$ \\
\hline 103 & T103B & $10-0 c t-88$ & $11: 08$ & 14 & 33 & 39 \\
\hline 103 & T103A & $10-0 c t-88$ & $13: 10$ & 8 & 20 & 30 \\
\hline 380 & None & $10-0 c t-88$ & $14: 00$ & 8 & 13 & 15 \\
\hline 380 & T380 & $10-0 c t-88$ & $14: 20$ & 7 & 12 & 16 \\
\hline 370 & T370 & $10-$ Oct- 88 & $14: 48$ & 17 & 41 & 65 \\
\hline 370 & None & $10-0 c t-88$ & $15: 15$ & 21 & 46 & 60 \\
\hline 370 & None & $10-0 c t-88$ & $15: 45$ & 19 & 44 & 58 \\
\hline 82 & T82 & $11-0 c t-88$ & $10: 33$ & 12 & 26 & 32 \\
\hline 120 & $\mathrm{~T} 120$ & $11-0 c t-88$ & $11: 00$ & 8 & 18 & 26 \\
\hline 408 & None & $11-0 c t-8 \varepsilon$ & $14: 55$ & 6 & 11 & 14 \\
\hline 408 & None & $11-$ Oct -88 & $15: 15$ & 6 & 11 & 14 \\
\hline 408 & T408 & $11-0 c t-88$ & $15: 30$ & 6 & 9 & 11 \\
\hline 397 & T397 & $12-0 c t-88$ & $09: 00$ & 9 & 24 & 32 \\
\hline 330 & T330 & $12-0 c t-88$ & $09: 30$ & 9 & 20 & 30 \\
\hline 363 & None & $12-0 c t-88$ & $10: 20$ & 16 & 28 & 34 \\
\hline 363 & None & $12-0 c t-88$ & $10: 50$ & 14 & 25 & 31 \\
\hline 363 & None & $12-0 c t-88$ & $11: 05$ & 15 & 28 & 37 \\
\hline 363 & T363 & $12-0 c t-88$ & $11: 15$ & 15 & 27 & 33 \\
\hline 69 & T69 & $12-0 c t-88$ & $13: 00$ & 19 & 36 & 41 \\
\hline 71 & None & $12-0 c t-88$ & $13: 30$ & 10 & 19 & 21 \\
\hline 71 & None & $12-0 c t-88$ & $13: 50$ & 15 & 25 & 31 \\
\hline 71 & None & $12-0 c t-88$ & $14: 05$ & 12 & 23 & 27 \\
\hline 71 & None & $12-0 c t-88$ & $14: 20$ & 32 & 64 & 92 \\
\hline 71 & None & $12-0 c t-88$ & $14: 30$ & 12 & 21 & 29 \\
\hline 71 & None & $12-0 c t-88$ & $14: 40$ & 13 & 21 & 24 \\
\hline 121 & $\mathrm{~T} 121$ & $12-0 c t-88$ & $15: 00$ & 8 & 17 & 31 \\
\hline 84 & None & $12-0 c t-88$ & $15: 30$ & 16 & 37 & 47 \\
\hline 84 & T84 & $13-0 c t-88$ & $09: 30$ & 13 & 32 & 41 \\
\hline 308 & T308 & $13-0 c t-88$ & $10: 00$ & 32 & 46 & 52 \\
\hline 308 & None & $13-0 c t-88$ & $10: 33$ & 20 & 36 & 46 \\
\hline 318 & T318 & $13-0 c t-88$ & $11: 00$ & 22 & 42 & 51 \\
\hline 112 & T112 & $13-0 c t-88$ & $14: 00$ & 14 & 25 & 32 \\
\hline 85 & $\mathrm{~T} 85$ & $13-0 c t-88$ & $14: 36$ & 11 & 20 & 29 \\
\hline 110 & T110 & $13-0 c t-88$ & $15: 21$ & 12 & 21 & 25 \\
\hline 105 & T105 & $14-0 c t-88$ & $09: 45$ & 12 & 26 & 32 \\
\hline 426 & T426 & $14-0 c t-88$ & $10: 20$ & 14 & 25 & 32 \\
\hline 432 & T432 & $14-0 c t-88$ & $11: 00$ & 6 & 12 & 16 \\
\hline 414 & T414 & $14-0 c t-88$ & $13: 00$ & 6 & 12 & 15 \\
\hline 413 & $\mathrm{~T} 413$ & $17-0 c t-88$ & $13: 30$ & 9 & 18 & 25 \\
\hline 395 & T395 & $17-0 c t-88$ & $14: 00$ & 9 & 20 & 26 \\
\hline 329 & T329 & $17-0 c t-88$ & $14: 35$ & 8 & 23 & 31 \\
\hline 79 & None & $17-0 c t-88$ & $14: 55$ & 8 & 16 & 24 \\
\hline 79 & T79 & $17-0 c t-88$ & $15: 10$ & 9 & 26 & 40 \\
\hline 101 & $\mathrm{~T} 101$ & $17-0 c t-88$ & $15: 25$ & 14 & 31 & 38 \\
\hline 461 & $\mathrm{~T} 461$ & $17-0 c t-88$ & $15: 40$ & 10 & 18 & 23 \\
\hline 424 & None & $18-0 c t-88$ & $09: 15$ & 9 & 17 & 23 \\
\hline
\end{tabular}




\begin{tabular}{|c|c|c|c|c|c|c|}
\hline \multirow{2}{*}{$\begin{array}{l}\text { Trench } \\
\text { No. }\end{array}$} & \multirow{2}{*}{$\begin{array}{l}\text { We11 } \\
\text { No. }\end{array}$} & \multirow{2}{*}{$\begin{array}{l}\text { Test } \\
\text { date }\end{array}$} & \multirow{2}{*}{$\begin{array}{l}\text { Test } \\
\text { time }\end{array}$} & \multicolumn{3}{|c|}{ Cumulative blow counts } \\
\hline & & & & $1 f t$ & $2 \mathrm{ft}$ & $3 \mathrm{ft}$ \\
\hline 424 & $\mathrm{~T} 424$ & $18-0 c t-88$ & $09: 30$ & 12 & 22 & 28 \\
\hline 373 & None & $18-0 c t-88$ & $10: 10$ & 5 & 9 & 17 \\
\hline 373 & T373 & $18-0 c t-88$ & $10: 45$ & 5 & 10 & 19 \\
\hline 352 & T352 & $18-0 c t-88$ & $13: 15$ & 5 & 15 & 22 \\
\hline 367 & T367 & $18-0 c t-88$ & $13: 50$ & 12 & 24 & 29 \\
\hline 453 & $\mathrm{~T} 453$ & $18-0 c t-88$ & $14: 30$ & 9 & 17 & 21 \\
\hline 4.22 & T422 & $18-0 c t-88$ & $15: 00$ & 19 & 33 & 38 \\
\hline 374 & T374 & $18-0 c t-88$ & $15: 38$ & 15 & 29 & 38 \\
\hline 241 & T241 & $19-$ Oct -88 & $09: 05$ & 11 & 23 & 30 \\
\hline 237 & T237 & $19-0 c t-88$ & $09: 35$ & 9 & 17 & 20 \\
\hline 180 & T180 & $19-0 c t-88$ & $10: 35$ & 12 & 24 & 29 \\
\hline 162 & $\mathrm{~T} 162$ & $19-0 c t-88$ & $13: 30$ & 9 & 20 & 26 \\
\hline 60 & $\mathrm{~T} 60$ & $19-0 c t-88$ & $14: 00$ & 12 & 24 & 31 \\
\hline 57 & None & $19-0 c t-88$ & $14: 30$ & 14 & 25 & 31 \\
\hline 57 & $\mathrm{~T} 57$ & $19-0 c t-88$ & $14: 40$ & 14 & 32 & 44 \\
\hline 44 & $\mathrm{~T} 44$ & $20-0 c t-88$ & & 10 & 19 & 23 \\
\hline 63 & T63 & $20-0 c t-88$ & & 11 & 23 & 30 \\
\hline 225 & $\mathrm{~T} 225$ & $20-0 c t-88$ & & 12 & 22 & 27 \\
\hline 279 & T279-6 & 08 -Aug - 89 & & 11 & 22 & 26 \\
\hline 288 & T288-6 & 08 -Aug- 89 & & 5 & 9 & 12 \\
\hline 13 & T13-6 & 08-Aug- 89 & & 20 & 29 & 33 \\
\hline 279 & T279-1 & $06-\mathrm{Feb}-90$ & & 5 & 12 & 16 \\
\hline 279 & T279-2 & $06-\mathrm{Feb}-90$ & & 5 & 10 & 15 \\
\hline 279 & T279-3 & $06-\mathrm{Feb}-90$ & & 6 & 10 & 13 \\
\hline 279 & T279-4 & $06-\mathrm{Feb}-90$ & & 4 & 9 & 12 \\
\hline 279 & T279-5 & $06-$ Feb- 90 & & 4 & 8 & 11 \\
\hline 288 & T288-1 & $08-\mathrm{Feb}-90$ & & 4 & 8 & 11 \\
\hline 288 & T288-2 & $08-F a b,-90$ & & 6 & 13 & 15 \\
\hline 288 & T288-3 & $08-\mathrm{Feb}-90$ & & 5 & 11 & 14 \\
\hline 288 & T288-4 & $08-F e b-90$ & & 4 & 9 & 12 \\
\hline 288 & T288-5 & $08-F e b-90$ & & 2 & 3 & 4 \\
\hline 288 & $\mathrm{~T} 288 \mathrm{~N}$ & $08-F e b-90$ & & 6 & 10 & 14 \\
\hline 13 & T13-5 & $13-F 、 b-90$ & & 5 & 11 & 17 \\
\hline 13 & T13-4 & $13-$ Feb- 90 & & 6 & 10 & 12 \\
\hline 13 & T13-3 & $13-\mathrm{Feb}-90$ & & 8 & 14 & 19 \\
\hline 13 & T13-2 & $13-\mathrm{Feb}-90$ & & 7 & 19 & 25 \\
\hline 13 & T13-1 & $13-$ Feb- 90 & & 7 & 14 & 17 \\
\hline 11 & $\mathrm{~T} 11$ & $13-\mathrm{Feb}-90$ & & 6 & 13 & 16 \\
\hline 16 & T16 & $13-\mathrm{Feb}-90$ & & 7 & 13 & 17 \\
\hline 252 & $\mathrm{~T} 252$ & $14-\mathrm{Feb}-90$ & & 9 & 16 & 20 \\
\hline 285 & $\mathrm{~T} 285$ & $14-F e b-90$ & & 5 & 9 & 11 \\
\hline 284 & T284 & $14-\mathrm{Feb}-90$ & & 4 & 7 & 8 \\
\hline 275 & $\mathrm{~T} 275$ & $14-\mathrm{Feb}-90$ & & 5 & 12 & 14 \\
\hline
\end{tabular}


Table C.1 (continued)

\begin{tabular}{|c|c|c|c|c|c|c|}
\hline \multirow{2}{*}{$\begin{array}{l}\text { Trench } \\
\text { No. }\end{array}$} & \multirow{2}{*}{$\begin{array}{l}\text { We11 } \\
\text { No. }\end{array}$} & \multirow{2}{*}{$\begin{array}{l}\text { Test } \\
\text { date }\end{array}$} & \multirow{2}{*}{$\begin{array}{l}\text { Tesi } \\
\text { time }\end{array}$} & \multicolumn{3}{|c|}{ Cumulative blow counts } \\
\hline & & & & Ift & $2 \mathrm{ft}$ & $3 \mathrm{ft}$ \\
\hline \multicolumn{7}{|c|}{ Wells near but outside burial trenches } \\
\hline 279 & T279N & $06-$ Feb- 90 & & 9 & 22 & 37 \\
\hline 279 & T279S & $06-\mathrm{Feb}-90$ & & 7 & 16 & 31 \\
\hline 279 & T279NE & $08-\mathrm{Feb}-90$ & & 6 & 13 & 23 \\
\hline 279 & T279SE & $08-\mathrm{Feb}-90$ & & 6 & 14 & 32 \\
\hline 279 & T279NW & $08-\mathrm{Feb}-90$ & & 6 & 16 & 25 \\
\hline 279 & T279SW & $08-\mathrm{Feb}-90$ & & 6 & 13 & 25 \\
\hline 288 & T288S & $12-\mathrm{Feb}-90$ & & 8 & 15 & 31 \\
\hline 288 & $\mathrm{~T} 288 \mathrm{NW}$ & $12-\mathrm{Feb}-90$ & & 9 & 17 & 31 \\
\hline 288 & $\mathrm{~T} 288 \mathrm{SW}$ & $12-$ Feb- 90 & & 7 & 13 & 32 \\
\hline 288 & $\mathrm{~T} 288 \mathrm{NE}$ & $12-\mathrm{Feb}-90$ & & 9 & 18 & 37 \\
\hline 288 & $\mathrm{~T} 288 \mathrm{SE}$ & $1.2-$ Feb- 90 & & 7 & 13 & 32 \\
\hline 13 & T13SE & $12-\mathrm{Feb}-90$ & & 8 & 27 & 55 \\
\hline 13 & T13NE & $12-F e b-90$ & & 6 & 19 & 50 \\
\hline 13 & T13S & $13-\mathrm{Feb}-90$ & & 8 & 13 & 24 \\
\hline 13 & $T^{2} 3 \mathrm{~N}$ & $13-\mathrm{Feb}-90$ & & 6 & 10 & 31 \\
\hline 13 & T13SW & $13-\mathrm{Feb}-90$ & & 6 & 23 & 43 \\
\hline 13 & T13NW & $13-\mathrm{Feb}-90$ & & 7 & 20 & 43 \\
\hline 151 & $\mathrm{~T} 151 \mathrm{E}$ & $05-F e b-90$ & & 4 & 8 & 17 \\
\hline 151 & T151SE & $05-$ Feb- 90 & & 6 & 17 & 27 \\
\hline 151 & T151S & $05-F e b-90$ & & 6 & 11 & 20 \\
\hline 151 & T151SW & $05-$ Feb-: 0 & & 6 & 10 & 20 \\
\hline 151 & T151NE & $05-$ Feb- 90 & & 4 & 7 & 19 \\
\hline 151 & $\mathrm{~T} 151 \mathrm{NW}$ & $05-$ Feb- 90 & & 5 & 9 & 23 \\
\hline 170 & T170NW & $05-$ Feb- 90 & & 3 & 6 & 9 \\
\hline 170 & T170W & $05-$ Feb- 90 & & 7 & 11 & 25 \\
\hline 151 & T151W & $05-$ Feb- 90 & & 6 & 10 & 25 \\
\hline
\end{tabular}


Table C.2. Penetration resistance ( 4 to $9 \mathrm{ft}$ ) of SWSA 6 burial trenches and control sites used for leachate monitoring wells

\begin{tabular}{|c|c|c|c|c|c|c|c|}
\hline \multirow{2}{*}{$\begin{array}{l}\text { Trench } \\
\text { No. }\end{array}$} & \multirow{2}{*}{$\begin{array}{l}\text { We11 } \\
\text { No. }\end{array}$} & \multicolumn{6}{|c|}{ Cumulaticve blow counts } \\
\hline & & $4 \mathrm{ft}$ & $5 \mathrm{ft}$ & $6 \mathrm{ft}$ & $7 \mathrm{ft}$ & $8 \mathrm{ft}$ & $9 \mathrm{ft}$ \\
\hline 103 & T103B & 43 & 47 & 49 & 51 & 53 & 56 \\
\hline 103 & T103A & 36 & 40 & 45 & 47 & 50 & 53 \\
\hline 380 & None & 18 & 21 & 37 & 46 & 51 & 55 \\
\hline 380 & T380 & 20 & 23 & 24 & 27 & 29 & 32 \\
\hline 370 & T370 & 75 & 84 & 91 & 99 & 105 & 116 \\
\hline 370 & None & 71 & 81 & 89 & 97 & 101 & 107 \\
\hline 370 & None & 64 & 78 & 90 & 99 & 104 & 109 \\
\hline 82 & $\mathrm{~T} 82$ & 36 & 39 & 41 & 43 & 45 & 47 \\
\hline 120 & T120 & 34 & 41 & 47 & 52 & 55 & 57 \\
\hline 408 & None & 16 & 18 & 20 & 22 & 24 & 26 \\
\hline 408 & None & 17 & 19 & 22 & 23 & 25 & 29 \\
\hline 408 & T408 & 13 & 14 & 16 & 19 & 20 & 22 \\
\hline 397 & T397 & 37 & 41 & 43 & 45 & 46 & 47 \\
\hline 330 & T330 & 42 & 52 & 59 & 62 & 65 & 68 \\
\hline 363 & None & 40 & 46 & 51 & 53 & 56 & 60 \\
\hline 363 & None & 36 & 41 & 45 & 48 & 50 & \\
\hline 363 & None & 42 & 47 & 52 & 54 & 57 & 58 \\
\hline 363 & T363 & 36 & 40 & 42 & 44 & 47 & 49 \\
\hline 69 & T69 & 43 & 49 & 55 & 66 & 74 & 89 \\
\hline 71 & None & 21 & 21 & 22 & 23 & 24 & 45 \\
\hline 71 & None & 54 & & & & & \\
\hline 71 & None & 28 & 29 & 32 & 33 & 34 & \\
\hline 71 & None & & & & & & \\
\hline 71 & None & 36 & & & & & \\
\hline 71 & None & 29 & 34 & 41 & 44 & 50 & 56 \\
\hline 121 & $\mathrm{~T} 121$ & 40 & 43 & 45 & 47 & 49 & 51 \\
\hline 84 & None & 53 & 58 & 61 & 65 & 69 & 74 \\
\hline 84 & T84 & 48 & 52 & 55 & 58 & 61 & 64 \\
\hline 308 & T308 & 55 & 56 & 58 & 61 & 71 & 86 \\
\hline 308 & None & 55 & 62 & 67 & 71 & 80 & 131 \\
\hline 318 & T318 & 57 & 59 & 61 & 64 & 66 & 88 \\
\hline 112 & T112 & 36 & 39 & 40 & 41 & 42 & 52 \\
\hline 85 & T85 & 41 & 49 & 55 & 62 & 69 & 77 \\
\hline 110 & T110 & 27 & 29 & 31 & 34 & 50 & 70 \\
\hline 105 & T105 & 37 & 39 & 40 & 42 & 44 & 59 \\
\hline 426 & T426 & 36 & 43 & 46 & 48 & 50 & 52 \\
\hline 432 & T432 & 18 & 21 & 25 & 27 & 31 & 35 \\
\hline 414 & T414 & 18 & 20 & 22 & 28 & 35 & 56 \\
\hline 413 & T413 & 28 & 31 & 32 & 34 & 36 & 48 \\
\hline 395 & T395 & 30 & 33 & 34 & 38 & 62 & 79 \\
\hline 329 & T329 & 35 & 39 & 40 & 44 & 48 & 53 \\
\hline 79 & None & 43 & 92 & & & & \\
\hline 79 & T79 & 58 & 88 & & & & \\
\hline 101 & $\mathrm{~T} 101$ & 42 & 47 & 50 & 54 & 57 & 88 \\
\hline 461 & T461 & 27 & 33 & 40 & 46 & 51 & 71 \\
\hline 424 & None & & & & & & \\
\hline 424 & T424 & 32 & 34 & 37 & 39 & 46 & 63 \\
\hline
\end{tabular}


Table C.2 (continued)

\begin{tabular}{|c|c|c|c|c|c|c|c|}
\hline \multirow{2}{*}{$\begin{array}{l}\text { Trench } \\
\text { No. }\end{array}$} & \multirow{2}{*}{$\begin{array}{c}\text { Well } \\
\text { No. }\end{array}$} & \multicolumn{6}{|c|}{ Gumulaticve blow counts } \\
\hline & & $4 \mathrm{ft}$ & $5 \mathrm{ft}$ & $6 \mathrm{ft}$ & $7 \mathrm{ft}$ & $8 \mathrm{ft}$ & $9 \mathrm{ft}$ \\
\hline 373 & None & 27 & 35 & 41 & 46 & 50 & 54 \\
\hline 373 & T373 & 31 & 40 & 46 & 51 & 56 & 60 \\
\hline 352 & T352 & 25 & 28 & 30 & 32 & 34 & 37 \\
\hline 367 & T367 & 32 & 36 & 38 & 39 & 42 & 51 \\
\hline 453 & T453 & 23 & 27 & 29 & 31 & 41 & 61 \\
\hline 422 & T422 & 40 & 43 & 51 & 53 & 63 & 90 \\
\hline 374 & T374 & 41 & 43 & 45 & 47 & 48 & 50 \\
\hline 241 & T241 & 35 & 38 & 41 & 42 & 44 & 45 \\
\hline 237 & T237 & 23 & 25 & 28 & 29 & 31 & 32 \\
\hline 180 & $\mathrm{~T} 180$ & 34 & 37 & 39 & 41 & 43 & 45 \\
\hline 162 & $\mathrm{~T} 162$ & 31 & 33 & 35 & 36 & 39 & 41 \\
\hline 60 & $\mathrm{~T} 60$ & 34 & 38 & 40 & 41 & 43 & 45 \\
\hline 57 & None & 36 & 49 & 82 & & & \\
\hline 57 & T57 & 49 & 54 & 56 & 58 & 59 & 61 \\
\hline 44 & T44 & 24 & 26 & 27 & 28 & 29 & 30 \\
\hline 63 & T63 & 34 & 36 & 38 & 39 & 42 & 44 \\
\hline 225 & T225 & 31 & 33 & 36 & 38 & 40 & 42 \\
\hline 279 & T279- 6 & 28 & 29 & 30 & 32 & 38 & 40 \\
\hline 288 & T288-6 & 14 & 15 & 17 & 18 & 19 & 28 \\
\hline 13 & T13-6 & 36 & 38 & 40 & 41 & 42 & 45 \\
\hline 279 & T279-1 & 18 & 19 & 20 & 21 & 22 & 43 \\
\hline 279 & $\mathrm{~T} 279-2$ & 18 & 20 & 22 & 24 & 26 & 34 \\
\hline 279 & T279-3 & 15 & 17 & 18 & 19 & 21 & 39 \\
\hline 279 & T279-4 & 14 & 16 & 18 & 19 & 20 & 31 \\
\hline 279 & T279-5 & 13 & 14 & 15 & 16 & 28 & 42 \\
\hline 288 & T288-1 & 13 & 15 & 16 & 21 & 24 & 26 \\
\hline 288 & T288-2 & 16 & 17 & 19 & 20 & 21 & 22 \\
\hline 288 & T288- 3 & 17 & 19 & 21 & 23 & 25 & 27 \\
\hline 288 & T288-4 & 13 & 15 & 22 & 25 & 28 & 30 \\
\hline 288 & T288-5 & 5 & 6 & 16 & 45 & 98 & 116 \\
\hline 288 & T288N & 20 & 24 & 25 & 29 & 32 & 53 \\
\hline 13 & T13-5 & 19 & 20 & 21 & 22 & 2.4 & 26 \\
\hline 13 & T13-4 & 14 & 16 & 18 & 19 & 20 & 21 \\
\hline 13 & T13-3 & 22 & 24 & 25 & 26 & 27 & 28 \\
\hline 13 & T13-2 & 28 & 30 & 31 & 32 & 33 & 34 \\
\hline 13 & T13-1 & 19 & 20 & 22 & 23 & 25 & 34 \\
\hline 11 & $\mathrm{~T} 11$ & 17 & 18 & 22 & 27 & 42 & 55 \\
\hline 16 & T16 & 19 & 20 & 21 & 23 & 24 & 36 \\
\hline 252 & T252 & 23 & 24 & 27 & 27 & 29 & 31 \\
\hline 285 & T285 & 13 & 14 & 19 & 32 & 53 & 91 \\
\hline 284 & T284 & 10 & 11 & 20 & 37 & 55 & 97 \\
\hline 275 & T275 & 15 & 24 & 36 & 59 & 73 & 93 \\
\hline
\end{tabular}


Table C.2 (continued)

\begin{tabular}{|c|c|c|c|c|c|c|c|}
\hline \multirow{2}{*}{$\begin{array}{l}\text { Trench } \\
\text { No. }\end{array}$} & \multirow{2}{*}{$\begin{array}{l}\text { Well } \\
\text { No. }\end{array}$} & \multicolumn{6}{|c|}{ Cumulaticve blow counts } \\
\hline & & $4 \mathrm{ft}$ & $5 \mathrm{ft}$ & $6 \mathrm{ft}$ & $7 \mathrm{ft}$ & $8 \mathrm{ft}$ & $9 \mathrm{ft}$ \\
\hline \multicolumn{8}{|c|}{ Wells near but outside burial trenches } \\
\hline 279 & T279N & 62 & 87 & 122 & 158 & 188 & 262 \\
\hline 279 & T279S & 66 & 108 & 146 & 223 & 289 & 332 \\
\hline 279 & T279NE & 41 & 63 & 101 & 145 & 175 & 241 \\
\hline 279 & T279SE & 53 & 88 & 140 & 202 & 248 & 313 \\
\hline 279 & $\mathrm{~T} 279 \mathrm{NW}$ & 46 & 89 & 138 & 169 & 195 & 239 \\
\hline 279 & T279SW & 57 & 89 & 134 & 165 & 205 & 228 \\
\hline 288 & $\mathrm{~T} 288 \mathrm{~S}$ & 70 & 118 & 176 & 232 & 296 & 343 \\
\hline 288 & $\mathrm{~T} 288 \mathrm{NW}$ & 56 & 75 & 94 & 141 & 186 & 223 \\
\hline 288 & $\mathrm{~T} 288 \mathrm{SW}$ & 49 & 75 & 104 & 135 & 170 & 218 \\
\hline 288 & T288NE & 59 & 89 & 115 & 134 & 157 & 220 \\
\hline 288 & T288SE & 61 & 86 & 121 & 148 & 182 & 216 \\
\hline 13 & T13SE & 77 & 128 & 179 & 227 & 255 & 283 \\
\hline 13 & T13NE & 89 & 118 & 155 & 188 & 210 & 254 \\
\hline 13 & T13S & 64 & 117 & 154 & 196 & 249 & 302 \\
\hline 13 & $\mathrm{~T} 13 \mathrm{~N}$ & 69 & 96 & 127 & 151 & 182 & 256 \\
\hline 13 & T13SW & 67 & 101 & 133 & 154 & 173 & 201 \\
\hline 13 & T13NW & 67 & 93 & 121 & 142 & 155 & 161 \\
\hline 151 & T151E & 30 & 48 & 80 & 107 & 127 & 143 \\
\hline 151 & T151SE & 40 & 56 & 95 & & & \\
\hline 151 & T151S & 34 & 81 & 128 & 221 & & \\
\hline 151 & T151SW & 30 & 37 & 51 & 83 & 111 & 130 \\
\hline 151 & T151NE & 34 & 49 & 59 & 66 & 84 & 130 \\
\hline 151 & T151NW & 30 & 41 & 65 & 91 & 106 & 115 \\
\hline 170 & T170NW & 14 & 18 & 25 & 34 & 48 & 78 \\
\hline 170 & T170W & 46 & & & & & \\
\hline 151 & T151W & 34 & 47 & 92 & 143 & 169 & 202 \\
\hline
\end{tabular}


Table C.3. Penetration resistance ( 10 to $15 \mathrm{ft}$ ) of SWSA 6 burial trenches and control sites used for leachate monitoring wells

\begin{tabular}{|c|c|c|c|c|c|c|c|}
\hline \multirow{2}{*}{$\begin{array}{l}\text { Trench } \\
\text { No. }\end{array}$} & \multirow{2}{*}{$\begin{array}{l}\text { Well } \\
\text { No. }\end{array}$} & \multicolumn{6}{|c|}{ Cumulaticve blow counts } \\
\hline & & $10 \mathrm{ft}$ & $11 \mathrm{ft}$ & $12 \mathrm{ft}$ & $13 \mathrm{ft}$ & $14 \mathrm{ft}$ & $16 \mathrm{ft}$ \\
\hline 103 & T103B & 60 & 62 & 65 & 69 & 71 & \\
\hline 103 & T103A & 56 & 60 & 62 & 65 & 68 & 72 \\
\hline 380 & None & 60 & 67 & 75 & & & \\
\hline 380 & T380 & 33 & 34 & 36 & & & \\
\hline 370 & T370 & 123 & 130 & 143 & 209 & & \\
\hline 370 & None & 111 & 124 & & & & \\
\hline 370 & None & 114 & 128 & 163 & & & \\
\hline 82 & T82 & 48 & 50 & 51 & 53 & 55 & 63 \\
\hline 120 & T120 & 59 & 62 & 65 & 71 & 73 & \\
\hline 408 & None & 28 & 30 & 39 & & & \\
\hline 408 & None & 31 & 33 & & & & \\
\hline 408 & T408 & 26 & 29 & 35 & 39 & 66 & 75 \\
\hline 397 & T397 & 65 & 70 & 71 & 80 & 99 & 121 \\
\hline 330 & T330 & 71 & 74 & 77 & 79 & 89 & 96 \\
\hline 363 & None & 64 & 67 & & & & \\
\hline 363 & None & & & & & & \\
\hline 363 & None & 61 & 68 & & & & \\
\hline 363 & T363 & 53 & 60 & 79 & 116 & & \\
\hline 69 & T69 & 125 & 163 & 188 & 221 & 265 & 308 \\
\hline 71 & None & & & & & & \\
\hline 71 & None & & & & & & \\
\hline 71 & None & & & & & & \\
\hline 71 & None & & & & & & \\
\hline 71 & None & & & & & & \\
\hline 71 & None & 66 & 95 & 123 & & & \\
\hline 121 & T121 & 54 & 57 & 61 & 64 & 68 & 74 \\
\hline 84 & None & 80 & 85 & & & & \\
\hline 84 & T84 & 67 & 68 & 70 & 72 & 74 & \\
\hline 308 & T308 & 126 & 194 & & & & \\
\hline 308 & None & & & & & & \\
\hline 318 & T318 & 128 & & & & & \\
\hline 112 & T112 & 81 & 117 & 156 & & & \\
\hline 85 & T85 & 96 & 131 & & & & \\
\hline 110 & T110 & 100 & & & & & \\
\hline 105 & T105 & 91 & 109 & & & & \\
\hline 426 & T426 & 76 & 123 & & & & \\
\hline 432 & T432 & 37 & 45 & 89 & & & \\
\hline 414 & T414 & 97 & & & & & \\
\hline 413 & T413 & 69 & & & & & \\
\hline 395 & T395 & 124 & & & & & \\
\hline 329 & T329 & 61 & & & & & \\
\hline 79 & None & & & & & & \\
\hline 79 & T79 & & & & & & \\
\hline 101 & T101 & 114 & 143 & & & & \\
\hline 461 & T461 & 123 & & & & & \\
\hline 424 & None & & & & & & \\
\hline 424 & T424 & 75 & 94 & 111 & 133 & 171 & \\
\hline & & & $C-8$ & & & & \\
\hline
\end{tabular}


Table C. 3 (continued)

\begin{tabular}{|c|c|c|c|c|c|c|c|}
\hline \multirow{2}{*}{$\begin{array}{l}\text { Trench } \\
\text { No. }\end{array}$} & \multirow{2}{*}{$\begin{array}{l}\text { Well } \\
\text { No. }\end{array}$} & \multicolumn{5}{|c|}{ Cumulaticve blow counts } & \multirow[b]{2}{*}{$16 \mathrm{ft}$} \\
\hline & & $10 \mathrm{ft}$ & $11 \mathrm{ft}$ & $12 \mathrm{ft}$ & $13 \mathrm{ft}$ & $14 \mathrm{ft}$ & \\
\hline 373 & None & 60 & 68 & 87 & 110 & 124 & 162 \\
\hline 373 & T373 & 65 & 70 & 81 & 98 & 123 & 139 \\
\hline 352 & T352 & 40 & 45 & 59 & 67 & 84 & 110 \\
\hline 367 & T367 & 72 & 95 & 124 & & & \\
\hline 453 & T453 & 96 & & & & & \\
\hline 422 & T422 & 132 & & & & & \\
\hline 374 & T374 & 53 & 66 & 75 & 109 & & \\
\hline 241 & $\mathrm{~T} 241$ & 47 & 51 & 70 & & & \\
\hline 237 & T237 & 34 & 35 & 39 & 43 & 54 & 66 \\
\hline 180 & $\mathrm{~T} 180$ & 49 & 52 & 58 & 70 & 80 & 112 \\
\hline 162 & T162 & 43 & 49 & 64 & & & \\
\hline 60 & T60 & 48 & 72 & 113 & & & \\
\hline 57 & None & & & & & & \\
\hline 57 & T57 & 65 & 69 & 73 & 77 & 79 & 86 \\
\hline 44 & T44 & 31 & 33 & 34 & 36 & 36 & 37 \\
\hline 63 & $\mathrm{~T} 63$ & 46 & 47 & 50 & 59 & 71 & 83 \\
\hline 225 & T225 & 44 & 47 & 51 & 62 & 82 & 92 \\
\hline 279 & T279-6 & 58 & 71 & 98 & 139 & 207 & 231 \\
\hline 288 & T288-6 & 41 & 76 & 144 & & & \\
\hline 13 & T13-6 & 64 & 109 & & & & \\
\hline 279 & T279-1 & 71 & 88 & & & & \\
\hline 279 & T279-2 & 61 & & & & & \\
\hline 279 & T279-3 & & & & & & \\
\hline 279 & $\mathrm{~T} 279-4$ & 42 & 84 & & & & \\
\hline 279 & T279-5 & 75 & 103 & 126 & 219 & 289 & 312 \\
\hline 288 & T288-1 & 35 & 38 & 58 & 91 & 138 & 188 \\
\hline 288 & $\mathrm{~T} 288-2$ & 28 & 67 & 90 & 95 & 115 & 168 \\
\hline 288 & T288-3 & 34 & 74 & & & & \\
\hline 288 & T288-4 & 44 & 74 & 107 & 130 & 155 & 218 \\
\hline 288 & T288-5 & 130 & & & & & \\
\hline 288 & T288N & 81 & 92 & 122 & 159 & 183 & 218 \\
\hline 13 & T13-5 & 33 & 88 & 170 & & & \\
\hline 13 & T13-4 & 22 & 29 & & & & \\
\hline 13 & $\mathrm{~T} 13-3$ & 29 & 63 & 148 & & & \\
\hline 13 & T13-2 & 40 & 69 & & & & \\
\hline 13 & T13-1 & 60 & 113 & 201 & & & \\
\hline 11 & T11 & 59 & 71 & 110 & & & \\
\hline 16 & T16 & 56 & & & & & \\
\hline 252 & T252 & 45 & 93 & 115 & 135 & & \\
\hline 285 & T285 & 133 & 160 & 184 & 248 & & \\
\hline 284 & T284 & 116 & & & & & \\
\hline 275 & $\mathrm{~T} 275$ & 110 & 148 & 160 & 191 & & \\
\hline
\end{tabular}


Table C. 3 (continued)

\begin{tabular}{|c|c|c|c|c|c|c|c|}
\hline \multirow{2}{*}{$\begin{array}{l}\text { Trench } \\
\text { No. }\end{array}$} & \multirow{2}{*}{$\begin{array}{l}\text { We11 } \\
\text { No. }\end{array}$} & \multicolumn{6}{|c|}{ Cumulaticve blow counts } \\
\hline & & $10 \mathrm{ft}$ & $11 \mathrm{ft}$ & $12 \mathrm{ft}$ & $13 \mathrm{ft}$ & $14 \mathrm{ft}$ & $16 \mathrm{ft}$ \\
\hline \multicolumn{8}{|c|}{ Wells near but outside burial trenches } \\
\hline 279 & T279N & 309 & & & & & \\
\hline 279 & T279S & 382 & 413 & 476 & & & \\
\hline 279 & T279NE & & & & & & \\
\hline 279 & T279SE & 333 & 403 & & & & \\
\hline 279 & T279NW & 287 & & & & & \\
\hline 279 & T279SW & 308 & & & & & \\
\hline 288 & T288S & 425 & 530 & 616 & 666 & & \\
\hline 288 & T288NW & 266 & 293 & 297 & 317 & 353 & \\
\hline 288 & $\mathrm{~T} 288 \mathrm{SW}$ & 258 & 285 & 297 & 324 & 378 & \\
\hline 288 & T288NE & 241 & 286 & & & & \\
\hline 288 & T288SE & 252 & 283 & 298 & 306 & 325 & 406 \\
\hline 13 & T13SE & 311 & 352 & 421 & 508 & & \\
\hline 13 & T13NE & 278 & 308 & & & & \\
\hline 13 & T13S & 328 & 369 & 414 & 474 & & \\
\hline 13 & $\mathrm{~T} 13 \mathrm{~N}$ & 320 & & & & & \\
\hline 13 & T13SW & 226 & 250 & 272 & & & \\
\hline 13 & T13NW & 170 & 202 & 264 & 336 & & \\
\hline 151 & T151E & 163 & 190 & 251 & & & \\
\hline 151 & T151SE & & & & & & \\
\hline 151 & T151S & & & & & & \\
\hline 151 & T151SW & 142 & 154 & 164 & 175 & 187 & 202 \\
\hline 151 & T151NE & 174 & 202 & 265 & & & \\
\hline 151 & T151NW & 127 & 146 & 163 & 215 & & \\
\hline 170 & T170NW & 123 & 174 & 222 & 274 & 326 & 372 \\
\hline 170 & T170W & & & & & & \\
\hline 151 & $\mathrm{~T} 151 \mathrm{~W}$ & & & & & & \\
\hline
\end{tabular}


APPENDIX D: Chemical Analyses of SWSA 6 Burial Trench Leachates 
Thable D.1. Chemical analyses of SUSA 6 burial trench leachates

\begin{tabular}{|c|c|c|c|c|c|c|c|}
\hline $\begin{array}{l}\text { Sampling Date: } \\
\text { Burial Trench Nuber: } \\
\text { Species/Chemical: }\end{array}$ & CAS NO. & $\begin{array}{l}26 \mathrm{Feb} 88 \\
\mathrm{~T}-5 \\
\text { (pob) } \\
\end{array}$ & $\begin{array}{l}21 \ln 89 \\
\mathrm{~T}-4 \\
\text { (ppb) }\end{array}$ & $\begin{array}{l}\text { 21Jun89 } \\
\mathrm{T}-7 \\
\text { (pdb) } \\
\end{array}$ & $\begin{array}{c}11 \text { Augg9 } \\
\mathrm{T}-13 \\
\text { (ppb) } \\
\end{array}$ & $\begin{array}{c}11 \text { Augg9 } \\
\text { T-279 } \\
\text { (ppb) }\end{array}$ & $\begin{array}{c}11 \text { Auggs } \\
\text { T-288 } \\
\text { (ppb) }\end{array}$ \\
\hline Chlonamethane & $74-87-3$ & $\mathrm{NA}$ & $\triangleleft 0.00$ & 40.00 & $\triangle 10.00$ & 40.00 & $\triangle 0.00$ \\
\hline Bromomethane & $74-83-9$ & NA & $\triangleleft 0.00$ & $<10.00$ & $\triangleleft 0 . \infty$ & $\triangleleft 10.00$ & $\triangleleft 0.00$ \\
\hline Vinyl Chloride & $75-01-4$ & NA & $\triangleleft 0.00$ & $\varangle 0 . \infty$ & $<10.00$ & $<10 . \infty$ & $<0.00$ \\
\hline Chlonoethane & $75-00-3$ & NA & $\triangleleft 0 . \infty 0$ & $\triangleleft 0 . \infty$ & $\triangleleft 0.00$ & $<0.00$ & $<0.00$ \\
\hline Methylene chloride & $75-09-2$ & NA & $\triangleleft 0 . \infty$ & $B 450.00$ & B50.00 & B7. 00 & $\mathrm{JB} 3.00$ \\
\hline Acetane & $67-64-1$ & NA & $\mathrm{B} 77 . \infty$ & JB6. 00 & B140.00 & $<10.00$ & $\mathrm{JB} 4 . \infty$ \\
\hline Carban disulfide & $75-15-0$ & $\mathrm{NA}$ & 5.00 & $<. \infty$ & $\diamond .00$ & 6.00 & $\diamond .00$ \\
\hline 1,1-Dichloroethene & $75-35-4$ & NA & $<.00$ & $\diamond . \infty$ & $\diamond .00$ & $\diamond .00$ & $\diamond .00$ \\
\hline 1,1-Dichlonoethane & $75-34-3$ & NA & 5.00 & $\diamond .00$ & $<.00$ & $\$ .00$ & $<.00$ \\
\hline 1,2-Dichloroethene (Total) & $540-59-0$ & $\mathrm{NA}$ & $<.00$ & $\diamond . \infty$ & $\diamond .00$ & 5.00 & $<.00$ \\
\hline Chloroform & $67-66-3$ & NA & $\diamond .00$ & $\delta . \infty$ & 35.00 & $\mathrm{~J} 5.00$ & $<.00$ \\
\hline 1,2-Dichloroethane & $107-06-2$ & $\mathrm{NA}$ & $\varsigma .00$ & $\diamond . \infty$ & $\diamond .00$ & $\mathrm{~J} 3.00$ & $<.00$ \\
\hline 2-Butanane & $78-93-3$ & NA & $\triangleleft 0.00$ & 40.00 & $<0.00$ & $40 . \infty$ & $\triangleleft 0.00$ \\
\hline 1,1,1-Trichloncethane & $71-55-6$ & $\mathrm{NA}$ & $\diamond .00$ & $\diamond . \infty$ & $<.00$ & $\diamond .00$ & $<.00$ \\
\hline Carbon tetrachloride & $56-23-5$ & NA & $\varsigma .00$ & $\diamond . \infty$ & $\diamond .00$ & $\diamond . \infty$ & $<.00$ \\
\hline Vinyl acetate & $108-05-4$ & NA & $<0 . \infty$ & $<10.00$ & $\triangleleft 10.00$ & $\triangleleft 0.00$ & 40.00 \\
\hline Bramotichlaramethane & $75-27-4$ & NA & $<.00$ & $\diamond . \infty$ & $<.00$ & $<.00$ & $<.00$ \\
\hline $1,2-I$ & $78-87-5$ & NA & $\diamond .00$ & $\diamond . \infty$ & $\diamond .00$ & $\varsigma .0$ & $<.00$ \\
\hline cis-1,3-Dichloropropene & $10061-01-5$ & NA & 5.00 & $\diamond . \infty$ & 5.00 & $<5.00$ & $<.00$ \\
\hline Trichloro & $79-01-6$ & NA & 5.00 & $\diamond . \infty$ & $\diamond .00$ & $\diamond .00$ & $<.00$ \\
\hline amethane & $124-48-1$ & $\mathrm{NA}$ & 5.00 & $\diamond . \infty$ & $\$ .00$ & 5.00 & $<.00$ \\
\hline 1,1,2-Trichlonoethane & $79-\infty 0-5$ & NA & 5.00 & $\diamond .00$ & 5.00 & $\varsigma .00$ & $<.00$ \\
\hline Benzene & $7 \pi-43-2$ & NA & $\diamond .00$ & $\diamond . \infty$ & 240.00 & 140.00 & $\mathrm{~J} 2.00$ \\
\hline trans-1,3-Dichloropropene & $10061-02-06$ & NA & $\diamond .00$ & $\diamond .00$ & $\diamond .00$ & $\varangle .00$ & $<.00$ \\
\hline Bramofarm & $75-25-2$ & NA & $\diamond .00$ & 5.00 & $\diamond .00$ & $\diamond .00$ & $<.00$ \\
\hline 4-Methyl-2-pentamane & 108 & NA & $\triangleleft 0.00$ & $\triangleleft 0.00$ & $<10.00$ & $\triangleleft 0.00$ & $<0.00$ \\
\hline 2-Hexamane & $591-$ & NA & $\triangleleft 10.00$ & 40.00 & $\varangle 0.00$ & $\triangleleft 0.00$ & $<10.00$ \\
\hline Tetrachloroethene & $127-18-4$ & $\mathrm{NA}$ & $\varangle .00$ & $<. \infty$ & $<.00$ & $\diamond .00$ & $<.00$ \\
\hline 1,1,2,2-tetrachlancethane & $79-34-5$ & NA & $<.00$ & 5.00 & $\varsigma .00$ & $\checkmark .00$ & $<.00$ \\
\hline Toluene & 108 & NA & $<.00$ & $<.00$ & 4300.00 & 3400.00 & 180.00 \\
\hline Chloroberzene & $108-90-7$ & NA & $<.00$ & 5.00 & $<.00$ & $<.00$ & $<.00$ \\
\hline Ethylberzene & 100 & $N A$ & $\delta . \infty$ & $\diamond . \infty$ & 6000.00 & 280.00 & $<.00$ \\
\hline Styrene & 100 & NA & $<.00$ & $<.00$ & $\diamond .00$ & 5.00 & $<.00$ \\
\hline Xylene (Total) & $1330-20-7$ & MA & $<.00$ & 5.00 & 32000.00 & 820.00 & 480.00 \\
\hline Fenol & $108-95-2$ & NA & $<1.00$ & 41.00 & 690.00 & 38.00 & $J 2.00$ \\
\hline bis(2-Chlonoethyl)ether & $111-44-4$ & NA & $\triangleleft 11.00$ & $\triangleleft 1.00$ & $\varangle 3.00$ & $\varangle 4.00$ & $\varangle 3.00$ \\
\hline 2-Chlorophenol & $95-57-8$ & $\mathrm{NA}$ & $<1.00$ & 41.00 & $<3.00$ & $\varangle 4.00$ & $<3.00$ \\
\hline moberzene & $541-73-1$ & NA & $\ll 1.00$ & $\triangleleft 1 . \infty$ & $\varangle 3.00$ & $\varangle 4.00$ & 43.00 \\
\hline ondberzene & $106-46-7$ & NA & $\triangleleft 11.00$ & $\triangleleft 1 . \infty$ & 43.00 & $\varangle 4.00$ & $\triangleleft 3.00$ \\
\hline Berzyl alochol & $100-51-6$ & NA & $\triangleleft 11.00$ & $\triangleleft 11.00$ & $\triangleleft 3.00$ & $\varangle 4.00$ & $<3.00$ \\
\hline 1,2-Dichloroberzene & $95-50-1$ & $\mathbb{N A}$ & $\triangleleft 11.00$ & 41.00 & $<3.00$ & $\varangle 4.00$ & $\varangle 3.00$ \\
\hline 2-Methylphenol & $95-48-7$ & NA & $\triangleleft 1.00$ & $\triangle 1.00$ & 46.00 & 64.00 & 16.00 \\
\hline bis (2-Chlonoisopropyl)ether & $108-60-1$ & NA & $\triangleleft 1.00$ & $\triangleleft 1 . \infty 0$ & $<3.00$ & $\varangle 4.00$ & $\varangle 3.00$ \\
\hline 4-Methylphenol & $106-44-5$ & NA & $\triangleleft 1.00$ & 41.00 & 3900.00 & 120.00 & 25.00 \\
\hline N-nitroso-di-n- & $621-64-7$ & $\mathrm{NA}$ & $\triangleleft 1.00$ & $\triangleleft 1.00$ & $\triangleleft 3.00$ & 44.00 & 43.00 \\
\hline
\end{tabular}


Table D.1 (continued)

\begin{tabular}{|c|c|c|c|c|c|c|c|}
\hline $\begin{array}{l}\text { Sampling Date: } \\
\text { Burial Trench Nunber: } \\
\text { Species/Chemical: }\end{array}$ & CAS Nb. & $\begin{array}{c}26 \text { Feb88 } \\
\text { T-5 } \\
\text { (ppb) } \\
\end{array}$ & $\begin{array}{l}217 \text { ro9 } \\
T-4 \\
\text { (ppb) }\end{array}$ & $\begin{array}{l}21 \text { lin89 } \\
T-7 \\
\text { (pab) }\end{array}$ & $\begin{array}{l}11 \text { Augf9 } \\
\text { T-13 } \\
\text { (ppb) }\end{array}$ & $\begin{array}{c}11 \text { Augg9 } \\
\text { T-279 } \\
\text { (ppb) }\end{array}$ & $\begin{array}{l}11 \text { Augg9 } \\
T-288 \\
\text { (ppb) }\end{array}$ \\
\hline Hexachloroethane & $67-72-1$ & NA & 41.00 & 41.00 & $<13.00$ & 44.00 & 43.00 \\
\hline Nitrobenzene & $98-95-3$ & NA & 41.00 & 41.00 & $\triangleleft 3.00$ & $<14.00$ & 43.00 \\
\hline Isopharane & $78-59-1$ & $\mathrm{M}$ & 41.00 & 41.00 & $\varangle 3.00$ & $\varangle 4.00$ & $\varangle 3.00$ \\
\hline 2-Nitropheml & $88-75-5$ & NA & 41.00 & 41.00 & $\triangleleft 3.00$ & $<4.00$ & $\triangleleft 3.00$ \\
\hline 2,4-Dimethylphenol & $105-67-9$ & NA & $\triangleleft 11.00$ & $<11.00$ & 260.00 & 27.00 & 17.00 \\
\hline Berzoic acid & 6 & NA & 56.00 & 56.00 & 300.00 & 110.00 & Л 0.00 \\
\hline bis(2-chlonoethaxy)methane & $111-91-1$ & NA & 41.00 & $\triangleleft 1.00$ & 43.00 & $\triangleleft 4.00$ & $\varangle 3.00$ \\
\hline 2,4-Dichla & $120-83-2$ & NA & 41.00 & 41.00 & .00 & $<4.00$ & $\varangle 3.00$ \\
\hline benzene & $120-82-1$ & NA & $\triangleleft 11.00$ & 41.00 & $\triangleleft 3.00$ & $\triangleleft 4.00$ & $\triangleleft 3.00$ \\
\hline alene & $1-20-3$ & NA & 41.00 & 41.00 & 4000.00 & $<14.00$ & 57.00 \\
\hline $4-d$ & 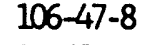 & NA & $\triangleleft 1.00$ & $41 . \infty 0$ & $\triangleleft 3.00$ & $\triangleleft 4.00$ & $<3.00$ \\
\hline thene & 87 & NA & $\triangleleft 1.00$ & $\triangleleft 1.00$ & $\varangle 3.00$ & $\varangle 4.00$ & $\varangle 3.00$ \\
\hline ylphenol & $59-50-7$ & NA & $\triangleleft 11.00$ & $\triangleleft 11.00$ & $<3.00$ & .00 & $<3.00$ \\
\hline $2-M$ & $91-57-6$ & NA & $\triangleleft 1.00$ & 41.00 & $\varangle 3.00$ & $\triangleleft 4.00$ & 43.00 \\
\hline Hex & $77-47-4$ & NA & $\varangle 1.00$ & $\triangleleft 11.00$ & 43.00 & $<4.00$ & 43.00 \\
\hline & 2 & NA & $\triangleleft 11.00$ & $\triangleleft 1.00$ & $\varangle 3.00$ & $\triangleleft 4.00$ & 43.00 \\
\hline 2, & 4 & NA & $<6.00$ & $<6.00$ & 86.00 & $<1.00$ & 67.00 \\
\hline $2-a$ & $91-58-7$ & NA & $\triangleleft 11.00$ & 41.00 & $<3.00$ & $\infty$ & 43.00 \\
\hline $2-N 6$ & $88-74-4$ & NA & 56.00 & $<6.00$ & 67.00 & $<1 . \infty$ & 67.00 \\
\hline Dims & $31-11-3$ & NA & 41.00 & 41.00 & $\varangle 3.00$ & 44.00 & 43.00 \\
\hline & 2 & NA & $<1.00$ & 41.00 & $\varangle 3.00$ & $\triangle 4.00$ & 43.00 \\
\hline $\mathbf{e}$ & $606-20-2$ & A & $\varangle 1.00$ & $41 . \infty$ & $<3.00$ & $<4.00$ & 43.00 \\
\hline $3-\mathrm{N}$ & $99-c$ & $\mathrm{NA}$ & 56.00 & $<6.00$ & $\infty$ & & 67.00 \\
\hline $\mathrm{Aos}$ & $83-3$ & NA & $\triangleleft 11.00$ & 41.00 & $\triangleleft 3.00$ & $<4.00$ & 43.00 \\
\hline enol & $51-28-5$ & $\mathrm{NA}$ & 56.00 & 56.00 & 67.00 & $<1 . \infty$ & $\varangle 7.00$ \\
\hline & 100 & NA & 56.00 & $\diamond 6.00$ & $\varangle 67.00$ & $<1 . \infty$ & 67.00 \\
\hline & 132 & NA & $\triangleleft 11.00$ & $\triangleleft 11.00$ & $\varangle 3.00$ & 44.00 & $\triangleleft 3.00$ \\
\hline & & A & $\varangle 1.00$ & $41 . \infty$ & $\triangleleft 3.00$ & 44.00 & $\varangle 13.00$ \\
\hline bie & $84-1$ & NA & $\triangleleft 11.00$ & & & & 13.00 \\
\hline 4-a & $7005-72-3$ & NA & $\triangleleft 11.00$ & $\varangle 1 . \infty$ & 43.00 & 44.00 & 43.00 \\
\hline Fle & $86-7$ & NA & $\triangleleft 11.00$ & 41.00 & 43.00 & 44.00 & 43.00 \\
\hline$-\mathrm{N}^{-}$ & $100-c$ & NA & $\$ 6.00$ & $\$ 6.00$ & $<67.00$ & $\langle 1 . \infty$ & 67.00 \\
\hline 4,6 & 534 & $\mathrm{NA}$ & 56.00 & $<6.00$ & 87.00 & $<1.00$ & 87.00 \\
\hline & & NA & $\infty$ & $\infty$ & & $<4.00$ & $<3.00$ \\
\hline $4-\mathrm{b}$ & 101 & NA & $\triangleleft 11.00$ & $41 . \infty$ & $<3.00$ & & 3.0 \\
\hline Hexe & $118-$ & NA & $\triangleleft 11.00$ & $\triangleleft 1.00$ & $<3.00$ & 44.00 & $<3.00$ \\
\hline henol & $87-8$ & NA & 56.00 & $<6.00$ & $<67.00$ & $\langle 1.00$ & 867.00 \\
\hline mene & $85-0$ & NA & $<1.00$ & 41.00 & $<3.00$ & $<4.00$ & $\varangle 3.00$ \\
\hline & 120 & NA & $<1.00$ & $\triangleleft 1 . \infty$ & $<3.00$ & $<4.00$ & $<3.00$ \\
\hline & & NA & JB1 & $\infty$ & JB2.00 & JB2.00 & $\mathrm{JB} 2.00$ \\
\hline Whe & 2 & $\mathrm{NA}$ & 41.00 & 41.00 & 43.00 & $<14.00$ & $<13.00$ \\
\hline Pyre & $129-\infty$ & & $<1.00$ & 41.00 & 43.00 & $\triangleleft 4.00$ & 43.00 \\
\hline Butylbere & $85-68-7$ & $\mathrm{NA}$ & $\triangleleft 1.00$ & $\triangleleft 1 . \infty$ & 43.00 & $<4.00$ & 43.00 \\
\hline 3,3'-Dichlo & $91-94-1$ & NA & 22.00 & $<2.00$ & $<27.00$ & $<9.00$ & $<7.00$ \\
\hline Berzo(a)a & $56-55-3$ & NA & $\varangle 1.00$ & $\triangleleft 1 . \infty$ & $\triangle 3.00$ & 44.00 & $\varangle 3.00$ \\
\hline Anryser & 218-01-9 & NA & $\triangleleft 11.00$ & $\triangleleft 11.00$ & $\triangle 3.00$ & $<14.00$ & $<3.00$ \\
\hline
\end{tabular}


Table D.1 (continued)

\begin{tabular}{|c|c|c|c|c|c|c|c|}
\hline $\begin{array}{l}\text { Sampling Date: } \\
\text { Burial Trench Number: } \\
\text { Species/Chemical: }\end{array}$ & CAS No. & $\begin{array}{l}\text { 26Feb88 } \\
\mathrm{T}-5 \\
\text { (ppb) } \\
\end{array}$ & $\begin{array}{l}21 \mathrm{ur} 89 \\
\mathrm{~T}-4 \\
\text { (ppb) }\end{array}$ & $\begin{array}{l}21 \mathrm{run} 89 \\
\mathrm{~T}-7 \\
\text { (ppb) }\end{array}$ & $\begin{array}{l}\text { 11/ugge } \\
\mathrm{T}-13 \\
\text { (ppb) }\end{array}$ & $\begin{array}{c}\text { 11Augg9 } \\
\text { T-279 } \\
\text { (ppb) }\end{array}$ & $\begin{array}{c}11 \text { Augh9 } \\
\text { T-288 } \\
\text { (pb) }\end{array}$ \\
\hline bis(2-Ethylhexyl)phalate & $\overline{117-81-7}$ & $\mathrm{NA}$ & $\triangle 1.00$ & 41.00 & 43.00 & $<14.00$ & $\overline{43.00}$ \\
\hline Di.-n-Octylphthalate & $117-84-0$ & NA & $\triangleleft 1.00$ & 41.00 & $<3.00$ & $<14.00$ & $<3.00$ \\
\hline Benzo(b) flumpanthene & $205-99-2$ & NA & $\triangleleft 1.00$ & 41.00 & $<3.00$ & $<4.00$ & $\varangle 3.00$ \\
\hline Berzo(k) fluoranthere & $207-08-9$ & NA & $\triangleleft 11.00$ & 41.00 & $<3.00$ & $\triangleleft 4.00$ & $\varangle 3.00$ \\
\hline Benzo(a)pyrene & $50-32-8$ & NA & $<11.00$ & $\triangleleft 1.00$ & $<3.00$ & $<14.00$ & $\varangle 3.00$ \\
\hline Indeno $(1,2,3-\infty d)$ pyrene & $193-39-5$ & $\mathrm{NA}$ & $\triangleleft 1.00$ & $\triangleleft 1.00$ & 43.00 & $<4.00$ & $\triangleleft 3.00$ \\
\hline Diberz $(a, h)$ anthracene & $53-70-3$ & NA & $\triangle 1.00$ & 41.00 & $<3.00$ & $<4.00$ & $\triangleleft 3.00$ \\
\hline $\operatorname{Benzo}(\mathrm{g}, \mathrm{h}, \mathrm{i})$ perylene & $191-24-2$ & NA. & $\triangleleft 1.00$ & $\triangleleft 1 . \infty$ & $\varangle 3.00$ & $\varangle 4.00$ & $\triangleleft 3.00$ \\
\hline Acrylamide & $78-06-1$ & $\mathrm{NA}$ & NA & NA & NA & NA & NA \\
\hline Silver & $\mathrm{Ag}$ & $\mathbf{N}$ & 6 & 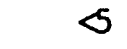 & 7 & $\diamond$ & $<$ \\
\hline Aluminm & $\mathrm{Al}$ & NA & 510 & 510 & 210 & $<0$ & $\measuredangle 0$ \\
\hline Arsenic & As & NA & 60 & 87 & $<0$ & $<0$ & 50 \\
\hline Bonan & B & $\mathrm{NA}$ & $<8$ & $<80$ & $<8$ & $<80$ & $<0$ \\
\hline Barium & $\mathrm{Ba}$ & NA & 120 & 170 & 440 & 550 & 430 \\
\hline Beryllium & $\mathrm{Be}$ & NA & $\diamond .4$ & $\varangle .4$ & $\varangle .4$ & $\diamond .4$ & $\varangle .4$ \\
\hline Calcium & $\mathrm{Ca}$ & NA & 40000 & 99000 & 110000 & 24000 & 58000 \\
\hline Cadmin & $\mathrm{Cd}$ & $\mathrm{NA}$ & 290 & $<$ & $<$ & $<$ & $<$ \\
\hline Cobalt & $C_{0}$ & NA & 30 & $<$ & 7 & 3 & 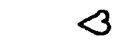 \\
\hline Chramium & $a r$ & $\mathrm{NA}$ & 14 & 20 & 43 & 36 & 27 \\
\hline Copper & $a_{1}$ & NA & 13 & 40 & 29 & 27 & 14 \\
\hline Iran & $\mathrm{Fe}$ & NA & 250 & 2 & 10 & $\triangleleft 0$ & $\triangleleft 0$ \\
\hline Merary & Hg & NA & 5 & $\varangle .1$ & 1.0 & $\varangle .1$ & $\varangle .1$ \\
\hline Potassium & $\mathrm{k}$ & NA & $N A$ & NA & 7200 & 3900 & 1800 \\
\hline Lithium & Li & NA & $<15000$ & $\varangle 5000$ & $\varangle 5000$ & 45000 & $\varangle 5000$ \\
\hline Magnesium & Mg & NA & 700 & 17000 & 28000 & 27000 & 17000 \\
\hline Manganese & Mn & $\mathrm{NA}$ & 3100 & 11 & 2700 & 27 & 440 \\
\hline Molybdenum & Mo & NA & 40 & $\$ 0$ & 40 & $\$ 0$ & 40 \\
\hline Sodium & $\mathrm{Na}$ & $N A$ & 6800 & 28000 & 13000 & 74,00 & $<2000$ \\
\hline Nickel & $\mathrm{Ni}$ & NA & 190 & $\varangle$ & 46 & 6 & 6 \\
\hline Prosphons & $\mathrm{P}$ & NA & $\infty 00$ & $<00$ & $\infty \infty 0$ & $<00$ & $<00$ \\
\hline Lead & BD & NA & $\infty$ & $\infty$ & $<0$ & $<0$ & $<0$ \\
\hline Antimany & Sb & $\mathrm{NA}$ & 40 & $\varangle 0$ & $\varangle 0$ & $\$ 0$ & 40 \\
\hline Selenium & Se & NA & $<8$ & $<80$ & $<80$ & $<80$ & $<80$ \\
\hline Silioan & Si & $\mathrm{NA}$ & 2600 & 3400 & 800 & 5200 & 3800 \\
\hline $\operatorname{Tin}$ & Sn & $\mathrm{NA}$ & $<0$ & $<0$ & $<0$ & $<0$ & $<0$ \\
\hline Stratium & $\mathrm{Sr}$ & NA & 75 & 140 & 210 & 160 & 130 \\
\hline Titanium & Ti & $\mathrm{NA}$ & $<20$ & $\infty$ & $\infty 0$ & $\infty$ & $<0$ \\
\hline Vanadium & V & NA & 4 & 4 & 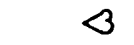 & $\infty$ & $\beta$ \\
\hline Zinc & $2 n$ & NA & 140 & $<8$ & 470 & 8 & 180 \\
\hline Ziroconium & $2 x$ & NA & $<0$ & $\infty$ & $<20$ & $\infty 0$ & $<20$ \\
\hline Bramide & $\mathrm{Br}-$ & NA & $\diamond 0$ & $<0$ & 80 & $<0$ & $<0$ \\
\hline Chloride & Cl- & NA & 4310 & 9100 & 47000 & 9300 & 1600 \\
\hline Fluoride & F- & $\mathrm{NA}$ & 500 & 830 & 6400 & 900 & 200 \\
\hline Nitrate & NO3- & NA & 750 & 3890 & 130 & 200 & 100 \\
\hline Phosphate & $\mathrm{PO}_{4}$ & NA & 50 & 120 & $<0$ & $<0$ & 50 \\
\hline Sulfate & $\mathrm{SO}_{4}$ & $\mathrm{NA}$ & 2440 & 56200 & 9100 & 8000 & 2200 \\
\hline
\end{tabular}


Table D.IL (continued)

\begin{tabular}{|c|c|c|c|c|c|c|c|}
\hline $\begin{array}{l}\text { Sampling Date: } \\
\text { Burial Trench Nuber: } \\
\text { Species/Chemical: }\end{array}$ & CAS NB, & $\begin{array}{c}26 \mathrm{Feb} 88 \\
\mathrm{~T}-5 \\
\text { (ppb) } \\
\end{array}$ & $\begin{array}{l}2 L \ln 89 \\
T-4 \\
-(p \mathrm{pb})\end{array}$ & $\begin{array}{c}21 \text { hrn89 } \\
\mathrm{T}-7 \\
\text { (ppb) }\end{array}$ & $\begin{array}{l}\text { 11Augg9 } \\
\mathrm{T}-13 \\
\text { (ppb) }\end{array}$ & $\begin{array}{l}11 \text { Augf9 } \\
\text { T-279 } \\
\text { (ppb) }\end{array}$ & $\begin{array}{c}11 \text { Augs9 } \\
\text { T-288 } \\
\text { (ppb) }\end{array}$ \\
\hline Total Organic Cauban & T.O.C. & $\mathrm{NA}$ & 6500 & 15200 & 166000 & 182000 & 41300 \\
\hline Canban-14 & $(\mathrm{Bg} / \mathrm{L})$ & NA & NA & NA & 38 & 19 & 9 \\
\hline Cobalt -60 & $(\mathrm{~Bq} / \mathrm{L})$ & NA & NA & NA & $\$ .1$ & $\varangle .1$ & $\varangle .1$ \\
\hline Cesium-137 & $(\mathrm{Bq} / \mathrm{L})$ & $\mathrm{NA}$ & NA & NA & $\$ .1$ & 36 & 1.5 \\
\hline Gross Alyha & $(B q / L)$ & 4500 & NA & NA & 0.55 & 1.0 & 0.56 \\
\hline Gross Beta & $(B q / L)$ & NA & NA & NA & 55 & 91 & 26 \\
\hline Strantium-90 & $(\mathrm{Bq} / \mathrm{L})$ & NA & NA & NA & 5.5 & 35 & 14 \\
\hline Tritium & $(B q / L)$ & NA & 2420 & $\mathrm{NA}$ & 11000 & 950 & 250 \\
\hline Americium-241 & $(B q / L)$ & 1.1 & NA & NA & NA & NA & NA \\
\hline arrium-244 & $(\mathrm{Bg} / \mathrm{L})$ & $\$ .1$ & NA & NA & NA & NA & NA \\
\hline Plutanium-239 & $(\mathrm{Bg} / \mathrm{L})$ & 0.8 & NA & NA & NA & NA & NA \\
\hline Uranium-232 & $(\mathrm{Bq} / \mathrm{L})$ & 58 & NA & NA & NA & NA & NA \\
\hline Uranium-233 & $(\mathrm{Bg} / \mathrm{L})$ & 3700 & NA & NA & NA & NA & NA \\
\hline $\mathrm{HH}$ & $-\log [H+]$ & NA & 7.7 & NA & 7.8 & 7.9 & 8 \\
\hline Electrical Conductivity & $(\mathrm{dS} / \mathrm{m})$ & NA & 194 & NA & 260 & 405 & 296 \\
\hline Dissolved Solids & $(\mathrm{mg} / \mathrm{L})$ & NA & 200 & 100 & 560 & 500 & 280 \\
\hline Total Solids & $(\mathrm{mg} / \mathrm{L})$ & NA & NA & NA & NA & NA & Na \\
\hline Suspended Solids & $(\mathrm{m} / \mathrm{L})$ & NA & NA & NA & NA & NA & NA \\
\hline Handhess & $(\mathrm{mg} / \mathrm{L})$ & NA & 122 & NA & 326 & 188 & 194 \\
\hline Alkalinity & $(\mathrm{mg} / \mathrm{L})$ & NA & 110 & NA & 420 & 160 & 240 \\
\hline
\end{tabular}


Thble D.2. Chemical analyses of SUSA 6 burial trench leachates

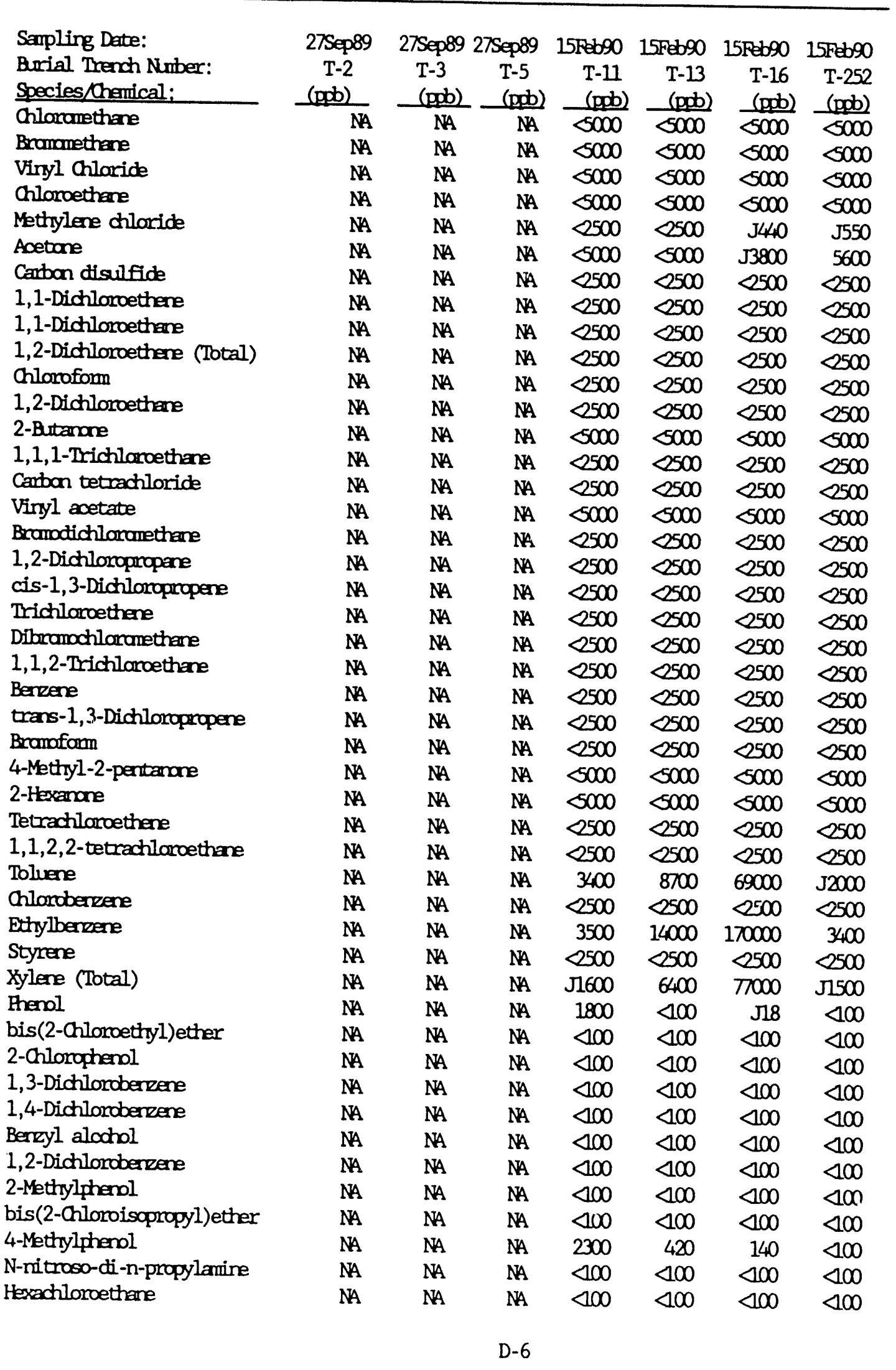


Table D.2 (continued)

\begin{tabular}{|c|c|c|c|c|c|c|c|}
\hline Sampling Date: & $275 e p 89$ & 27 Sep89 & 27Sep89 & $15 \mathrm{Feb} 90$ & 15Feb90 & $15 \mathrm{Feb} 90$ & $15 \mathrm{Feb} 90$ \\
\hline $\begin{array}{l}\text { Burial Trench Nuber: } \\
\text { Species/Chemical: }\end{array}$ & $\begin{array}{r}\mathrm{T}-2 \\
(\mathrm{ppb}) \\
\end{array}$ & $\begin{array}{l}T-3 \\
(p p b)\end{array}$ & $\begin{array}{l}\mathrm{T}-5 \\
\text { (ppb) }\end{array}$ & $\begin{array}{l}T-11 \\
(\mathrm{ppb})\end{array}$ & $\begin{array}{l}\mathrm{T}-13 \\
\text { (pob) } \\
\end{array}$ & $\begin{array}{l}\mathrm{T}-16 \\
\text { (ppb) }\end{array}$ & $\begin{array}{l}\mathrm{T}-252 \\
\text { (ppb) } \\
\end{array}$ \\
\hline Nitroberzene & $\mathrm{NA}$ & $\mathrm{NA}$ & NA & $<100$ & $\varangle 00$ & 410 & 100 \\
\hline Isophonane & NA & NA & NA & $<\infty$ & $<\infty$ & $\triangleleft 100$ & 400 \\
\hline 2-Nitrophenol & NA & NA & NA & $<00$ & 400 & $\Delta 00$ & $\triangle 00$ \\
\hline 2,4-Dimethylphenal & NA & NA & NA & $<100$ & 400 & 400 & 400 \\
\hline Berroic acid & NA & NA & NA & 2400 & $\$ 10$ & 570 & $\diamond 10$ \\
\hline bis(2-chloroethaxy) methane & NA & NA & NA & $\triangleleft 00$ & $<\infty$ & 400 & 400 \\
\hline 2,4-Dichlorophemi & ד̣a & NA & NA & 400 & 400 & $\Delta 00$ & 400 \\
\hline 1,2,4-Trichlimabenzene & NA & NA & NA & $<00$ & $\triangleleft \infty 0$ & $<100$ & 400 \\
\hline Nephthalene & NA & NA & NA & 1400 & 2300 & 400 & 160 \\
\hline 4-chloroaniline & NA & NA & NA & $\triangleleft \infty$ & 400 & $\triangle 00$ & 400 \\
\hline Hexachlonobutadiene & NA2 & NA & $\mathrm{NA}$ & $<100$ & $<00$ & $\triangleleft 00$ & 400 \\
\hline 4-Chloro-3-methylpherol & No. & NA & NA & $<00$ & $<100$ & $<10$ & $\triangle 100$ \\
\hline 2-Methylnephthalene & NA & NA & NA & $<00$ & $<100$ & 420 & $<100$ \\
\hline Hexachlorocyclopentadiene & M & NA & NA & 400 & 400 & $\triangle 100$ & 400 \\
\hline 2,4,6-Trichloropherol & NA & NA & NA & $\triangle 00$ & $\varangle 100$ & $4 \infty$ & $\triangle 00$ \\
\hline 2,4,5-Trichloropheml & No. & NA & $\mathrm{NA}$ & 510 & 510 & 520 & $<10$ \\
\hline 2-Chloranephthalere & NA & NA & NA & $\triangle \infty 0$ & $\triangle \infty 0$ & $\triangle 100$ & $<100$ \\
\hline 2-Nitroentiline & NA & NA & NA & $\diamond 10$ & 510 & $\checkmark 20$ & $\diamond 10$ \\
\hline Dimethylphthalate & NA & NA & NA & $<00$ & $<\infty$ & $\triangle 10$ & $\triangle \infty 0$ \\
\hline Acenaphuthylene & NA & NA & NA & $\triangleleft 00$ & 400 & $\triangle 100$ & $\triangle 00$ \\
\hline 2,6-Dinitratoluene & NA & NA & NA & $\triangleleft 00$ & $\triangleleft 00$ & $<100$ & $\triangle 100$ \\
\hline 3-Nitroaniline & NA & NA & NA & $\diamond 10$ & $<10$ & 520 & $\delta 10$ \\
\hline Acenaphthene & NA & NA & $\mathrm{NA}$ & $<100$ & $\triangle 100$ & $\Delta a)$ & $\triangle 100$ \\
\hline 2,4-Dinitrophenol & NA & NA & $\mathrm{NA}$ & $\checkmark 10$ & $<10$ & 520 & $\$ 10$ \\
\hline 4-Nitropherol & NA & NA & NA & $\diamond 10$ & $\$ 10$ & $<20$ & $\$ 10$ \\
\hline Dibenzofiran & NA & NA & $\mathrm{NA}$ & $<\infty$ & 400 & $<00$ & 400 \\
\hline 2,4-Dinitrotoluene & NA & NA & NA & $<100$ & 400 & $\triangleleft \infty 0$ & $4 \infty$ \\
\hline Diethylphthalate & NA & NA & NA & $\triangle 100$ & $\triangleleft \infty$ & $\triangle 00$ & $\triangle 00$ \\
\hline 4-Chlorophenyl-phenylether & NA & NA & NA & $<00$ & 400 & $<0$ & $<\infty$ \\
\hline Fluorene & NA & NA & $\mathrm{NA}$ & $<00$ & $<100$ & $<100$ & $<00$ \\
\hline 4-Nitroaniline & NA & $N A$ & NA & $<10$ & $\diamond 10$ & $<20$ & $\diamond 10$ \\
\hline 4,6-Dinitro-2-methylphenol & NA & NA & NA & $<10$ & $\checkmark 10$ & $<520$ & $\diamond 10$ \\
\hline N-nitrosodiphenylamine & NA & NA & NA & $\triangle 100$ & 400 & 400 & $\triangle \infty 0$ \\
\hline 4-bramophenyl-phanylether & NA & NA & $\mathrm{NA}$ & $<00$ & $\triangleleft, \infty$ & 400 & 400 \\
\hline Hexachlouicberzene & NA & NA & $\mathrm{NA}$ & $<100$ & $<100$ & $<100$ & $\triangle 00$ \\
\hline Fentachlorophenol & NA & NA & NA & $<10$ & $<10$ & $\$ 20$ & $\$ 10$ \\
\hline Fhenanthrene & NA & NA & NA & $\triangle 100$ & 400 & $\triangle 100$ & 400 \\
\hline Anthracene & NA & NA & NA & $<00$ & 400 & $<\infty$ & $<100$ \\
\hline Di-n-butylpinthalate & NA & NA & NA & $<\infty$ & $<\infty$ & $<100$ & $<\infty$ \\
\hline Fluocanthene & NA & NA & $\mathrm{NA}$ & $\triangle \infty 0$ & $<100$ & $<100$ & 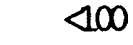 \\
\hline Pypere & NA & NA & $\mathrm{NA}$ & $<100$ & $<\infty$ & $<100$ & 400 \\
\hline Butylberzylphthalate & NA & NA & NA & $<100$ & $<00$ & $\triangle 100$ & 400 \\
\hline 3,3' -Dichlorobenzidene & NA & NA & $\mathrm{NA}$ & 200 & $<200$ & 210 & $<200$ \\
\hline Berzo(a)anthracere & M. & NA & NA & $<100$ & $<100$ & $<100$ & $<100$ \\
\hline Orrysene & $N_{11}$ & NA & $\mathrm{NA}$ & 400 & $\triangle \infty$ & $\triangle 100$ & 400 \\
\hline bis(2-Ethylhexyl)phthalate & NA & NA & NA & $4 \infty$ & $\triangle 100$ & $\triangle 100$ & $<100$ \\
\hline & & & & & & & \\
\hline
\end{tabular}


Table D.2 (continued)

\begin{tabular}{|c|c|c|c|c|c|c|c|}
\hline Sampling Date: & 27 sep89 & $275 e p 89$ & 27 sep 89 & 15 Feb 90 & $15 \mathrm{Feb} 90$ & 15 Feb 90 & $15 \mathrm{Feb} 90$ \\
\hline $\begin{array}{l}\text { Burial Tremch Nuber: } \\
\text { Species/Chemical: }\end{array}$ & $\begin{array}{r}T-2 \\
(\mathrm{ppb}) \\
\end{array}$ & $\begin{array}{l}T-3 \\
\text { (ppb) } \\
\end{array}$ & $\begin{array}{l}T-5 \\
(\mathrm{ppb})\end{array}$ & $\begin{array}{l}\text { T-11 } \\
\text { (ppb) }\end{array}$ & $\begin{array}{l}\mathrm{T}-13 \\
\text { (ppb) } \\
\end{array}$ & $\begin{array}{l}T-16 \\
\text { (ppb) }\end{array}$ & $\begin{array}{l}\text { T-252 } \\
\text { (ppb) }\end{array}$ \\
\hline Di-n-Octylphthalate & $\mathrm{NA}$ & NA & NA & 400 & 400 & $\varangle 00$ & 400 \\
\hline Berro(b) flionanthere & NA & NA & $N A$ & $\triangleleft 00$ & $<100$ & $<100$ & $<100$ \\
\hline Benroo(k) flumanthene & NA & NA & NA & 400 & $<\infty$ & 400 & 400 \\
\hline Berzo(a)pyrene & NA & NA & NA & $<00$ & $\triangle \infty 0$ & $\triangle 100$ & 400 \\
\hline Indero $(1,2,3-\infty d)$ pyrene & NA & NA & NA & 400 & 400 & $<100$ & $\triangle 00$ \\
\hline $\operatorname{Dibeng}(\mathrm{a}, \mathrm{h})$ anthracene & $\mathrm{NA}$ & NA & NA & $\triangle 100$ & 400 & $<100$ & 400 \\
\hline Berzo $(g, h, i)$ perylene & NA & NA & NA & $<00$ & 400 & 400 & 400 \\
\hline Acrylamide & $\mathrm{NA}$ & NA & NA & 1190 & $\triangleleft 0$ & 90 & $\triangle 0$ \\
\hline Silver & 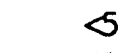 & $\sigma$ & 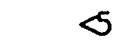 & 5 & 8 & $<$ & 6 \\
\hline Aluminm & $\diamond 0$ & $<0$ & $\diamond 0$ & 310 & 350 & 130 & $<0$ \\
\hline Arsenic & $<0$ & $<0$ & 50 & $<0$ & 54 & $<0$ & $\$ 0$ \\
\hline Boran & $<0$ & $<80$ & $<80$ & $\ll 0$ & $<80$ & $<80$ & $<8$ \\
\hline Barium & 160 & 61 & 100 & 290 & 210 & 150 & 230 \\
\hline Beryllium & $\diamond .4$ & $\varangle 0.4$ & $\varangle .4$ & $\varangle .3$ & $\varangle .3$ & $\varangle .3$ & $\varangle .3$ \\
\hline Calcium & 37000 & 27000 & 23000 & 130000 & 95000 & 110000 & 21000 \\
\hline Cadrium & 8 & $<$ & 8 & 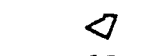 & $\triangleleft$ & $<$ & $\diamond$ \\
\hline Cobalt & 3 & $\beta$ & 5 & 15 & 17 & 24 & 40 \\
\hline Crmanim & 14 & 6 & 10 & 13 & 8 & 10 & 10 \\
\hline Copper & 25 & 23 & 26 & 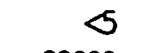 & 5 & $<$ & $<$ \\
\hline Iran & 130 & 280 & 410 & 39000 & 40000 & 30 & 17000 \\
\hline Merany & $\varangle .1$ & $\varangle .1$ & $\varangle .1$ & 0.1 & $\varangle .1$ & 0.3 & 0.3 \\
\hline Potassium & 1000 & 2300 & 3300 & 110000 & 2650 & 2230 & 2080 \\
\hline Lithium & $\triangle 5000$ & $\triangle 5000$ & $\triangle 5000$ & $\triangle 5000$ & $\triangle 15000$ & $\triangle 15000$ & 45000 \\
\hline Mgyesium & 6200 & 4700 & 4500 & 40000 & 26000 & 35000 & 12000 \\
\hline Manganese & 200 & 26 & 1900 & 5900 & 9300 & 4400 & 7900 \\
\hline Mblybdenum & 40 & 40 & 40 & 410 & 40 & 40 & 40 \\
\hline Sodium & 8000 & 8000 & $<2000$ & 30900 & 6540 & 4960 & 4690 \\
\hline Nidkel & 6 & 6 & 6 & $\theta$ & $\theta$ & $\theta$ & $\theta$ \\
\hline Phosphonus & $<00$ & $\infty 00$ & 300 & 47000 & $<00$ & $\infty 00$ & $<00$ \\
\hline Lead & $\infty 0$ & $<0$ & $\infty$ & $\infty 0$ & $\infty$ & $<0$ & $<0$ \\
\hline Antimany & 40 & 40 & 40 & 100 & 50 & $<0$ & 65 \\
\hline Selenium & $\varangle 8$ & $<80$ & $<80$ & 59 & 81 & 40 & 40 \\
\hline Silioan & 1600 & 1500 & 410 & 7700 & 11000 & 4600 & 4500 \\
\hline Tin & $<0$ & $<0$ & $<0$ & $<0$ & $<0$ & $<0$ & $<0$ \\
\hline Strantium & 59 & 41 & 42 & 200 & 170 & 170 & 80 \\
\hline Titanium & $\infty$ & $<20$ & $\infty 0$ & $<0$ & $\infty$ & 80 & $\infty 0$ \\
\hline Vanadium & $\infty$ & $<$ & $\infty$ & 4 & 4 & 4 & 4 \\
\hline Zinc & 16 & 24 & 25 & 150 & 58 & 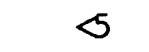 & 8 \\
\hline Ziroanium & $\infty$ & $<0$ & $<0$ & $<0$ & $\infty$ & $\infty$ & $<0$ \\
\hline Branide & $<0$ & $\diamond 0$ & $<0$ & $<00$ & $<0$ & $<0$ & $<0$ \\
\hline Chloride & 4650 & 4450 & 3360 & 50000 & 13300 & 11300 & 4270 \\
\hline Fluoride & 2400 & 330 & 2750 & 45000 & 2920 & 9720 & 780 \\
\hline Nitrate & 120 & 380 & 180 & $<00$ & 60 & 170 & 70 \\
\hline Thosphate & 150 & 340 & $<0$ & 66000 & $<0$ & $<0$ & $<0$ \\
\hline Sulfate & 7590 & 4760 & 10500 & 20000 & 6290 & 40100 & 5010 \\
\hline Total anganic Canban & 6900 & 8800 & 29300 & 615000 & 33000 & 362000 & 17000 \\
\hline
\end{tabular}


Table D.2 (continued)

\begin{tabular}{|c|c|c|c|c|c|c|c|}
\hline Sampling Date: & 27 sep89 & 27 Sep89 & 27 Sep89 & $15 \mathrm{Feb} 90$ & 15Feb90 & 15Feb90 & $15 F e b 90$ \\
\hline Brial Trench Number: & $\mathrm{T}-2$ & $\mathrm{~T}-3$ & $\mathrm{~T}-5$ & $T-11$ & $\mathrm{~T}-13$ & $T-16$ & $\mathrm{~T}-252$ \\
\hline \multirow[t]{2}{*}{ Species/themical: } & (ppb) & (ppb) & (pgb) & (ppb) & (pob) & (pob) & (pob) \\
\hline & $(\mathrm{Bq} / \mathrm{L})$ & $(\mathrm{Bq} / \mathrm{L})$ & $\overline{(\mathrm{Bg} / \mathrm{L})}$ & $\overline{(\mathrm{Bq} / \mathrm{L})}$ & $\overline{(\mathrm{Bg} / \mathrm{L})}$ & $(\mathrm{Bq} / \mathrm{L})$ & $(\mathrm{Bq} / \mathrm{L})$ \\
\hline Camban-14 & NA & NA & NA & 21 & 5 & 14 & 10 \\
\hline Cobalt-60 & NA & NA & NA & 0.5 & 0.6 & 0.6 & 0.1 \\
\hline Cesium-137 & NA & NA & NA & $\varangle .1$ & $\varangle .1$ & 0.2 & 1.2 \\
\hline Gross Alpha & NA & NA & $\mathrm{NA}$ & 0.032 & 0.029 & 0.057 & 0.035 \\
\hline Gross Beta & NA & NA & $\mathrm{NA}$ & 2.1 & 0.04 & 0.79 & 0.11 \\
\hline Strantium-90 & NA & NA & $\mathrm{NA}$ & 0.02 & 0.02 & 0.30 & $0 . \propto$ \\
\hline Tritium & 86 & 692 & 86 & 570 & 2500 & 1500 & 330 \\
\hline Americium-241 & NA & NA & $\mathrm{NA}$ & NA & $N A$ & NA & NA \\
\hline Arrium-244 & NA & NA & NA & $\mathrm{NA}$ & NA & NA & NA \\
\hline Plutanium-239 & NA & NA & NA & NA & NA & NA & NA \\
\hline Uranium-232 & NA & NA & NA & NA & $\mathrm{NA}$ & NA & NA \\
\hline Uranium-233 & NA & NA & NA & NA & NA & NA & NA \\
\hline $\mathrm{pH}$ & 7.2 & 7.2 & 7.3 & 7.2 & 7.4 & 7.1 & 7 \\
\hline Electrical Canductivity & 162 & 135 & 159 & 1823 & 594 & 609 & 249 \\
\hline Dissolved Solids & 200 & 180 & 240 & 1900 & 420 & 720 & 180 \\
\hline Total Solids & NA & N4 & $\mathrm{NA}$ & 2140 & 840 & 1060 & 660 \\
\hline Suspended Solids & NA & NA & NA & 240 & 420 & 340 & 480 \\
\hline Hanchess & 92 & 72 & 66 & 424 & 328 & 398 & 134 \\
\hline Alkalinity & 120 & 100 & 80 & 838 & 381 & 345 & 146 \\
\hline
\end{tabular}


Table D.3. Chamical analyses of SuSA 6 burial trench leachates

\begin{tabular}{|c|c|c|c|c|c|c|c|}
\hline Sampling Date: & $15 F e b 90$ & $15 \mathrm{Feb} 90$ & 15 Feb 90 & $15 \mathrm{Feb} 90$ & $15 \mathrm{Fe}$ 90 & $24 \pi u 190$ & 24Jul90 \\
\hline Burial Trench Number: & $\mathrm{T}-275$ & $\mathrm{~T}-279$ & $\mathrm{~T}-284$ & $\mathrm{~T}-285$ & $\mathrm{~T}-288$ & $\mathrm{~T}-13$ & $\mathrm{~T}-16$ \\
\hline Species/Chemical: & $(\mathrm{ppb})$ & (ppb) & (ppb) & (ppb) & (ppb) & (ppb) & (ppb) \\
\hline Chloramethane & $<000$ & $<000$ & $<000$ & $<000$ & $\triangle 10000$ & 40 & $<0$ \\
\hline Bromamethane & 5000 & $<000$ & $<000$ & $\$ 000$ & $\triangleleft 10000$ & $<0$ & $<0$ \\
\hline Vinyl Chloride & $<000$ & 5000 & $<000$ & $<000$ & $<10000$ & 40 & $<10$ \\
\hline Chlonoethane & $<000$ & $<000$ & $\measuredangle 000$ & $<000$ & $<10000$ & 40 & $<10$ \\
\hline Methylene chloride & $\mathrm{J} 520$ & $\mathrm{~J} 430$ & ת1000 & $\mathrm{J} 400$ & $\pi 790$ & $<$ & $<$ \\
\hline Acetane & 5700 & 8300 & 13000 & 50000 & $\triangleleft 0000$ & 16 & 37 \\
\hline Canbon disulfide & J890 & $<500$ & 2800 & 8500 & 5000 & $<$ & $<$ \\
\hline 1,1-Dichlonoethene & 8500 & 8500 & $<500$ & $<500$ & $\$ 500$ & $<$ & 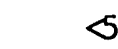 \\
\hline 1,1-Dichloroethane & 2500 & $<2500$ & 2500 & $<500$ & $\$ 000$ & $<$ & 5 \\
\hline 1,2-Dichlonoethene (Total) & 2500 & 8500 & 8500 & 8500 & $<000$ & 5 & $く$ \\
\hline Chlorsoform & $<500$ & $<500$ & $<2500$ & 2500 & 5000 & $<$ & 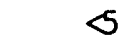 \\
\hline 1,2-Dichlonoethane & $<500$ & 8500 & 8500 & 8500 & 5000 & $<$ & $\triangleleft$ \\
\hline 2-Butanane & 5000 & $<5000$ & $<000$ & $\$ 000$ & $\triangleleft 10000$ & $\triangle 10$ & $<0$ \\
\hline 1,1,1-Trichllorøethane & $<500$ & 2500 & $<500$ & 8500 & $\$ 000$ & $<$ & $<$ \\
\hline Carban tetrachloride & 8500 & $<500$ & $<500$ & $<500$ & $<000$ & $<$ & $<$ \\
\hline Vinyl acetate & $<000$ & $<000$ & $<000$ & $\$ 000$ & $<0000$ & $\triangleleft 10$ & $<0$ \\
\hline Bramotichlonomethane & 2500 & 8500 & 8500 & 2500 & $<000$ & $\sigma$ & $<$ \\
\hline 1,2-Dichlomopropane & 2500 & $<500$ & 2500 & 8500 & $<000$ & $<$ & 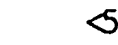 \\
\hline cis-1,3-Dichlomopropene & $<2500$ & 8500 & $<500$ & 2500 & $\$ 000$ & $<$ & 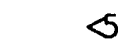 \\
\hline Trichllaroethene & 8500 & $<500$ & $<500$ & $<500$ & $\$ 000$ & 5 & $<$ \\
\hline Dibramochloramethane & $<500$ & 8500 & $<500$ & $<500$ & 5000 & $<$ & 5 \\
\hline 1,1,2-Trichloroethane & $<500$ & 8500 & $<500$ & $<500$ & $\$ 000$ & $<$ & 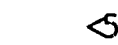 \\
\hline Berzene & $<500$ & $<500$ & 8500 & 8500 & $\measuredangle 000$ & 5 & 10 \\
\hline trans-1,3-Dichloropropene & 8500 & 8500 & $<500$ & 2500 & $<000$ & $<$ & $<$ \\
\hline Bramoform & $<500$ & 8500 & 8500 & 8500 & 5000 & $<$ & $<$ \\
\hline 4-Methyl-2-pentanane & $<000$ & $\$ 5000$ & $<000$ & $<000$ & $\triangle 10000$ & $<10$ & $<10$ \\
\hline 2-Hexanare & $<000$ & $\$ 500$ & $<000$ & $<000$ & $\triangle 10000$ & 40 & $<10$ \\
\hline Tetrachloroethene & $<2500$ & $<2500$ & $<500$ & $<500$ & 5000 & $<$ & 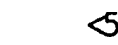 \\
\hline 1,1,2,2-tetrachloroethane & $<500$ & 8500 & $<500$ & 8500 & $<000$ & $<$ & $<$ \\
\hline Toluene & 150000 & 5000 & $<500$ & $<500$ & $<000$ & 18 & 37 \\
\hline Chloroberzene & $<500$ & 8500 & 2500 & 2500 & $<000$ & $<$ & 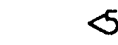 \\
\hline Ethylberzene & 3200 & $\sqrt{2} 300$ & $<500$ & 2500 & $<000$ & $<$ & 5 \\
\hline Styrene & $<500$ & 8500 & 8500 & 8500 & $<000$ & $\infty$ & 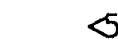 \\
\hline Xylene (Total) & $\sqrt{1500}$ & $<500$ & 8500 & 8500 & $\$ 000$ & 486 & 56600 \\
\hline Fhenol & $4 \infty$ & $<100$ & $<\infty$ & $\triangle 00$ & $\triangle 00$ & NA & NA \\
\hline bis(2-ahloroethyl)ether & $\triangle 100$ & $\triangle 00$ & $\triangleleft \infty 0$ & $\triangle 100$ & $\varangle 100$ & NA & $\mathrm{NA}$ \\
\hline 2-Chlorophenol & $\triangleleft \infty 0$ & $\triangle 00$ & $4 \infty$ & 400 & $\triangle 100$ & NA & NA \\
\hline 1,3-Dichlondberzene & 400 & $<00$ & 400 & $\triangleleft 100$ & $\triangleleft 00$ & NA & $\mathrm{NA}$ \\
\hline 1,4-Dichlonobenzene & $\triangleleft 00$ & $\triangleleft 100$ & 400 & $<100$ & $\varangle 00$ & NA & $\mathrm{NA}$ \\
\hline Berzyl alochol & 400 & 400 & $\triangle \infty 0$ & $\triangleleft 00$ & $\triangle 100$ & NA & NA \\
\hline 1,2-Dichloroberzene & $\triangleleft 100$ & $<100$ & 400 & $\triangle 100$ & $\triangle 00$ & NA & $\mathrm{NA}$ \\
\hline 2-Methylphenol & 400 & $<100$ & $<100$ & $\triangleleft 100$ & $\varangle 100$ & NA & $\mathrm{NA}$ \\
\hline bis(2-Chloroisopropyl)ether & $\triangleleft 100$ & $\triangleleft \infty$ & $<10$ & $\triangleleft \infty 0$ & $\varangle 100$ & NA & $\mathrm{Na}$ \\
\hline 4-Methylphenol & $\triangleleft \infty 0$ & $\triangleleft 100$ & $<100$ & $\triangle 100$ & $\varangle 00$ & NA & NA \\
\hline N-ritroso-di-n-propylamine & $\triangleleft 00$ & $<100$ & $<100$ & $\triangle 100$ & $\triangle 100$ & NA & NA \\
\hline Hexachloroethane & $\triangleleft 100$ & 400 & $<100$ & $\triangle 00$ & $\varangle \infty$ & NA & NA \\
\hline
\end{tabular}


Table D.3 (continued)

\begin{tabular}{|c|c|c|c|c|c|c|c|}
\hline $\begin{array}{l}\text { Sampling Date: } \\
\text { Burial Trench Number: } \\
\text { Species/Chemical: }\end{array}$ & $\begin{array}{r}15 \mathrm{Feb} 90 \\
\mathrm{~T}-275 \\
\text { (ppb) } \\
\end{array}$ & $\begin{array}{r}15 \text { Feb } 90 \\
\mathrm{~T}-279 \\
\text { (ppb) } \\
\end{array}$ & $\begin{array}{r}15 F e b 90 \\
\mathrm{~T}-284 \\
(\mathrm{ppb}) \\
\end{array}$ & $\begin{array}{r}15 F e b 90 \\
\text { T-285 } \\
\text { (ppb) } \\
\end{array}$ & $\begin{array}{r}15 F e b 90 \\
T-288 \\
\text { (ppb) } \\
\end{array}$ & $\begin{array}{c}24 \mathrm{Ju} 190 \\
\mathrm{~T}-13 \\
\text { (ppb) }\end{array}$ & $\begin{array}{c}24 \mathrm{Ju} 190 \\
\mathrm{~T}-16 \\
\text { (ppb) }\end{array}$ \\
\hline Nitrabernene & 400 & 400 & $4 \infty$ & $\triangle 100$ & $\triangle 00$ & $\mathrm{NA}$ & \\
\hline Iscphonane & $\triangle 10$ & $\triangleleft \infty 0$ & $\triangle 100$ & $\triangleleft 00$ & 400 & NA & \\
\hline 2-Nitrophemol & 400 & $\triangle 100$ & $<00$ & $<100$ & $\triangleleft \infty$ & NA & \\
\hline 2,4-Dimethylphenol & 400 & $\triangleleft 00$ & 400 & 400 & $\triangleleft 00$ & $\mathrm{NA}$ & \\
\hline Berzoic acid & $\mathrm{J}_{4}$ & ఠ10 & $\diamond 10$ & $<10$ & $\$ 10$ & NA & \\
\hline bis(2-chlonoethaxy)methane & 400 & $4 \infty$ & $<100$ & $\triangle 00$ & $\triangle 100$ & NA & \\
\hline 2,4-Dichlorophenol & $\triangleleft 100$ & $\triangleleft \infty$ & $4 \infty$ & $<\infty$ & $<100$ & NA & \\
\hline 1,2,4-Trichlandberzene & 400 & 400 & $<\infty$ & 400 & $\triangle 100$ & NA & \\
\hline Nephthalene & 820 & 810 & 400 & $<100$ & $\sqrt{32}$ & $\mathrm{NA}$ & \\
\hline 4-chlonoaniline & 400 & $\triangleleft 00$ & 400 & 400 & 400 & NA & \\
\hline Hexachlorobutactiene & $\triangle \infty 0$ & $\triangleleft 100$ & 400 & $\triangle 00$ & $\triangle 100$ & NA & \\
\hline 4-ahloro-3-methylphemol & $\triangleleft \infty 0$ & $\triangleleft 00$ & 400 & $<100$ & $\triangle 00$ & NA & \\
\hline 2-Methylnet & 400 & $\triangleleft \infty 0$ & $\triangle 100$ & $<00$ & $\triangle 100$ & NA & \\
\hline lopentadiene & $\triangleleft \infty 0$ & $\triangle 100$ & $\triangle 00$ & $<100$ & 400 & NA & \\
\hline 2,4,6-Trichlono & 400 & $\triangleleft 00$ & 400 & 400 & 400 & NA & \\
\hline 2,4,5-Trichlonophenol & $\delta 10$ & 810 & $\diamond 10$ & $<10$ & $\checkmark 10$ & NA & \\
\hline 2-Chloranaphthalene & $\triangle \infty 0$ & 400 & $\triangle \infty 0$ & $\triangle \infty 0$ & $\triangle 100$ & NA & \\
\hline 2-Nitromiline & $\delta 10$ & $\checkmark 10$ & $<10$ & $<10$ & 510 & NA & \\
\hline Dimethylphthalate & $4 \infty 0$ & $<\infty$ & $<100$ & $<100$ & $\triangle 100$ & NA & \\
\hline lene & $\triangle \infty 0$ & $<\infty$ & $\triangle 100$ & $<\infty$ & $<100$ & NA & \\
\hline 2,6-Dinitrotaluene & 400 & $\Delta 00$ & 400 & $<100$ & $<00$ & NA & \\
\hline 3-Nitroentline & $<10$ & 510 & $\delta 10$ & $\sigma 10$ & 510 & NA & \\
\hline Acena & 400 & $<00$ & $\triangle 00$ & $<\infty$ & $\triangle 100$ & NA & \\
\hline 2,4-Dinitrophenol & $\delta 10$ & $\sigma 10$ & $<10$ & $<10$ & $\$ 10$ & NA & \\
\hline 4-Nitrophenol & $\delta 10$ & s10 & 510 & $<10$ & $<10$ & NA & \\
\hline Diberzofiran & $\triangleleft 00$ & $\triangleleft \infty$ & $\triangle 100$ & $<100$ & $<00$ & NA & \\
\hline toluare & 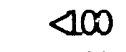 & $\Delta \alpha$ & 400 & $\triangle \infty 0$ & $<100$ & NA & \\
\hline Diethylphthalate & 400 & 400 & 400 & $<100$ & $\triangleleft 100$ & NA & \\
\hline 4-Qhlonopheryl-phenylether & 400 & 400 & 400 & 400 & $<00$ & NA & \\
\hline Fluorene & 400 & 400 & 400 & 400 & $\triangleleft 100$ & NA & \\
\hline 4-Nitroaniline & $\delta 10$ & 810 & 510 & $<10$ & $\diamond 10$ & NA & \\
\hline 4,6-Dinitro-2-methylphenol & 510 & 510 & 510 & $<10$ & $\diamond 10$ & NA & \\
\hline N-nitrosodiphenylamine & 400 & $\triangleleft \infty 0$ & 400 & $<100$ & 400 & NA & \\
\hline nylether & 400 & $\triangle 00$ & $<100$ & $<100$ & $\triangleleft 00$ & NA & \\
\hline Hexachlonoberzene & $\triangleleft 00$ & $\triangleleft \infty$ & $4 \infty$ & $\triangle \infty 0$ & $<100$ & NA & \\
\hline Pentachloraphenol & $\delta 10$ & 510 & 510 & $<10$ & 510 & NA & \\
\hline Phenanthrene & $\triangleleft 00$ & $\triangleleft \infty 0$ & 400 & $\triangle 100$ & 400 & NA & \\
\hline Anthreacene & $\triangleleft 00$ & $\triangleleft \infty 0$ & $\triangle 100$ & $<00$ & 400 & NA & \\
\hline Di-n-butylphthalate & $\triangle 100$ & $\triangleleft 00$ & 400 & $<100$ & $<100$ & NA & \\
\hline Fluoranthere & 400 & $\triangleleft \infty 0$ & $\triangle 100$ & $<\infty$ & $\triangle \infty$ & NA & \\
\hline Pyrene & 400 & $\triangle 100$ & $\triangle 100$ & $<00$ & $<100$ & NA & \\
\hline Butylbenzylp & 400 & 400 & 400 & 400 & $\triangleleft 100$ & NA & \\
\hline 3,3' -Dichlondbenzidene & $<200$ & $\infty 00$ & 800 & 800 & $<00$ & NA & \\
\hline Berzo(a)anthracene & 400 & 800 & 400 & $\triangle 100$ & 400 & NA & \\
\hline Onrysene & 400 & $\triangle 00$ & 400 & $\triangle 100$ & 400 & NA & \\
\hline bis(2-Ethylhexyl)phthalate & $\triangle 100$ & $\triangle 00$ & 400 & $<00$ & 400 & NA & \\
\hline
\end{tabular}


Table D.3 (ontinued)

\begin{tabular}{|c|c|c|c|c|c|c|c|}
\hline Sampling Date: & $15 F B b 90$ & $15 F e b 90$ & $15 \mathrm{Feb} 90$ & 15Feb90 & $15 \mathrm{Feb} 90$ & $24 \mathrm{Ju} 190$ & $24 \pi u 190$ \\
\hline $\begin{array}{l}\text { Burial Trench Nutier: } \\
\text { Species/themical: }\end{array}$ & $\begin{array}{l}\mathrm{T}-275 \\
\text { (ppb) }\end{array}$ & $\begin{array}{l}\mathrm{T}-279 \\
(\mathrm{pab})\end{array}$ & $\begin{array}{c}\mathrm{T}-284 \\
(\mathrm{ppb})\end{array}$ & $\begin{array}{l}\mathrm{T}-285 \\
(\mathrm{ppb})\end{array}$ & $\begin{array}{c}\mathrm{T}-288 \\
\text { (ppb) }\end{array}$ & $\begin{array}{l}\mathrm{T}-13 \\
(\mathrm{ppb})\end{array}$ & $\begin{array}{l}\mathrm{T}-16 \\
(\mathrm{ppb})\end{array}$ \\
\hline Di-n-Octyly hthalate & 400 & 400 & $<100$ & 400 & 400 & NA & NA \\
\hline Berro(b) flumranthere & $\varangle 00$ & $\triangle 100$ & $\triangleleft 00$ & $<100$ & $<100$ & NA & NA \\
\hline Berzo(k)fluoranthene & $\triangleleft 00$ & $\triangleleft 00$ & $<00$ & $<00$ & $<00$ & NA & NA \\
\hline Berzo(a)pyrene & $\varangle 00$ & 400 & $\triangleleft 00$ & $<100$ & 400 & $\mathrm{NA}$ & NA \\
\hline Indemo $(1,2,3-\infty)$ pyrene & $\triangleleft \infty$ & $\triangle 100$ & $\triangle 00$ & 400 & $\triangleleft 00$ & $\mathrm{NA}$ & NA \\
\hline Dibenz $(\mathrm{a}, \mathrm{h})$ andtracene & $\triangleleft 00$ & $\triangle 100$ & $\triangle 100$ & $\triangle 00$ & $<100$ & NA & NA \\
\hline $\operatorname{Berzo}(\mathrm{g}, \mathrm{h}, \mathrm{i})$ perylene & $\triangle 00$ & 100 & $<100$ & $\Delta 00$ & $\Delta 00$ & NA & NA \\
\hline Acrylamide & $<0$ & $<10$ & 40 & 40 & 40 & $\mathbb{N A}$ & NA \\
\hline Silver & 5 & 5 & 5 & $\infty$ & 5 & NA & NA \\
\hline Aluminum & $<0$ & 130 & 440 & 180 & $\infty 0$ & NA & NA \\
\hline Arsenic & $<0$ & 63 & $<0$ & $<5$ & $<0$ & NA & NA \\
\hline Bonan & $\varangle 0$ & $<80$ & $<80$ & $<0$ & $\ll 80$ & NA & NA \\
\hline Barium & 130 & 130 & 170 & 130 & 160 & NA & NA \\
\hline Beryllium & $\varangle .3$ & $\$ .3$ & $\varangle .3$ & $\varangle .3$ & $\varangle .3$ & $\mathrm{NA}$ & NA \\
\hline Calcium & 39000 & 110000 & 120000 & 110000 & 64000 & NA & NA \\
\hline Cachium & $\diamond$ & $\triangleleft$ & $\triangleleft$ & $<$ & $\diamond$ & NA & NA \\
\hline Cobalt & 43 & 10 & $\varangle$ & $\varangle$ & 21 & $\mathrm{NA}$ & NA \\
\hline Amranium & 6 & 8 & 5 & 5 & 6 & NA & NA \\
\hline Copper & 5 & 5 & 5 & $\diamond$ & $\diamond$ & NA & NA \\
\hline Iran & 190 & 16000 & 140 & 27 & 7900 & NA & NA \\
\hline Merary & $\$ .1$ & 0.3 & 0.4 & $ه .1$ & 2120 & NA & NA \\
\hline Potassium & 730 & 1960 & 800 & 530 & 3300 & NA & NA \\
\hline Lithium & $<15000$ & $\triangleleft 5000$ & 45000 & $\triangle 15000$ & $\triangle 15000$ & NA & NA \\
\hline Magnesium & 14000 & 26000 & 32000 & 15000 & 18000 & $\mathrm{NA}$ & $\mathrm{NA}$ \\
\hline Manganese & 3600 & 6100 & 600 & 260 & 3700 & NA & NA \\
\hline Molybdenim & 40 & $\varangle 0$ & $\$ 0$ & 40 & 40 & NA & NA \\
\hline Soctium & 1650 & 3010 & 2530 & 1040 & 1200 & NA & NA \\
\hline Nickel & $\diamond$ & $\theta$ & $\theta$ & $\diamond$ & $\theta$ & NA & NA \\
\hline Phosphonus & 300 & $\infty 00$ & 300 & $<00$ & 800 & $\mathrm{NA}$ & NA \\
\hline Lead & $\infty 0$ & $<0$ & $<30$ & $\infty 0$ & $\infty 0$ & NA & NA \\
\hline Antimany & $<0$ & 66 & $<0$ & $\measuredangle 0$ & 51 & NA & NA \\
\hline Selenium & 40 & 40 & $\varangle 0$ & $\varangle 0$ & $\varangle 0$ & $\mathrm{NA}$ & NA \\
\hline Silioan & 2200 & 5100 & 890 & 880 & 2900 & NA & $\mathrm{NA}$ \\
\hline Tin & $<0$ & $<5$ & $<0$ & $\measuredangle 0$ & $<0$ & NA & $\mathrm{NA}$ \\
\hline Strantium & 81 & 170 & 170 & 130 & 120 & NA & NA \\
\hline Titanium & $\infty$ & $<0$ & $\infty$ & $<0$ & $\infty$ & NA & NA \\
\hline Vanadium & 4 & 4 & 4 & 4 & 4 & $\mathrm{NA}$ & NA \\
\hline $\operatorname{Zin}$ & 1.7 & 58 & 560 & 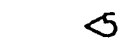 & 320 & NA & $\mathrm{NA}$ \\
\hline Ziroonium & $\infty$ & $<0$ & $\infty$ & $<0$ & $\infty 0$ & NA & NA \\
\hline Bramide & $\measuredangle 0$ & 70 & $\varsigma 0$ & $\diamond 0$ & $<0$ & NA & NA \\
\hline Chloride & 2220 & 4610 & 5090 & 1150 & 2540 & NA & NA \\
\hline Fluoride & 1600 & 1090 & 190 & 200 & 140 & NA & NA \\
\hline Nitrate & 80 & 110 & 110 & 170 & 130 & $\mathrm{NA}$ & NA \\
\hline Hosphate & $<0$ & $<5$ & $<0$ & $<0$ & $<0$ & NA & $\mathrm{NA}$ \\
\hline Sulfate & 10500 & 1390 & 7320 & 6130 & 820 & NA & $N A$ \\
\hline Total Organic Camban & 17000 & 22000 & 8800 & 4000 & 7500 & $M$ & $\mathrm{NA}$ \\
\hline
\end{tabular}


Table D.3 (continued)

\begin{tabular}{|c|c|c|c|c|c|c|c|}
\hline $\begin{array}{l}\text { Sampling Date: } \\
\text { Burial Trench Nuber: } \\
\text { Species/themical: }\end{array}$ & $\begin{array}{r}15 \mathrm{Feb} 90 \\
\mathrm{~T}-275 \\
\text { (ppb) } \\
\end{array}$ & $\begin{array}{r}15 \mathrm{Feb} 90 \\
\mathrm{~T}-279 \\
\text { (ppb) } \\
\end{array}$ & $\begin{array}{r}15 \text { Feb } 90 \\
\mathrm{~T}-284 \\
\text { (ppb) } \\
\end{array}$ & $\begin{array}{r}15 F e b 90 \\
\text { T-285 } \\
\text { (ppb) } \\
\end{array}$ & $\begin{array}{r}15 F e b 90 \\
\mathrm{~T}-288 \\
\text { (ppb) } \\
\end{array}$ & $\begin{array}{l}\text { 24Ju190 } \\
\mathrm{T}-13 \\
\text { (ppb) }\end{array}$ & $\begin{array}{l}24 \sqrt{ }{ }^{2} 190 \\
\mathrm{~T}-16 \\
\text { (pob) }\end{array}$ \\
\hline Canban-14 & $(\mathrm{Bq} / \mathrm{L})$ & $(\mathrm{Bq} / \mathrm{L})$ & $(\mathrm{Bq} / \mathrm{L})$ & $(B g / L)$ & $(\mathrm{Bq} / \mathrm{L})$ & $(B q / L)$ & $(\mathrm{Bg} / \mathrm{L})$ \\
\hline Cobalt-60 & $\begin{array}{r}8 \\
1.2\end{array}$ & $ه 1^{3}$ & 8 & 2 & 4 & NA & NA \\
\hline Cesium-137 & $\$ .1$ & $\$ .1$ & $\varangle .1$ & $\begin{array}{l}1.6 \\
0.8\end{array}$ & $<.1$ & NA & NA \\
\hline Gross Alpha & 0.049 & 0.035 & $\begin{array}{l}\$ 0.1 \\
0.058\end{array}$ & $\begin{array}{r}0.8 \\
0.076\end{array}$ & $\infty .1$ & $\begin{array}{ll}M A \\
N A\end{array}$ & NA \\
\hline Gross Beta & 0.01 & 0.10 & 0.43 & 0.28 & 0.54 & $\begin{array}{l}\text { NA } \\
\text { NA }\end{array}$ & $\begin{array}{l}\text { NA } \\
\text { NA }\end{array}$ \\
\hline Strantium-90 & 0.01 & 0.02 & $\varangle .01$ & 0.06 & 0.09 & NA & NA \\
\hline Tritium & 100 & 300 & 430 & 94 & 350 & NA & NA \\
\hline Americium-241 & NA & NA & NA & NA & NA & NA & NA \\
\hline Arrium-244 & $\mathrm{NA}$ & NA & NA & NA & NA & NA & $\mathbf{N A}$ \\
\hline Plutanium-239 & $\mathrm{NA}$ & NA & $N A$ & NA & NA & NA & NA \\
\hline Uranium-232 & NA & NA & $\mathrm{NA}$ & NA & NA & NA & $\mathrm{NA}$ \\
\hline Uranium-233 & $\mathrm{NA}$ & NA & $\mathrm{NA}$ & NA & NA & NA & NA \\
\hline $\mathrm{pH}$ & 7.4 & 7 & 7.9 & 7.8 & 6.9 & 6.4 & 6.6 \\
\hline Electrical Conductivity & 266 & 511 & 590 & 490 & 373 & 748 & 935 \\
\hline Dissolved Solids & 240 & 552 & 500 & 380 & 320 & 380 & 640 \\
\hline Total Solids & 4160 & 1080 & 1620 & 2040 & 740 & NA & $N A$ \\
\hline Suspended Solids & 3920 & 528 & 1120 & 1660 & 420 & NA & NA \\
\hline Handhess & 158 & 512 & 396 & 308 & 206 & 328 & 512 \\
\hline Alkalinity & 159 & 482 & 42 & 331 & 236 & 396 & 560 \\
\hline
\end{tabular}


Thble D.4. Chemical analyses of SHSA 6 burial trench leachates

\begin{tabular}{|c|c|c|c|c|c|c|c|}
\hline Sampling Date: & 24Jul90 & $24 \sqrt{190} 2$ & 24Jul90 & 24Jul90 & 24Jul90 & $24 \pi u 190$ & $24 \pi u 190$ \\
\hline Burial Trench Nuber: & T-11 & T-288 & T-285 & T-252 & T-284 & T-275 & $\mathrm{T}-279$ \\
\hline Species/themical: & & & & & (ppb) & (ppb) & (ppb) \\
\hline Chlonamethane & 40 & 40 & 40 & 40 & 40 & $\triangleleft 0$ & $<10$ \\
\hline Bromomethene & $\triangle 0$ & 40 & $<10$ & $\triangle 0$ & $\Delta 0$ & 40 & $\triangle 0$ \\
\hline Vinyl Chloride & $\triangleleft 0$ & $<0$ & $<0$ & 40 & 40 & 40 & $<0$ \\
\hline Chlonoethane & $\triangleleft 0$ & $<0$ & $<0$ & 40 & $<0$ & $<0$ & $<0$ \\
\hline Methylene chloride & $\varsigma$ & $\infty$ & 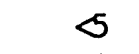 & 5 & 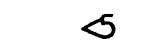 & 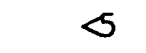 & $\delta$ \\
\hline Acetane & 3270 & 108 & $\triangleleft 0$ & J5 & $\pi$ & $\sqrt{3}$ & $\mathrm{~J} 3$ \\
\hline Canban disulfide & $\delta$ & 5 & $\sigma$ & $\zeta$ & $<$ & 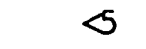 & 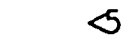 \\
\hline 1,1-Dichlomoethene & 5 & $\diamond$ & 5 & 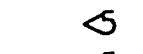 & $<$ & $<$ & $\diamond$ \\
\hline 1,1-Dichlonoethane & $\delta$ & 5 & $<$ & 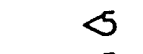 & $\infty$ & $<$ & $<$ \\
\hline 1,2-Dichloroethene (Total) & 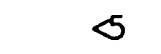 & $<$ & 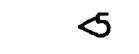 & 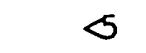 & $\delta$ & 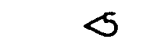 & 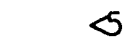 \\
\hline Chloroform & 5 & $<$ & $<$ & 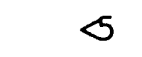 & $<$ & 5 & $<$ \\
\hline 1,2-Dichloroethane & $\delta$ & 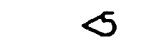 & $<$ & 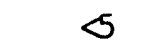 & 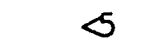 & 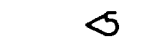 & 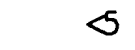 \\
\hline 2-Butanane & 40 & $<0$ & 40 & $\triangleleft 0$ & $\triangleleft 0$ & 40 & $<10$ \\
\hline 1,1,1-Trichlomothane & 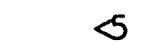 & $<$ & 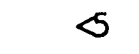 & 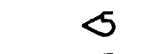 & $\diamond$ & $<$ & 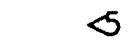 \\
\hline Canban tetrachloride & 5 & 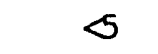 & 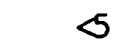 & 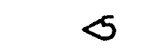 & $<$ & 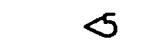 & 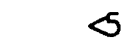 \\
\hline Vinyl acetate & $\triangle 0$ & $\triangle 0$ & $\triangle 0$ & $\varangle 0$ & $\triangle 10$ & $\triangle 10$ & $<10$ \\
\hline Bromodichlorcmethane & $\delta$ & $\sigma$ & $<$ & $<$ & $\delta$ & $<$ & 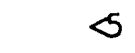 \\
\hline 1,2-Dichlonopropane & 5 & j & $<$ & 5 & $\delta$ & 5 & 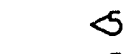 \\
\hline cis-1,3-Dichloropropene & 5 & 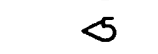 & 5 & 5 & $<$ & 5 & $<$ \\
\hline Trichlarcethene & $<$ & $<$ & $<$ & 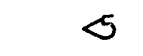 & $<$ & $<$ & 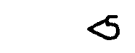 \\
\hline Dibramochlorcmethane & 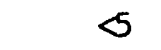 & $s$ & $\delta$ & $\sigma$ & $<$ & 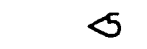 & 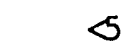 \\
\hline 1,1,2-Trichloroethane & $\delta$ & 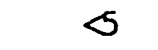 & $\diamond$ & $\sigma$ & $\diamond$ & 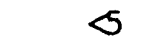 & $<$ \\
\hline Berzene & 9 & $\delta$ & $<$ & $<$ & $\diamond$ & $<$ & $\mathrm{J} 2$ \\
\hline trans-1,3-Dichlonopropene & $\delta$ & $<$ & 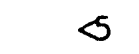 & $<$ & $<$ & $<$ & $\sigma$ \\
\hline Bramofom & 5 & 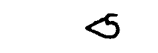 & $<$ & 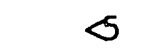 & 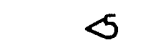 & 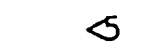 & $<$ \\
\hline 4-Methyl-2-pentanane & $<0$ & $<0$ & $\triangle 0$ & $\triangle 0$ & $\triangle 0$ & $\triangle 10$ & $<0$ \\
\hline 2-Hexanane & $\triangle 10$ & $\triangle 0$ & $\triangleleft n$ & 40 & $\triangle 0$ & $\varangle 10$ & $<0$ \\
\hline Tetrachlonoethene & 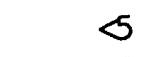 & $<$ & $<5$ & $<$ & $\diamond$ & $\diamond$ & 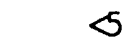 \\
\hline 1,1,2,2-tetrachlomothane & 5 & $<$ & 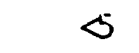 & $<$ & 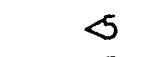 & 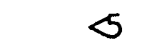 & 5 \\
\hline Toluene & 23 & 15 & $\diamond$ & 20 & 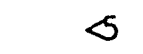 & $\mathrm{J} 2$ & $J 4$ \\
\hline Ohlordberzene & 5 & 5 & 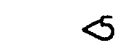 & 5 & $<$ & 5 & $<$ \\
\hline Ethylbenzene & $<$ & $<$ & 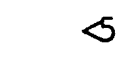 & $\diamond$ & $\varsigma$ & 5 & $\infty$ \\
\hline Styrene & $\diamond$ & 5 & 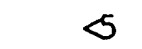 & 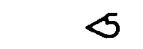 & $\diamond$ & $<$ & $<$ \\
\hline Xylene (Total) & 9000 & 7300 & $\mathrm{~J} 2$ & 28 & $\pi$ & 60 & 42300 \\
\hline Fhenol & NA & NA & $N A$ & NA & NA & NA & NA \\
\hline bis(2-olloroethyl)ether & NA & NA & NA & NA & $\mathrm{NA}$ & NA & $\mathrm{NA}$ \\
\hline 2-Chlonghenol & NA & NA & $\mathrm{NA}$ & NA & NA & NA & NA \\
\hline 1,3-Dichloroberzene & NA & NA & NA & NA & NA & NA & NA \\
\hline 1,4-Dichlonoberzene & NA & NA & NA & NA & NA & NA & NA \\
\hline Berzyl alochol & NA & NA & NA & NA & NA & NA & NA \\
\hline 1,2-Dichlondberzene & NA & NA & NA & NA & $\mathrm{NA}$ & NA & $\mathrm{NA}$ \\
\hline 2-Methylphenol & $\mathrm{NA}$ & NA & NA & NA & $\mathrm{NA}$ & NA & NA \\
\hline bis(2-chlonoisopropyl)ether & NA & NA & NA & $\mathrm{NA}$ & NA & NA & NA \\
\hline 4-Methylphenol & NA & NA & NA & $N A$ & NA & NA & NA \\
\hline N-nitroso-di-n-propylamine & $\mathrm{NA}$ & NA & $\mathrm{NA}$ & NA & NA & NA & NA \\
\hline Hexachioroethane & in & in & in & NÁ & in & Mn & NA \\
\hline
\end{tabular}


Table D.4 (cantinued)

\begin{tabular}{|c|c|c|c|c|c|c|c|}
\hline Sampling Date: & 24Jul90 & $24 \pi u 190$ & 24Jul90 & 24Ju190 & 24Ju190 & 24Ju190 & $24 \pi u 190$ \\
\hline Burial Trench Nuber: & $\mathrm{T}-11$ & T-288 & $T-285$ & $\mathrm{~T}-252$ & $\mathrm{~T}-284$ & $\mathrm{~T}-275$ & $\mathrm{~T}-279$ \\
\hline Species thamical: & (ppb) & $(\mathrm{ppb})$ & (ppb) & (ppb) & (ppb) & (ppb) & (ppb) \\
\hline Nitroberzene & $\mathrm{NA}$ & NA & $\sqrt{N A}$ & $\overline{\mathrm{NA}}$ & $\overrightarrow{N A}$ & $\overline{N A}$ & $\mathrm{NA}$ \\
\hline Ispothrane & $\mathrm{NA}$ & NA & NA & NA & NA & $\mathrm{NA}$ & NA \\
\hline 2-Nitrophenol & $N A$ & NA & NA & NA & NA & $\mathrm{NA}$ & $\mathrm{NA}$ \\
\hline 2,4-Dimethylphenal & NA & NA & NA & NA & NA & NA & NA \\
\hline Berzoic acid & NA & NA & NA & NA & NA & NA & NA \\
\hline bis(2-chlonoethaxy)methane & NA & NA & NA & NA & $\mathrm{NA}$ & NA & NA \\
\hline 2,4-Dichlorophenol & $\mathrm{NA}$ & $\mathrm{NA}$ & NA & NA & NA & NA & NA \\
\hline 1,2,4-Trichloroberzene & NA & NA & NA & NA & NA & NA & NA \\
\hline Nephthalene & NA & NA & $N A$ & NA & NA & NA & NA \\
\hline 4-chloroandline & NA & NA & NA & NA & NA & NA & NA \\
\hline Hexachlorobutadiene & $\mathrm{NA}$ & NA & NA & NA & $\mathrm{NA}$ & NA & NA \\
\hline 4-Chloro-3-methylphenol & NA & NA & NA & NA & NA & NA & NA \\
\hline 2-Methylnephthalene & $\mathrm{NA}$ & NA & $\mathrm{NA}$ & NA & NA & NA & NA \\
\hline Hexachlorocyclopentadiene & NA & NA & NA & $\mathrm{NA}$ & NA & NA & NA \\
\hline 2,4,6-Trichlorquhemol & NA & NA & NA & IA & NA & NA & NA \\
\hline 2,4,5-Trichlaropherol & $\mathrm{NA}$ & $\mathrm{NA}$ & NA & NA & $\mathrm{NA}$ & NA & NA \\
\hline 2-Chloranaphthalene & NA & NA & NA & NA & NA & NA & NA \\
\hline 2-Nitroenuline & $\mathrm{NA}$ & NA & NA & NA & NA & NA & NA \\
\hline Dimethylphthalate & NA & NA & NA & NA & $\mathrm{NA}$ & NA & NA \\
\hline Acenaphthylere & NA & NA & NA & NA & NA & $\mathrm{NA}$ & NA \\
\hline 2,6-Dinitrotoluene & NA & NA & NA & NA & NA & NA & NA \\
\hline 3-Nitroemiline & NA & NA & NA & NA & NA & NA & NA \\
\hline Aoenaphthere & NA & NA & NA & NA & NA & $\mathrm{NA}$ & NA \\
\hline 2,4-Dinitraphenol & NA & NA & NA & $\mathrm{NA}$ & NA & NA & NA \\
\hline 4-Nitrophenol & NA & NA & NA & NA & NA & NA & NA \\
\hline Dibenzofiucan & NA & NA & NA & NA & $\mathrm{NA}$ & NA & NA \\
\hline 2,4-Dinitrotoluene & NA & $\mathrm{NA}$ & NA & NA & NA & NA & NA \\
\hline Diethylphthalate & $\mathrm{NA}$ & NA & NA & $\mathbb{N A}$ & NA & NA & NA \\
\hline 4-Chlorophenyl-phenylether & $\mathrm{NA}$ & NA & $\mathrm{NA}$ & $\mathrm{NA}$ & NA & $\mathrm{NA}$ & NA \\
\hline Fluorene & NA & NA & NA & NA & NA & NA & NA \\
\hline 4-Nitroaniline & NA & NA & NA & NA & NA & NA & $\mathrm{NA}$ \\
\hline 4,6-Dinitro-2-methylyhenol & $\mathrm{NA}$ & NA & $\mathrm{NA}$ & NA & NA & $\mathrm{NA}$ & NA \\
\hline N-nitrosotiphenylamine & NA & NA & $\mathrm{NA}$ & NA & NA & NA & $\mathrm{NA}$ \\
\hline 4-bramophenyl-phenylether & NA & NA & NA & NA & NA & NA & $N A$ \\
\hline Hexachloroberzene & NA & NA & $\mathrm{NA}$ & NA & NA & NA & NA \\
\hline Pentachlanophenol & NA & NA & NA & NA & $\mathrm{NA}$ & NA & $\mathrm{NA}$ \\
\hline Phenanthene & $\mathrm{NA}$ & NA & $\mathrm{NA}$ & NA & $\mathrm{NA}$ & NA & NA \\
\hline Anthracene & NA & NA & NA & NA & NA & NA & NA \\
\hline Di-n-butylphthalate & NA & NA & NA & NA & NA & NA & NA \\
\hline Fluoranthene & $\mathrm{NA}$ & NA & $\mathrm{NA}$ & NA & NA & $\mathrm{NA}$ & $\mathrm{NA}$ \\
\hline Pyrene & NA & NA & NA & NA & NA & NA & $\mathrm{NA}$ \\
\hline Butylbenzylphthalate & $\mathrm{NA}$ & NA & $\mathrm{NA}$ & NA & NA & NA & NA \\
\hline 3,3' -Dichlandbenzidene & NA & NA & NA & NA & NA & NA & NA \\
\hline Berzo(a)anthracere & $\mathrm{NA}$ & NA & NA & NA & NA & NA & NA \\
\hline Orrysene & $\mathrm{NA}$ & NA & NA & NA & NA & NA & NA \\
\hline bis(2-Ethylhexyl)ghthalate & $\mathrm{NA}$ & NA & NA & NA & NA & NA & NA \\
\hline
\end{tabular}


Table D.4 (ontinued)

\begin{tabular}{|c|c|c|c|c|c|c|c|}
\hline $\begin{array}{l}\text { Sampling Date: } \\
\text { Burial Thench Number: } \\
\text { Species/Chemical: }\end{array}$ & $\begin{array}{c}24 \mathrm{Ju} 190 \\
\mathrm{~T}-11 \\
\text { (ppb) } \\
\end{array}$ & $\begin{array}{r}24 \pi u 190 \\
\text { T-288 } \\
\text { (ppb) } \\
\end{array}$ & $\begin{array}{r}24 J u 190 \\
T-285 \\
\text { (ppb) }\end{array}$ & $\begin{array}{r}24 \mathrm{Jul} 90 \\
\mathrm{~T}-252 \\
\text { (ppb) }\end{array}$ & $\begin{array}{r}24 J u 190 \\
\text { T-284 } \\
\text { (ppb) }\end{array}$ & $\begin{array}{r}24 \mathrm{Ju} 190 \\
\mathrm{~T}-275 \\
\text { (ppb) }\end{array}$ & $\begin{array}{c}24 J u 190 \\
\text { T-279 } \\
\text { (ppb) }\end{array}$ \\
\hline Di-n-Octylphthalate & $N A$ & NA & $\mathrm{NA}$ & $N A$ & NA & $N A$ & $\mathrm{NA}$ \\
\hline Berzo(b) fluoranthene & NA & NA & NA & NA & NA & $\mathrm{NA}$ & NA \\
\hline Berro(k)fluoranthene & NA & NA & $N A$ & $\mathrm{NA}$ & $\mathrm{NA}$ & NA & NA \\
\hline Berzo(a)pyrene & NA & $\mathrm{NA}$ & $\mathrm{NA}$ & NA & NA & NA & NA \\
\hline Indeno $(1,2,3-\infty)$ pyrene & NA & NA & $\mathrm{NA}$ & NA & NA & NA & NA \\
\hline Diberz $(a, h)$ antrracene & NA & NA & $\mathrm{NA}$ & $N A$ & NA & NA & NA \\
\hline $\operatorname{Berzo}(\mathrm{g}, \mathrm{h}, \mathrm{i})$ perylene & NA & NA & $\mathrm{NA}$ & NA & NA & NA & NA \\
\hline Acrylanide & NA & NA & NA & NA & NA & NA & NA \\
\hline Silver & $N A$ & NA & NA & NA & $N A$ & $\mathrm{NA}$ & NA \\
\hline Aluminm & $N A$ & NA & NA & NA & NA & NA & NA \\
\hline Arsenic & $\mathrm{NA}$ & NA & $N A$ & NA & $N A$ & $N A$ & NA \\
\hline Boran & $\mathrm{NA}$ & NA & $N A$ & $N A$ & NA & NA & NA \\
\hline Barium & NA & NA & NA & NA & $\mathrm{NA}$ & $\mathrm{NA}$ & NA \\
\hline Beryllium & NA & NA & NA & NA & NA & NA & NA \\
\hline Calcium & $\mathrm{NA}$ & $\mathrm{NA}$ & NA & NA & NA & NA & NA \\
\hline Cadmium & $\mathbb{N A}$ & NA & $\mathrm{NA}$ & NA & $N A$ & NA & NA \\
\hline Cobalt & NA & NA & $\mathrm{NA}$ & NA & NA & NA & NA \\
\hline Arranium & NA & NA & NA & NA & NA & NA & NA \\
\hline Copper & NA & NA & NA & NA & NA & NA & NA \\
\hline Iran & NA & NA & NA & NA & NA & NA & NA \\
\hline Merany & $\mathrm{NA}$ & NA & NA & $N A$ & NA & NA & NA \\
\hline Potassium & NA & NA & NA & NA & NA & NA & NA \\
\hline Lithium & NA & NA & $\mathrm{NA}$ & NA & NA & NA & NA \\
\hline Magresium & NA & NA & NA & $\mathrm{NA}$ & NA & NA & NA \\
\hline Manganese & $N A$ & $\mathrm{NA}$ & NA & NA & NA & NA & NA \\
\hline Mblybodenm & NA & NA & NA & NA & NA & NA & NA \\
\hline Sodium & NA & NA & NA & NA & $N A$ & NA & NA \\
\hline Nickel & NA & $\mathrm{NA}$ & $\mathrm{NA}$ & NA & $N A$ & NA & NA \\
\hline Thasphonus & $\mathrm{NA}$ & $\mathrm{NA}$ & $N A$ & $N A$ & NA & NA & NA \\
\hline Lead & NA & NA & NA & NA & NA & NA & NA \\
\hline Antimany & $\mathrm{NA}$ & NA & $\mathrm{NA}$ & $\mathrm{NA}$ & NA & NA & NA \\
\hline Selenium & NA & NA & $N A$ & NA & NA & NA & NA \\
\hline Silioan & NA & NA & $\mathrm{NA}$ & NA & $\mathrm{NA}$ & NA & NA \\
\hline $\operatorname{Tin}$ & $\mathrm{NA}$ & NA & NA & NA & NA & NA & NA \\
\hline Strantium & $N A$ & NA & NA & $\mathrm{NA}$ & NA & NA & $\mathrm{NA}$ \\
\hline Titanium & $N A$ & NA & NA & NA & NA & NA & NA \\
\hline Vanadium & NA & NA & NA & NA & NA & $N A$ & NA \\
\hline Zinc & NA & NA & NA & $N A$ & NA & NA & NA \\
\hline Ziroanium & $N A$ & $N A$ & NA & NA & NA & NA & NA \\
\hline Bramide & NA & $\mathrm{NA}$ & NA & NA & NA & $N A$ & NA \\
\hline Chloride & NA & NA & $\mathrm{NA}$ & NA & NA & NA & NA \\
\hline Fluoride & $N A$ & NA & $\mathrm{NA}$ & NA & NA & NA & $\mathrm{NA}$ \\
\hline Nitrate & $\mathrm{NA}$ & NA & NA & NA & NA & NA & $\mathrm{NA}$ \\
\hline Phosphate & NA & NA & $N A$ & NA & NA & NA & NA \\
\hline Sulfate & NA & NA & NA & NA & NA & NA & $\mathrm{NA}$ \\
\hline Total Organic Camban & $\mathrm{NA}$ & NA & NA & NA & NMA & NA & NAS \\
\hline
\end{tabular}


Table D.4 (ontinuad)

Sampling Date:

Burial Trench Nunber:

24Ju190 24Ju190 24Ju190 24Ju190 24Ju190 24Ju190 24Ju190

Species/Chamical:

$\begin{array}{lllllll}\text { T-11 } & \text { T-288 } & \text { T-285 } & \text { T-252 } & \text { T-284 } & \text { T-275 } & \text { T-279 }\end{array}$

Carban-14

Cobalt- 60

Cesium-137

Gross Alpha

Gross Beta

Strantium-90

Tritium

Americium-241

arium-244

Plutanium-239

Uranium-232

Uranim-233

pH

Electrical Conductivity

$\frac{(\mathrm{ppb})}{(\mathrm{Bq} / \mathrm{L})} \frac{(\mathrm{ppb})}{(\mathrm{Bq} / \mathrm{L})} \frac{(\mathrm{ppb})}{(\mathrm{Bq} / \mathrm{L})} \frac{(\mathrm{ppb})}{(\mathrm{Bq} / \mathrm{L})} \frac{(\mathrm{ppb})}{(\mathrm{Bq} / \mathrm{L})} \frac{(\mathrm{ppb})}{(\mathrm{Bq} / \mathrm{L})} \frac{(\mathrm{ppb})}{(\mathrm{Bq} / \mathrm{L})}$

Dissolved Solids

Total Solids

Suspended Solids

Handhess

Alkalinity

\begin{tabular}{|c|c|c|c|c|c|}
\hline $\mathrm{NA}$ & $N A$ & NA & NA & NA & NA \\
\hline $\mathrm{NA}$ & NA & $N A$ & NA & NA & NA \\
\hline $\mathrm{NA}$ & NA & $N A$ & NA & NA & NA \\
\hline $\mathrm{NA}$ & NA & $N A$ & NA & NA & NA \\
\hline $\mathrm{NA}$ & NA & $N A$ & NA & NA & NA \\
\hline $\mathrm{NA}$ & NA & NA & NA & $\mathrm{NA}$ & NA \\
\hline $\mathrm{NA}$ & $N A$ & $\mathrm{NA}$ & NA & NA & NA \\
\hline NA & NA & $\mathrm{NA}$ & NA & NA & NA \\
\hline $\mathrm{NA}$ & NA & NA & NA & NA & NA \\
\hline $\mathrm{NA}$ & $\mathrm{NA}$ & $N$ & NA & NA & NA \\
\hline $\mathrm{NA}$ & NA & $\mathrm{NA}$ & NA & NA & NA \\
\hline $\mathrm{NA}$ & $N A$ & $\mathrm{NA}$ & NA & $\mathrm{NA}$ & NA \\
\hline 5.5 & 6.6 & 6.4 & 6.8 & 6.4 & 6.1 \\
\hline 1629 & 606 & 417 & 505 & 614 & 358 \\
\hline 1760 & 480 & 300 & 240 & 360 & 180 \\
\hline NA & NA & NA & NA & $N A$ & NA \\
\hline $\mathrm{NA}$ & NA & $N A$ & NA & NA & NA \\
\hline 700 & 268 & 204 & 224 & 394 & 204 \\
\hline 610 & 310 & 284 & 325 & 419 & 223 \\
\hline
\end{tabular}


Thable D.5. Chemical analyses of SHSA 6 burial trench leachates

\begin{tabular}{|c|c|c|c|c|c|c|c|}
\hline Sampling Date: & 0916089 & $09 \mathrm{Nbv} 69$ & O9Nov89 & $09 \mathrm{Nov} 69$ & 0916089 & 09 16u89 & OGApl89 \\
\hline Burial Trench Nuber: & $\mathrm{T}-13$ & $\mathrm{~T}-279$ & $\mathrm{~T}-288$ & $\mathrm{~T}-85$ & T-105 & T-318 & $\mathrm{T}-237$ \\
\hline Species/thamical: & (pab) & (ppb) & (ppb) & (ppb) & (ppb) & (ppb) & (ppb) \\
\hline Chlorcmethane & 2500 & $\varangle 000$ & 850 & 2500 & 40000 & $\triangle 1000$ & 40 \\
\hline Bromamethane & 2500 & $\triangleleft 000$ & $<50$ & 2500 & $\triangle 0000$ & $\triangle 000$ & $<0$ \\
\hline Vinyl chloride & 2500 & $\triangleleft 000$ & 850 & 8500 & $\triangle 0000$ & $\triangleleft 1000$ & 40 \\
\hline Chloroethane & 8500 & $\triangle 000$ & $<50$ & 2500 & $\triangleleft 0000$ & $\triangle 000$ & $\triangle 0$ \\
\hline Methylene ciloride & JB390 & JB107 & JB37 & JB280 & JB2900 & $\mathrm{JB120}$ & B7 \\
\hline Acetane & 2800 & 1200 & JB220 & 3100 & 12500 & 1200 & B51 \\
\hline Caban disulfide & $\triangle 300$ & $<00$ & $\triangle 30$ & $\triangle 300$ & 5000 & $<00$ & J0.7 \\
\hline 1,1-Dichloroethere & $\triangleleft 300$ & $\diamond 00$ & 430 & 4300 & $<000$ & $<00$ & $<$ \\
\hline 1,1-Dichlonoethane & $\triangleleft 300$ & $\$ 00$ & $<30$ & $\triangle 300$ & 5000 & $\measuredangle 00$ & $<$ \\
\hline 1,2-Dichlonoethene (Total) & $\triangle 300$ & $\$ 00$ & $\triangleleft 30$ & $\triangle 300$ & $<000$ & $<00$ & $<$ \\
\hline Chloroform & $\triangle 300$ & $<00$ & $\triangle 30$ & $\triangle 300$ & 5300 & $\$ 00$ & $\infty$ \\
\hline 1,2-Dichloroethane & $\triangle 300$ & $\$ 00$ & $<30$ & $\triangle 300$ & $<000$ & $\$ 00$ & 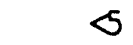 \\
\hline 2-Butanane & 2500 & $\triangle 000$ & $<50$ & 2500 & $\varangle 0000$ & $\varangle 000$ & B19 \\
\hline 1,1,1-Trichloroethane & $\triangle 300$ & $\diamond 00$ & $\triangle 30$ & $\triangle 300$ & $<000$ & $\diamond 00$ & $<$ \\
\hline Carban tetrachloride & $\triangle 300$ & $<00$ & 430 & $\triangleleft 300$ & $<000$ & $<00$ & 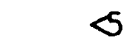 \\
\hline Viryl acetate & 2500 & $\triangle 000$ & $<50$ & 2500 & $\triangle 0000$ & $\varangle 000$ & $\triangle 0$ \\
\hline Bramodichlonamethane & $\triangle 300$ & $<00$ & 430 & $\triangle 300$ & $\$ 000$ & $<00$ & 5 \\
\hline 1,2-Dichlonopropane & $\triangleleft 300$ & $\$ 00$ & $\triangle 30$ & $\triangle 300$ & $<000$ & $\$ 00$ & $<$ \\
\hline cis-1,3-Dichloropropene & $\triangleleft 300$ & $\diamond 00$ & $<30$ & $\triangle 300$ & 5000 & $<00$ & $\sigma$ \\
\hline Trichloroethene & $\triangle 300$ & $<00$ & $<30$ & $\triangle 300$ & $<000$ & $\$ 00$ & $<$ \\
\hline Dibramochloramethane & $\triangleleft 300$ & $<00$ & $\triangle 30$ & $\triangle 300$ & $<000$ & $\$ 00$ & 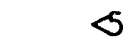 \\
\hline 1,1,2-Trichloroethane & $\triangle 300$ & $\$ 00$ & $\triangle 30$ & $\triangle 300$ & $<000$ & $\$ 00$ & $<$ \\
\hline Berzene & $\triangleleft 300$ & $\$ 00$ & $<30$ & $\triangle 300$ & $<000$ & $\$ 00$ & 28 \\
\hline trans-1,3-Dichloropropene & $\triangle 300$ & $\$ 00$ & $\triangle 30$ & $\triangle 300$ & $s 000$ & $\$ 00$ & $\infty$ \\
\hline Bramofonn & $<300$ & $\$ 00$ & 430 & $\triangle 300$ & $<000$ & 500 & 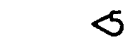 \\
\hline 4-Methyl-2-pentanane & 2500 & $\triangleleft 000$ & $<250$ & 2500 & $\triangle 0000$ & $\triangleleft 000$ & $<0$ \\
\hline 2-Hexamane & 8500 & $\varangle 000$ & $<50$ & 8500 & $<10000$ & $\varangle 000$ & $<0$ \\
\hline Tetrachlonøethene & $<300$ & $\ll 00$ & 430 & $\triangle 300$ & $<000$ & $\$ 00$ & $<$ \\
\hline 1,1,2,2-tetrachloncethane & $\triangleleft 300$ & $<00$ & 430 & $\triangleleft 300$ & $<000$ & $\$ 00$ & 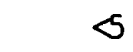 \\
\hline Toluene & 6600 & 9500 & 180 & 18000 & 74000 & 8400 & E2500 \\
\hline Chloroberzene & $\triangle 300$ & $\$ 00$ & $\triangle 30$ & $\triangle 300$ & $\$ 000$ & $\$ 00$ & $\diamond$ \\
\hline Ethylberzere & 3300 & $\$ 00$ & $<30$ & $\triangle 300$ & $<000$ & $<00$ & 190 \\
\hline Styrene & $\triangle 300$ & $<00$ & 430 & 4300 & $<000$ & $\$ 00$ & $\sigma$ \\
\hline Xylene (Total) & 28000 & 2500 & 420 & 12000 & 21000 & 9600 & E1700 \\
\hline Fhenol & 140 & $\varangle 30$ & $\triangle 3$ & Л6 & 760 & $\mathrm{~J} 36$ & $<1$ \\
\hline bis(2-Chloroethyl)ether & $\triangleleft 20$ & $<30$ & 43 & $\triangleleft 20$ & $\triangle 30$ & $<20$ & $\Delta 11$ \\
\hline 2-Chlorophenol & $\triangle 20$ & $<30$ & 43 & 420 & 430 & 420 & $\triangleleft 1$ \\
\hline 1,3-Dichlondberzene & $\triangleleft 20$ & $\triangleleft 30$ & $<3$ & $\triangleleft 20$ & $\triangle 30$ & $\triangleleft 20$ & $\triangleleft 1$ \\
\hline 1,4-Dichlonoberzene & $\triangleleft 20$ & $<30$ & $<3$ & $\triangleleft 20$ & $\triangle 30$ & $\triangle 20$ & $\triangleleft 1$ \\
\hline Benzyl alochol & $\triangle 20$ & $\triangleleft 30$ & 43 & $\triangleleft 20$ & $\triangleleft 30$ & $\triangle 20$ & $<1$ \\
\hline 1,2-Dichloraberzene & $\triangle 20$ & $<30$ & $<3$ & $\triangleleft 20$ & $\triangleleft 30$ & $\triangle 20$ & $\triangleleft 1$ \\
\hline 2-Methylyhenol & $\mathrm{J} 26$ & $\triangleleft 30$ & $<3$ & $\sqrt{2} 3$ & 200 & $<20$ & $\triangleleft 1$ \\
\hline bis(2-Chloroisopropyl)ether & $\triangleleft 20$ & $\triangleleft 30$ & $<3$ & $\triangle 20$ & $\triangleleft 30$ & $<20$ & $<1$ \\
\hline 4-Methylyhenol & 1800 & J69 & $<3$ & 500 & 4600 & 740 & $<1$ \\
\hline N-nitroso-di-n-propylamine & $\triangle 20$ & $\varangle 30$ & $<3$ & $\triangleleft 20$ & $\triangleleft 30$ & $\triangleleft 20$ & $\triangleleft 1$ \\
\hline Hexartilnmethane & $\Delta x$ & 430 & $<3$ & 420 & $\triangle 130$ & 420 & $\Delta 1$ \\
\hline
\end{tabular}


Table D.5 (ontinuod)

Sampling Date:

Burial Trench Number:

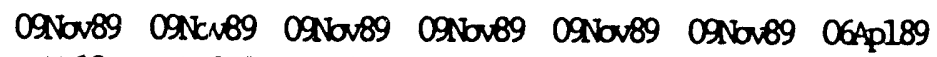

Species/Chemical:

Nitruberzene

Isophonane

2-Nitropheml

2,4-Dimethylphenol

Benzoic acid

bis(2-chloroethaxy)methane

2,4-Dichlorophenol

1,2,4-Trichloncbenzene

Nephthalene

4-chlonoaniline

Hexachlonobutadiene

4-Chlono-3-methylphemol

2-Methylnaphthalene

Hexachlomocyclopentadiene

2,4,6-Trichlonghemol

2,4,5-Trichlonophenol

2-Chlonanghthalene

2-Nitroaniline

Dimethylphthalate

Acenaphthylene

2,6-Dinitrotoluene

3-Nitroaniline

Acenaphthene

2,4-Dinitrophenol

4-Nitrophenol

Diberzofican

2,4-Dinitrotoluene

Diethylphthalate

4-Ohlonophenyl-phenylether

Fluonere

4-Nitroaniline

4,6-Dinitro-2-methylphenol

$\mathrm{N}$-nitrosotiphenylamine

4-bromophenyl-phenylether

Hexachlomoberzene

Pertachlorophenol

Fhenanthere

Anthracere

Di-n-butylphthalate

Fluosanthene

Pyrene

Butylberzylphthalate

3,3'-Dichlomberkidene

Berzo(a)anthracene

Onyysene

bis(2-Ethlyexyl)ththalate

\begin{tabular}{|c|c|c|c|c|c|c|}
\hline $\begin{array}{c}\mathrm{T}-13 \\
\text { (pab) }\end{array}$ & $\begin{array}{l}\text { r-279 } \\
\text { (ppb) }\end{array}$ & $\begin{array}{l}\text { T-288 } \\
\text { (ppb) }\end{array}$ & $\begin{array}{l}\text { T-85 } \\
\text { (pob) }\end{array}$ & $\begin{array}{l}\text { T-105 } \\
\text { (ppb) }\end{array}$ & $\begin{array}{l}T-318 \\
\text { (ppb) }\end{array}$ & $\begin{array}{l}\text { T-237 } \\
\text { (pob) }\end{array}$ \\
\hline 420 & $<130$ & 43 & 420 & 430 & 420 & 41 \\
\hline 420 & $<130$ & $<3$ & $\triangle 20$ & $<30$ & $\triangle 20$ & $\Delta 1$ \\
\hline$\triangle 20$ & $<130$ & 43 & $\triangle 20$ & 430 & 420 & $\Delta 1$ \\
\hline 420 & $<130$ & $<3$ & $<20$ & 160 & 380 & 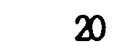 \\
\hline 610 & $<530$ & $\varangle 65$ & $\mathrm{~J} 250$ & $J 540$ & $\varangle 10$ & 87 \\
\hline 420 & 430 & $\triangle 3$ & $\triangle 20$ & $<30$ & 420 & $\Delta 1$ \\
\hline$\triangle 20$ & 130 & 43 & $\triangle 20$ & $<30$ & $\triangle 20$ & $<1$ \\
\hline 420 & 130 & 43 & $\triangle 20$ & 430 & 420 & $\Delta 1$ \\
\hline 3000 & 1000 & 18 & 2000 & 8800 & 1100 & 240 \\
\hline 420 & 430 & 43 & 420 & 430 & $<20$ & $\triangle 11$ \\
\hline 420 & $\triangle 130$ & $<3$ & $<20$ & $<30$ & $\triangle 20$ & $\triangle 1$ \\
\hline 420 & $\triangle 130$ & 43 & $\triangle 20$ & 430 & $<20$ & $<1$ \\
\hline$\triangle 20$ & $\triangleleft 30$ & $\triangle 3$ & $\triangle 20$ & $\triangle 30$ & $\triangle 20$ & 4 \\
\hline 420 & $<30$ & $\triangle 3$ & $\triangleleft 20$ & $\triangle 30$ & $\triangle 20$ & $<1$ \\
\hline$\triangle 20$ & $\varangle 30$ & 43 & $<20$ & $<30$ & $<20$ & $\triangleleft 1$ \\
\hline 610 & $\varangle 30$ & $\varangle 65$ & $\varangle 620$ & 630 & $\varangle 610$ & 57 \\
\hline 420 & $\triangleleft 30$ & $\triangle 3$ & $\triangle 20$ & $\triangle 30$ & $\triangle 20$ & $\Delta 1$ \\
\hline 810 & $\varangle 530$ & $\varangle 65$ & $\varangle 20$ & 630 & $\varangle 10$ & $<$ \\
\hline 420 & $\triangleleft 30$ & $\triangle 3$ & $\triangle 20$ & 430 & $<20$ & $\Delta 1$ \\
\hline 420 & $\triangleleft 30$ & $\triangle 3$ & $\triangleleft 20$ & $\triangle 30$ & $\triangle 20$ & $\triangleleft$ \\
\hline$\triangle 20$ & $<30$ & $<3$ & $\triangle 20$ & $<30$ & $\triangle 20$ & $<11$ \\
\hline 610 & $\varangle 630$ & $\varangle 65$ & $\varangle 620$ & 830 & $\varangle 10$ & 87 \\
\hline 420 & $\triangleleft 30$ & $\triangle 3$ & $\triangleleft 20$ & $\triangle 30$ & 420 & $\Delta 1$ \\
\hline$\varangle 10$ & $\varangle 630$ & 865 & $\varangle 620$ & $\varangle 30$ & $<610$ & $<7$ \\
\hline 810 & $\varangle 30$ & 865 & $\varangle 620$ & 630 & $\varangle 610$ & 5 \\
\hline$\triangle 20$ & 430 & $\triangle 3$ & $<20$ & 430 & 120 & $\Delta 1$ \\
\hline 420 & 430 & $<3$ & $\varangle 20$ & $<130$ & $\triangle 20$ & $<1$ \\
\hline$\triangle 20$ & 430 & $\Delta 3$ & $<20$ & $<30$ & $\triangle 20$ & $\Delta 1$ \\
\hline 420 & 130 & 43 & $<20$ & 430 & $\triangle 20$ & $\Delta 1$ \\
\hline 420 & $<30$ & $<3$ & $\triangle 20$ & $\triangleleft 30$ & $\triangle 20$ & $\triangleleft 1$ \\
\hline 610 & $\varangle 630$ & $\varangle 65$ & $\varangle 620$ & $\varangle 630$ & $\varangle 10$ & $<7$ \\
\hline$\varangle 10$ & $\varangle 630$ & $\varangle 65$ & $\varangle 620$ & 830 & $\varangle 610$ & $\Delta$ \\
\hline 420 & $<30$ & $<3$ & $\triangleleft 20$ & $<30$ & $<20$ & $<$ \\
\hline 420 & $\triangleleft 30$ & 43 & $\triangleleft 20$ & $\triangleleft 30$ & $<20$ & $<$ \\
\hline 420 & $\varangle 30$ & $<3$ & $\triangle 20$ & 430 & $\triangle 20$ & 4 \\
\hline$\varangle 610$ & $\varangle 630$ & $\varangle 65$ & $\varangle 620$ & $\varangle 30$ & $<610$ & $<$ \\
\hline$\varangle 20$ & 430 & $\triangle 3$ & $\triangle 20$ & $\triangle 30$ & 420 & 4 \\
\hline$\triangle 20$ & $\triangleleft 30$ & 43 & $\triangle 20$ & $<30$ & $\triangle 20$ & $\Delta 1$ \\
\hline 420 & $\triangleleft 30$ & $<3$ & $\triangle 20$ & 430 & $<20$ & 4 \\
\hline$\triangle 20$ & $\varangle 30$ & $\triangle 3$ & $\triangle 20$ & $<30$ & 420 & $\triangleleft$ \\
\hline$\triangle 20$ & $\triangle 30$ & 43 & $\triangleleft 20$ & 430 & $<20$ & $<$ \\
\hline $4 x$ & $\varangle 30$ & $\triangle 3$ & $\triangleleft 20$ & $\triangleleft 30$ & $\triangle 120$ & $<$ \\
\hline 240 & 250 & 26 & $<250$ & 250 & $<40$ & 2 \\
\hline 420 & $\varangle 30$ & $<3$ & $\triangleleft 20$ & $<30$ & $\triangle 20$ & 4 \\
\hline$\triangle 20$ & $<30$ & $<3$ & $\triangleleft 20$ & $\triangleleft 30$ & $\triangle 20$ & $\triangleleft$ \\
\hline$<20$ & 130 & $<13$ & $<20$ & $<130$ & 420 & $<$ \\
\hline
\end{tabular}

D-19 
Table D.5 (continued)

\begin{tabular}{|c|c|c|c|c|c|c|c|}
\hline Sampling Date: & ognov89 & ogNov89 & 09 16v89 & o9Nov89 & 09 Nav89 & ogsov89 & O6Ap189 \\
\hline $\begin{array}{l}\text { Arial Trench Nuber: } \\
\text { Species themical. }\end{array}$ & T-13 & T-279 & $\mathrm{T}-288$ & T-85 & T-105 & T- 318 & $\mathrm{~T}-237$ \\
\hline$\frac{\text { Specles /nemical: }}{\text { Di-n-Octyluhthalate }}$ & $\frac{(\mathrm{ppb})}{\Delta x}$ & & & & & (ppb) & (ppb) \\
\hline & 420 & $\triangle 30$ & $<3$ & $\varangle 20$ & $\triangleleft 30$ & 420 & $\triangleleft 1$ \\
\hline Benzo(b)fluoranthene & $\triangle 20$ & $\triangleleft 30$ & $<3$ & $<20$ & 430 & $<20$ & $\Delta 1$ \\
\hline Benzo(k)flumanthere & $\triangle 20$ & $\triangle 30$ & $\varangle 3$ & $<20$ & 430 & $<20$ & $ه 1$ \\
\hline Lenzo(a)pyrere & $\triangle 20$ & $\triangleleft 30$ & $\varangle 3$ & $<120$ & $\triangle 30$ & $\triangle 20$ & $\varangle 1$ \\
\hline Indeno $(1,2,3-\infty)$ pyrene & 420 & $\triangleleft 30$ & $\triangleleft 3$ & $<20$ & 430 & 420 & $ه 1$ \\
\hline $\operatorname{Diberz}(\mathrm{a}, \mathrm{h})$ anthracene & $\triangle 20$ & $\triangleleft 30$ & $\varangle 3$ & $\varangle 20$ & $\triangleleft 30$ & $\triangle 20$ & $\triangleleft 1$ \\
\hline $\operatorname{Benzo}(g, h, 1)$ perylene & 420 & $\triangleleft 30$ & $<3$ & $\triangleleft 20$ & $\triangle 30$ & $\Delta 20$ & $\triangleleft 1$ \\
\hline Acrylamide & $\mathrm{NA}$ & NA & NA & NA & NA & NA & 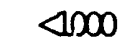 \\
\hline Silver & NA & NA & NA & NA & NA & $\mathrm{NA}$ & 15 \\
\hline Aluminm & NA & NA & NA & NA & NA & $\mathrm{NA}$ & 290 \\
\hline Arsenic & NA & NA & NA & NA & NA & NA & 69 \\
\hline Boman & NA & NA & NA & NA & NA & NA & $<0$ \\
\hline Barium & NA & $\mathrm{NA}$ & $\mathbb{N A}$ & $\mathrm{NA}$ & $\mathrm{N}_{1}$ & NA & 360 \\
\hline Beryllium & $\mathrm{NA}$ & NA & NA & NA & NA & NA & $\varangle 0.4$ \\
\hline Calcium & NA & NA & NA & NA & NA & NA & 130000 \\
\hline Cadmium & NA & $\mathrm{NA}$ & $\mathrm{NA}$ & NA & NA & NA & $<$ \\
\hline Cobalt & NA & NA & $\mathbb{N A}$ & NA & NA & NA & 31 \\
\hline Arranium & $\mathbb{N A}$ & NA & $\mathrm{NA}$ & NA & NA & NA & 12 \\
\hline Copper & $\mathrm{NA}$ & NA & NA & NA & NA & NA & $<10$ \\
\hline Iran & $\mathrm{NA}$ & NA & NA & NA & NA & NA & 11000 \\
\hline Merany & NA & NA & NA & NA & NA & NA & 0.3 \\
\hline Botassium & NA & NA & NA & NA & NA & NA & 5700 \\
\hline Lithium & $N A$ & NA & NA & NA & NA & NA & 45000 \\
\hline Magnesium & NA & NA & $\mathrm{NA}$ & NA & NA & NA & 20000 \\
\hline Manganese & NA & NA & NA & NA & NA & NA & 13000 \\
\hline Molybodenum & $\mathrm{NA}$ & NA & NA & NA & NA & NA & $\$ 10$ \\
\hline Sodium & $\mathrm{NA}$ & $N A$ & $\mathrm{NA}$ & $\mathrm{NA}$ & $N A$ & NA & 8000 \\
\hline Nickel & NA & $\mathrm{NA}$ & $\mathrm{NA}$ & NA & NA & $\mathrm{NA}$ & 6 \\
\hline Phosphans & NA & $N A$ & NA & NA & NA & NA & $<00$ \\
\hline Lead & NA & NA & NA & NA & NA & NA & $<0$ \\
\hline Antimany & NA & NA & NA & NA & NA & NA & 40 \\
\hline Selenium & NA & NA & NA & NA & NA & NA & $<80$ \\
\hline Sillioan & $N A$ & NA & NA & NA & NA & NA & 2200 \\
\hline Tin & $\mathrm{NA}$ & NA & $\mathrm{NA}$ & $\mathrm{NA}$ & NA & NA & $<0$ \\
\hline Strantium & $N A$ & NA & $\mathrm{NA}$ & NA & NA & NA & 170 \\
\hline Titanium & $N A$ & NA & NA & NA & NA & NA & $\infty$ \\
\hline Vanadium & $\mathrm{NA}$ & NA & NA & NA & NA & $\mathrm{NA}$ & $\varangle_{4}$ \\
\hline Zinc & NA & NA & $N A$ & NA & $\mathrm{NA}$ & NA & 200 \\
\hline Ziroanium & NA & NA & NA & NA & NA & NA & $\infty 0$ \\
\hline Bromide & NA & NA & $\mathrm{NA}$ & $\mathrm{NA}$ & NA & $\mathrm{NA}$ & 170 \\
\hline Chloride & $\mathrm{NA}$ & $\mathrm{NA}$ & $\mathrm{NA}$ & NA & $N A$ & $\mathrm{NA}$ & 13600 \\
\hline Fluoride & $\mathrm{NA}$ & $\mathrm{NA}$ & $\mathrm{NA}$ & $N A$ & NA & $\mathrm{NA}$ & 7230 \\
\hline Nitrate & $N A$ & NA & $N A$ & NA & NA & $\mathrm{NA}$ & 100 \\
\hline Hosphate & $N A$ & NA & NA & NA & NA & NA & $<0$ \\
\hline Sulfate & NA & NA & $\mathrm{NA}$ & NA & NA & NA & 280 \\
\hline Total Organic Carton & NA & NMA & Na & Nֵַ & Na & NA & 140000 \\
\hline
\end{tabular}


Table D.5 (ontinued)

Sampling Date:

Burial Trench Number:

O9Nov89 09Nov69 09Nov89 O9Nbv89 O9Nov89 O9N6v89 064p189

Species Themical:

Canban-14

Cobalt- 60

Cesium-137

Gross Alpha

Gross Beta

Strantium-90

Tritium

Americium-241

Arium-244

Plutanium-239

Uranium-232

Uranium-233

pH

Electrical Conductivity

Dissolved Solids

Total Solids

Suspended Solids

Handhess

Alkalinity

$\begin{array}{lllllll}\text { T-13 } & \text { T-279 } & \text { T-288 } & \text { T-85 } & \text { T-105 } & \text { T-318 } & \text { T-237 }\end{array}$

(ppb) (ppb) (ppb) (ppb) (ppb) (ppb) (ppb)

$\begin{array}{llllll}(\mathrm{Bq} / \mathrm{L}) & (\mathrm{Bq} / \mathrm{L}) \quad(\mathrm{Bq} / \mathrm{L}) \quad(\mathrm{Bq} / \mathrm{L}) \quad(\mathrm{Bq} / \mathrm{L}) \quad(\mathrm{Bq} / \mathrm{L}) \quad(\mathrm{Bq} / \mathrm{L})\end{array}$

NA NA NA NA NA NA

NA NA NA NA NA NA 8

NA NA NA NA NA NA 2.4

NA NA NA NA $\quad$ NA $\quad$ NA 8.4

NA NA NA NA NA NA 12

NA NA NA NA $N A \quad$ NA 0.14

NA NA NA NA NA NA 2700

NA NA NA NA NA NA NA

NA NA NA NA NA NA NA

NA NA NA NA NA NA NA

NA NA NA NA NA NA NA

NA NA NA NA NA NA NA

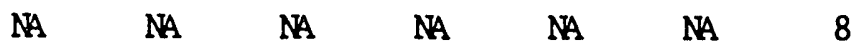

NA NA NA NA NA NA 554

NA NA NA NA NA NA 600

NA NA NA NA NA NA 840

NA NA NA NA NA NA 240

NA NA NA NA NA NA 372

NA NA NA NA NA NA 330 
Table D.6. Chemical analyses of SHSA 6 burial trench leachates

\begin{tabular}{|c|c|c|c|c|c|c|c|}
\hline Sampling Date: & 06Ap189 & 06Ap189 & $104 p 189$ & 06Ap189 & 06Ap189 & 064p189 & 06Ap189 \\
\hline Burial Trench Nuber: & $\mathrm{T}-112$ & T-101 & T-180 & $\mathrm{T}-318$ & $\mathrm{~T}-329$ & $\mathrm{~T}-82$ & $\mathrm{~T}-105$ \\
\hline Species/Chemical: & (ppb) & $(\mathrm{ppb})$ & (ppb) & (ppb) & $(\mathrm{ppb})$ & (ppb) & (pp) \\
\hline Chloramethane & $<0$ & $\triangle 0$ & $<0$ & $<0$ & $<10$ & $\triangle 0$ & $<0$ \\
\hline Bromamethane & $<0$ & $\triangle 0$ & $\Delta 0$ & $\triangle 0$ & $\Delta 0$ & $<0$ & $<10$ \\
\hline Vingl Chloride & $<0$ & $\triangle 0$ & $<0$ & $\triangleleft 0$ & $<0$ & $<0$ & $<0$ \\
\hline Chloroethane & 40 & $\triangleleft 10$ & $<0$ & $<0$ & $<10$ & $\triangleleft 0$ & $<10$ \\
\hline Methylene chloride & B8 & B5 & JB5 & $\mathrm{B} 19$ & JB4 & B19 & $B 45$ \\
\hline Acetane & BE 3600 & B55 & $\mathrm{B} 20$ & B130 & $B 64$ & BE6600 & BE3200 \\
\hline Camban disulfide & $\diamond$ & $\sigma$ & $\delta$ & $\sigma$ & 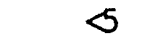 & $\delta$ & 50.6 \\
\hline 1,1-Dichlonoethene & $<$ & $\sigma$ & 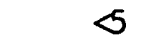 & 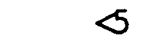 & $<$ & $<$ & $<$ \\
\hline 1,1-Didhlonoethame & 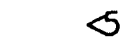 & 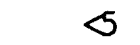 & $<$ & $<$ & $<$ & $\diamond$ & $<$ \\
\hline 1,2-Dichloroethene (Total) & $\diamond$ & 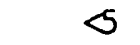 & $\diamond$ & $<$ & $\sigma$ & 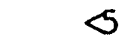 & $<$ \\
\hline Chloroform & $\Omega$ & 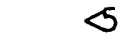 & 14 & $\Omega$ & $<$ & 8 & E560 \\
\hline 1,2-Dichloroethare & 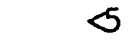 & 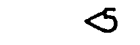 & 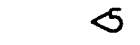 & $<$ & $<$ & 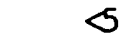 & 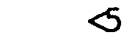 \\
\hline 2-Butanare & B95 & $\triangleleft 0$ & $\triangle 0$ & $\triangleleft 0$ & $\mathrm{~B} 20$ & $\triangle 0$ & B18 \\
\hline 1,1,1-Trichlonoethane & 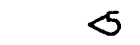 & $<$ & 5 & $<$ & $<$ & 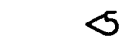 & $<$ \\
\hline Canban tetrachloride & 5 & 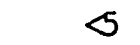 & $<$ & $<$ & 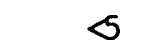 & $<$ & $\diamond$ \\
\hline Vinyl acetate & $<0$ & $<0$ & $<0$ & $\triangleleft 0$ & $<0$ & $\triangle 0$ & $\varangle 0$ \\
\hline Bromodichlorcmethane & 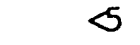 & $\diamond$ & $<$ & $<$ & $<$ & 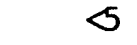 & 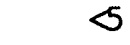 \\
\hline 1,2-Dichloropropane & 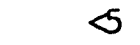 & 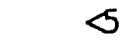 & 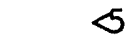 & $<$ & $<$ & 5 & $<$ \\
\hline cis-1,3-Dichlonopropene & 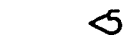 & 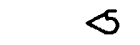 & 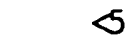 & $<$ & $<$ & $<$ & $<$ \\
\hline Trichloroethene & $\zeta$ & 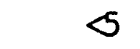 & $<$ & $<$ & 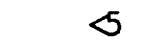 & $<$ & $<$ \\
\hline Dibromochloramethane & 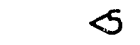 & 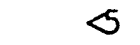 & $<$ & $<$ & $<$ & 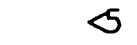 & 5 \\
\hline 1,1,2-Trichloroethane & 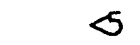 & $\sigma$ & $<$ & 5 & $\sigma$ & 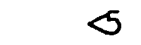 & $<$ \\
\hline Benzere & $\Omega$ & J0.6 & il & $\mathrm{J} 2$ & 50.8 & 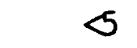 & 12 \\
\hline trans-1,3-Dichloropropene & 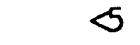 & 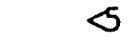 & 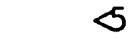 & $<$ & $\sigma$ & $\sigma$ & $\diamond$ \\
\hline Bramaform & 5 & $\triangleleft$ & 5 & $<$ & $<$ & 5 & $<$ \\
\hline 4-Methyl-2-pentanare & $\sqrt{3}$ & $\varangle 0$ & $\triangle 0$ & $<0$ & $<10$ & $<0$ & $<0$ \\
\hline 2-Hexanane & $<0$ & $<0$ & $\triangleleft 0$ & $\triangleleft 0$ & $\triangleleft 0$ & $<10$ & $<10$ \\
\hline Tetrachloroethene & $<$ & 5 & $<$ & $<$ & $\varsigma$ & 5 & 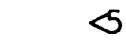 \\
\hline 1,1,2,2-tetrachlonoethane & 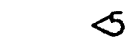 & 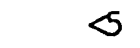 & 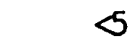 & $<$ & 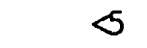 & 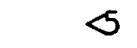 & $<$ \\
\hline Toluene & E260 & 83 & E5000 & E2500 & $\mathrm{E} 670$ & 18 & E3500 \\
\hline Chloroberzene & 5 & $<$ & $\sigma$ & $\sigma$ & $\sigma$ & 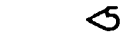 & $\sigma$ \\
\hline Ethylbenzene & 65 & $<$ & 69 & 140 & 41 & $<$ & 130 \\
\hline Styrene & $<$ & $\diamond$ & 23 & $<$ & 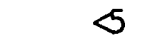 & $\sigma$ & 23 \\
\hline Xylene (Total) & $E+\infty 0$ & 31 & E730 & $E 3400$ & E510 & $\infty$ & $E 3000$ \\
\hline Phenol & $\triangleleft 1$ & $\triangleleft 2$ & $<2$ & $\triangleleft 2$ & 40 & $\triangle 2$ & $<1$ \\
\hline bis(2-Chlonoethyl)ether & $ه 1$ & 42 & $\triangleleft 2$ & $\varangle 2$ & $\varangle 2$ & $<2$ & $<1$ \\
\hline 2-Chlorophenol & $\triangleleft 1$ & $\measuredangle 2$ & $<2$ & $\triangleleft 2$ & $\varangle 2$ & $\triangle 2$ & $\triangleleft 1$ \\
\hline 1,3-Dichlorobenzene & $\triangleleft 1$ & $\varangle 2$ & $\varangle 2$ & $\varangle 2$ & $<2$ & $<2$ & $<1$ \\
\hline 1,4-Dichloroberzene & $\triangleleft 1$ & $<2$ & $<2$ & $\triangleleft 2$ & $<2$ & $\triangleleft 2$ & $\Delta 1$ \\
\hline Benzyl alochol & $\triangle 11$ & $<2$ & $\varangle 2$ & $\varangle 2$ & $<2$ & $\triangleleft 2$ & 41 \\
\hline 1,2-Dichlonoberzene & $\triangleleft 1$ & $\varangle 2$ & $\varangle 2$ & 42 & $<2$ & $\triangleleft 2$ & $<1$ \\
\hline 2-Methylphenol & $\triangle 1$ & $\varangle 2$ & 15 & $<2$ & $<2$ & $<2$ & $<1$ \\
\hline bis(2-Chloroisopropyl)ether & $\triangleleft$ & $\triangle 2$ & 42 & $\varangle 2$ & $\varangle 2$ & $<2$ & $\triangleleft 1$ \\
\hline 4-Methylphenol & $\Delta 1$ & $\triangle 12$ & 16 & $\varangle 2$ & $\mathrm{E} 600$ & $\varangle 2$ & E1200 \\
\hline N-nitroso-di-n-propylamine & $\triangleleft 1$ & $<12$ & $<2$ & $\varangle 2$ & $\varangle 2$ & $<2$ & $<1$ \\
\hline Hexachloroethane & $\triangleleft 1$ & $<2$ & $<2$ & $<2$ & $<2$ & $\varangle 2$ & $<1$ \\
\hline
\end{tabular}


Table D.6 (continued)

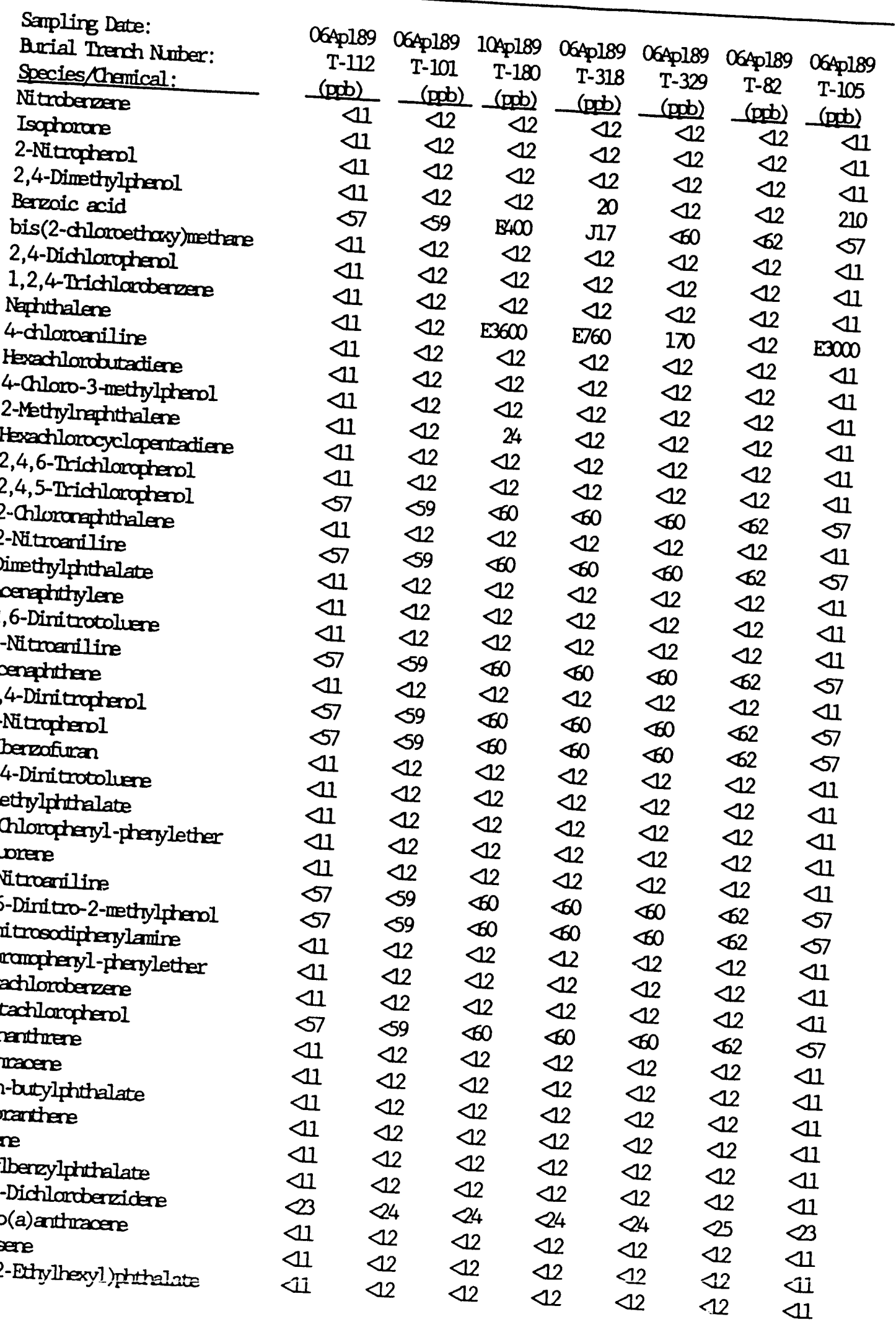


Table D.6 (oatinued)

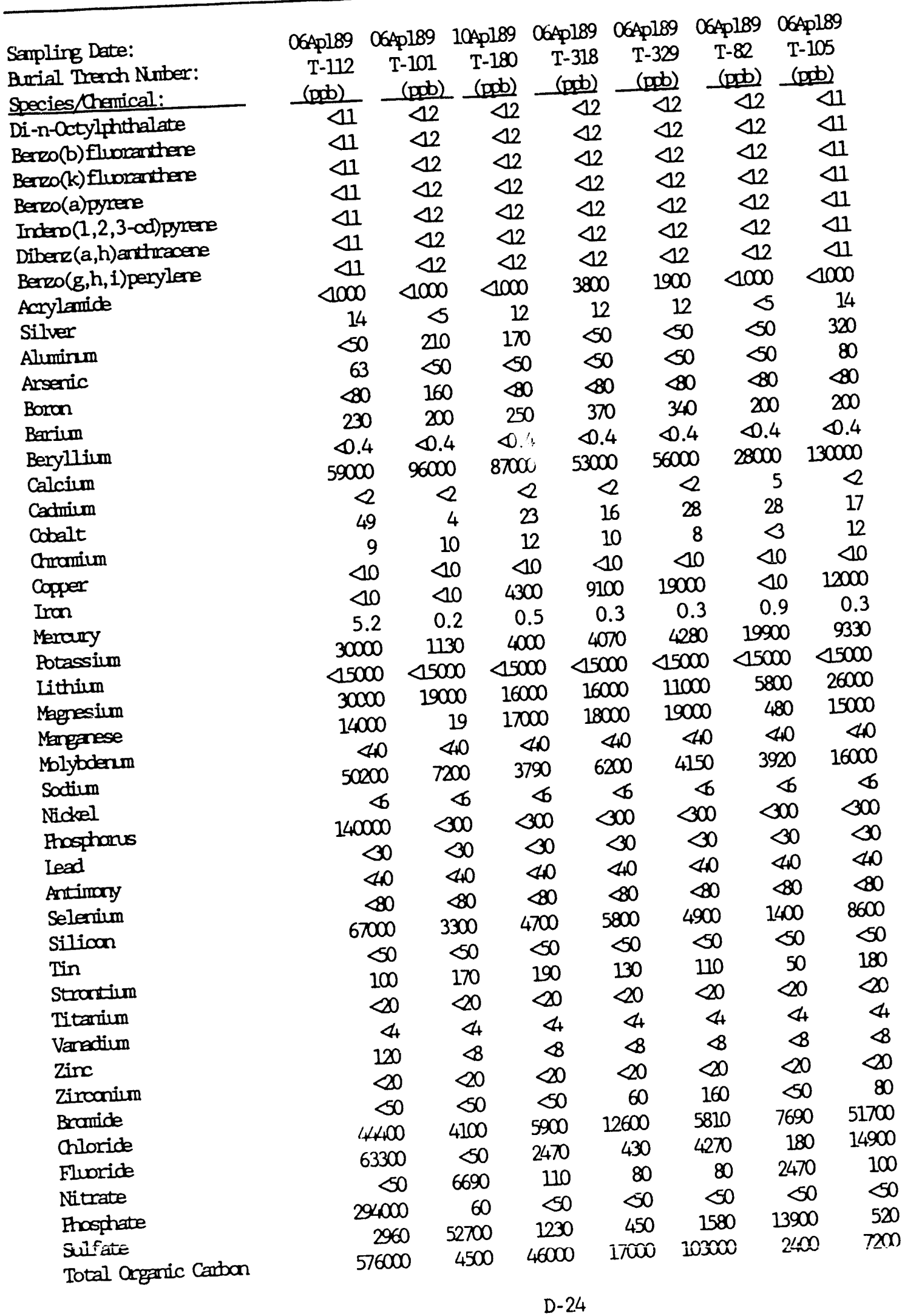


Table D.6 (ountinued)

Sampling Date:

Burial Trench Nuber:

Species/Chemical:

Carban-14

Cobalt -60

Cesium-137

Gross Alpha

Gross Beta

Strantium-90

Tritium

Americium-241

Arrium-244

Plutanium-239

Uranium-232

Uranium-233

HH

Electrical Conductivity

Dissolved Solids

Total Solids

Suspended Solids

Handess

Alkalinity

\begin{tabular}{|c|c|c|c|c|c|c|}
\hline $\begin{array}{c}\text { 06Ap189 } \\
\mathrm{T}-112 \\
\text { (pab) } \\
\end{array}$ & $\begin{array}{r}06 \mathrm{pp} 189 \\
\mathrm{~T}-101 \\
\text { (pob) }\end{array}$ & $\begin{array}{r}104 \mathrm{pl} 189 \\
\mathrm{~T}-180 \\
\text { (pab) }\end{array}$ & $\begin{array}{r}\text { 06Ap189 } \\
\mathrm{T}-318 \\
\text { (ppb) }\end{array}$ & $\begin{array}{r}\text { 06Ap189 } \\
\text { T-329 } \\
\text { (pob) }\end{array}$ & $\begin{array}{c}06 \text { pl89 } \\
\text { T-82 } \\
\text { (pdb) }\end{array}$ & $\begin{array}{c}06 \text { Ap189 } \\
\text { T-105 } \\
\text { (pd) }\end{array}$ \\
\hline$(\mathrm{Bg} / \mathrm{L})$ & $(\mathrm{Bq} / \mathrm{L})$ & $(\mathrm{Bg} / \mathrm{L})$ & $(\mathrm{Bg} / \mathrm{L})$ & $(\mathrm{Bg} / \mathrm{L})$ & $(\mathrm{Bq} / \mathrm{L})$ & $(\mathrm{Bq} / \mathrm{L})$ \\
\hline$<0$ & 1 & 2 & 12 & 12 & $<0$ & 17 \\
\hline$<$ & $<$ & 2 & $<$ & $<$ & $<$ & 2 \\
\hline 27 & 2 & 3.5 & 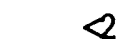 & $<$ & 12 & 4.7 \\
\hline$\varangle 0.4$ & $\$ 0.6$ & $\$ .4$ & 0.8 & $\$ .4$ & $\$ .4$ & $\$ .4$ \\
\hline 55 & 2.5 & 15 & 4.6 & 2.7 & 1100 & 21 \\
\hline 0.77 & 0.41 & 1.5 & $0.0_{4}$ & 0.80 & 660 & 0.83 \\
\hline 2700 & 150 & 470 & 1500 & 1200 & 32 & 1800 \\
\hline$N A$ & NA & NA & $N A$ & $\mathrm{NA}$ & NA & $N A$ \\
\hline NA & NA & $N A$ & NA & NA & NA & $\mathrm{NA}$ \\
\hline NA & NA & NA & NA & NA & NA & NA \\
\hline $\mathrm{NA}$ & NA & $\mathrm{NA}$ & $N A$ & NA & NA & NA \\
\hline$N A$ & NA & NA & NA & NA & NA & NA \\
\hline 7.6 & 8 & 7.85 & 7.9 & 7.65 & 7.5 & 6.9 \\
\hline 4620 & 356 & 369 & 335 & 323 & 160 & 774 \\
\hline 780 & 320 & 300 & 260 & 300 & 120 & 880 \\
\hline 1980 & 1240 & 380 & 400 & 2040 & 2400 & 1060 \\
\hline 1200 & 920 & 80 & 140 & 1740 & 2280 & 180 \\
\hline 168 & 290 & 274 & 208 & 184 & 96 & 400 \\
\hline 2002 & 231 & 277 & 236 & 186 & 79 & 291 \\
\hline
\end{tabular}


Table D.7. Chemical amalyses of SUSA 6 burial trench leachates

\begin{tabular}{|c|c|c|c|c|c|c|c|}
\hline Sampling Date: & O6Ap189 & 05Ap189 & 05Ap189 & $10 \mathrm{pp} 189$ & 10Apl89 & $29 \mathrm{Man} 89$ & $29 \mathrm{Mar} 89$ \\
\hline Burial Trench Numer: & $\mathrm{T}-85$ & $\mathrm{~T}-92-2$ & $\mathrm{~T}-92-1$ & $\mathrm{~T}-63$ & $T-44$ & T-5 & T-9 \\
\hline Species/Chemical: & & (ppb) & (ppb) & (ppo) & (ppb) & (ppb) & (ppb) \\
\hline Chloramethane & $<0$ & 40 & 40 & $\varangle 0$ & 50 & $\mathrm{NA}$ & NA \\
\hline Bromomethane & $\diamond 0$ & 40 & $\triangleleft 0$ & 40 & $<0$ & NA & NA \\
\hline Vinyl chloride & $<0$ & $<10$ & $\triangle 0$ & $\varangle 0$ & $<0$ & NA & NA \\
\hline Chloroethane & $<0$ & $<0$ & 40 & 40 & $<0$ & NA & NA \\
\hline Methylene chloride & JB12 & B5 & JB5 & $\mathrm{JB}_{4}$ & $\mathrm{JB} 12$ & NA & NA \\
\hline Acetane & BE 8300 & $B E 5400$ & B130 & B16 & B1,00 & NA & NA \\
\hline Canban disulfide & $\infty 5$ & $<$ & $<$ & $\diamond$ & $<5$ & NA & NA \\
\hline 1,1-Dichlonoethene & $<5$ & 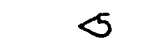 & 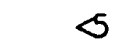 & 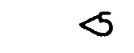 & 25 & NA & NA \\
\hline 1,1-Dichloroethane & 85 & $<$ & $\diamond$ & $\checkmark$ & $<5$ & NA & NA \\
\hline 1,2-Dichlonoethene (Total) & $\infty 5$ & 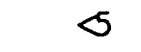 & 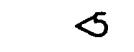 & $\sigma$ & 25 & $\mathrm{NA}$ & NA \\
\hline Chloroform & $\mathrm{J} 16$ & 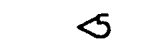 & $\mathrm{J} 3$ & J0.6 & $J_{4}$ & $\mathrm{NA}$ & NA \\
\hline 1,2-Dichloroethane & $<5$ & 5 & 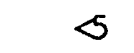 & $\diamond$ & 85 & $\mathrm{NA}$ & NA \\
\hline 2-Butanane & $<0$ & $\triangleleft 0$ & J0.5 & 40 & $<0$ & NA & NA \\
\hline 1,1,1-Trichlonoethane & $\infty 5$ & $<$ & $<$ & 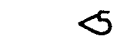 & $<5$ & NA & NA \\
\hline Carban tetrachloride & 25 & $\sigma$ & $\diamond$ & $\checkmark$ & $<5$ & NA & NA \\
\hline Vinyl acetate & $<0$ & $<0$ & $\triangleleft 0$ & $\triangleleft 0$ & $\diamond 0$ & NA & NA \\
\hline Bromodichloromethane & $<5$ & 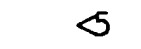 & 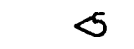 & 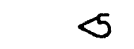 & 85 & $\mathrm{NA}$ & NA \\
\hline 1,2-Dichloropropane & $<25$ & $<$ & $<$ & 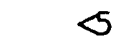 & $<5$ & NA & NA \\
\hline cis-1,3-Dichloropropene & $<5$ & $<$ & $<$ & 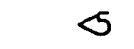 & 85 & NA & NA \\
\hline Trichloroethene & B36 & JB5 & $\mathrm{JB}_{4}$ & $J_{B 4}$ & JB16 & NA & NA \\
\hline Dibnumochloramethane & 25 & $<$ & $<$ & 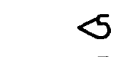 & 85 & NA & $N A$ \\
\hline 1,1,2-Trichlarcethane & 85 & 5 & $\delta$ & 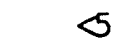 & 25 & NA & NA \\
\hline Berzene & $\infty 5$ & 5 & $\delta$ & 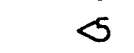 & 85 & $\mathrm{NA}$ & NA \\
\hline trans-1,3-Dichlonopropene & $<5$ & $<$ & $<$ & $<$ & $<5$ & NA & NA \\
\hline Bramoform & $\infty 5$ & $<$ & 5 & 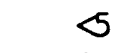 & 85 & NA & NA \\
\hline 4-Methyl-2-pentamane & $<0$ & $\triangle 0$ & $<0$ & $\triangleleft 0$ & $<0$ & NA & NA \\
\hline 2-Hexamane & $\diamond 0$ & 40 & $<10$ & 40 & $\measuredangle 0$ & NA & NA \\
\hline Tetrachlorøethene & $<5$ & 5 & $<$ & $\triangleleft$ & 85 & NA & NA \\
\hline 1,1,2,2-tetrachloroethane & $<5$ & 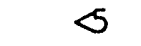 & $<$ & 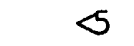 & 25 & $\mathrm{NA}$ & NA \\
\hline Toluene & BE 3200 & B7 & B41 & B75 & $\mathrm{B} 220$ & $\mathrm{NA}$ & NA \\
\hline Chlonobervene & $<5$ & $<$ & $<$ & 5 & 85 & NA & NA \\
\hline Erhylbenzene & 80 & 5 & $<$ & 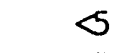 & $<5$ & NA & NA \\
\hline Styrene & 25 & $<$ & $<$ & 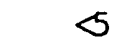 & $<5$ & NA & NA \\
\hline Xylene (Total) & E1900 & $<$ & 19 & 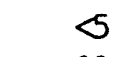 & 85 & NA & NA \\
\hline Fenol & 19 & $<2$ & $<2$ & $\triangleleft 0$ & $\triangleleft 0$ & NA & NA \\
\hline bis(2-Chloroethyl)ether & $ه 1$ & $\varangle 2$ & $<2$ & $\triangle 0$ & $\triangle 0$ & $\mathrm{NA}$ & NA \\
\hline 2-Chlonophenol & 41 & $<12$ & $<2$ & $\triangleleft 0$ & $\triangleleft 0$ & NA & NA \\
\hline 1,3-Dictilonoberzene & $\triangle 1$ & $\varangle 2$ & $<2$ & 40 & $<10$ & NA & NA \\
\hline 1,4-Dichloroberzene & $\triangleleft 1$ & 42 & 42 & 40 & $<0$ & NA & NA \\
\hline Berzyl alochol & $\triangleleft 1$ & $\triangle 2$ & $\varangle 2$ & 40 & 40 & NA & NA \\
\hline 1,2-Dichlonobenzene & $<1$ & $<2$ & $<2$ & $\triangleleft 0$ & $<0$ & NA & NA \\
\hline 2-Methylyhenol & $\triangleleft 1$ & $\varangle 2$ & $\varangle 2$ & $\triangleleft 10$ & $<0$ & NA & NA \\
\hline bis(2-Chloroisopropyl)ether & $\triangleleft 1$ & $\varangle 2$ & $<2$ & $\triangleleft 0$ & $<0$ & NA & NA \\
\hline 4-Methylphenol & 41 & $\varangle 2$ & $\varangle 2$ & $\triangleleft 0$ & $\varangle 0$ & NA & NA \\
\hline N-nitroso-di-n-propylamine & $\Delta 1$ & $<12$ & $<2$ & $\triangleleft 0$ & $\triangleleft 0$ & NA & NA \\
\hline Hexachioroethane & نَت & $<2$ & $<2$ & $<10$ & $<0$ & NA & NA \\
\hline
\end{tabular}


Table D.7 (continued)

\begin{tabular}{|c|c|c|c|c|c|c|c|}
\hline $\begin{array}{l}\text { Sampling Date: } \\
\text { Burial Trench Number: } \\
\text { Species/Chemical: }\end{array}$ & $\begin{array}{l}\text { 06Ap189 } \\
\text { T-85 } \\
\text { (ppb) } \\
\end{array}$ & $\begin{array}{r}\text { 05Ap } 189 \\
\mathrm{~T}-92-2 \\
\quad \text { (ppb) } \\
\end{array}$ & $\begin{array}{r}\text { 05Ap189 } \\
\text { T- } 92-1 \\
\text { (ppb) }\end{array}$ & $\begin{array}{l}10 \mathrm{pp} 189 \\
\mathrm{~T}-63 \\
\text { (ppb) }\end{array}$ & $\begin{array}{c}10 \mathrm{pp} 189 \\
\mathrm{~T}-44 \\
\text { (ppb) }\end{array}$ & $\begin{array}{l}29 \mathrm{mar} 89 \\
\mathrm{~T}-5 \\
\text { (ppb) }\end{array}$ & $\begin{array}{l}29 \mathrm{Mar} 89 \\
\mathrm{~T}-9 \\
\text { (pp) }\end{array}$ \\
\hline Nitroberzene & $<1$ & 42 & 42 & 40 & $<10$ & $\mathrm{NA}$ & $\mathrm{NA}$ \\
\hline Isqphonane & $\triangleleft 1$ & $\varangle 2$ & $\triangleleft 2$ & $<0$ & $\triangleleft 0$ & $N_{1}$ & NA \\
\hline 2-Nitrophenol & $\triangleleft 1$ & $\varangle 2$ & $\varangle 2$ & $\triangle 0$ & $\triangleleft 0$ & NA & NA \\
\hline 2,4-Dimethylphenol & $<1$ & $\triangleleft 2$ & $\triangleleft 2$ & $\triangleleft 0$ & $<10$ & $\mathrm{NA}$ & NA \\
\hline Benzoic acid & 57 & $\measuredangle 8$ & $\varangle 60$ & $\$ 0$ & $\diamond 0$ & $\mathrm{NA}$ & $\mathrm{NA}$ \\
\hline bis(2-chlonoethaxy)methane & $\triangleleft 1$ & 42 & $\varangle 2$ & 40 & 40 & $\mathbb{N A}$ & NA \\
\hline 2,4-Dichlonophenol & 41 & $<12$ & $\triangleleft 2$ & $\triangleleft 0$ & $\triangle 0$ & NA & NA \\
\hline 1,2,4-Trichlambenzene & $\varangle 1$ & $\varangle 2$ & $\varangle 2$ & $\triangleleft 0$ & $<0$ & NA & NA \\
\hline Nephthalene & E830 & $\varangle 2$ & $\varangle 2$ & $<0$ & 270 & NA & NA \\
\hline 4-chlomoaniline & 41 & 42 & $\varangle 2$ & 40 & $<0$ & NA & NA \\
\hline Hexachlordutadiere & $\triangleleft 1$ & 42 & 42 & 40 & $\triangleleft 0$ & NA & NA \\
\hline 4-Chloro-3-methylphemol & 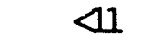 & $\triangle 2$ & $<2$ & $<0$ & $\triangleleft 0$ & NA & NA \\
\hline 2-Methylneqhthalene & $\triangleleft 1$ & 42 & $\varangle 2$ & 40 & $<0$ & NA & NA \\
\hline Hexachlonoc & $\triangleleft 1$ & $\varangle 2$ & $\varangle 2$ & $\triangleleft 0$ & $\triangleleft 0$ & NA & NA \\
\hline 2,4,6-Trichlorophenol & 41 & 42 & $\varangle 2$ & 40 & 40 & NA & NA \\
\hline 2,4,5-Trichlonquhenol & $\leqslant 7$ & $<8$ & $\varangle 60$ & $<0$ & $\diamond 0$ & NA & NA \\
\hline 2-Chlonaraphthalene & $<1$ & $\triangleleft 2$ & $\varangle 2$ & $<0$ & $<0$ & NA & NA \\
\hline 2-Nit & 57 & $<8$ & $\varangle 6$ & $\diamond 0$ & $\$ 0$ & NA & NA \\
\hline Dimethylphthalate & 41 & 42 & $\triangleleft 2$ & $\triangle 10$ & $<10$ & NA & NA \\
\hline Acenap & $\triangleleft 1$ & 42 & 42 & $\triangleleft 0$ & $<0$ & NA & NA \\
\hline 2,6-Dinitrotoluene & $\triangleleft 1$ & $\triangle 2$ & $\varangle 2$ & $<0$ & $\triangleleft 0$ & NA & NA \\
\hline 3-Nitroaniline & 57 & $<8$ & $\varangle 60$ & $<0$ & $\diamond 0$ & NA & NA \\
\hline Acenapl & 41 & $<2$ & $\varangle 2$ & $<0$ & $\triangleleft 0$ & NA & NA \\
\hline 2,4-Dinitrophemol & 57 & $<8$ & $<60$ & $<0$ & $<0$ & NA & NA \\
\hline 4-Nitro & 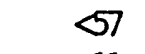 & $<8$ & $\varangle 60$ & $<0$ & $\diamond 0$ & NA & NA \\
\hline Diberzofuran & $\triangle 1$ & $\triangle 2$ & $\ll 2$ & $\triangleleft 0$ & $<0$ & NA & NA \\
\hline 2,4-Dinitrotoluene & 41 & 42 & $\varangle 2$ & $<0$ & $\triangleleft 0$ & NA & NA \\
\hline Diethylphthalate & $\triangleleft 1$ & $<2$ & $\varangle 2$ & 40 & $<0$ & NA & NA \\
\hline 4-Chlorophenyl-phenylether & $\triangle 1$ & $\triangleleft 2$ & $\varangle 2$ & $\triangleleft 0$ & 40 & NA & NA \\
\hline Fluovere & 41 & 42 & $\varangle 2$ & $<0$ & $\triangleleft 0$ & NA & NA \\
\hline 4-Nitroaniline & 57 & $<8$ & $\varangle 60$ & $<0$ & $<50$ & NA & NA \\
\hline 4,6-Dinitro-2-methylphenol & $<7$ & 58 & $\varangle 6$ & $<0$ & $<0$ & NA & NA \\
\hline N-nitrosodiphenylamine & 41 & $\varangle 2$ & $\varangle 2$ & $\triangle 0$ & $<0$ & $\mathrm{NA}$ & NA \\
\hline enylether & $\triangleleft 1$ & $\varangle 2$ & $\varangle 2$ & $<0$ & $<0$ & NA & NA \\
\hline Hexachlorob & 41 & $\varangle 2$ & $\varangle 12$ & 40 & $<10$ & NA & NA \\
\hline Pertachlorophenol & 87 & 58 & 860 & $\diamond 0$ & $<0$ & NA & $N A$ \\
\hline Fhenanthrene & $\triangleleft 1$ & $\varangle 2$ & $\varangle 2$ & $\triangle 0$ & $<10$ & NA & NA \\
\hline Anthracene & $\triangleleft 1$ & 42 & $\varangle 2$ & $<0$ & $\triangleleft 0$ & $\mathrm{NA}$ & $\mathrm{NA}$ \\
\hline Di-n-butylythalate & $\sqrt{5}$ & $\varangle 2$ & $\varangle 2$ & $\Delta 0$ & $<0$ & NA & NA \\
\hline Fluoranthene & 41 & $\triangleleft 2$ & $\varangle 2$ & 40 & $\varangle 0$ & $N A$ & $\mathrm{NA}$ \\
\hline Pyrene & 41 & 42 & $\varangle 2$ & 40 & $<10$ & NA & NA \\
\hline Butylberzylphthalate & 41 & $\triangleleft 2$ & 42 & 40 & $<0$ & NA & $\mathrm{NA}$ \\
\hline 3,3'-Dichlardbenzidene & $<3$ & 83 & $<4$ & $<0$ & $<0$ & NA & NA \\
\hline Berzo(a) anthraoene & $凶 1$ & $\varangle 2$ & $\varangle 2$ & $<10$ & $\triangleleft 0$ & NA & $\mathrm{NA}$ \\
\hline Arrysene & $\Delta 1$ & $\triangleleft 2$ & $\varangle 2$ & $<10$ & $<0$ & NA & NA \\
\hline bis(2-Ethylhexyl)phthalate & $\ll$ & $\varangle 2$ & $<2$ & 40 & $<0$ & NA & NA \\
\hline
\end{tabular}


Table D.7 (oontinued)

Sampling Date:

Burial Trench Number:

O6Ap189 O5Ap189 05Ap189 $1040189104 p 189 \quad 29 \mathrm{Mm} 8929 \mathrm{Mn} 89$

Species/Chemical:

Di-n-Octylyhthalate

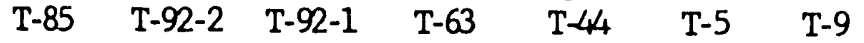

Berzo(b) fluoranthene

Berzo(k)fluoxanthene

Berzo(a)pyrene

Indern $(1,2,3-\infty$ pyrene

$\operatorname{Diberz}(a, h)$ antracene

Berzo(g,h,i)perylene

Acrylamide

Silver

Aluminum

Arsenic

Boran

Berium

Beryllitim

Calcium

Cadnium

Cobalt

Onranium

Copper

Inan

Merary

Potassium

Lithium

Magnesium

Menganese

Molybdenum

Soctium

Nickel

Hosphonus

Lead

Antimany

Selenium

Silioan

Tin

Strantium

Titanium

Vanadium

Zinc

Ziroanium

Branide

Chloride

Fluoride

Nitrate

Fhosphate

Sulfate

Total urganic Catban

(ppb) $\frac{\text { (ppb) }}{42}-(\mathrm{ppb})-(\mathrm{ppb})-(\mathrm{ppb})-(\mathrm{ppb})-(\mathrm{ppb})$

$\triangle 11 \quad \Delta 2 \quad \varangle 2 \quad 40 \quad 40 \quad N A \quad N$

$\triangle 11 \quad 42 \quad \varangle 2 \quad 40 \quad 40 \quad$ NA $\quad$ NA

$\triangle 1 \quad \varangle 2 \quad \varangle 2 \quad 40 \quad \varangle 0 \quad N A \quad N A$

$\triangle 11 \quad 42 \quad 42 \quad 40 \quad 40 \quad N A \quad N A$

$\triangle 1 \quad 42 \quad 42 \quad 40 \quad 40 \quad N A \quad N A$

$\begin{array}{lllll}41 & 42 \quad 42 \quad 40 \quad 40 \quad N A \quad N A\end{array}$

$\triangle 000 \quad \triangle 000 \quad \triangle 000 \quad \triangle 000 \quad \triangle 000 \quad N A \quad N A$

$\begin{array}{lllllll}9 & 8 & 5 & 12 & 13 & N A & \text { NA }\end{array}$

$\begin{array}{lllllll}50 & 50 & 50 & 340 & 50 & \mathrm{NA} & \mathrm{NA}\end{array}$

$\begin{array}{lllllll}50 & 50 & 50 & 67 & <0 & N A & N A\end{array}$

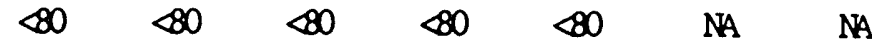

$\begin{array}{lllllll}280 & 150 & 150 & 200 & 290 & \text { NA } & \text { NA }\end{array}$

$\begin{array}{lllllll}\varangle .4 & \$ 0.4 & \$ .4 & \$ .4 & \$ .4 & \text { NA } & \text { NA }\end{array}$

$\begin{array}{lllllll}20000 & 48000 & 52000 & 140000 & 46000 & \mathrm{NA} & \mathrm{NA}\end{array}$

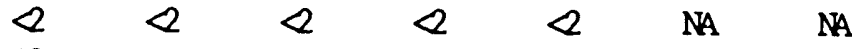

$\begin{array}{lllllll}43 & 7 & 33 & 9 & 26 & \text { NA } & \text { NA }\end{array}$

$\begin{array}{lllllll}5 & 5 & 3 & 13 & 10 & \text { NA } & \text { NA }\end{array}$

$\varangle 0 \quad 40 \quad 40 \quad 40 \quad 40 \quad N A \quad N A$

$8500 \quad 40 \quad 1100 \quad 40 \quad 19000 \quad$ NA $\quad$ NA

$\begin{array}{lllllll}0.5 & \varangle .1 & \varnothing .1 & 0.3 & 0.4 & \text { NA } & \text { NA }\end{array}$

$\begin{array}{lllllll}3010 & 2610 & 1800 & 3310 & 5210 & \text { NA } & \text { NA }\end{array}$

$\triangle 5000 \quad \varangle 5000 \quad 45000 \quad 45000 \quad 45000 \quad N A \quad N A$

$\begin{array}{lllllll}9600 & 7300 & 6800 & 16000 & 12000 & \text { NA } & \text { NA }\end{array}$

$\begin{array}{lllllll}36000 & 2600 & 270 & 4700 & 12000 & \text { NA } & \text { NA }\end{array}$

$\begin{array}{llllll}40 & 40 & 40 & 40 & 40 & N A\end{array}$

$\begin{array}{lllllll}3720 & 13100 & 10400 & 3780 & 5050 & \text { NA } & \text { NA }\end{array}$

$86 \quad 6 \quad 1100 \quad 66 \quad 65 \quad N A \quad N A$

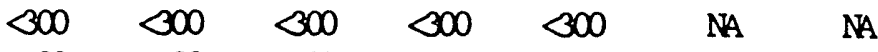

$\infty 0<30 \quad \infty 0 \quad 30 \quad \infty 0 \quad N A \quad N A$

$\$ 10 \quad 40 \quad \$ 0 \quad \varangle 0 \quad \$ 0 \quad N A \quad N A$

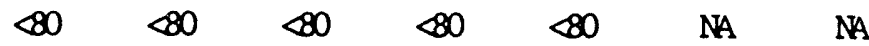

$\begin{array}{lllllll}4200 & 2300 & 2200 & 1300 & 1900 & \text { NA } & \text { NA }\end{array}$

$\begin{array}{llllll}50 & 50 & 50 & 50 & 50 & N A\end{array}$

$\begin{array}{lllllll}54 & 98 & 86 & 160 & 140 & \text { NA } & \text { NA }\end{array}$

$\infty 0 \quad 20 \quad 20 \quad<0 \quad 80 \quad N A \quad N A$

$44 \quad 4 \quad 4 \quad 4 \quad 4 \quad 4 \quad$ NA

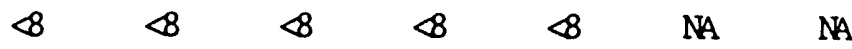

$\angle 0 \quad \angle 0 \quad \angle 0 \quad \angle 0 \quad \angle 0 \quad N A \quad N A$

$\begin{array}{lllllll}70 & <0 & <0 & 50 & 80 & N A & N A\end{array}$

$\begin{array}{lllllll}10500 & 7310 & 6750 & 4480 & 8810 & \text { NA } & \text { NA }\end{array}$

$\begin{array}{lllllll}2800 & 50 & 50 & 50 & 50 & \text { NA } & \text { NA }\end{array}$

$\begin{array}{lllllll}90 & 850 & 5040 & 2900 & 100 & \mathrm{NA} & \mathrm{NA}\end{array}$

$\begin{array}{lllllll}50 & 50 & 50 & 50 & 60 & N A & N A\end{array}$

$\begin{array}{lllllll}720 & 20400 & 17200 & 61900 & 1240 & \text { NA } & \text { NA }\end{array}$

$\begin{array}{lllllll}9400 & 1400 & 4800 & 4500 & 6000 & \text { NA } & \text { NA }\end{array}$ 
Table D.7 (continued)

\begin{tabular}{|c|c|c|c|c|c|c|c|}
\hline $\begin{array}{l}\text { Sampling Date: } \\
\text { Burial Trench Nuber: } \\
\text { Species/Chemical: }\end{array}$ & $\begin{array}{l}\text { O6Apl89 } \\
\text { T-85 } \\
\text { (ppb) } \\
\end{array}$ & $\begin{array}{r}05 \text { Ap189 } \\
\mathrm{T}-92-2 \\
\text { (ppb) } \\
\end{array}$ & $\begin{array}{r}05 A p 189 \\
\text { T- } 92-1 \\
- \text { (ppb) } \\
\end{array}$ & $\begin{array}{c}10 \mathrm{pp} 189 \\
\mathrm{~T}-63 \\
\text { (pd) } \\
\end{array}$ & $\begin{array}{c}10 \mathrm{pp} 189 \\
\mathrm{~T}-44 \\
\text { (ppb) } \\
\end{array}$ & $\begin{array}{c}29 \text { mar89 } \\
\mathrm{T}-5 \\
\text { (ppb) }\end{array}$ & $\begin{array}{l}29 \mathrm{Men} 89 \\
\mathrm{~T}-9 \\
(\mathrm{ppb})\end{array}$ \\
\hline & $(\mathrm{Bq} / \mathrm{L})$ & $(\mathrm{Bg} / \mathrm{L})$ & $(\mathrm{Bq} / \mathrm{L})$ & $(\mathrm{Bg} / \mathrm{L})$ & $(\mathrm{Bq} / \mathrm{L})$ & $\overline{(B g / L)}$ & $(\mathrm{Bg} / \mathrm{L})$ \\
\hline Canban-14 & 3 & 8 & 9 & $\infty 0$ & 9 & NA & NA \\
\hline Cobalt -60 & 8 & 8 & 2 & $<$ & $<$ & NA & NA \\
\hline Cesium-137 & 7.7 & 2.6 & 2 & 6.3 & 4.4 & NA & NA \\
\hline Gross Alpha & $\varangle .4$ & $\varangle .4$ & $\varangle .4$ & 0.20 & 0.44 & 7 & 2 \\
\hline Gross Beta & 14 & 7.5 & 5.4 & 13 & 5.1 & 512 & ND \\
\hline Strantium-90 & 3.3 & 0.88 & 2.3 & 2.0 & 1.0 & NA & NA \\
\hline Tritium & 860 & 880 & 930 & 99 & 970 & NA & NA \\
\hline Americium-241 & $\mathrm{NA}$ & NA & NA & NA & NA & NA & NA \\
\hline Arrium-244 & NA & NA & NA & NA & NA & NA & NA \\
\hline Plutanium-239 & $\mathrm{NA}$ & NA & NA & NA & NA & $\mathrm{NA}$ & NA \\
\hline Uranium-232 & NA & NA & NA & NA & NA & NA & NA \\
\hline Uranim-233 & NA & NA & NA & NA & NA & NA & NA \\
\hline $\mathrm{pH}$ & 7.65 & 7.65 & 7.7 & 7.9 & 7.8 & 8.1 & 6.1 \\
\hline Electrical Conductivity & 260 & 200 & 253 & 464 & 371 & 617 & 88 \\
\hline Dissolved Solids & 160 & 120 & 180 & 400 & 80 & 520 & 20 \\
\hline Total Solids & 1300 & 100 & 2180 & 500 & 220 & NA & NA \\
\hline Suspended Solids & 11140 & 0 & 2000 & 100 & 140 & NA & NA \\
\hline Handress & 126 & 134 & 146 & 366 & 172 & 332 & 16 \\
\hline Allkalinity & 17 & 134 & 129 & 322 & 272 & 276 & 21 \\
\hline
\end{tabular}


Thable D.8. Chamical analyses of SUSA 6 burial trench leachates

\begin{tabular}{|c|c|c|c|c|c|c|c|}
\hline $\begin{array}{l}\text { Sampling Date: } \\
\text { Burial Trench Nuber: } \\
\text { Species/Chamical: }\end{array}$ & $\begin{array}{c}29 \operatorname{ar} 89 \\
T-8 \\
(\mathrm{pdb}) \\
\end{array}$ & $\begin{array}{l}\text { 29Mar89 } \\
\text { T-2 } \\
\text { (ppb) }\end{array}$ & $\begin{array}{l}29 \text { Mar89 } \\
T-3 \\
\text { (ppb) }\end{array}$ & $\begin{array}{c}104 p 189 \\
T-57 \\
\text { (ppb) }\end{array}$ & $\begin{array}{c}10 \text { Apl89 } \\
\text { T-60 } \\
\text { (pab) }\end{array}$ & $\begin{array}{c}05 \mathrm{pp} 189 \\
\text { T-69 } \\
\text { (ppb) }\end{array}$ & $\begin{array}{r}05 \mathrm{Ap} 189 \\
\mathrm{~T}-110 \\
\text { (pdb) } \\
\end{array}$ \\
\hline Chloramethane & NA & NA & $\overrightarrow{N A}$ & $\overrightarrow{N A}$ & $\mathrm{NA}$ & $N A$ & $\mathrm{NA}$ \\
\hline Bramamethane & NA & NA & NA & NA & $\mathrm{NA}$ & NA & NA \\
\hline Vinyl chloride & NA & NA & $\mathrm{NA}$ & NA & $\mathrm{NA}$ & NA & NA \\
\hline Chlonoethane & NA & NA & $\mathrm{NA}$ & NA & NA & NA & NA \\
\hline Methylene chloride & $\mathrm{NA}$ & NA & $\mathrm{NA}$ & NA & $\mathrm{NA}$ & NA & NA \\
\hline Acetane & NA & NA & NA & NA & NA & NA & NA \\
\hline Canban disulfide & NA & NA & $N A$ & NA & $\mathrm{NA}$ & NA & NA \\
\hline 1,1-Dichlonoethere & NA & NA & NA & NA & NA & NA & NA \\
\hline 1,1-Dichloroethane & $\mathrm{NA}$ & NA & NA & NA & NA & NA & NA \\
\hline 1,2-Dichloroethene (Total) & NA & NA & NA & NA & NA & NA & NA \\
\hline Chlonofom & NA & NA & NA & NA & NA & NA & NA \\
\hline 1,2-Dichloroethane & NA & NA & NA & NA & $\mathrm{NA}$ & NA & NA \\
\hline 2-Butanane & NA & NA & NA & NA & NA & NA & NA \\
\hline 1,1,1-Trichlonoethane & NA & NA & NA & NA & $\mathrm{NA}$ & NA & NA \\
\hline Camban tetrachloride & $\mathrm{NA}$ & NA & NA & NA & $\mathrm{NA}$ & NA & NA \\
\hline Vinyl acetate & NA & NA & NA & NA & $\mathrm{NA}$ & NA & NA \\
\hline Bramotichloramethame & NA & NA & NA & NA & NA & NA & NA \\
\hline 1,2-Dichloropropare & NA & NA & NA & $N A$ & NA & NA & NA \\
\hline cis-1,3-Dichlonopropene & NA & NA & NA & NA & $\mathrm{NA}$ & NA & NA \\
\hline Trichlorothene & NA & NA & NA & NA & $\mathrm{NA}$ & NA & NA \\
\hline Dibramochloramethane & NA & NA & NA & NA & NA & NA & NA \\
\hline 1,1,2-Trichlorcethane & NA & NA & NA & NA & NA & NA & NA \\
\hline Berzene & NA & NA & NA & NA & NA & NA & NA \\
\hline trans-1,3-Dichlonopropene & NA & NA & NA & NA & $\mathrm{NA}$ & NA & NA \\
\hline Bramaform & NA & NA & NA & NA & $\mathrm{NA}$ & NA & NA \\
\hline 4-Methyl-2-pentanane & NA & NA & NA & NA & NA & NA & NA \\
\hline 2-Hexanane & $\mathrm{NA}$ & NA & $\mathrm{NA}$ & NA & NA & NA & MA \\
\hline Tetrachloroethene & $\mathrm{NA}$ & NA & NA & NA & NA & NA & NA \\
\hline 1,1,2,2-tetrachloroethane & NA & NA & NA & NA & NA & NA & NA \\
\hline Toluene & NA & NA & $N A$ & NA & NA & NA & NA \\
\hline Chlonoberzene & NA & NA & NA & NA & NA & NA & NA \\
\hline Ethylbenzene & NA & NA & NA & NA & NA & NA & NA \\
\hline Styreme & NA & NA & NA & NA & NA & NA & NA \\
\hline Xylene (Total) & NA & NA & NA & NA & NA & NA & NA \\
\hline Phenol & NA & NA & NA & NA & NA & NA & NA \\
\hline bis(2-Chlonoethyl)ether & $N A$ & NA & $N A$ & $N A$ & NA & $N A$ & $N A$ \\
\hline 2-Chlorophenol & NA & NA & NA & NA & $\mathrm{NA}$ & $N A$ & NA \\
\hline 1,3-Dichlondbenzene & $N A$ & NA & $\mathrm{NA}$ & $N A$ & $\mathrm{NA}$ & NA & NA \\
\hline 1,4-Dichlonoberzene & NA & NA & NA & NA & NA & NA & NA \\
\hline Berzyl alochol & NA & NA & NA & NA & NA & NA & NA \\
\hline 1,2-Dichlordberzene & $N A$ & NA & NA & NA & NA & NA & NA \\
\hline 2-Methylphanol & $N A$ & NA & NA & $N A$ & $\mathrm{NA}$ & NA & NA \\
\hline bis(2-Chloroisopropyl)ether & $N A$ & NA & $\mathrm{NA}$ & NA & NA & NA & NA \\
\hline 4-Methylphanol & NA & NA & NA & NA & NA & NA & NA \\
\hline N-nitroso-di-n-propylamine & NA & NA & NA & NA & $N A$ & NA & NA \\
\hline Hexachloroethane & NA & $\mathrm{NA}$ & $\mathrm{NA}$ & NA & NA & NA & NA \\
\hline
\end{tabular}


Table D.8 (ontenued)

\begin{tabular}{|c|c|c|c|c|c|c|c|}
\hline $\begin{array}{l}\text { Sampling Date: } \\
\text { Burial Trench Number: } \\
\text { Species/Chemical: }\end{array}$ & $\begin{array}{c}29 \mathrm{Mar} 89 \\
\mathrm{~T}-8 \\
\text { (ppb) } \\
\end{array}$ & $\begin{array}{l}29 \operatorname{sen} 89 \\
T-2 \\
(\mathrm{ppb})\end{array}$ & $\begin{array}{l}29 \mathrm{Mar} 89 \\
\mathrm{~T}-3 \\
-\mathrm{(ppb})\end{array}$ & $\begin{array}{c}10 \mathrm{pp} 189 \\
\mathrm{~T}-57 \\
\text { (ppb) }\end{array}$ & $\begin{array}{c}10 \mathrm{Ap} 189 \\
\mathrm{~T}-60 \\
\text { (ppb) }\end{array}$ & $\begin{array}{c}\text { 05Apl189 } \\
\text { T-69 } \\
\text { (ppb) }\end{array}$ & $\begin{array}{r}\text { 05Ap189 } \\
\text { T-110 } \\
\text { (pob) }\end{array}$ \\
\hline Nitroberzene & NA & $\mathrm{NA}$ & $\mathrm{NA}$ & $N A$ & $\mathrm{NA}$ & $\overline{N A}$ & $\mathrm{NA}$ \\
\hline Isophonane & NA & NA & $\mathrm{NA}$ & $\mathrm{NA}$ & NA & NA & NA \\
\hline 2-Nitropherol & NA & NA & NA & NA & NA & NA & Na \\
\hline 2,4-Dimethylphenal & NA & NA & NA & NA & NA & NA & NA \\
\hline Benzoic acid & NA & NA & NA & NA & NA & NA & NA \\
\hline bis(2-chlonoethaxy)methane & NA & NA & $\mathrm{NA}$ & NA & NA & NA & NA \\
\hline 2,4-Dichlonghemal & $\mathrm{NA}$ & NA & NA & NA & NA & NA & NA \\
\hline 1,2,4-Trichlomobenzene & NA & NA & NA & NA & $\mathrm{NA}$ & NA & NA \\
\hline Nephthalene & $\mathrm{NA}$ & NA & NA & NA & $\mathrm{NA}$ & $\mathrm{NA}$ & NA \\
\hline 4-chlonoaniline & $\mathrm{NA}$ & NA & NA & $\mathrm{NA}$ & NA & NA & NA \\
\hline Hexachlonobutadiene & $\mathrm{NA}$ & $\mathrm{NA}$ & NA & NA & NA & NA & NA \\
\hline 4-Chloro-3-methylphemol & NA & NA & NA & NA & $\mathrm{NA}$ & NA & NA \\
\hline 2-Methylnephthalene & NA & NA & NA & NA & MA & NA & NA \\
\hline Hexachlonocyclopentadiene & $N A$ & NA & NA & NA & $\mathrm{NA}$ & NA & NA \\
\hline 2,4,6-Trichloncphenol & NA & NA & NA & NA & $\mathrm{NA}$ & NA & NA \\
\hline 2,4,5-Trichlonophemol & NA & NA & NA & NA & NA & NA & NA \\
\hline 2-Chloranephthalene & NA & NA & NA & NA & NA & NA & NA \\
\hline 2-Nitroaniline & $\mathrm{NA}$ & NA & NA & NA & NA & NA & NA \\
\hline Dimethylphthalate & NA & NA & NA & NA & $\mathrm{NA}$ & $\mathrm{NA}$ & $\mathrm{NA}$ \\
\hline Abenaphthylene & NA & NA & NA & NA & NA & NA & NA \\
\hline 2,6-Dinitrotoluene & NA & NA & NA & NA & NA & $\mathrm{NA}$ & NA \\
\hline 3-Nitroaniline & NA & NA & NA & NA & NA & NA & NA \\
\hline Aoenaphthene & NA & NA & NA & NA & $\mathrm{NA}$ & NA & NA \\
\hline 2,4-Dinitrophemol & NA & NA & $\mathrm{NA}$ & NA & NA & NA & NA \\
\hline 4-Nitropherol & NA & NA & NA & NA & NA & NA & NA \\
\hline Diberzofuran & NA & $\mathrm{NA}$ & $\mathrm{NA}$ & $\mathrm{NA}$ & $\mathrm{NA}$ & NA & NA \\
\hline 2,4-Dinitrotoluene & NA & $N A$ & NA & $\mathrm{NA}$ & $\mathrm{NA}$ & NA & NA \\
\hline Diethylphthalate & NA & NA & NA & NA & NA & NA & NA \\
\hline 4-Chlorophenyl-phenylether & NA & NA & NA & NA & NA & NA & $N A$ \\
\hline Fluorene & NA & NA & NA & NA & NA & NA & NA \\
\hline 4-Nitroaniline & NA & $\mathrm{NA}$ & NA & NA & NA & NA & NA \\
\hline 4,6-Dinitro-2-methylphenol & NA & NA & NA & NA & $\mathrm{NA}$ & NA & NA \\
\hline N-nitrosodiphenylamine & NA & NA & NA & NA & NA & NA & NA \\
\hline 4-bromophenyl-phenylether & NA & $N A$ & NA & $\mathrm{NA}$ & NA & NA & NA \\
\hline Hexachlonoberzene & $\mathrm{NA}$ & $\mathrm{NA}$ & $\mathrm{NA}$ & $\mathrm{NA}$ & $\mathrm{NA}$ & NA & NA \\
\hline Pentachlorophemol & NA & NA & NA & NA & NA & NA & NA \\
\hline Fhenanthere & NA & $N A$ & Na & NA & NA & NA & NA \\
\hline Anthracene & NA & NA & NA & NA & NA & NA & NA \\
\hline Di-n-butylphthalate & NA & $\mathrm{NA}$ & NA & NA & NA & NA & NA \\
\hline Fluocanthene & NA & NA & NA & NA & $\mathrm{NA}$ & NA & NA \\
\hline Pyrene & $\mathrm{NA}$ & NA & NA & NA & NA & NA & $N A$ \\
\hline Butylbenzylphthalate & $\mathrm{NA}$ & $\mathrm{NA}$ & NA & $N A$ & NA & NA & NA \\
\hline 3,3'-Dichlorabenzidene & NA & NA & $\mathrm{NA}$ & NA & $\mathrm{NA}$ & $\mathrm{NA}$ & $\mathrm{NA}$ \\
\hline Berzo(a)anthracene & NA & NA & NA & NA & NA & NA & NA \\
\hline Orryseme & NA & $N A$ & NA & NA & NA & NA & $\mathrm{NA}$ \\
\hline bis(2-Ethylhexyl)phthalate & $\mathrm{NA}$ & NA & NA & NA & $\mathrm{NA}$ & $\mathrm{NA}$ & $\mathrm{NA}$ \\
\hline
\end{tabular}


Table D.8 (continued)

\begin{tabular}{|c|c|c|c|c|c|c|c|}
\hline $\begin{array}{l}\text { Sampling Date: } \\
\text { Burial Trench Nuber: } \\
\text { Species/Ohemical: }\end{array}$ & $\begin{array}{c}29 \mathrm{Mar} 89 \\
\mathrm{~T}-8 \\
(\mathrm{ppb}) \\
\end{array}$ & $\begin{array}{l}29 \mathrm{Mar} 89 \\
\mathrm{~T}-2 \\
\text { (ppb) }\end{array}$ & $\begin{array}{l}29 \mathrm{Mar} 89 \\
\mathrm{~T}-3 \\
\text { (ppb) }\end{array}$ & $\begin{array}{c}10 \mathrm{pp} 189 \\
\mathrm{~T}-57 \\
\text { (ppb) }\end{array}$ & $\begin{array}{c}10 \mathrm{Ap} 189 \\
\mathrm{~T}-60 \\
\text { (ppb) }\end{array}$ & $\begin{array}{c}\text { 05Ap189 } \\
\text { T-69 } \\
\text { (ppb) }\end{array}$ & $\begin{array}{r}05 \text { Ap189 } \\
\mathrm{T}-110 \\
\text { (ppb) } \\
\end{array}$ \\
\hline Di-n-Octylyhthalate & $\mathrm{NA}$ & NA & $\mathrm{NA}$ & $\mathrm{NA}$ & $\mathrm{NA}$ & $\mathrm{NA}$ & NA \\
\hline Berzo(b)flupranthere & $N A$ & NA & NA & NA & NA & NA & NA \\
\hline Benzo(k) fluoranthene & $N A$ & NA & NA & NA & NA & NA & NA \\
\hline Berzo(a)pyrene & $\mathrm{NA}$ & NA & NA & $N A$ & NA & NA & NA \\
\hline Indeno $(1,2,3-\infty)$ pyrene & $\mathrm{NA}$ & NA & $\mathrm{NA}$ & NA & NA & NA & NA \\
\hline Diberz $(a, h)$ anthracene & $N A$ & NA & $N A$ & NA & NA & NA & NA \\
\hline $\operatorname{Berzo}(g, h, i)$ perylene & $\mathrm{NA}$ & NA & $\mathrm{NA}$ & NA & NA & NA & NA \\
\hline Acrylamide & NA & NA & $\mathrm{NA}$ & $\mathrm{NA}$ & $N A$ & NA & NA \\
\hline Silver & $N A$ & NA & NA & NA & NA & NA & $\mathrm{NA}$ \\
\hline Aluminm & $N A$ & NA & $\mathrm{NA}$ & NA & NA & NA & NA \\
\hline Arsenic & $N A$ & NA & NA & NA & NA & NA & NA \\
\hline Boran & $N A$ & NA & NA & NA & NA & NA & NA \\
\hline Berium & NA & NA & NA & $\mathrm{NA}$ & $\mathrm{NA}$ & NA & NA \\
\hline Beryllium & $N A$ & NA & NA & NA & NA & NA & NA \\
\hline Calcium & $N A$ & NA & NA & $\mathrm{NA}$ & NA & NA & - $N A$ \\
\hline Cactrium & $N A$ & NA & $\mathrm{NA}$ & NA & NA & NA & $\mathrm{NA}$ \\
\hline Cobalt & NA & NA & NA & NA & NA & NA & NA \\
\hline Orranium & $N A$ & NA & NA & NA & M & NA & NA \\
\hline Copper & NA & NA & $\mathrm{NA}$ & $\mathrm{NA}$ & $\mathrm{NA}$ & NA & NA \\
\hline Iran & NA & $\mathrm{NA}$ & NA & NA & $\mathrm{NA}$ & NA & NA \\
\hline Merary & $\mathrm{NA}$ & NA & $\mathrm{NA}$ & NA & NA & NA & NA \\
\hline Btassium & $\mathrm{NA}$ & NA & NA & NA & NA & $N A$ & NA \\
\hline Lithium & NA & NA & $\mathrm{NA}$ & NA & $\mathrm{NA}$ & NA & NA \\
\hline Magnesium & NA & NA & NA & $\mathrm{NA}$ & $\mathrm{NA}$ & $\mathrm{NA}$ & NA \\
\hline Menganese & $\mathrm{NA}$ & NA & $N A$ & NA & NA & NA & NA \\
\hline Molybdenum & $N A$ & NA & $N A$ & $N A$ & $\mathrm{NA}$ & NA & NA \\
\hline Sodium & $N A$ & NA & $N A$ & NA & $\mathrm{NA}$ & NA & NA \\
\hline Nickel & $\mathrm{NA}$ & NA & $N A$ & NA & $\mathrm{NA}$ & NA & NA \\
\hline Phosphons & $\mathrm{NA}$ & NA & $N A$ & NA & $\mathrm{NA}$ & $\mathrm{NA}$ & NA \\
\hline Lead & $N A$ & NA & $N A$ & $\mathrm{NA}$ & $\mathrm{NA}$ & $\mathrm{NA}$ & NA \\
\hline Antimany & $N A$ & NA & $N A$ & NA & $\mathrm{NA}$ & NA & $\mathrm{NA}$ \\
\hline Selenium & NA & NA & $N A$ & NA & NA & NA & NA \\
\hline Silioan & $N A$ & $N A$ & $\mathrm{NA}$ & NA & $\mathrm{NA}$ & NA & NA \\
\hline Tin & NA & NA & NA & $\mathrm{NA}$ & $\mathrm{NA}$ & $\mathrm{NA}$ & NA \\
\hline Strantium & $N A$ & NA & $N A$ & NA & $\mathrm{NA}$ & $N A$ & NA \\
\hline Titanium & $N A$ & NA & $\mathrm{NA}$ & $N A$ & $\mathrm{NA}$ & $\mathrm{NA}$ & NA \\
\hline Vanadium & $N A$ & NA & $N A$ & $N A$ & $\mathrm{NA}$ & $\mathrm{NA}$ & $N A$ \\
\hline Zinc & $N A$ & $\mathrm{NA}$ & $N A$ & NA & $N A$ & NA & $N A$ \\
\hline Ziroanium & $N A$ & $\mathrm{NA}$ & $\mathrm{NA}$ & $N A$ & $\mathrm{NA}$ & NA & $N A$ \\
\hline Bramide & $\mathrm{NA}$ & $\mathrm{NA}$ & $N A$ & NA & $N A$ & $\mathrm{NA}$ & NA \\
\hline Chloride & NA & NA & NA & NA & $\mathrm{NA}$ & NA & $\mathrm{NA}$ \\
\hline Fluoride & $N A$ & $N A$ & $\mathrm{NA}$ & $N A$ & $N A$ & NA & NA \\
\hline Nitrate & NA & $\mathrm{NA}$ & $N A$ & NA & $N A$ & NA & NA \\
\hline Phosphate & NA & NA & $N A$ & $N A$ & $N A$ & NA & NA \\
\hline Sulfate & NA & $\mathrm{NA}$ & NA & NA & $N A$ & NA & NA \\
\hline Totai úganic Cauban & MA & NA & NA & $\mathrm{NA}$ & $\mathrm{NA}$ & NA & NA \\
\hline & & & D-32 & & & & \\
\hline
\end{tabular}


Table D.8 (continued)

\begin{tabular}{|c|c|c|c|c|c|c|c|}
\hline $\begin{array}{l}\text { Sampling Date: } \\
\text { Burial Trench Number: } \\
\text { Species/Chemical: }\end{array}$ & $\begin{array}{c}29 \mathrm{Mar} 89 \\
\mathrm{~T}-8 \\
\text { (ppb) } \\
\end{array}$ & $\begin{array}{l}29 \mathrm{man} 89 \\
\mathrm{~T}-2 \\
\text { (ppb) }\end{array}$ & $\begin{array}{l}29 \mathrm{Mar} 89 \\
\mathrm{~T}-3 \\
\text { (ppb) }\end{array}$ & $\begin{array}{c}10 \mathrm{Ap} 189 \\
\mathrm{~T}-57 \\
\text { (ppb) }\end{array}$ & $\begin{array}{c}10 \text { ppl } 189 \\
\text { T-60 } \\
\text { (pob) }\end{array}$ & $\begin{array}{l}\text { 05Apl89 } \\
\text { T-69 } \\
\text { (ppb) }\end{array}$ & $\begin{array}{r}05 \mathrm{Ap} 189 \\
\mathrm{~T}-110 \\
\text { (ppb) } \\
\end{array}$ \\
\hline & $(\mathrm{Bq} / \mathrm{L})$ & $(\mathrm{Bg} / \mathrm{L})$ & $(\mathrm{Bq} / \mathrm{L})$ & $(B q / L)$ & $(\mathrm{Bq} / \mathrm{L})$ & $\overrightarrow{(B q / L)}$ & $\overline{(B q / L)}$ \\
\hline Camban-14 & NA & NA & NA & NA & NÁ & NA & NA \\
\hline Cobalt -60 & NA & NA & NA & NA & NA & NA & NA \\
\hline Cesilum-137 & NL & NA & NA & NA & NA & NA & NA \\
\hline Gross Alpha & 320 & 10 & 10 & $\mathbf{N}$ & $\mathbf{N}$ & 25 & ND \\
\hline Gross Beta & 14275 & 103 & 457 & 13 & 15 & 237 & 103 \\
\hline Strantium-90 & NA & NA & NA & NA & NA & NA & NA \\
\hline Tritium & $\mathrm{NA}$ & 110 & 2810 & 6600 & 640 & 850000 & 3000 \\
\hline Americium-241 & $\mathbf{N}$ & NA & NA & NA & NA & NA & NA \\
\hline Arrium-244 & $\mathrm{NA}$ & NA & NA & NA & NA & NA & NA \\
\hline Plutanium-239 & $\mathrm{NA}$ & NA & NA & NA & NA & NA & NA \\
\hline Uranium-232 & $\mathrm{NA}$ & NA & NA & NA & NA & NA & NA \\
\hline Uranium-233 & $\mathbf{N A}$ & NA & NA & NA & NA & NA & NA \\
\hline $\mathrm{pH}$ & 8.1 & 7.6 & 7.9 & 7.95 & 7.7 & 8.1 & 7.8 \\
\hline Electrical Canductivity & 6490 & 247 & 477 & 420 & 459 & 478 & 416 \\
\hline Dissolved Solids & 6360 & 200 & 320 & 340 & 340 & 360 & 320 \\
\hline Total Solids & $\mathrm{NA}$ & 2860 & 2400 & 17020 & 580 & 1080 & 1980 \\
\hline Suspended Solids & $\mathrm{NA}$ & 2660 & 2080 & 16680 & 240 & 720 & 1660 \\
\hline Hardhess & 5220 & 186 & 336 & 318 & 330 & 356 & 244 \\
\hline Alkalinity & 6511 & 169 & 294 & 282 & 301 & 340 & 274 \\
\hline
\end{tabular}


Thble D.9. Chemical analyses of ShSA 6 burial trench leachates

\begin{tabular}{|c|c|c|c|c|c|c|c|}
\hline $\begin{array}{l}\text { Sampling Date: } \\
\text { Burial Trench Number: } \\
\text { Species/Chemical: }\end{array}$ & $\begin{array}{c}\text { O6Ap189 } \\
\text { T-225 } \\
\text { (ppb) } \\
\end{array}$ & $\begin{array}{c}\text { 05Apl89 } \\
\text { T-363 } \\
\text { (ppb) } \\
\end{array}$ & $\begin{array}{c}\text { O6Apl89 } \\
\text { T-367 } \\
\text { (ppb) }\end{array}$ & $\begin{array}{r}\text { O6Ap189 } \\
\text { T-395 } \\
\text { (pob) }\end{array}$ & $\begin{array}{r}06 \mathrm{Ap} 189 \\
\mathrm{~T}-414 \\
\text { (ppb) }\end{array}$ & $\begin{array}{r}\text { 06Ap189 } \\
\mathrm{T}-453 \\
\text { (ppb) }\end{array}$ & $\begin{array}{l}300 c t 87 \\
T-2 \\
\text { (ppb) }\end{array}$ \\
\hline Chloramethane & NA & NA & NA & NA & $\mathrm{NA}$ & NA & $\overline{N A}$ \\
\hline Bromomethane & NA & NA & NA & NA & NA & NA & NA \\
\hline Vinyl chloride & NA & $N A$ & NA & NA & NA & NA & NA \\
\hline Chloroethane & NA & NA & NA & NA & NA & NA & NA \\
\hline Methylene chloride & NA & NA & NA & NA & NA & NA & NA \\
\hline Acetane & $\mathrm{NA}$ & NA & NA & NA & NA & NA & NA \\
\hline Canban disulfide & NA & NA & $\mathrm{NA}$ & NA & NA & NA & $\mathrm{NA}$ \\
\hline 1,1-Dichloroethene & NA & NA & NA & NA & NA & NA & $\mathrm{NA}$ \\
\hline 1,1-Dichloroethane & NA & NA & NA & NA & NA & NA & $\mathrm{NA}$ \\
\hline 1,2-Dichloroethere (Total) & $\mathrm{NA}$ & NA & NA & NA & NA & NA & NA \\
\hline Chloroform & NA & $\mathrm{NA}$ & NA & NA & NA & NA & $\mathrm{NA}$ \\
\hline 1,2-Dichlonoethare & NA & NA & NA & NA & NA & NA & NA \\
\hline 2-Butanare & $\mathrm{NA}$ & NA & $N A$ & NA & $\mathrm{NA}$ & NA & $\mathrm{NA}$ \\
\hline 1,1,1-Trichloroethane & NA & NA & $\mathrm{NA}$ & NA & NA & NA & $\mathrm{NA}$ \\
\hline Canban tetrachloride & $\mathrm{NA}$ & NA & NA & $\mathrm{NA}$ & NA & NA & $\mathrm{NA}$ \\
\hline Vinyl acetate & $\mathrm{NA}$ & NA & NA & NA & $\mathrm{NA}$ & NA & NA \\
\hline Bramotichloramethame & NA & NA & NA & NA & NA & NA & NA \\
\hline 1,2-Dichloropropane & NA & NA & NA & NA & NA & NA & NA \\
\hline cis-1,3-Dichloropropene & $\mathrm{NA}$ & $N A$ & $\mathrm{NA}$ & NA & $\mathrm{NA}$ & NA & NA \\
\hline Trichlorothene & NA & $N A$ & NA & NA & NA & NA & NA \\
\hline Dibramochlaramethane & NA & NA & $\mathrm{NA}$ & NA & $\mathrm{NA}$ & NA & NA \\
\hline 1,1,2-Trichloroethane & $\mathrm{NA}$ & $N A$ & NA & NA & NA & NA & NA \\
\hline Berzene & NA & NA & NA & NA & $\mathrm{NA}$ & NA & NA \\
\hline trans-1,3-Dichlonopropene & NA & $N A$ & NA & NA & NA & NA & NA \\
\hline Bramform & NA & NA & NA & NA & NA & NA & NA \\
\hline 4-Methyl-2-pentamane & NA & NA & $N A$ & NA & NA & NA & NA \\
\hline 2-Hexanane & NA & NA & $N A$ & NA & NA & NA & NA \\
\hline Tetrachloroethene & $\mathrm{NA}$ & $N A$ & $N A$ & NA & NA & NA & NA \\
\hline 1,1,2,2-tetrachlanoethane & NA & $N A$ & NA & NA & NA & NA & NA \\
\hline Toluene & $\mathrm{NA}$ & NA & NA & NA & NA & NA & NA \\
\hline Chlondberzene & NA & NA & NA & $\mathrm{NA}$ & $\mathrm{NA}$ & NA & $\mathrm{NA}$ \\
\hline Ethylberzene & $\mathrm{NA}$ & NA & NA & NA & NA & NA & NA \\
\hline Styrene & $N A$ & NA & NA & NA & $\mathrm{NA}$ & NA & NA \\
\hline Xylene (Total) & $\mathrm{NA}$ & NA & $N A$ & NA & NA & NA & $\mathrm{NA}$ \\
\hline Ahenol & NA & NA & NA & NA & NA & NA & NA \\
\hline bis(2-Chlorøethyl)ether & $\mathrm{NA}$ & $N A$ & $\mathrm{NA}$ & $\mathrm{NA}$ & NA & NA & NA \\
\hline 2-Chlorophenol & $\mathrm{NA}$ & $N A$ & NA & NA & NA & NA & NA \\
\hline 1,3-Dichlonoberzene & $\mathrm{NA}$ & $\mathrm{NA}$ & NA & NA & NA & NA & $\mathrm{NA}$ \\
\hline 1,4-Dichlonoberzene & NA & $\mathrm{NA}$ & NA & $\mathrm{NA}$ & $\mathrm{NA}$ & NA & NA \\
\hline Berzyl alochol & NA & NA & NA & NA & $\mathrm{NA}$ & NA & $\mathrm{NA}$ \\
\hline 1,2-Dichlonoberzene & NA & NA & NA & NA & NA & NA & NA \\
\hline 2-Methylpheml & $\mathrm{NA}$ & $N A$ & $\mathrm{NA}$ & NA & NA & NA & NA \\
\hline bis(2-Chloroisqpropyl)ether & NA & NA & NA & NA & $\mathrm{NA}$ & NA & NA \\
\hline 4-Methylphenol & $\mathrm{NA}$ & NA & NA & NA & NA & NA & NA \\
\hline N-nitroso-di-n-propylamire & NA & $N A$ & $\mathrm{NA}$ & NA & NA & NA & NA \\
\hline Hexachloroethane & $\mathrm{NA}$ & $\mathrm{NA}$ & NA & $N A$ & NA & NA & NA \\
\hline
\end{tabular}


Table D.9 (continued)

\begin{tabular}{|c|c|c|c|c|c|c|c|}
\hline $\begin{array}{l}\text { Sampling Date: } \\
\text { Burial Trench Nuber: } \\
\text { Species/Chemical: }\end{array}$ & $\begin{array}{r}\text { 06Apl89 } \\
\text { T-225 } \\
\text { (ppb) } \\
\end{array}$ & $\begin{array}{l}\text { 05Apl89 } \\
\text { T-363 } \\
\text { (ppb) }\end{array}$ & $\begin{array}{c}\text { 06Ap189 } \\
\text { T-367 } \\
\text { (ppb) }\end{array}$ & $\begin{array}{r}\text { 06Ap189 } \\
\text { T-395 } \\
\text { (ppb) }\end{array}$ & $\begin{array}{r}\text { 06Ap189 } \\
\mathrm{T}-414 \\
\text { (ppb) } \\
\end{array}$ & $\begin{array}{r}\text { O6Ap189 } \\
T-453 \\
\text { (ppb) }\end{array}$ & $\begin{array}{c}3000+87 \\
\mathrm{~T}-2 \\
\text { (ppb) }\end{array}$ \\
\hline Nitrobenzene & $\mathrm{NA}$ & $\mathrm{NA}$ & $\mathrm{NA}$ & $\overline{\mathrm{NA}}$ & $\mathrm{NA}$ & $\mathrm{NA}$ & NA \\
\hline Isphorane & NA & NA & NA & NA & NA & $\mathrm{NA}$ & NA \\
\hline 2-Nitrophenol & NA & $\mathrm{NA}$ & $\mathrm{NA}$ & NA & NA & NA & NA \\
\hline 2,4-Dimethylphenol & NA & NA & NA & NA & NA & NA & NA \\
\hline Berzoic acid & NA & $\mathrm{Ni}$ & NA & NA & NA & NA & NA \\
\hline bis(2-chlonoethoxy)methane & NA & NA & NA & NA & NA & NA & NA \\
\hline 2,4-Dichlorophenol & NA & NA & $N A$ & NA & NA & NA & NA \\
\hline 1,2,4-Trichlordbenzene & $N A$ & NA & NA & $N A$ & NA & NA & $\mathrm{NA}$ \\
\hline Nephthalene & NA & NA & NA & NA & NA & NA & NA \\
\hline 4-chloroaniline & NA & $\mathrm{NA}$ & $N A$ & $\mathrm{NA}$ & $\mathrm{NA}$ & $\mathbb{N A}$ & $\mathrm{NA}$ \\
\hline Hexachlorobutadiene & MA & NA & NA & NA & NA & NA & NA \\
\hline 4-Chlono-3-methylphenol & NA & NA & NA & NA & NA & $\mathrm{NA}$ & $\mathrm{NA}$ \\
\hline 2-Methylnephthalene & NA & $\mathrm{NA}$ & $\mathrm{NA}$ & NA & NA & NA & $\mathrm{NA}$ \\
\hline Hexachlorocyclopentadiene & NA & NA & NA & NA & NA & NA & NA \\
\hline 2,4,6-Trichloropheml & NA & NA & NA & $\mathrm{NA}$ & NA & NA & $\mathrm{NA}$ \\
\hline 2,4,5-Trichlorophenol & NA & NA & NA & NA & NA & NA & NA \\
\hline 2-Chloranaphthalene & NA & NA & NA & NA & NA & NA & $\mathrm{NA}$ \\
\hline 2-Nitroaniline & NA & NA & NA & $\mathrm{NA}$ & NA & NA & NA \\
\hline Dimetinylythalate & NA & NA & $\mathrm{NA}$ & NA & NA & NA & NA \\
\hline Acenaphthylene & NA & NA & $\mathrm{NA}$ & NA & NA & NA & NA \\
\hline 2,6-Dinitrotoluene & NA & NA & NA & NA & $\mathbb{N A}$ & NA & NA \\
\hline 3-Nitroaniline & $\mathbb{N A}$ & NA & NA & iNA & NA & NA & NA \\
\hline Acenaphthene & $\mathrm{NA}$ & NA & $\mathrm{NA}$ & NA & NA & NA & NA \\
\hline 2,4-Dinitrophenol & $\mathrm{NA}$ & $N A$ & NA & NA & $\mathrm{NA}$ & $N$ & NA \\
\hline 4-Nitrophemol & NA & NA & $N A$ & NA & $\mathrm{NA}$ & NA & NA \\
\hline Diberzofuran & NA & NA & $N A$ & NA & NA & $\mathrm{NA}$ & NA \\
\hline 2,4-Dinitrotoluene & NA & NA & NA & NA & NA & NA & $\mathrm{NA}$ \\
\hline Diethylphthalate & $\mathrm{NA}$ & NA & NA & $\mathrm{NA}$ & NA & NA & $\mathrm{NA}$ \\
\hline 4-Chlorophenyl-phenylether & $\mathrm{NA}$ & $\mathrm{NA}$ & NA & NA & NA & NA & NA \\
\hline Fluorene & $\mathrm{NA}$ & $\mathrm{MA}$ & $\mathrm{NA}$ & A & NA & NA & NA \\
\hline 4-Nitroaniline & $\mathrm{NA}$ & NA & NA & NA & NA & NA & NA \\
\hline 4,6-Dinitro-2-methylyhenol & NA & NA & NA & NA & $\mathbb{N A}$ & NA & NA \\
\hline N-nitrosodiphenylamine & $\mathrm{NA}$ & NA & NA & NA & $\mathbb{N A}$ & NA & NA \\
\hline 4-bramopheryl-phenylether & NA & $N A$ & NA & $\mathrm{NA}$ & $\mathrm{MA}$ & NA & $\mathrm{NA}$ \\
\hline Hexachlonoberzene & NA & NA & NA & NA & NA & NA & NA \\
\hline Pentachloraphenol & $\mathrm{NA}$ & $\mathrm{NA}$ & NA & $\mathrm{NA}$ & NA & $\mathrm{NA}$ & NA \\
\hline Phenanthrere & $\mathrm{NA}$ & NA & NA & NA & NA & NA & NA \\
\hline Anthracene & $\mathrm{NA}$ & NA & $\mathrm{NA}$ & $\mathrm{NA}$ & $\mathrm{NA}$ & NA & NA \\
\hline Di-n-butylyhthalate & NA & NA & $\mathrm{NA}$ & NA & NA & $\mathrm{NA}$ & NA \\
\hline Fluoranthene & NA & NA & NA & NA & NA & NA & NA \\
\hline Pyzene & $\mathrm{NA}$ & $\mathrm{NA}$ & NA & NA & NA & NA & NA \\
\hline Butylberzylphthalate & Na & NA & NA & NA & NA & NA & NA \\
\hline 3,3'-Dichlomberzidene & NA & NA & NA & NA & NA & NA & NA \\
\hline Senzo(a)anthracene & NA & NA & NA & NA & NA & NA & NA \\
\hline 1. hrysene & NA & $N A$ & NA & NA & NA & Nì & NA \\
\hline bis(2-Ethylhexyl)phthalate & $N A$ & NA & $\mathbb{N A}$ & $\mathrm{NA}$ & NA & NA & $\mathrm{NA}$ \\
\hline
\end{tabular}


Table D.9 (continued)

\begin{tabular}{|c|c|c|c|c|c|c|c|}
\hline $\begin{array}{l}\text { Sampling Date: } \\
\text { Burial Trench Number: } \\
\text { Species/Chemical: }\end{array}$ & $\begin{array}{l}\text { O6Ap189 } \\
\text { T-225 } \\
\text { (ppb) } \\
\end{array}$ & $\begin{array}{c}\text { 05Ap189 } \\
\text { T-363 } \\
\text { (ppb) } \\
\end{array}$ & $\begin{array}{c}\text { O6Apl89 } \\
\text { T-367 } \\
\text { (ppb) }\end{array}$ & $\begin{array}{r}\text { O6Ap189 } \\
\text { T-395 } \\
\text { (ppb) }\end{array}$ & $\begin{array}{r}\text { O6Ap189 } \\
\mathrm{T}-414 \\
\text { (ppb) }\end{array}$ & $\begin{array}{r}\text { O6fpl89 } \\
\text { T-453 } \\
\text { (ppb) }\end{array}$ & $\begin{array}{l}300 \mathrm{ct87} \\
\mathrm{T}-2 \\
\text { (pob) }\end{array}$ \\
\hline Di-n-Octylphthalate & $\mathrm{NA}$ & NA & NA & $N A$ & $\mathrm{NA}$ & NA & $\mathrm{NA}$ \\
\hline Berzo(b)fluoranthene & NA & NA & $N A$ & NA & NA & NA & NA \\
\hline Berzo(k)fluoranthene & NA & NA & $N A$ & NA & NA & NA & NA \\
\hline Berzo(a)pyrene & NA & NA & $N A$ & NA & NA & NA & NA \\
\hline Indeno( $1,2,3-\infty d)$ pyrene & NA & NA & $N A$ & NA & NA & NA & $\mathrm{NA}$ \\
\hline Diberz $(a, h)$ anthracene & $\mathrm{NA}$ & NA & $N A$ & NA & NA & NA & $N A$ \\
\hline Benzo $(g, h, i)$ perylene & $\mathrm{NA}$ & NA & $N A$ & NA & NA & NA & NA \\
\hline Acrylamide & NA & NA & NA & NA & NA & NA & NA \\
\hline Silver & NA & NA & $N A$ & NA & NA & NA & NA \\
\hline Aluminum & NA & NA & $N A$ & NA & $\mathrm{NA}$ & NA & NA \\
\hline Arsenic & NA & NA & NA & NA & NA & NA & NA \\
\hline Bonan & $\mathrm{NA}$ & NA & NA & NA & NA & NA & NA \\
\hline Barium & NA & NA & $\mathrm{NA}$ & NA & $\mathrm{NA}$ & NA & NA \\
\hline Beryllium & $N A$ & NA & $N A$ & NA & NA & NA & NA \\
\hline Calcium & $N A$ & NA & $\mathrm{NA}$ & $N A$ & $N A$ & NA & NA \\
\hline Cadmium & $N A$ & $\mathrm{NA}$ & NA & NA & $\mathrm{NA}$ & NA & NA \\
\hline Cobalt & NA & NA & NA & $\mathrm{NA}$ & $\mathrm{NA}$ & NA & NA \\
\hline Chromium & $N A$ & NA & NA & NA & NA & NA & NA \\
\hline Copper & $N A$ & NA & NA & NA & $\mathrm{NA}$ & NA & NA \\
\hline Iran & $N A$ & NA & $N A$ & $\mathrm{NA}$ & $N A$ & NA & NA \\
\hline Merary & $N A$ & NA & $\mathrm{NA}$ & $N A$ & $\mathrm{NA}$ & $N A$ & NA \\
\hline Potassium & NA & NA & NA & NA & $\mathrm{NA}$ & NA & $\mathrm{NA}$ \\
\hline Lithium & $\mathrm{NA}$ & NA & $\mathrm{NA}$ & NA & $N A$ & $N A$ & NA \\
\hline Magnesium & NA & NA & $\mathrm{NA}$ & $N A$ & $N A$ & $N A$ & $\mathrm{NA}$ \\
\hline Manganese & NA & NA & $\mathrm{NA}$ & $N A$ & $N A$ & $N A$ & NA \\
\hline Mblybdenm & NA & MA & $\mathrm{NA}$ & $N A$ & $N A$ & $\mathrm{NA}$ & NA \\
\hline Sodium & $\mathrm{NA}$ & $N A$ & $N A$ & $N A$ & $N A$ & $\mathrm{NA}$ & $N A$ \\
\hline Nickel & NA & $N A$ & $\mathrm{NA}$ & $N A$ & $N A$ & $N A$ & NA \\
\hline Hosphonus & NA & NA & NA & NA & NA & NA & NA \\
\hline Lead & NA & NA & $N A$ & NA & NA & $N A$ & NA \\
\hline Antimany & NA & $\mathrm{NA}$ & $N A$ & NA & $\mathrm{NA}$ & $N A$ & $\mathrm{NA}$ \\
\hline Selenium & NA & NA & NA & $N A$ & $\mathrm{NA}$ & NA & NA \\
\hline Silioan & NA & NA & $\mathrm{NA}$ & $N A$ & NA & $N A$ & $\mathrm{NA}$ \\
\hline Tin & NA & $N A$ & $N A$ & $N A$ & $N A$ & NA & NA \\
\hline Strantium & NA & NA & $N A$ & NA & NA & NA & $N A$ \\
\hline Titanium & $N A$ & NA & $\mathrm{NA}$ & NA & $N A$ & $N A$ & NA \\
\hline Vanadium & $\mathrm{NA}$ & $\mathrm{NA}$ & NA & NA & $\mathrm{NA}$ & NA & NA \\
\hline Zinc & $N A$ & $N A$ & $N A$ & $N A$ & $N A$ & $N A$ & NA \\
\hline Ziroanium & $N A$ & $\mathrm{NA}$ & $N A$ & $N A$ & $N A$ & $N A$ & NA \\
\hline Branide & NA & $N A$ & $\mathrm{NA}$ & $N A$ & $\mathrm{NA}$ & $N A$ & NA \\
\hline Chloride & $N A$ & $\mathrm{NA}$ & $\mathrm{NA}$ & $N A$ & $N A$ & $N A$ & NA \\
\hline Fluoride & $\mathrm{NA}$ & $N A$ & NA & NA & $\mathrm{NA}$ & NA & $N A$ \\
\hline Nitrate & $\mathrm{NA}$ & $N A$ & NA & $N A$ & NA & NA & NA \\
\hline Phosphate & $\mathrm{NA}$ & NA & $N A$ & NA & $N A$ & NA & NA \\
\hline Silfate & ins & 详 & in & $\ddot{\mathrm{NA}}$ & $\ddot{N A}$ & NÁ & NA \\
\hline Total Organic Camban & $N A$ & $\mathrm{NA}$ & NA & NA & NA & $N A$ & NA \\
\hline
\end{tabular}


Table D.9 (continued)

\begin{tabular}{|c|c|c|c|c|c|c|c|}
\hline $\begin{array}{l}\text { Sampling Date: } \\
\text { Burial Trench Number: } \\
\text { Species/Chemical: }\end{array}$ & $\begin{array}{r}\text { OGApl89 } \\
\text { T-225 } \\
\text { (ppb) } \\
\end{array}$ & $\begin{array}{l}\text { O5Ap189 } \\
\mathrm{T}-363 \\
\text { (ppb) }\end{array}$ & $\begin{array}{c}\text { OfApl189 } \\
\text { T-367 } \\
\text { (ppb) }\end{array}$ & $\begin{array}{r}\text { 06Ap189 } \\
\text { T-395 } \\
\text { (ppb) }\end{array}$ & $\begin{array}{r}\text { O6Ap189 } \\
\mathrm{T}-414 \\
\text { (ppb) }\end{array}$ & $\begin{array}{r}06 \mathrm{pp} 189 \\
\mathrm{~T}-453 \\
(\mathrm{ppb}) \\
\end{array}$ & $\begin{array}{l}300 \mathrm{ct87} \\
\mathrm{T}-2 \\
(\mathrm{ppb})\end{array}$ \\
\hline Camban-14 & $(\mathrm{Bg} / \mathrm{L})$ & $(B q / L)$ & $(\mathrm{Bq} / \mathrm{L})$ & $(\mathrm{Bg} / \mathrm{L})$ & $(\mathrm{Bq} / \mathrm{L})$ & $(B q / L)$ & $(B q / L)$ \\
\hline Cobalt -60 & $\mathrm{NA}$ & NA & NA & NA & NA & NA & NA \\
\hline Cesium-137 & $\mathrm{NA}$ & $N A$ & NA & NA & $\mathrm{NA}$ & NA & NA \\
\hline Gross Alpha & $N A$ & NA & NA & NA & NA & NA & NA \\
\hline Gross Beta & $\mathbf{N}$ & 20 & 3 & 3 & 3 & 2 & 117 \\
\hline Strantium-90 & 23 & 880 & 8 & 25 & 5 & 88 & 200 \\
\hline Tritium & $\mathrm{NA}$ & NA & $\mathrm{NA}$ & NA & NA & NA & NA \\
\hline Americium-241 & 2900 & 1900 & 6200 & 270 & 130 & 2100 & $N A$ \\
\hline arium-244 & NA & NA & $\mathrm{NA}$ & NA & NA & NA & NA \\
\hline Plutanium-239 & NA & NA & NA & $\mathrm{NA}$ & $\mathrm{NA}$ & NA & NA \\
\hline Uranium-232 & NA & NA & NA & NA & NA & NA & $\mathrm{NA}$ \\
\hline Uranium-233 & NA & NA & $\mathrm{NA}$ & $\mathrm{NA}$ & NA & NA & $\mathrm{NA}$ \\
\hline $\mathrm{pH}$ & NA & NA & $\mathrm{NA}$ & NA & NA & NA & NA \\
\hline Electrical Conductivity & $\begin{array}{l}7.4 \\
613\end{array}$ & 7.8 & 7.8 & 8.2 & 7.5 & 8.55 & 6.2 \\
\hline Dissolved Solids & $\begin{array}{l}613 \\
740\end{array}$ & 252 & 55 & 440 & 226 & 8580 & 170 \\
\hline Total Solids & $\begin{array}{r}740 \\
18960\end{array}$ & 120 & 360 & 960 & 200 & 1360 & 60 \\
\hline Suspended Solids & $\begin{array}{l}18960 \\
18220\end{array}$ & 3660 & 3800 & 15760 & 26020 & 2780 & $\mathrm{NA}$ \\
\hline Hardhess & $\frac{18220}{305}$ & 3540 & 3440 & 14800 & 25820 & 1420 & NA \\
\hline Alkalinity & $\begin{array}{l}306 \\
219\end{array}$ & 136 & 208 & 604 & 154 & 300 & 86 \\
\hline & $\boldsymbol{\nabla}$ & $1 / 1$ & 234 & 1073 & 83 & 2844 & 54 \\
\hline
\end{tabular}


Table D.10. Chemical amalyses of SHSA 6 burial trench leachates

\begin{tabular}{|c|c|c|c|c|}
\hline $\begin{array}{l}\text { Sampling Date: } \\
\text { Burial Trench Number: } \\
\text { Species/Chemical: }\end{array}$ & $\begin{array}{c}270 c t 870 \\
T-3 \\
(\mathrm{pb}) \\
\end{array}$ & $\begin{array}{c}\text { Cosbub7 } \\
\mathrm{T}-4 \\
\text { (ppb) }\end{array}$ & $\begin{array}{l}270 \mathrm{ct} 87 \\
\mathrm{~T}-5 \\
\text { (ppb) }\end{array}$ & $\begin{array}{c}300 c t 87 \\
T-6 \\
\text { (ppb) }\end{array}$ \\
\hline Chloramethane & NA & NA & NA & NA \\
\hline Bromonethane & NA & $\mathrm{NA}$ & $\mathrm{NA}$ & NA \\
\hline Vinyl chloride & NA & NA & $N A$ & NA \\
\hline Chilonoethane & NA & NA & NA & NA \\
\hline Methylene chloride & $\mathrm{NA}$ & NA & NA & NA \\
\hline Acetane & $\mathrm{NA}$ & NA & $\mathbb{N A}$ & NA \\
\hline Canban disulfide & NA & NA & NA & NA \\
\hline 1,1-Dichlonothene & $\mathrm{NA}$ & NA & NA & NA \\
\hline 1,1-Dichlonoethane & NA & NA & $\mathrm{NA}$ & NA \\
\hline 1,2-Dichloroethene (Total) & NA & NA & NA & NA \\
\hline Chloroform & NA & NA & $\mathrm{NA}$ & $\mathrm{NA}$ \\
\hline 1,2-Dichlorcethane & NA & NA & NA & NA \\
\hline 2-Butanane & $\mathrm{NA}$ & NA & NA & NA \\
\hline 1,1,1-Trichloroethane & $\mathrm{NA}$ & NA & NA & NA \\
\hline Canban tetrachloride & NA & NA & NA & NA \\
\hline Viryl acetate & NA & $\mathrm{NA}$ & NA & NA \\
\hline Bromodichlomemethene & NA & NA & NA & NA \\
\hline 1,2-Dichloropropane & $\mathrm{NA}$ & NA & NA & NA \\
\hline cis-1,3-Dichlonopropene & MA & NA & NA & NA \\
\hline Trichloroethene & NA & $\mathrm{NA}$ & NA & NA \\
\hline Dibromochloramethane & NA & NA & $\mathrm{NA}$ & NA \\
\hline 1,1,2-Trichlonoethane & NA & NA & NA & NA \\
\hline Bervene & NA & NA & NA & NA \\
\hline trans-1,3-Dichloropropene & NA & NA & NA & NA \\
\hline Bramoform & NA & NA & NA & $\mathrm{NA}$ \\
\hline 4-Methyl-2-pentanane & NA & NA & NA & NA \\
\hline 2-Hexamane & NA & NA & $\mathrm{NA}$ & $\mathrm{NA}$ \\
\hline Tetrachloroethene & NA & $\mathrm{NA}$ & NA & NA \\
\hline 1,1,2,2-tetrachloroethane & $\mathbb{N A}$ & $\mathrm{NA}$ & NA & NA \\
\hline Toluene & NA & NA & NA & NA \\
\hline Chloroberzene & $\mathbb{N A}$ & NA & $\mathrm{NA}$ & NA \\
\hline Ethylberzene & NA & $\mathrm{NA}$ & NA & $\mathrm{NA}$ \\
\hline Styrene & NA & NA & $\mathrm{NA}$ & NA \\
\hline Xylene (Total) & $N A$ & NA & NA & NA \\
\hline thenol & NA & NA & NA & NA \\
\hline bis(2-Chloroethyl)ether & $\mathrm{NA}$ & NA & NA & $\mathrm{NA}$ \\
\hline 2-Chlonophenol & NA & NA & NA & NA \\
\hline 1,3-Dichilonobenzene & $\mathrm{NA}$ & $\mathrm{NA}$ & NA & $\mathrm{NA}$ \\
\hline 1,4-Dichlonoberzene & $\mathrm{NA}$ & $\mathrm{NA}$ & NA & Na \\
\hline Berzyl aloohol & NA & NA & NA & Na \\
\hline 1,2-Dichloroberzene & $\mathrm{NA}$ & NA & NA & $\mathrm{Na}$ \\
\hline 2-Methylphenol & NA & NA & NA & Na \\
\hline bis(2-chloroisopropyl)ether & NA & NA & $\mathbb{N A}$ & NA \\
\hline 4-Yetringlecol & NA & NA & NA & NA \\
\hline N-nitroso-di-n-propylamine & $\mathrm{NA}$ & NA & $\mathrm{NA}$ & $\mathrm{NA}$ \\
\hline Hexachlonoethane & NA & NA & NA & NA \\
\hline
\end{tabular}


Thble D.10 (ontinued)

\begin{tabular}{|c|c|c|c|c|}
\hline $\begin{array}{l}\text { Sampling Date: } \\
\text { Burial Tremch Nunber: } \\
\text { Species/Chamical: }\end{array}$ & $\begin{array}{c}270 c t 87 \\
T-3 \\
\text { (ppb) } \\
\end{array}$ & $\begin{array}{l}\text { C3Nbv87 } \\
\mathrm{T}-4 \\
\text { (ppb) }\end{array}$ & $\begin{array}{l}270 c t 87 \\
\mathrm{~T}-5 \\
\text { (ppb) }\end{array}$ & $\begin{array}{c}300<+87 \\
\mathrm{~T}-6 \\
\text { (ppb) }\end{array}$ \\
\hline Nitroberzene & $\mathrm{NA}$ & $\mathrm{NA}$ & $\overline{\mathrm{NA}}$ & $\mathrm{NA}$ \\
\hline Isophonane & $\mathrm{NA}$ & NA & NA & NA \\
\hline 2-Nitrophenol & NA & NA & NA & NA \\
\hline 2,4-Dimethylphenol & $\mathrm{NA}$ & NA & $\mathrm{NA}$ & NA \\
\hline Berzoic acid & $\mathrm{NA}$ & NA & $\mathrm{NA}$ & NA \\
\hline bis(2-chlonoethaxy)methane & NA & NA & $\mathrm{NA}$ & NA \\
\hline 2,4-Dichlorophenol & NA & NA & NA & NA \\
\hline 1,2,4-Trichlaraberzene & NA & NA & NA & NA \\
\hline Neyhthalene & NA & NA & $\mathrm{NA}$ & NA \\
\hline 4-chlonoaniline & NA & NA & NA & NA \\
\hline Hexachlonobutadiene & $\mathrm{NA}$ & NA & $\mathrm{NA}$ & NA \\
\hline 4-chloro-3-methylphenol & $\mathrm{NA}$ & NA & $\mathrm{NA}$ & $\mathrm{NA}$ \\
\hline 2-Methylnaphthalene & NA & $N A$ & $\mathrm{NA}$ & NA \\
\hline Hexachlorocyclopentadiene & $\mathrm{NA}$ & NA & $\mathrm{NA}$ & NA \\
\hline 2,4,6-Trichlorophenol & $\mathrm{NA}$ & $\mathrm{NA}$ & NA & NA \\
\hline 2,4,5-Trichlorophenol & $\mathrm{NA}$ & NA & NA & NA \\
\hline 2-Chloranephthalene & NA & NA & NA & NA \\
\hline 2-Nitroariline & $\mathrm{NA}$ & NA & NA & NA \\
\hline Dimethylphthalate & NA & NA & $\mathrm{NA}$ & NA \\
\hline Aoenaphthylene & $\mathrm{NA}$ & NA & $\mathrm{NA}$ & NA \\
\hline 2,6-Dinitrotoluene & $\mathrm{NA}$ & NA & $\mathrm{NA}$ & NA \\
\hline 3-Nitroeniline & NA & NA & NA & NA \\
\hline Acenaphthene & $\mathrm{NA}$ & $\mathrm{NA}$ & $\mathrm{NA}$ & NA \\
\hline 2,4-Dinitrophenol & NA & NA & NA & NA \\
\hline 4-Nitrophenol & $\mathrm{NA}$ & NA & $\mathrm{NA}$ & NA \\
\hline Diberzofiran & $\mathrm{NA}$ & $\mathrm{NA}$ & $\mathrm{NA}$ & NA \\
\hline 2,4-Dinitrotoluene & NA & NA & $\mathrm{NA}$ & NA \\
\hline Diethylphthalate & $\mathrm{NA}$ & $\mathrm{NA}$ & $\mathrm{NA}$ & NA \\
\hline 4-Chlorophenyl-phenylether & $\mathrm{NA}$ & NA & $\mathrm{NA}$ & NA \\
\hline Fluorere & NA & NA & $N A$ & NA \\
\hline 4-Nitroanuline & $\mathrm{NA}$ & NA & $\mathrm{NA}$ & NA \\
\hline 4,6-Dinitro-2-methylyhenol & NA & NA & NA & NA \\
\hline N-nitrosodiphenylamine & $\mathrm{NA}$ & NA & $\mathrm{NA}$ & NA \\
\hline 4-buamophenyl-pherylether & $\mathrm{NA}$ & NA & NA & NA \\
\hline Hexachilonoberzene & $\mathrm{NA}$ & NA & NA & NA \\
\hline Fentachlorophenol & NA & NA & NA & NA \\
\hline Fhenanthrene & NA & NA & NA & NA \\
\hline Anthracene & $\mathrm{NA}$ & NA & NA & NA \\
\hline Di-n-butylphthalate & $\mathrm{NA}$ & $N A$ & $\mathrm{NA}$ & NA \\
\hline Fluoranthene & NA & NA & $\mathrm{NA}$ & NA \\
\hline Pyrene & $\mathrm{NA}$ & $\mathrm{NA}$ & $\mathrm{NA}$ & NA \\
\hline Butylberzylphthalate & $\mathrm{NA}$ & $\mathrm{NA}$ & $\mathrm{NA}$ & NA \\
\hline 3,3' -Dichlonoberzidene & NA & NA & NA & NA \\
\hline Berzo(a)anthraoene & NA & NA & NA & NA \\
\hline Unrysene & 觔 & 药 & $\ddot{N}$ & iN \\
\hline bis(2-Ethylhexyl)phthalate & NA & $N A$ & NA & NA \\
\hline
\end{tabular}


Thable D.10 (continued)

\begin{tabular}{|c|c|c|c|c|}
\hline $\begin{array}{l}\text { Sampling Date: } \\
\text { Bucial Tremch Nunber: } \\
\text { Species/Chamical: }\end{array}$ & $\begin{array}{c}270 \mathrm{cts7} \\
\mathrm{T}-3 \\
\text { (ppb) } \\
\end{array}$ & $\begin{array}{l}\text { OSNbve8 } \\
\mathrm{T}-4 \\
\text { (ppb) }\end{array}$ & $\begin{array}{l}270 \mathrm{ct} 87 \\
\mathrm{~T}-5 \\
-(\mathrm{ppb})\end{array}$ & $\begin{array}{l}300 \mathrm{ct87} \\
\mathrm{T}-6 \\
\text { (ppb) }\end{array}$ \\
\hline Di-n-Octylphthalate & $\mathrm{NA}$ & NA & $N A$ & $\mathrm{NA}$ \\
\hline Berzo(b)fluoranthene & NA & NA & NA & NA \\
\hline Berzo(k) fluoranthene & NA & NA & NA & NA \\
\hline Berzo(a)pyrene & $\mathrm{NA}$ & NA & $\mathrm{NA}$ & NA \\
\hline Indemo $(1,2,3-\infty d)$ pyrene & $\mathrm{NA}$ & iA & NA & NA \\
\hline $\operatorname{Diberz}(\mathrm{a}, \mathrm{h})$ anthracene & NA & NA & $\mathrm{NA}$ & $N A$ \\
\hline $\operatorname{Berzo}(\mathrm{g}, \mathrm{h}, \mathrm{i})$ perylene & NA & NA & NA & NA \\
\hline Acrylamide & NA & NA & NA & NA \\
\hline Silver & NA & NA & NA & NA \\
\hline Aluminum & $\mathrm{NA}$ & $\mathrm{NA}$ & $\mathrm{NA}$ & NA \\
\hline Arsenic & NA & $\mathrm{NA}$ & NA & NA \\
\hline Boran & $\mathrm{NA}$ & NA & $\mathrm{NA}$ & NA \\
\hline Barium & $\mathrm{NA}$ & $N A$ & $\mathrm{NA}$ & NA \\
\hline Beryllium & NA & $\mathrm{NA}$ & $\mathrm{NA}$ & NA \\
\hline Calcium & $\mathrm{NA}$ & $\mathrm{NA}$ & $N A$ & NA \\
\hline Cadmium & NA & $N A$ & $N A$ & NA \\
\hline Cobalt & $\mathrm{NA}$ & NA & $\mathrm{NA}$ & NA \\
\hline Ohramium & NA & $\mathrm{NA}$ & $\mathrm{NA}$ & NA \\
\hline Copper & NA & NA & NA & NA \\
\hline Iran & NA & $\mathrm{NA}$ & NA & NA \\
\hline Merary & $\mathrm{NA}$ & NA & $\mathrm{NA}$ & NA \\
\hline Potassium & $\mathrm{NA}$ & NA & NA & NA \\
\hline Lithium & $\mathrm{NA}$ & NA & NA & NA \\
\hline Magnesium & $\mathrm{NA}$ & NA & NA & NA \\
\hline Manganese & NA & NA & $\mathrm{NA}$ & NA \\
\hline Molybdenum & NA & NA & NA & $\mathrm{NA}$ \\
\hline Soctium & $\mathrm{NA}$ & $\mathrm{NA}$ & NA & NA \\
\hline Nickel & $\mathrm{NA}$ & NA & $\mathrm{NA}$ & NA \\
\hline Hosphonus & $\mathbb{N A}$ & $\mathrm{NA}$ & NA & NA \\
\hline Lead & $\mathrm{NA}$ & $\mathrm{NA}$ & $\mathrm{NA}$ & $\mathrm{NA}$ \\
\hline Antimany & NA & NA & $N A$ & NA \\
\hline Selenium & NA & $N A$ & NA & NA \\
\hline Silioan & NA & NA & $N A$ & NA \\
\hline $\operatorname{Tin}$. & NA & $\mathrm{NA}$ & NA & NA \\
\hline Strantium & NA & $\mathrm{NA}$ & NA & $\mathrm{NA}$ \\
\hline Titanium & $\mathrm{NA}$ & $\mathrm{NA}$ & $\mathrm{NA}$ & $\mathrm{NA}$ \\
\hline Vanadium & $\mathrm{NA}$ & $\mathrm{NA}$ & $\mathrm{NA}$ & NA \\
\hline Zinc & $\mathrm{NA}$ & NA & $N A$ & NA \\
\hline Zirconium & $N A$ & NA & $\mathrm{NA}$ & NA \\
\hline Bramide & $\mathrm{NA}$ & $N A$ & $\mathrm{NA}$ & NA \\
\hline Chloride & NA & $\mathrm{NA}$ & $\mathrm{NA}$ & $\mathrm{NA}$ \\
\hline Fluoride & $\mathrm{NA}$ & NA & NA & NA \\
\hline Nitrate & $\mathbb{N A}$ & NA & NA & NA \\
\hline Hosphate & NA & $\mathrm{NA}$ & $\mathrm{NA}$ & $\mathrm{NA}$ \\
\hline Silfate & sin & Nin & in & in \\
\hline Total Organic Canban & $\mathrm{NA}$ & NA & $N A$ & NA \\
\hline
\end{tabular}


Thble D.10 (continued)

\begin{tabular}{|c|c|c|c|c|}
\hline \multirow[t]{2}{*}{$\begin{array}{l}\text { Sampling Date: } \\
\text { Burial Trench Number: } \\
\text { Species/Chemical: }\end{array}$} & $\begin{array}{l}270 c t 87 \\
T-3 \\
\text { (ppb) }\end{array}$ & $\begin{array}{l}\text { Oonovid } \\
\mathrm{T}-4 \\
\text { (ppb) }\end{array}$ & $\begin{array}{l}270 \mathrm{ct} 87 \\
\mathrm{~T}-5 \\
\text { (ppb) }\end{array}$ & $\begin{array}{c}300 c t 87 \\
T-6 \\
(\mathrm{ppb})\end{array}$ \\
\hline & $(\mathrm{Bq} / \mathrm{L})$ & $(\mathrm{Bq} / \mathrm{L})$ & $(\mathrm{Bg} / \mathrm{L})$ & $(\mathrm{Bq} / \mathrm{L})$ \\
\hline Canbon-14 & NA & NA & NA & NA \\
\hline Cobalt- 60 & NA & NA & NA & NA \\
\hline Cesium-137 & NA & NA & NA & NA \\
\hline Gross Alpha & ND & 50 & 3117 & ND \\
\hline Gross Beta & 1717 & 346736 & 717 & 4284 \\
\hline Strantium-90 & NA & $\mathrm{NA}$ & NA & NA \\
\hline Tritium & NA & NA & NA & NA \\
\hline Americium-241 & N & NA & $\mathbf{N}$ & $\mathbf{N}$ \\
\hline arrium-244 & NA & NA & NA & NA \\
\hline Plutanium-239 & NA & NA & NA & NA \\
\hline Uranium-232 & NA & NA & NA & NA \\
\hline Uranium-233 & NA & $\mathrm{NA}$ & NA & NA \\
\hline $\mathrm{pH}$ & 7.2 & 6.4 & 6.7 & 6.1 \\
\hline Electrical Canductivity & 760 & 1100 & 510 & 740 \\
\hline Dissolved Solids & 480 & 200 & 440 & 420 \\
\hline Total Solids & NA & NA & NA & NA \\
\hline Suspended Solids & NA & $\mathrm{NA}$ & NA & NA \\
\hline Handhess & 242 & 168 & 184 & 298 \\
\hline Alkalinity & 166 & 446 & 68 & 158 \\
\hline
\end{tabular}

Explanation of Data Qualifiers (prefixes):

$<$ This qualifier indicates that the compound was analyzed for but not detected. The sample lower quantitation limit is listed. Data values of this type were not used in computing averages or frequencies of ocarrence.

$\mathrm{J}$ Inticates that the quantitative value is estimated. Ustally this qualifier is used before a value which is below the lower quantitation limit (i.e., the compand was detected). Data values of this type were used in computing averages and frequencies of ocarrence.

B This qualifier appears for a campand which westected in both the sample and its associated blark. Data values of this type were used in computing averages and frequancies of positive findings.

E This qualifier indicates that the reported concentration of the compound exceded the upper calibration limit of the method and was estimated by extrapolation. Data values of this type were used in computing averages and frequencies of ocarrence.

NA Not analyzed. 


\section{DISTRIBUTION}

1. H. L. Adair

2-6. T. L. Ashwood

7. L. V. Asplund/N. W. Durfee

8. L. D. Bates

9. H. L. Boston

10. T. W. Burwinkle

11. R. B. Clapp

12. K. W. Cook

13. J. H. Cushman

14. N. H. Cutshall

15. M. P. Farrell

16. D. E. Fowler

17. C. W. Francis

18. S. B. Garland II

19. C. W. Gehrs

20. C. D. Goins

21. D. F. Hall

22. S. G. Hildebrand

23. L. D. Hyde

24. D. D. Huff

25. R. H. Ketelle

26. A. J. Kuhaida

27. A. P. Malinauskas

28. W. M. McMaster

29. L. E. McNeese

30. G. K. Moore
31. J. B. Murphy

32. C. E. Nix

33-34. P. T. Owen

35. D. E. Reichle

36. G. E. Rymer

37. T. F. Scanlan

38. P. A. Schrandt

39. D. S. Shriner

40. R. L. Siegrist

41. D. K. Solomon

42-46. B. P. Spalding

47. S. H. Stow

48. D. W. Swindle

49. J. R. Trabalka

50. S. D. Van Hoesen

51. R. I. Van Hook

52. L. D. Voorhees

53. J. S. Watson

54. R. K. White

55. D. S. Wickliff

56. Central Research Library

57-61. ER Document Management Center

62-69. ESD Library

70-71. Laboratory Records Department

72. ORNL Patent Section

73. ORNL Y-12 Technical Library

74. Office of Assistant Manager for Energy Research and Development, DOE Field Office, Oak Ridge, P.O. Box 2001, Oak Ridge, TN 37831-8600

75. P. H. Edmonds, Radian Corporation, 120 S. Jefferson Circle, Oak Ridge, TN 37830

76. J. F. Franklin, Bloedel Professor of Ecosystem Analysis, College of Forest Resources, University of Washington, Anderson Hall (AR-10), Seattle, WA 98195

77. G. M. Hornberger, Professor, Department of Environmental Sciences, University of Virginia, Charlottesville, VA 22903

78. G. Y. Jordy, Director, Office of Program Analysis, Office of Energy Research, ER-30, G-226, U.S. Department of Energy, Washington, DC 20545

79. J. R. Kannard, Program Manager, Bechtel National, Inc., P.O. Box 350, Oak Ridge Corporate Center, 151 Lafayette Drive, Oak Ridge, TN 37830

80-83. W. E. Murphie, Department of Energy, Office of Environmental Restoration, Eastern Area D\&D Branch, EM-423 (GTN), Washington, DC 20545

84. R. H. Olsen, Vice President for Research, University of Michigan, 6643 Medical Science Building II, Ann Arbor, MI 48109-0620

85. A. Patrinos, Acting Director, Environmental Sciences Division, Office of Health and Environmental Research, ER-74, U.S. Department of Energy, Washington, DC 20585

86-87. S. S. Perkins, DOE Field Office, Oak Ridge, Information Resource Center, 105 Broadway, Oak Ridge, TN 37830

88. S. P. Riddle, DOE Field Office, Oak Ridge, P.O. Box 2001, Oak Ridge, TN 37831-8541

89. R. C. Sleeman, DOE Field Office, Oak Ridge, P.O. Box 2001, Oak Ridge, TN 37831-8541 
90. F. J. Wobber, Ecological Research Division, Office of Health and Environmental Research, Office of Energy Research, ER-75, U.S. Department of Energy, Washington, DC 20545

91-92. Office of Scientific and Technical Information, P.O. Box 62, Oak Ridge, TN 37831 
$=$
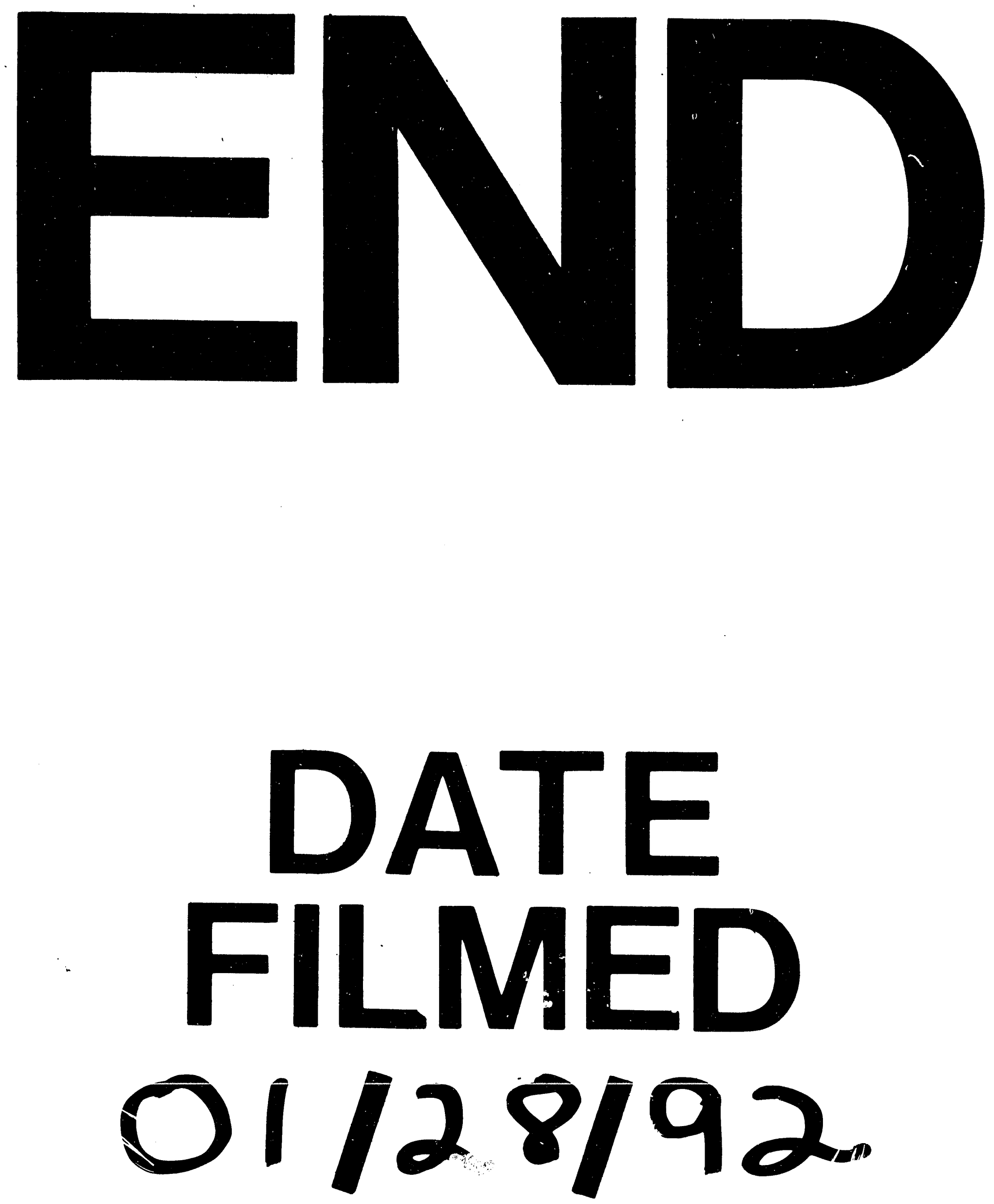
